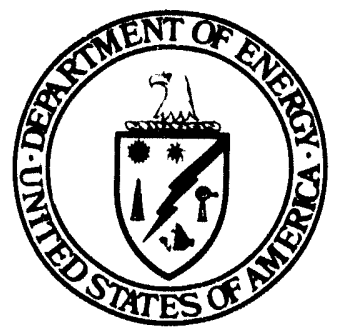

United States Department of Energy

Office of Energy Research

Office of Health and Environmental Research

Environmental Science Division

Washington, D.C. 20585

\title{
Global Change Research: Summaries of Research in FY 1993
}




\section{CONTENTS}

FOREWORD

PREFACE vii

INTRODUCTION

CLIMATE MODELING

Climate Research

Computer Hardware, Advanced Mathematics, and Model $\begin{array}{ll}\text { Physics (CHAMMP) Program } & 21\end{array}$

Program for Climate-Model Diagnosis and Intercomparison (PCMDI) 44

QUANTITATIVE LINKS 45

Quantitative Links Program 47

Atmospheric Radiation Measurement (ARM) Program 60

GLOBAL CARBON CYCLE 93

VEGETATION RESEARCH 105

OCEAN RESEARCH 119

ECONOMICS OF GLOBAL CLIMATE CHANGE

GLOBAL-CHANGE EDUCATION 145

INFORMATION AND INTEGRATION 149

NATIONAL INSTITUTE FOR GLOBAL ENVIRONMENTAL CHANGE 153

APPENDIX A: ADDRESSES OF PRINCIPAL INVESTIGATORS

APPENDIX B: ACRONYMS

SUBJECT INDEX

PRINCIPAL-INVESTIGATOR INDEX 211

INSTITUTION INDEX $\quad 215$ 
"Change" is everywhere these days.' From the change of administrations in Washington to the major geopolitical shifts on the international scene, we are experiencing profound changes that are affecting many aspects of our lives. The U.S. Global Change Research Program (USGCRP) is no exception, including the DOE component of the USGCRP.

Global change continues to be a very serious environmental concern with ever-increasing policy implications. The climate convention that was agreed upon in Rio de Janeiro, Brazil, last year is expected to be ratified very soon and is expected to stabilize the emissions of greenhouse gases of many countries, including the United States. Other global environmental issues, such as biodiversity, are receiving increasing attention and are expected to occupy a significant part of the scientific and political debate.

In response to the new national and intemational realities, the USGCRP is being reorganized to respond to the new challenges we face. The most significant restructuring involves a tighter coupling to the policy-making process within the federal government, exemplified by a significant initiative in the area of integrated (or "end-to-end") assessments. Other changes include an increased emphasis on impacts of global change, environmental biology, and activities dealing with mitigation and adaptation to global environmental change. Many of the changes will become evident in the administration's budget request for FY 1995, which will be presented to Congress early next year. However, we also expect to make significant adjustments to the FY 1994 program to prepare for a more orderly transition.

I am pleased to report that the USGCRP has finally put the Program Office in place with Mike
MacCracken as its Executive Director. Mike has taken a sabbatical from Lawrence Livermore National Laboratory to help provide a more scientific focus to the restructured USGCRP. Assisting him is a very capable cadre of program associates who are coordinating activities of the four working groups: long-term observations and data management, process research, integrated modeling, and assessments. DOE continues to chair the working group on process research and is providing staff for many activities of the Program Office. The Program Office is expected to better serve the USGCRP "customers" and will assist in meeting the U.S. commitments to the international scientific assessments.

Within the working group on process research we have worked hard this year to develop a research initiative on terrestrial ecosystems (TECO). The DOE entry to TECO is the Program for Ecological Research (PER), which has emerged from the restructuring of the DOE ecological-research programs. It is unclear how successful TECO will be in securing new resources, particularly among the land-management agencies, such as the departments of Interior and Agriculture. It is no secret that budgets will be very tight for the foreseeable future.

DOE will continue to emphasize the research to improve the prediction of climate change through such efforts as the Atmospheric Radiation Measurement (ARM) and the Computer Hardware, Advanced Mathematics, and Model Physics (CHAMMP) programs. Both programs are approaching maturity and will make valuable contributions to the integrated modeling activities of the USGCRP. We are also incorporating additional components of our division's research into the USGCRP in response to the restructuring guidance (e.g., the stratospheric and upper- 
tropospheric parts of our Atmospheric Chemistry Program and the Ocean Margins Program). In FY 1993, we launched our new initiative on economics related to climate change; this effort will grow significantly to include more direct support for integrated assessments in the context of a broader and coordinated interagency program.

It is important to mention that we have received funding from the Department of Defense (DOD) through the Strategic Environmental Research and Development Program (SERDP). SERDP funding is being applied to the development of instrumentation for unmanned aerospace vehicles (UAVs) and will support ARM and other components of the USGCRP. Because of the DOD funding, the effort is not described in this "Redbook." Interested readers may contact the ARM program manager for more information.

As we go to press, many more changes are under way. For example, the White House is restructur- ing the Federal Coordinating Council for Science, Engineering, and Technology (FCCSET) along with all its associated committees, such as the Committee on Earth and Environmental Sciences (CEES). New organizational arrangements will, no doubt, enhance the coordination of interagency activities, such as the USGCRP. The DOE Global Change Research Program is poised to serve in the new structure and to continue to provide solid scientific underpinning to research in this important environmental area.

The contributions of the program managers and the scientific investigators appearing in this volume are greatly appreciated as is the continued assistance of F. M. O'Hara, Jr., in its compilation and publication.

Ari Patrinos, Director

Environmental Sciences Division

Office of Health and Environmental Research 


\section{PREFACE}

This document describes the activities and products of the Global Change Research Program in FY 1993. The report is organized into four main sections.

SECTION

Introduction

Research Areas and

Project Descriptions

- Provides a mission statement, need, and mandate for a global-climate-change program

- Describes program relationships within the Department of Energy and the current situation regarding the globalclimate-change issue

- Lists the program objectives and specific activities

- Shows the distribution of research and the level of effort

- Describes scientific questions in each research area

- Provides descriptions of individual research projects

- Provides in-depth information on the research approach

- Lists the expected product for each project

- Shows the research approach or method used in each project

- Provides results to date when applicable

Appendixes

- Provide locator information on principal investigators for the overall report and define acronyms used in the text

Indexes

Provide indexes of keywords, principal investigators, and research institutions for easy reference

Questions concerning the Global Change Research Program or specific projects may be addressed to the Environmental Sciences Division, U.S. Department of Energy, ER-74, Washington, DC, 20585. The Program's telephone number is (301) 903-3281. 


\section{INTRODUCTION}

\section{MISSION STATEMENT AND NEED}

The Earth's climate is dependent on the radiative balance of the atmosphere, which in turn depends upon the input of solar radiation and the atmospheric abundances of radiatively active trace gases (i.e., greenhouse gases), clouds, and aerosols. The atmosphere naturally contains greenhouse gases that absorb and radiate back to Earth some of the longwave terrestrial radiation that would otherwise be radiated to outer space. This process adds to the net energy input to the lower atmosphere and the underlying surface, thereby increasing their temperatures and causing the Earth to be warmer than it otherwise would be.

Greenhouse gases result from both natural and manmade processes and include carbon dioxide $\left(\mathrm{CO}_{2}\right)$, nitrogen oxides $\left(\mathrm{N}_{\mathrm{x}} \mathrm{O}_{\mathrm{y}}\right)$, methane, chlorofluorocarbons, halogenated compounds, water vapor, and others. Since the industrial revolution, the atmospheric concentrations of several greenhouse gases have been increasing, primarily because of human activities. These increases have the potential to cause global climate change because of increased radiative forcing.

Global climate change is a significant issue for the Department of Energy (DOE) because energy production and use now contributes more than half of the total manmade emissions of greenhouse gases on a global basis. Energy policy affects the emissions of greenhouse gases because some energy sources emit more greenhouse gases than others and because that policy affects the mix of options for reducing emissions through reductions in energy demand and production. Conversely, changes in climate could affect energy systems and energy demand.
The mission of the Department's Global Change Research Program (GCRP) is (1) to predict the future atmospheric concentrations of $\mathrm{CO}_{2}$ and other energy-related greenhouse gases, (2) to predict the rate and magnitude of any potential climate change, (3) to understand and predict the impact of emissions and climate change on biota, and (4) to understand and predict the elemental economic costs and benefits of the potential impacts of global change and the options to mitigate the emission of energy-related greenhouse gases.

This information is required to understand from the scientific and economic perspectives the energypolicy options that are aimed at preventing, mitigating, or adapting to increasing greenhousegas concentrations and to global change. This Program will (1) elucidate the processes controlling the global carbon cycle and predict future levels of atmospheric $\mathrm{CO}_{2}$, (2) determine how changes in concentration of greenhouse gases may change the Earth's radiation balance, (3) acquire the data and develop the models to determine how changes in the Earth's radiation balance may change climate at global and regional scales and to predict rates of climate change, (4) develop the scientific methodology to determine and quantify the combined effect of climate change and greenhouse-gas concentrations on the biosphere, and (5) support the integrated economic assessments of global change. The Department's program is fully integrated with the USGCRP.

The GCRP will continue to support the DOE policy needs for scientific information and analyses on greenhouse gases, climate change, and biological effects related to global change. Principal foci will support the National Energy Policy Act of 1992 and the scientific contribution to international 
negotiations on climate. The GCRP will also provide DOE the scientific and fundamental economic tools to evaluate legislative proposals to combat global warming.

\section{MANDATES}

Global climate change is a national and international energy and environmental-science and public-policy issue. Energy-related emissions are considered the principal potential cause. DOE needs to pursue a vigorous research program to quantify the links between increased greenhouse gases, climate change, and biological effects. The national and international mandate clearly follows from the National Energy Policy Act (PL. 102486), the Intergovernmental Panel on Climate Change (IPCC) process, and the United Nations Conference on the Environment and Development (UNCED).

A long-term, interagency USGCRP, of which the GCRP is a component, is coordinated by the Federal Coordinating Council for Science, Engineering, and Technology's Committee on Earth and Environmental Sciences (CEES) and is Congressionally mandated by the Global Change Research Act of 1990 (PL. 101-606). The GCRP will continue to play an active role in the interagency USGCRP, and DOE will continue to press the CEES for an emphasis on research efforts that provide rapid closure on the global-climatechange issue.

\section{PROGRAM RELATIONSHIPS}

The goal of the GCRP is to provide scientific support for national and international policymaking concerning global change, with a primary focus on global climate change resulting from the increased levels of greenhouse gases produced by fossil-fuel combustion and other human activities. The research will contribute scientific information for DOE decisions about the environmental and economic acceptability of various energy technologies; research products are linked to environmental components of the National Energy Policy Act. The research also contributes directly to energy-policy concerns and evaluations linked to greenhouse gases, climate change, and biological effects of energy-induced global changes. Scientific information is provided to DOE's Environmental Issues Task Force - Global Climate Subgroup, which coordinates climate-related issues for DOE.

The GCRP cooperates with other CEES agencies and with international global-change research efforts to better leverage national and international scientific resources. Global-change research plans and products are integral parts of the U.S. Global Change Research Plan developed by the CEES. DOE leadership is exercised through the CEES, international bilateral agreements, and scientist-toscientist cooperation.

Spricific DOE research projects, such as the Atmospheric Radiation Measurement (ARM) Program and modeling programs, are linked to international activities of the World Climate Research Program; other activities, such as $\mathrm{CO}_{2}$ effects research and ocean $\mathrm{CO}_{2}$ measurements, are part of the International Geosphere-Biosphere Program. Global climate data is acquired through university grants, interagency research agreements, and international agreements, including agreements with the Chinese Academy of Sciences and the Commonwealth of Independent States.

The GCRP will continue its significant research contribution to U.S. Government-supported international activities, such as the Intergovernmental Panel on Climate Change and the United Nations Conference on Environment and Development, Agenda 21. A growing consensus is forming on the need for a collective international response to global climate change, and DOE's GCRP is providing basic scientific and economic support for assessments and negotiations.

\section{PROGRAM OBJECTIVES}

The major objectives of the Program are:

1. In Climate Modeling, to develop the tools necessary to predict accurately the global and regional climate change induced by increasing 
atmospheric concentrations of $\mathrm{CO}_{2}$ and other greenhouse gases. This component includes two subobjectives:

- To maintain core research on modeling of climate change, with attention to the coupled climate system of the atmosphere, ocean, biosphere, and cryosphere. A key element is support of the Program for Climate Model Diagnosis and Intercomparison [PCMDI, which includes the international general circulation model (GCM) intercomparison studies], whose work includes the study of clouds and the regional and interannual climate sensitivity to greenhouse gases. Climate-model development also improves the linkage of biosphere-climate and ocean-climate feedbacks for representing the energy and water fluxes of the land, atmosphere, and ocean.

- In the Computer Hardware, Advanced Mathematics and Model Physics (CHAMMP) Program, to accelerate and improve the prediction of the future response of global and regional climates to the increasing atmospheric concentrations of $\mathrm{CO}_{2}$ and other greenhouse gases. Developing an advanced cliniate model ( $\mathrm{ACM}$ ) will require the use of computers capable of increasing throughput by a factor of at least 10,000 , mathematical formulations and software that use the extensive parallelism of the emerging generation of computers, and improved algorithms that have been thoroughly verified with highly comprehensive sets of field observations.

2. In Quantitative Links, to join the predicted global and regional climate changes with observed changes in measurable Earth-system properties. Two subobjectives are identified:

- To maintain a core research program to develop the paleo, historical, and modem data sets essential for model verification and climatechange detection.

- In the ARM Program, to quantitatively describe the radiation balance from the surface to the top of the atmosphere and to determine the atmospheric characteristics responsible for this balance, to improve the parameterization of the formation and evolution of clouds in climate models, to create an experimental testbed for testing the process models used in GCMs and for the support of satellite-ground-truth measurements. The research involves a network of ground-based remote-sensing instruments along with campaign studies that use aircraft and tethered platforms. ARM focuses on quantitative links between greenhouse gases and climate change and examines climate feedbacks and energy fluxes in the coupled landatmosphere-ocean system. In addition, the research examines atmospheric cycling and transformation of radiatively and chemically important trace species.

3. In Global Carbon Cycle, to continue m'sasurement and modeling to estimate changes in the physical, chemical, and biological processes controlling sources and sinks of $\mathrm{CO}_{2}$. Measurement of $\mathrm{CO}_{2}$ fluxes among the atmosphere, biosphere, and land and ocean surfaces and of cycling of carbon within the terrestrial biosphere and the oceans is required for prediction of atmospheric $\mathrm{CO}_{2}$ change in relation to its release from the burning of fossil fuels and from deforestation.

4. In Vegetation Research, to focus increased attention on plant carbon metabolism and specifically to addresses basic biological mechanisms that affect direction, rate, and magnitude of $\mathrm{CO}_{2}$ fluxes and biotic responses to $\mathrm{CO}_{2}$ enrichment through DOE's unique research on plant responses to variable $\mathrm{CO}_{2}$ concentrations. The scope includes work on carbon dynamics of terrestrial, aquatic, and marine environments to quantify source and sink strengths of vegetation, soil, and sediments for carbon.

5. In Oceans Research, to conduct a global survey of $\mathrm{CO}_{2}$ in the ocean and to improve the oceancirculation models used for climate research. The research involves integrated laboratory, observational, and modeling studies to understand mixing, transport processes, and carbon cycling in the 
ocean and the exchange of heat and carbon between the ocean and the atmosphere. As part of the USGCRP, the DOE Ocean Research Program is focused on central questions of uncertainties about the rates of carbon and heat transport in the ocean and of changes in the oceanic $\mathrm{CO}_{2}$ reservoir. It is a critical component of the biogeochemical and ocean-dynamics elements of the USGCRP.

6. In Economics, to provide an integrated framework in which to compare the costs and benefits of different policy options that are intended to ameliorate potential impacts of climate change. This research includes investigation of the underlying economic forces that drive global change, including technology and innovation, and the tradeoffs that can be evaluated in an integrated framework, such as the comparative impact from the emission of various greenhouse gases.

7. In Global Change Education, to award competitive fellowships and scholarships at the postdoctoral, graduate, and undergraduate levels. Special emphasis will also be given to involving students in ongoing research at the national laboratories to achieve practical experience in the multidisciplinary sciences of global change.

8. In Information/Integration, to serve as a scientific interface through which technical information can be obtained, evaluated, qualityassured, and distributed; to promote and facilitate the exchange of data among scientists and between scientists and policymakers; and to perform highquality analyses of complex data to synthesize information needed in evaluating environmental issues.

\section{In the National-Institute of Global Environ-} mental Change (NIGEC), Congress created in 1989 an initiative to support DOE's GCRP primarily by providing funding for scientists in the academic community. Proposals submitted to NIGEC through the six regional centers are to address primarily the high-priority areas in the GCRP. The NIGEC goal for allocation of funding is: $75 \%$ for scientific aspects of global environmental change, $20 \%$ for research on policy options and strategies, and $5 \%$ on educational efforts. NIGEC is operated for DOE by the University of California under a cooperative agreement. It consists of six regional centers (the Northeastern Center at Harvard University, the Midwestern Center at Indiana University, the Southeastem Center at the University of Alabama, the Southcentral Center at Tulane University, the Western Center cohosted by the University of California at Davis and the University of California at Irvine, and the Great Plains Center at the University of Nebraska at Lincoln). Research supported through NIGEC includes projects that span a range of priorities in the USGCRP.

\section{DISTRIBUTION OF RESEARCH}

Because of the diverse types of research needed to meet the objectives of the research program, a wide range of research institutions and agencies is participating. DOE is supporting leading scientists in universities, at the national laboratories, in private industry, and in government agencies. The distribution of research funds to types of research institutions by fiscal year and for the overall program is presented in Fig. 1.

\section{LEVEL OF EFFORT}

Research sponsored by the GCRP has increased steadily since its beginning in FY 1978 when $\$ 1,479,000$ was made available for program development and research. By FY 1993, the budget reached more than $\$ 80,000,000$. The distribution of research funds to research areas by fiscal year and for the overall program is presented in Fig. 2.

\section{ORGANIZATION OF THE PROGRAM SUMMARY}

This publication describes all of the projects funded by the Environmental Sciences Division of DOE under annual contracts, grants, and interagency agreements in FY 1993. Each description contains the project's title; its 3-year funding history (in thousands of dollars); the period over which the funding applies; the name(s) of the prin- 


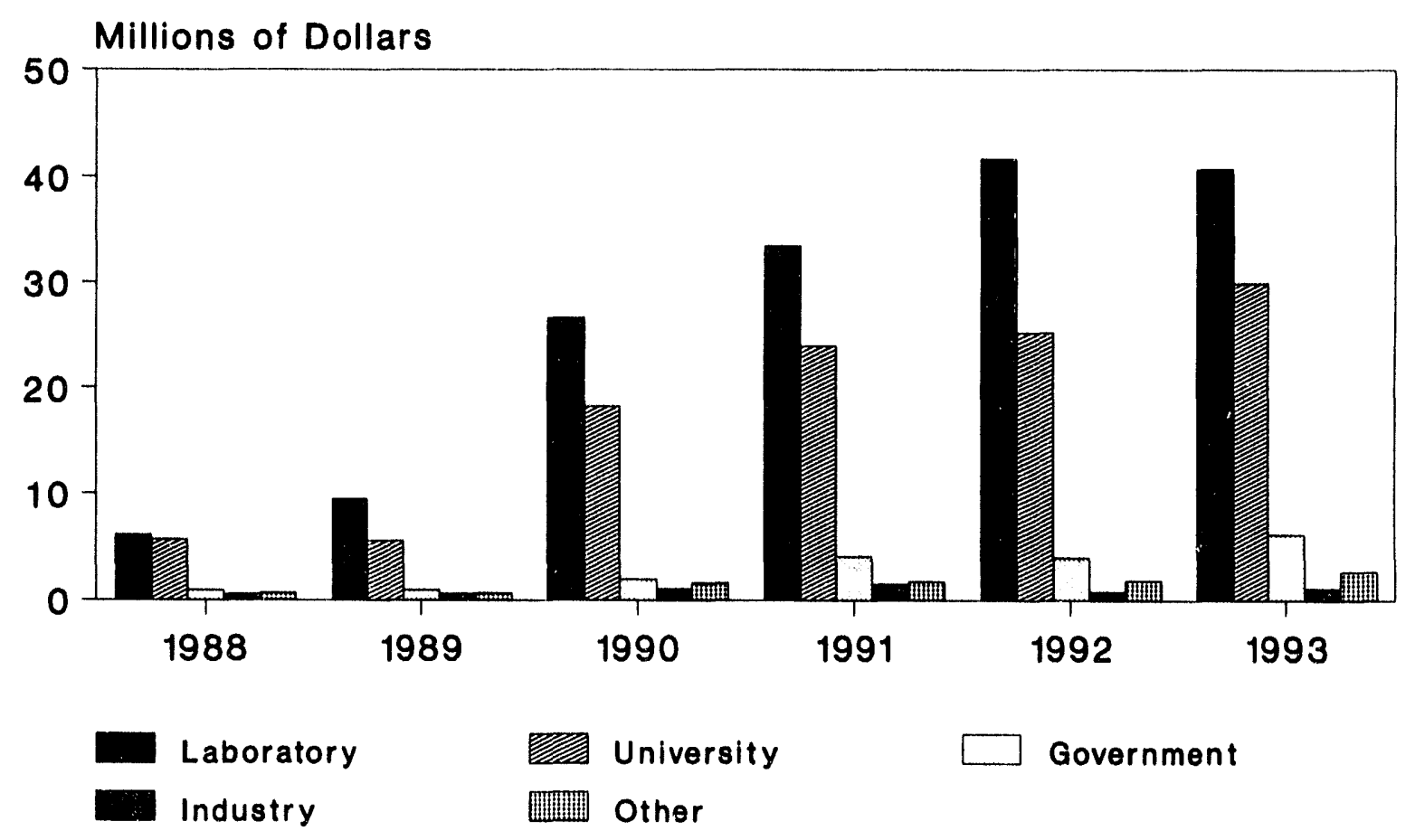

Fig. 1. Global-Change Budget History by Type of Institution.

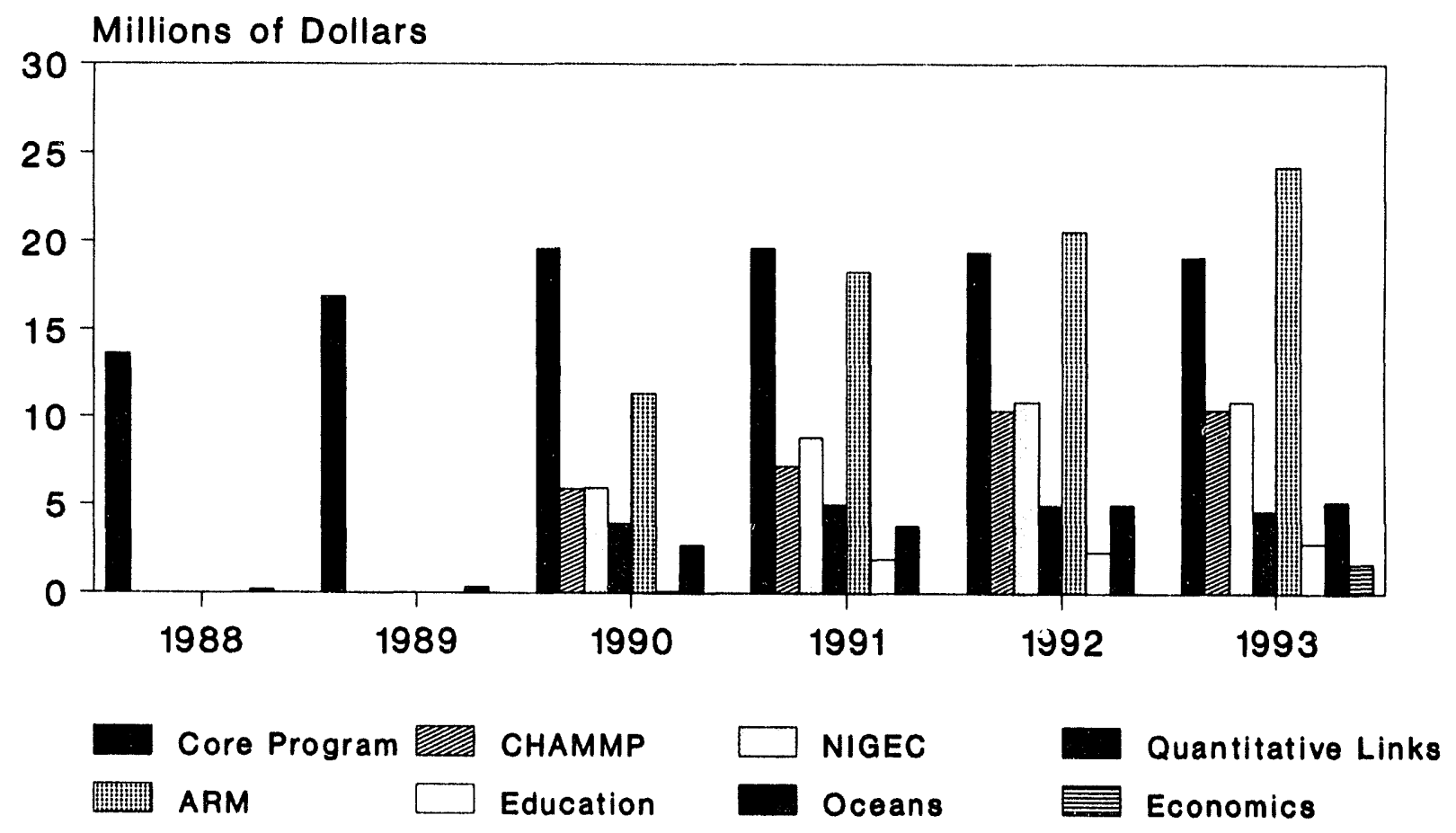

Fig. 2. Global-Change Budget History by Research Category. 
cipal investigator(s); the institution(s) conducting the projects; and the project's objectives, products, approach, and results to date (for most projects older than 1 year).

Project descriptions are categorized within the report according to program areas: climate modeling, quantitative links, global carbon cycle, vegetation research, ocean research, economics of global climate change, education, information and integration, and NIGEC. Within these categories, the descriptions are grouped alphabetically by principal investigator. Each program area is preceded by a brief text that defines the program area, states its goals and objectives, lists principal research questions, and identifies program managers.

\section{INDEXES AND KEYWORDS}

This document has been indexed to aid the reader in locating research topics, participants, and research institutions in the text and the project descriptions. Comprehensive subject, principal investigator, and institution indexes are provided at the end of the text for this purpose. The comprehensive subject index includes keywords from the Introduction and chapter texts in addition to those from the project descriptions. An appendix gives the addresses and telephone numbers of the participants in the main program. Another appendix lists and defines the acronyms used in this publication. 


\section{CLIMATE MODELING}

\section{Climate Research}

Climate defines the physical environment to which man and the biosphere respond. The principal components of the climate system are the atmosphere, the oceans, the cryosphere, and the land surface. As $\mathrm{CO}_{2}$ increases in the atmosphere, the climate will respond (e.g., atmospheric energy balance will be modified, with temperature expected to increase); ocean physical and chemical characteristics are also expected to change.

The many complex interactions within the climate system, called feedbacks, will further modify the response. For example, changes in cloudiness may either enhance or reduce a greenhouse warming. This is called cloud feedback. Another example is that the oceans can store large quantities of heat, possibly delaying evidence of the greenhouse effect. The general circulation models (GCMs) are currently incapable of realistically treating the clouds or oceans. Model improvement, data collection, and analysis will be required before the ocean-delay and cloud-feedback uncertainties can be resolved.

Specific information for integrated assessments is required to analyze changes that may impact people and the biosphere as the climate responds to increased concentrations of greenhouse gases. The primary information needs are:

- The rate of the global and regional climate change

- The distribution and magnitude of the global and regional climate change on a monthly basis

Current climate models cannot adequately provide the rate, distribution, and magritude of globai and regional climate change. To provide the needed information, why the models respond as they do must be understood, and the models must be developed further to provide the regional rate and magnitude of climate change.

Research Objectives. A main objective of the program is to develop and improve the capabilities to predict the range of global and regional climate change resulting from increasing $\mathrm{CO}_{2}$. This range includes, but is not limited to, the rate and magnitude of changes in temperature, precipitation, and frequency of extreme events, including changes in the variability of these quantities.

Another objective is to detect evidence of the climate response to the past and continuing increase in atmospheric $\mathrm{CO}_{2}$. This second objective addresses the question: Does the observed climate record reflect the changes that would be expected (as estimated from the climate-model projections), given the past and continued increases in atmospheric $\mathrm{CO}_{2}$ ?

A final objective is to provide the information needed for integrated assessments, such as regional changes in temperature, precipitation, soil moisture, and extreme events (which would be useful in analyses of food, fiber, and other resources) as well as information about direction, rate, and magnitude of regional climate change.

Research Questions. During the next 5 years of climate research, answers to the following questions will be developed:

- What are the physical and methodological (model-formulation) causes for the differences among models and between models and the observed climate? 
- Can GCMs, independently or in combination with other techniques, predict global and regional $\mathrm{CO}_{2}$-induced climate change? This effort will specify what information can be delivered (as requested by the other program areas) and when such estimates can be provided (e.g., estimate achievable spatial resolution and provide quantitative estimates of regional climate change with specific ranges of uncertainty).

- How rapidly can the climate change? To what extent will the ocean delay the climate change?

- Can a climate response to the greenhouse gases, particularly $\mathrm{CO}_{2}$, be observed? If not, can the time at which the signal might be expected to rise above the natural climate variability be estimated?

- What are the information requirements for resource analysis?

\section{Computer Hardware, Advanced Mathematics,} and Model Physics (CHAMMP)

Predicting the future climate response to the increasing atmospheric concentration of anthropogenic emissions will require climate models capable of much longer and more numerous simulations, finer resolution, and significantly better representation of the physics and chemistry of the climate system. Climate models with such capabilities will require computers capable of increasing throughput by a factor of at least $10^{4}$ over that obtainable in 1990, mathematical formulations and software that use the extensive parallelism of the emerging generations of computers, and improved algorithms that have been more thoroughly verified with more comprehensive sets of field observations.

The CHAMMP Program will develop coupled climate-system models employing the hardware and software capabilities offered $k$ " emerging computer architectures to address the challenging physics of understanding the climate system. The objectives of CHAMMP are phased over 10 years:
- Near Term (1990-1993): Achieve improved performance of existing climate-system models by taking advantage of emerging parallel-computing architectures

- Intermediate Term (1993-1995): Design and develop scaleable submodels of climate-system components that are capable of achieving more than 100 gigaflops (billion floating-point operations per second)

- Extended Term (1995-2000): Improve and assess the initial versions of the next-generation climate-system submodel components, assemble an optimized model, and execute scientifically valid simulations for long-term climate predictions at 10 teraflops (trillion floating-roint operations per second)

The CHAMMP initiative is designed to bring study of the climate system to the forefront of scientific problems, taking advantage of the computational capabilities that are being developed as part of the Federal High Performance Computing and Communications Program. Collaboration and coordination with these initiatives is an integral part of the CHAMMP initiative. Approximately one-quarter of computer resources at DOE's High Performance Computing Research Centers (HPCRCs) is devoted to the CHAMMP Program.

\section{Program for Climate Model Diagnosis and Intercomparison (PCMDI)}

The Program for Climate Model Diagnostics and Intercomparison (PCMDI) has as its principal mission the development of standards and tools for model diagnosis, validation, and intercomparison and the provision of assistance and collaboration with the climate-modeling community in comparing, documenting, and improving atmospheric (and eventually ocean and coupled atmosphereocean) models. The need for this activity has become increasingly apparent as more (and morecomplex) models are applied to climate-change problems. The level of disagreement among current models is significant, and to use these models for climate sensitivity and predictability studies in 
support of integrated assessments, the differences among models and between models and observations must be examined and accounted for in a systematic fashion.

As a contribution to more-comprehensive model diagnosis and intercomparison, PCMDI is coordinating the Working Group for Numerical Experimentation (WGNE) Atmospheric Model Intercomparison Project (AMIP). This activity calls for all current atmospheric models to simulate the observed atmospheric circulation with the monthly sea surface temperature and sea ice observed during the decade 1979-1988. By comparing the models' performance and through an exhaustive diagnosis and validation process, the systematic errors will be able to be isolated with confidence.

Other activities supported by PCMDI involve research into the issues of climate variability and predictability and examination of the problems of coupling. These activities offer the opportunity for further international cooperative research projects.

PCMDI is also supporting the intercomparison project Feedback Analysis for GCMs and Observations (FANGIO). The PCMDI role in this project is to assist with prototype simulations and analyses in an attempt to understand the differences in the models' calculated cloud radiative forcing. This activity has shown that clouds are one of the primary causes of the wide range of model responses to a prescribed sea-surface-temperature perturbation and has demonstrated the usefulness of multiple-model comparisons.

\section{Research Objectives}

- To contribute to our understanding of why different models produce different control climates and different responses to perturbations, including the analysis of feedback mechanisms.

- To improve the ability of models to simulate the climate of the recent past. If models are to be used to estimate the climatic effects of increas- ing $\mathrm{CO}_{2}$ and other greenhouse gases, model errors must be documented and all aspects of model performance must be compared with the best available observational data.

- To develop state-of-the-art diagnostic tools and techniques for use by the atmospheric-sciences community. The need for a suite of software tools available to the climate scientist at the workstation level has become increasingly apparent. Storage and access of model output is currently one of the major bottlenecks to effecive climate-model analysis.

Research Questions: During the next 5 years, PCMDI will address the following questions.

- What problems must be addressed to effectively couple atmospheric and oceanic GCMs, and how should intercomparison among coupled models bu carried out?

- Once the coupling is satisfactorily understood, what are the global and regional responses of the models to increasing greenhouse gases?

- What is the nature of the climate's natural (or unforced) variability on seasonal to centennial time scales, and what are the consequences for the predictability of climate change?

- How can we efficiently use several trillion bytes of model output and make meaningful diagnoses regarding the corresponding observed data?

\section{Program Managers}

Michael R. Riches and David C. Bader

Environmental Sciences Division

Department of Energy, ER-74

Washington, DC 20585

(301) 903-3264

Internet:

mike.riches@mailgw.er.doe.gov

dcb@dumbo.er.doe.gov 


\section{Research Summaries}

\section{Climate Research}

\author{
PARTIAL SUPPORT OF THE FY 1993 \\ COMMITTEE ON EARTH AND \\ ENVIRONMENTAL SCIENCES' WORKING \\ GROUP ON GLOBAL CHANGE (WGGC) \\ BAERWALD, THOMAS J. \\ COMMITTEE ON EARTH AND
ENVIRONMENTAL SCIENCES
}

$\begin{array}{lr}\text { FY } 1993 & 207 \\ \text { FY } 1992 & 0 \\ \text { FY } 1991 & 0\end{array}$

07/01/93-06/30/94

Objective: To provide partial support for activities supported by the Committee on Earth and Environmental Sciences (CEES) Subcommittee on Global Change Research (SGCR) and a provision for contingency.

Product: The efficient collection and distribution by a central body of legitimate and necessary costs for activties that are shared by agencies participating in the Global Change Research Program.

Approach: The National Science Foundation (NSF) will have the task of collecting funds for the CEES and distributing these funds for activities that are supported by the CEES as previously agreed to by all participating agencies:

- The National Academy of Sciences (NAS) Board on Global Change, which con hines the activities of five previous NAS boards and committees.

- The University Corporation for Aumospheric Research (UCAR) FY 1993 Summer Institutes Program.

- Printing costs for CEES WGGC documents.

- International activities.

- Staff, supplies, and equipment.
- CEES/SGCR Secretariat operating expenses.

- Education Working Group initiative.

\section{CLIMATE SYSTEM RESEARCH - STUDIES OF GLOBAL AND REGIONAL INSTRUMENT DATA}

BRADLEY, RAYMOND S.

UNIVERSITY OF MASSACHUSETTS
$\begin{array}{cc} & \\ \text { FY } 1993 & 227 \\ \text { FY } 1992 & 169 \\ \text { FY } 1991 & 185\end{array}$

$12 / 01 / 92-11 / 30 / 93$

Objective: To improve the climate data set and to develop global and regional analyses of the data to identify multiple indices of climate and climate change.

Product: Information for identifying climate change and for validating climate modeling results.

Approach: Circulation indices of secular climate variability will be developed by using the extensive sealevel-pressure data set to document the position of highand low-pressure systems, assess any changes in the center location, and subsequently infer atmospheric circulation. Understanding regional warming or cooling relative to global trends requires such collateral data. Although Southern Hemisphere data are sparse, data to compare the Northern and Southern Hemispheres for the past 40 years appears sufficient.

The temperature, precipitation, and other data necessary to characterize the $1920 \mathrm{~s}$, a period of rapid warming episodes, will be developed. Comparisons between the warming in the 1920 s and the 1980 s will be made to understand possible causal mechanisms.

In cooperation with other Division efforts and international studies, the DOE data set for regional temperature and precipitation changes will be analyzed. 
Seasonal and annual variability will be compared with decadal-scale events (e.g., El Niño) in an effort to document long-term natural variability.

Regional daily, monthly, and seasonal temperature and precipitation data sets will be analyzed to ascertain how the frequency of large events, which can dominate the monthly climate statistics of a region, differ in warm, cool, dry, and/or wet periods.

The record of long-term temperature variations will be extended for selected sites that have the potential to represent the hemispheric or global climate. The existing data and climate-model estimates of particularly sensitive areas will be $\mu$ sed to assist in the selection. Instrumental and proxy data will be used.

In cooperation with other grantees, the extent, quality, and utility of the DOE global climate data sets will be improved.

Results to Date: Different atmospheric-circulation indices have been examined to help interpret temperature and precipitation variations. These include Northern Hemisphere extratropical storm and anticyclone tracks, North Atlantic tropical-storm tracks, and wavenumber amplitudes of 500 -mb geopotential height for the Northern Hemisphere. Our analysis indicated that decadal-scale changes in regional precipitation are strongly related to anomalies in the position and strength of planetary-scale waves and that the most important wavenumbers are smaller than $\sim 5$.

Analysis of rapid warming episodes indicated that, although the 1980 s experienced a relatively large warming impulse compared to decadal changes in the previous century, other decades have had similar warming episodes, particularly when regional averages are considered.

Several additions to DOE data sets have been made, including records of storm tracks, daily maximum and minimum temperatures, and daily precipitation data. Updates of monthly mean temperature and precipitation for the existing archive of long-term stations is accomplished annually.

\section{RESEARCH PROJECT ON $\mathrm{CO}_{2}$-INDUCED CLIMATE CHANGE}

CESS, R. D., and HAMEED, S.

\section{STATE UNIVERSITY OF NEW YORK, STONY BROOK}

$\begin{array}{ll}\text { FY } 1993 & 449 \\ \text { FY } 1992 & 427 \\ \text { FY } 1991 & 419\end{array}$

$03 / 01 / 93-02 / 28 / 94$

Objective: To intercompare general circulation models (GCMs), documenting the physical processes responsible for agreement and disagreement and to statistically analyze regional climate data sets for relationships between large-scale and regional climates.

Product: An understanding of the GCMs' abilities to represent large-scale climate and the relationships between large-scale climate and regional climate.

Approach: More than 18 contemporary GCMs from throughout the world will be compared model-to-model and model-to-data. An experimental protocol will be developed for the model-to-model comparison with a $\pm 2^{\circ} \mathrm{C}$ sea-surface-temperature change as the forcing mechanism. The approach will assist in diagnosing cloud feedback and cloud forcing and the interactions of ice/snow albedo feedback and clouds as a probable cause for GCM disagreements. $\mathrm{CO}_{2}$ initial forcing and surface radiative balance will also be investigated.

The natural variability in climatic parameters will be studied. Subseasonal, annual, and interannual variations in air temperature, precipitation, and other climate parameters will be analyzed through observations and compared with their simulations in GCMs to quantitatively ascertain the deficiencies of the models and to demonstrate their regime of success: Climatic data developed by the National Climatic Data Center in the United States and the Institute of Geography of the Chinese Academy of Sciences will be used in this project.

Results to Date: The radiative forcing caused by a $\mathrm{CO}_{2}$ doubling has been compared for 15 GCMs. We found that these forcings differ substantially, ranging from 3.38 to $4.74 \mathrm{~W} / \mathrm{m}^{2}$ and that much of this variation is caused by differences in the models' radiation codes. 
We have shown that the simulated southern oscillation in the coupled ocean-atmosphere Oregon State University (OSU) GCM influences precipitation in Brazil and an adjacent area of the Atlantic with teleconnections that are similar to those found in observations. This investigation led to a method that identifies regions in the oceans where surface temperatures vary in unison with the precipitation on different time scales. Comprehensive frequency-domain analysis of sea-level-pressure (SLP) variations simulated by the OSU GCM showed that the frequency structure of the variability can be explained as summation and difference tones caused by the interaction of the annual cycle and its harmonics with the three subharmonic frequencies at the quasibiennial oscillation (QBO), the quasitriennial oscillation (QTO), and the atmospheric pole tide.

\section{ACCELERATED LINE-BY-LINE CALCULATIONS FOR THE RADIATIVE TRANSFER OF TRACE GASES RELATED TO CLIMATE STUDIES}

CLOUGH, SHEPARD A.

\section{ATMOSPHERIC AND ENVIRONMENTAL RESEARCH, INC.}

$\begin{array}{lr}\text { FY } 1993 & 68 \\ \text { FY } 1992 & 0 \\ \text { FY } 1991 & 0\end{array}$

$$
01 / 15 / 93-01 / 14 / 94
$$

Objective: To provide detailed, line-by-line (LBL) radiative codes to quantitatively study spectral outgoing radiances and to extend detailed LBL codes to the visible and ultraviolet spectral regions.

Product: Detailed information to test the parameterizations used in climate models.

Approach: Model calculations with a detailed LBL code indicate that during the past decade stratospheric ozone depletion could have radiatively offset the CFC greenhouse effect. The detailed calculations will support the testing of that hypothesis and help define data needs. A key result will be the spectral information that can be obtained from the LBL code.

How atmospheric cooling rates, at changing pressure levels, depend on spectral intervals will be investigated. The LBL code will be used to identify measurements possible for satellite systems and key spectral characteristics that GCMs must simulate.

The spectral region related to the net forcing of ozone and the validation of the shortwave model used in 2-D chemistry studies will be examined to understand the solar source function and the importance of molecular and particle scattering.

Results to Date: New project.

\section{THE IMPACT OF RECENT MODEL IMPROVEMENTS ON GISS GCM PREDICTIONS OF CLIMATE CHANGE}

\author{
DRUYAN, LEONARD M. \\ COLUMBIA UNIVERSITY

$\begin{array}{lr}\text { FY } 1993 & 54 \\ \text { FY } 1992 & 52 \\ \text { FY } 1991 & 0\end{array}$

$09 / 15 / 93-9 / 14 / 94$

Objective: To incorporate three new parameterizations into the NASA Goddard Institute for Space Studies (GISS) GCM and to test them by evaluating simulations forced by globally observed sea-surface temperatures.

Product: A significant advance in the GISS GCM being used in the DOE climate-model intercomparison and diagnosis program.

Approach: Three new parameterizations for the GISS GCM will be developed and tested. The moistcorivestion parameterization will include the downdrafts at convective seale to prevent the model's tendency to be too dry. Upper humidity and temperature profiles will be improved by using a two-part convective scheme for entraining and nonentraining convective plumes.

The grcund-hydrology parameterization will include multiple soil layers, hydraulic conductivity, soil texture, surface and subsurface transport, and ev apotranspiration.

Planetary boundary layer ( $\mathrm{PBL}$ ) parameterization will be improved by incorporating eddy transfer coefficients that better match observations. The improvements should improve convective precipitation and surface-wind and surface-temperature calculations. 
Each of these parameterizations will be tested separately and together at three model horizontal resolutions of $8^{\circ}$ $\times 10^{\circ}, 4^{\circ} \times 5^{\circ}$, and $2^{\circ} \times 2.5^{\circ}$. The $8^{\circ} \times 10^{\circ}$ resolution will be uses to tie back to the original model formulation. Tests will be run with the boundary conditions for the 10-year Atmospheric Model Intercomparison Program (AMIP), as appropriate.

Results to Date: Five-year simulations at $4^{\circ} \times 5^{\circ}$ horizontal resolution were made for the basic GCM as well as for alternative versions, each including a change in one of the model's component parameterizations. These runs used climatological sea-surface temperatures (SSTs). January and July mean fields for each version were compared with each other and with climatology. The most noticeable impacts were improvements in tropical surface winds attributable to the new formulation of the PBL.

Simulations of the contrasting 1987 and 1988 JuneAugust (JJA) seasons were found to be a suitable vehicle for making model intercomparisons, consistent with the recommendations of the Monsoon Experimentation Group of the World Meteorological Organisation's (WMO) Tropical Ocean Global Atmosphere (TOGA) project. The performance of the basic GCM for simulations forced by JJA 1987 and 1988 SST was thoroughly documented, highlighting several deficiencies in this model's tropical circulation. The evaluation was aided by the development of computer graphics that depict seasonal means of the $200-\mathrm{mb}$ velocity potential and stream function as diagnostic tools. Recent simulations based on a model version incorporating both the new PBL and a new moist-convection scheme show improvements, especially in the representation of 1988 vs 1987 differences in the Pacific Walker circulation and the Asian/African monsoon.

\section{ROLE OF LARGE-SCALE, EXTRATROPICAL DYNAMICS IN CLIMATE CHANGE: A STANSTEAD SEMINAR}

GUTOWSKI, WILLLAM J., JR.

IOWA STATE UNIVERSITY

$\begin{array}{lr}\text { FY 1993 } & 10 \\ \text { FY 1992 } & 0 \\ \text { FY 1991 } & 0\end{array}$

$04 / 01 / 93-03 / 31 / 94$
Objective: To support a multiagency workshop on the quantification of large-scale atmospheric structure and its role in climate predictability.

Product: An expert review of large-scale atmospheric structure and its role in climate predictability.

Approach: A multiagency-sponsored workshop will be conducted. The Stanstead Seminar will identify and discuss the role of large-scale extratropical systems on long-term climate dynamics. The objectives of the CHAMMP Program include the determination of factors that control the theoretical limits to climate prediction. The workshop will address the quantification of largescale atmospheric structure and its role in climate predictability. CHAMMP will provide approximately one-third of the funding for the seminar, with equal amounts contributed by NSF and NOAA.

Results to Date: New project.

\section{SOUTHERN OCEAN CONTROLS ON CURRENT ICE-SHELF EVOLUTION}

HELLMER, H. H., and JACOBS, S. S.

\section{COLUMBIA UNIVERSITY}

$\begin{array}{lr}\text { FY } 1993 & 117 \\ \text { FY } 1992 & 0 \\ \text { FY } 1991 & 0\end{array}$

$$
09 / 01 / 93-08 / 31 / 94
$$

Objective: To model the subice ocean circulation and melting/freezing rates beneath the Ross and George VI ice shelves.

Product: A better understanding of the influence of seasonal hydrographic variability on the melting and freezing beneath ice shelves and of the impact of these processes on the circulation of shelfwaters.

Approach: Hellmer and Olbers's 2-D slice and channel flow models will be used to investigate thermohaline circulations beneath the Ross Ice Shelf. Existing maps of water-column thickness and depth will define the cavity configuration along potential flow paths. Forcing at the open boundary will be derived from ocean stations and year-long records from mooring arrays. Similar data will be used to validate model results at outflow sites. The initial focus will be on the eastern 
Ross Ice Shelf, where recent glaciological interest and field work has been centered, but application to the larger Ross cavity and to the George VI Ice Shelf is planned. The 3-D techniques being applied to the Filchner-Ronne cavities by European modellers will be studied, and the coupled ice-shelf/ocean modeling proposed by U.S. glaciologists will be considered.

Results to Date: New project.

\section{CLIMATOLOGICAL AEROLOGICAL (UPPER AIR) REFERENCE DATA SET (CARDS)}

KARL, THOMAS R.

\section{NATIONAL CLIMATIC DATA CENTER}

$\begin{array}{ll}\text { FY } 1993 & 450 \\ \text { FY } 1992 & 440 \\ \text { FY } 1991 & 632\end{array}$

$07 / 01 / 92-06 / 30 / 93$

Objective: To build a Climatological Aerological (Upper Air) Reference Data Set (CARDS) from the radiosonde data archives.

Product: A key data set for climate research requiring atmospheric profiles of temperature, winds, and humidity.

Approach: Data on temperature, pressure, wind velocity, and humidity profiles of the atmosphere will be included. Such data have been measured from twice-daily balloon launches at 700 to 800 sites around the globe for about 50 years. However, the data are subject to many biases and are spread over many different media and formats, including the effects of solar radiation on the temperature and humidity sensors. Data are often uncertain and require extensive correction because of inadequate metadata. However, nuch of this data is recoverable.

A global data set of daily upper-air and concomitant surface observations will be produced from various collections of upper-air and colocated surface observations. The most extensive data set possible is being collected. A suite of programs is being developed to correct for random errors and outliers. None of these data sets have been quality controlled in a systematic manner. The procedures will include a range of methods from statistical to physical. Suspect stations have been noted by the U.S. and European weather centers for the past 2 years. This list will serve as a basis for assessing our physical and statistical models for identifying errant observations. The homogeneity of the data from the upper-air network will be assessed, and corrections for biases will be implemented, where appropriate. The station histories will provide a basis to correct for known biases for sensor and method changes and differences over time and between stations. These data will be analyzed with basic climate-change-analysis schemes to help ensure against undetected errors and biases and to provide complete documentation. A modest operational program will be instituted to ensure the integrity of future data. These data sets will be made readily available to users.

Results to Date: A Master Station History (Version 1.0) database has been created. This database is currently undergoing a detailed review, and necessary corrections are being made.

Observational data in a variety of formats have been converted to the CARDS standard format. This effort is approaching $75 \%$ of the expected 25 million observations. Data from the National Climatic Data Center, the National Meteorological Center, the USSR global data set, and several national data sets are included. All data have been sorted to station-number order. The first 5 million observations have been loaded onto an optical jukebox with a relational database management system.

Data are being subjected to standardized quality control before loading. A comprehensive hydrostatic quality control algorithm from the National Meteorological Center has been implemented. Work is under way to develop an advanced and more sophisticated quality control that will be implemented once a kernel database is available.

Research on the detection and methods of correction of biases is under way. One group is researching statistical methods of detection, another is developing physical models based on thermistors, and yet another is developing models for humidity sensors. 


\section{DATA PREPARATION AND ANALYSIS FOR ANNEX III, USA/PRC COOPERATION IN THE FIELD OF ATMOSPHERIC TRACE GASES}

\author{
KARL, THOMAS R.
}

NATIONAL CLIMATIC DATA CENTER
$\begin{array}{cc} & \\ \text { FY } 1993 & 257 \\ \text { FY } 1992 & 244 \\ \text { FY } 1991 & 253\end{array}$

$03 / 15 / 93-03 / 14 / 94$

Objective: To develop comprehensive regional climate reference data sets and to quantify and understand regional climate change in the United States and China by using both observations and GCM calculations (model control climates).

Product: A regional climate data set that can be used as a primary reference for understanding climate change and testing GCMs.

Approach: Updating of the database will be an ongoing effort. Past adjustment schemes must incorporate the new data, and station changes in instrumentation must be evaluated. The effort will include the development of near-real-time updating. The effort will include understanding recent anomalous temperatures that may be real or may be the result of instrumentation changes. This updating includes the temperature, precipitation, water-vapor, sunshine-and-cloud, and station-pressure data sets. Special attention will be given to observations from Alaska, Hawaii, and U.S. island stations.

Land-use information will be obtained to provide a historical data set to determine how use changes may have modified the local microclimate. Such information can be compared with the general land use to determine how representative an observation site is of the surrounding area.

Studies of the urban heat island and the differential changes between day and night temperatures will determine if the rapid rise in nighttime temperatures in the United States and the People's Republic of China are the result of urban contamination of the record or possibly greenhouse-gas-induced changes. Satellite data will be used to assist in the definition of urban/rural sites.
The diurnal temperature pattern from climate-model results will be analyzed. Unique patterns that may provide a fingerprint for detecting climate change will be sought. The NCAR GCM will be used because it is the only model now using most of the greenhouse gases and Wang et al. have shown that including the individual gases (rather than increased $\mathrm{CO}_{2}$ as a surrogate for all greenhouse gases) may result in different regional patterns of climate change.

Model results will be analyzed for changes in circulation patterns as climate changes with slowly increased greenhouse gases. This analysis will check the model's capabilities to represent current patterns as well as provide information for pussible detection of change. The NCAR model will be used again because of the multiple trace gases that are included.

The analysis of the NCAR model will include comparison of the model extreme events to observations. Again, the analysis will check the model as well as provide information for detection strategies. The initial analysis will concentrate on precipitation events, whose representation is a known weakness in climate models.

Multiple changes observed in regional data sets will be studied. For example, a decrease in the diumal temperature range seems to accompany decreased sunshine in China. The analysis will examine if these changes are occurring systematically over a number of regions and if they relate to other changes, such as land use. The initial focus will be on China.

Results to Date: Techniques for detecting and adjusting for undocumented artificial discontinuities in climatic time series were reviewed and tested. Results indicated that the standard normal technique was the most sensitive of those reviewed. A new technique that uses regression and nonparametric methods was developed and proved to perform better than those previously reviewed. This method will be used as a final step in the processing of the U.S. Historical Climatology Network.

Decadal and 20th Century variations of temperature, precipitation (solid and liquid), and snow cover over North America and Asia have been quantified. The most significant finding relates to a Northern Hemisphere increase of minimum temperature relative to the maximum temperature and a general decrease in snow cover, especially during the spring. The decrease in spring snow cover occurs simultaneously with a significant increase in temperature. 
The linkage between snow cover and temperature increase is being explored through surface and space-based data.

A possible discontinuity in the temperature record of National Weather Service first-order stations was examined. Stations that use the HO83 hygrothermometer were found to have a $+0.5^{\circ} \mathrm{C}$ discontinuity in daily maximum temperature when averaged over 190 stations.

Heavy, multiday precipitation events in the Midwest were examined, and synoptic conditions were analyzed with both observed data and a GCM (NCAR CCM1) simulation. Results indicated that these events contribute much to the variance of precipitation in the area but are not well simulated in the model.

\section{ASSESSING THE CLIMATE EFFECT OF CARBON DIOXIDE AND OTHER TRACE GASES WITH AN INTERACTIVE 2-D CLIMATE-CHEMISTRY MODEL.}

KO, MALCOLM K. W.

\section{ATMOSPHERIC AND ENVIRONMENTAL RESEARCH, INC.}

\begin{tabular}{lr} 
FY 1993 & 137 \\
FY 1992 & 0 \\
FY 1991 & 219 \\
& \\
\multicolumn{1}{|c|}{$12 / 01 / 92-11 / 30 / 93$}
\end{tabular}

Objective: To assess the total greenhouse impact of $\mathrm{CO}_{2}$, CFCs, HCFCs, HFCs and $\mathrm{N}_{2} \mathrm{O}$, taking into account the direct effect and the indirect effect from associated changes in other greenhouse gases such as ozone.

Product: A 2-D zonal-mean interactive climate-chemistry model.

Approach: Increases in the concentrations of CFCs, $\mathrm{N}_{2} \mathrm{O}$, and $\mathrm{CH}_{4}$ in the atmosphere can perturb the chemical cycles and modify the distribution of ozone, which is also a greenhouse gas. Surface warming from increases of $\mathrm{CO}_{2}$ and other trace gases could also modify the transport circulation in the lower stratosphere, leading to ozone redistribution. These issues will be investigated with a 2-D zonal-mean interactive climate-chemistry model. The model will be based on coupling two operational models developed at
Atmospheric and Environmental Research. A modular approach will be used so the climate-chemistry model can readily incorporate any refinements to the separate modules. A number of studies will examine the feedback mechanisms in the climate module with emphasis on the effects of tropical clouds and convective adjustment in the presence of ozone redistribution.

Results to Date: The climate module has been extended to a meridional resolution of $10^{\circ}$ latitude, a number of analyses have leen performed to determine how best to parameterize meridional heat transport, and the logic for the interface of the two modules has been finalized.

The existing climate model was used to study the sensitivity of the surface-temperature response to parameterization of tropical convection, parameterization of the optical thickness of cirrus clouds, and the relation between tropical clouds and the sea-surface temperature.

\section{RECENT CHANGES OF WEATHER PATTERNS IN NORTH AMERICA}

\author{
'KUKLA, GEORGE, and KARL, TOM \\ COLUMBIA UNIVERSITY and NATIONAL \\ CLIMATIC DATA CENTER
}

$\begin{array}{ll}\text { FY } 1993 & 233 \\ \text { FY } 1992 & 218 \\ \text { FY } 1991 & 192\end{array}$

$$
08 / 15 / 93-08 / 14 / 94
$$

Objective: To develop the data sets to evaluate changes in the properties and frequencies of air masses and extreme weather events over North America and to compare any observed changes to climate-model predictions of greenhouse-gas- and aerosol-induced climate change.

Product: Data on climate short-term variability and on changes in climate variability and extreme weather events.

Approach: The air mass types will be defined primarily on humidity-and wind-related parameters. The daily frequencies of air mass types will be analyzed for relationships to extreme warm/cold and El Niño/La Niña years. The variability and changes within individual airmass types will be documented, and the temperature extremes within an air mass will be analyzed. The 
results of the preceding analyses will be compared with climate-model calculations.

The following questions will be addressed. Are there any systematic time-related changes in the air-mass frequencies in North America? If so, how do they compare to model calculations? Are there any systematic time-related changes in the temperature of comparable air-mass types? If so, how do they compare to model calculations? Are there any changes in the precipitation patterns over North America, and do they relate to the changing frequency patterns, persistence, or character of the air-mass types?

Results to Date: The most striking and widespread change of surface air temperature over much of North America in recent decades is the increase of nighttime temperatures and the decrease of the diurnal temperature range. We have found similar trends accompanied by only little rise, or even by a decrease, of the daily maximum temperature over several other regions of the Northern Hemisphere.

An international confcrence sponsored by NOAA and DOE has been organized to further study this phenomenon and to test whether the effect of $\mathrm{CO}_{2}$ on nighttime temperatures combined with $\mathrm{SO}_{x}$ impact on daytime temperatures can explain the observed trends.

Modeling of the daily temperature structure has been found to need improvement because the comparison of observed with modeled data for the central part of North America has shown large differences.

\section{CARBON DIOXIDE EFFECTS RESEARCH}

MacCRACKEN, MICHAEL C.

\section{LAWRENCE LIVERMORE NATIONAL LABORATORY}

$\begin{array}{ll}\text { FY } 1993 & 659 \\ \text { FY } 1992 & 908 \\ \text { FY } 1991 & 988\end{array}$

$10 / 01 / 91-09 / 30 / 92$

\section{Task 1: Climate Modeling (Karl E. Taylor, Principal} Investigator)

Objective: To sharpen estimates of future climate change by determining the response to prescribed radiative perturbations and by increasing understanding of complicating factors, such as cloud-feedback effects and stratospheric volcanic aerosols.

Product: Improved understanding of the processes that determine climate sensitivity and maintenance and enhancement of current models of the atmosphere and oceans.

Approach: Tools that have been developed at Lawrence Livermore National Laboratory (LLNL) and other research centers during the past decade will be built upon. In particular, 3-D atmospheric GCMs and simplified ocean and sea-ice models will be used to study issues of climate sensitivity and feedbacks. The models will be compared with new observations (and other, similar models) through participation in such programs as FANGIO (Feedback Analysis for GCM Intercomparison and Observation) and PMIP (Paleoclimate Modeling Intercomparison Project). New capabilities needed to simulate the seasonal cycle and to properly account for the effects of aerosols will be added to the GCM for this research.

The GCMs will be used to analyze in detail the relative importance of the various processes and feedbacks (such as changes in sea ice) that contribute to the spatial and seasonal sensitivity of climate to the radiative perturbations expected from an increase in atmospheric $\mathrm{CO}_{2}$ and sulfate aerosols. A GCM will be used to try to reproduce some of the climate changes that occurred thousands of years ago. Where possible, results will be compared with observations.

The transient behavior of the climate system will be studied with two global ocean models adapted to the LLNL computer system, an isopycnal coordinate model developed by Oberhuber and Semtner and Chervin's more traditionally formulated ocean GCM (OGCM).

The comprehensive GCMs will be used to analyze in detail the relative importance of the various processes and feedbacks that contribute to the spatial and seasonal sensitivity of climate to radiative perturbations similar to those expected from an increase in atmospheric $\mathrm{CO}_{2}$. The zonally averaged model and simplified models of individual processes will then be used to assist in analyzing and interpreting results. They can also be used in exploratory studies to help determine which lines of research are likely to be most fruitful with the GCMs. Where possible, results will be compared with observations. 
Results to Date: A simulation has been completed, consistent with the current FANGIO experiment, that involves a comparison of the seasonal cycle of cloud radiative forcing produced in the model with that observed by the Earth Radiation Budget Experiment (ERBE). In the zonal and annual average, the model compares favorably with observations, and the seasonal cycle of the zonally averaged cloud radiative forcing is also qualitatively consistent with the data. The amplitude of the seasonal cycle of cloud radiative forcing is, however, generally too weak by a factor of two or three. Further analysis is under way to uncover the reasons for this discrepancy.

A capability has been added to the GCM so that it can now simulate climatic response to increasing concentrations of atmospheric aerosols (either volcanic or anthropogenic) as well as compute the radiative forcing attributable to these aerosols. A mixed-layer ocean model and a simple sea-ice model have also now been included in this model to allow true climate-change experiments. Experiments have been conducted to calculate the climatic effects of the El Chichon volcanic eruption and to estimate the effects of sulfate aerosols. Preliminary results indicate that, if sea-surface temperatures are held fixed, the tropospheric-circulation changes associated with El Chichon are weaker than had been suggested previously by others.

Task 2: Program Management (M. C. MacCracken, Principal Investigator)

Objective: (1) To prepare technical reports and presentations providing state-of-the-art estimates of the climatic effects of increasing $\mathrm{CO}_{2}$ and the extent that such changes have started to occur; (2) to assist in providing technical evaluation and coordination of the climate-research component of the DOE GCRP and in integration of the program with the USGCRP; (3) to foster graduate and postdoctoral education through sponsorship of collaborative research within the University of California system of campuses and laboratories; and (4) to sponsor special enabling research to assist the DOE GCRP.

Product: Coordination of DOE programs with federal and international programs relevant to the effects of $\mathrm{CO}_{2}$ and other greenhouse gases on climate; specific studies on timely $\mathrm{CO}_{2}$-climate issues; and statisticalanalysis support for such activities as overviews of model-to-data comparisons.
Approach: A scientific advisor will assist the DOE GCRP in the formulation and implementation of the DOE research program, review of research proposals and scientific literature, preparation of progress reports, planning of meetings, interactions with the USGCRP, and communication with the scientific community. Under DOE guidance, LLNL will subcontract to other investigators to initiate studies in innovative technical areas. This project will be particularly responsive to the varying needs of the DOE Environmental Sciences Division and will be encouraged to foresee and investigate potential new areas of concern and interest.

Results to Date: With DOE encouragement, significant assistance has been provided in managing the USGCRP. From February through June, assistance was provided to the Integrative Modeling and Prediction Working Group of the USGCRP in moving toward a plan to fulfill its responsibilities for encouraging development of Earth system models. Beginning in August, the directorship of the new office of the USGCRP was assumed, with major responsibility for overseeing development of the multiyear research plan.

Matching support was provided for graduate and postdoctoral collaborative research within the University of California multicampus system, particularly in support of research interactions with the Scripps Institution of Oceanography and UCLA.

Support was provided to Lamont-Doherty Earth Observatory in the development of an isotopic database for use in verification of ocean GCMs.

Task 3. Global Climate Change Curriculum Development (Manuel Perry, Principal Investigator)

Objective: To provide elementary-, middle-, and highschool teachers with the support necessary to develop effective, interdisciplinary instructional materials related to the problem of $\mathrm{CO}_{2}$ buildup in the Earth's atmosphere.

Product: Multidisciplinary curricular material for high-, middle-, and elementary-school grade levels.

Approach: Curriculum materials on global climate change will be developed and disseminated. These materials will stress the interdisciplinary nature of the issues but will focus on the relevant scientific aspects. The scientific, technical, and administrative resources of LLNL will be tapped along with the experience of 
currently active teachers to ensure cooperative and effective implementation of the curriculum materials. These materials will be distributed through teacher workshops that will help train teachers to use them in classrooms and schools. College credits will be provided for teachers attending the workshops. By bringing together teachers of both scientific and nonscientific disciplines to focus on a problem of international concern, this program will create a setting in which the sciences are seen as relevant and beneficial to humanity. The program will also inspire a valuable partnership between the scientific and educational communities.

Results to Date: About 75 high-school teachers participated in three 3-day interdisciplinary workshops in 1993. The scope of the program increased this year to encompass states in other parts of the country. Two of the workshops were at Souther. University in Louisiana, and one was at Norfolk State University in Virginia. At the middle-school level, teachers led a workshop for students at the University of the Pacific, in Stockton, California. The 7th and 8th graders attending this workshop were primarily low-income and minority students. The elementary-school teachers led two workshops for the Livermore Association for Guiding and Teaching Students.

GLACIER CALVING, DYNAMICS, AND SEALEVEL RISE

\section{INSTITUTE OF ARCTIC AND ALPINE RESEARCH}

MEIER, M. F., and PFEFFER, W. TAD

$\begin{array}{lr}\text { FY } 1993 & 214 \\ \text { FY } 1992 & 0 \\ \text { FY } 1991 & 0\end{array}$

09/01/93-08/31/94

Objective: To develop numerical models to simulate iceberg calving and to shed light on the role of calving in ice dynamics.

Product: A numerical model of glacier dynamics that includes physics-based interaction between ice and water at a calving front.

Approach: The current calving flux from Greenland and Antarctica is very poorly known, contributing a significant portion of the uncertainty in the current mass balance of these ice sheets and in the prediction of the response of glaciers and ice sheets to changing climate. Work from four research areas will improve the ability to predict the future contribution of glaciers to sea level: remote-sensing observations of calving activity and iceberg flux, numerical modeling of glacier dynamics, theoretical analyses of the calving process, and numerical techniques for modeling flow with large deformations and fracture. These four areas will be combined into a single research effort, and calving dynamics will be explicitly included in a model of glacier dynamics. The application of this model will allow much more realistic simulations of interactions between glacier dynamics, glacier mass balance, and sea-level change.

Results to Date: New project.

\section{CLOUDS AND OCEAN-ATMOSPHERE INTERACTIONS}

\author{
RANDALL, DAVID A. \\ COLORADO STATE UNIVERSITY
}

$\begin{array}{ll}\text { FY } 1993 & 185 \\ \text { FY 1992 } & 190 \\ \text { FY 1991 } & 174\end{array}$

09/15/93-09/14/94

Objective: To test the GCM against observations, to understand the role of clouds in climate, and to develop and understand coupled models of the atmospheric and ocean system.

Product: Improved understanding of the role of clouds in climate and climate change and an improved coupled atmosphere-ocean GCM tested against observations.

Approach: An intercomparison will be participated in, and the analysis and diagnosis of snow feedback will be led. The initial forcing for a doubling of $\mathrm{CO}_{2}$ will be calculated as part of the intercomparison to see if the basic radiation codes in each model provide the same initial increase in radiative forcing.

The Colorado State University (CSU) atmospheric model will be coupled with the Semtner-Chervin ocean model in a step-wise process to ensure an understanding of the results. Climate experiments will be carried out with slowly increasing concentrations of atmospheric 
$\mathrm{CO}_{2}$. The model calculations will be compared with observations to diagnose the issues of the exchange of heat, moisture, and momentum at the air-sea interface.

To assist in the investigation of clouds, the model will be run in an all-land mode. One of the major problems in attempting to understand the role of clouds in climate and climate change is that a change in another parameter (e.g., snow) often changes clouds. Multiple feedbacks occur and complicate the diagnosis. This variation on an old idea will attempt to circumvent much of the problem.

The CSU model will be compared to the seasonal ERBE data, which provide a measure of how clouds presently affect the Earth's radiative balance. This comparison will test the model's radiative code, and cloud-parameterization code.

Results to Date: The results of the diagnostic study of snow feedback show that the temperature-dependence of the snow albedo can lead to a spurious negative snow feedback in some models. The simulations for the $\mathrm{CO}_{2}$ forcing study have also been carried out and provided to FANGIO. A new control run with the coupled oceanatmosphere model is currently in its fourth simulated year. The GCM results with ERBE data have been published. Simulations of the Earth's entropy budget are currently being compared with satellite observations. An analysis of the AMIP simulations shows that errors in the implied ocean energy transports, present in most of the models' results, are mainly caused by errors in the Earth's radiation budget.

\section{METHANE FLUXES FROM RICE FIELDS IN CHINA: SPATIAL AND TEMPORAL VARIABILITY AND ESTIMATES OF CONTRIBUTIONS TO THE GLOBAL BUDGET}

\author{
RASMUSSEN, R. A., and KHALIL, M. A. K. \\ OREGON GRADUATE INSTITUTE
}

$\begin{array}{lr}\text { FY } 1993 & 410 \\ \text { FY } 1992 & 393 \\ \text { FY } 1991 & 330 \\ & \\ & 02 / 01 / 93-01 / 31 / 94\end{array}$

Objective: To measure the magnitude of the methane emission from rice-paddy fields.
Product: Data on the magnitude of methane emissions from rice-paddy fields, including seasonal and geographic characteristics.

Approach: The Oregon Graduate Institute will cooperate with the Chinese Academy of Science (Institute of Atmospheric Physics) to measure the methane released to the atmosphere from rice-paddy fields. The measurement approach has been reviewed and tested extensively in field during the past 25 years. Basically, a frame will be placed in the field as a seal for the enclosure. The enclosure will be placed over the plants, and a sample will be taken quickly, with little or no disruption to the plant or the surrounding surface. The technique is portable, easily field-maintainable, and provides reproducible data. Air and soil temperature, growth stage, soil type, fertilizer type, farming practice, etc. will be recorded.

The baseline effort at $\mathrm{Tu} \mathrm{Zu}$ will continue. This will provide a 6-year base of measurements that can be compared with data taken at other locations. These additional sites will be selected to investigate spatial variability from different climate zones, soil types, farming practices, and rice varieties. The data, along with other methane budget data, will be used to estimate seasonal variations and geographic variations in the global methane budget.

Results to Date: Studies of most of the extrinsic factors (soil temperature, meteorology, agricultural practices, and the effect of pests and diseases) and some of the intrinsic factors (rice cultivar, soil type, microbial ecology, root exudates, $\mathrm{pH}$, and soil electropotential) indicate that methane emissions increase with increasing soil temperature, wind speed, and plant density; emissions decrease under cloudy conditions or when there is little standing water. The established factors have been included in the standard methods of the Intergovernmental Panel on Climate Change (IPCC) partly as a result of this research. This understanding allows a more accurate evaluation of the entire global methane budget.

The data were used to evaluate the historical budget of met iane from China to find that methane emissions may now be significantly less than those a decade ago. 


\section{VALIDATYON OF SOIL MOISTURE IN GCMs - AMIP DIAGNOSTIC SUBPROJECT 11}

\author{
ROBOCK, ALAN
}

\section{UNIVERSITY OF MARYLAND}

$\begin{array}{lr}\text { FY } 1993 & 50 \\ \text { FY } 1992 & 0 \\ \text { FY } 1991 & 0\end{array}$

$09 / 01 / 93-08 / 31 / 94$

Objective: To collect, digitize, and analyze soilmoisture measurements from selected areas in Russia, China, and the United States and to use these data for the validation of the soil-moisture simulations from the atmospheric GCMs in the AMIP.

Product: A soil moisture data set for GCM validation.

Approach: Under DOE sponsorship, AMIP is using 29 GCMs to simulate the climate of the decade 1979 to 1988. The simulations of soil moisture in these models will be investigated, and model simulations of the climatology of the seasonal cycle as well as interannual variability will be validated. Soil moisture is a critical GCM diagnostic for the assessment of drought frequency and severity and for the terrestrial component of the surface energy budget.

Soil-moisture measurements from selected areas in Russia, China, and the United States will be collected, digitized, and analyzed. Subsequently, the data will be used for the validation of the soil-moisture simulations from the atmospheric GCMs in the AMIP.

Results to Date: New project.

\section{PARTIAL SUPPORT OF RESEARCH ON GREENHOUSE-GAS-INDUCED CLIMATE CHANGE}

SCHLESINGER, MICHAEL E.

\section{UNIVERSITY OF ILLINOIS}

$\begin{array}{lr}\text { FY } 1993 & 150 \\ \text { FY } 1992 & 0 \\ \text { FY } 1991 & 0\end{array}$

$06 / 15 / 93-06 / 14 / 94$
Objective: To continue the development and application of the Illinois GCM; to analyze feedbacks and climate forcings, such as stratospheric ozone feedback and aerosol climate forcing; to examine the model simulation of the past 100 years and over the end of the last glaciation; and to estimate feedbacks between climate and the methane cycle.

Product: Increased confidence in the GCM and understanding of climate-feedback mechanisms.

Approach: Two 100-year simulations will be performed with the new OGCMs and the new atmospheric GCMs (AGCMs) to simulate the past 100 years of climate. The control simulation will use fixed radiative forcing, and the experiment will use the observed changes in trace species, including $\mathrm{CO}_{2}$ and sulfate aerosols. The paleoclimate simulations will continue. The model will be applied to the end of the last glaciation. The methane cycle will be analyzed to ascertain the feedbacks between climate change and the methane cycle. The improved model will be used to analyze the global warming/stratospheric ozone feedback, particularly polar stratospheric clouds (PSCs). The hypothesis is that global warming may increase moisture and decrease stratospheric temperatures sufficiently for PSCs to form in the tropics. The GCMs will be formulated and tested on the CM-5. Increased model resolution may then be possible to directly address regional scale climate issues of interest to policy analysts.

Results to Date: A parameterization was develped for cloud evaporation for AGCMs; the radiative-transfer model for our multilayer AGCM was improved; the soil-moisture parameterization was revised for our two-layer and multilayer AGCMs; a dynamic sea-ice model was developed for our OGCM; the OGCM's vertical resolution was doubled to 12 layers; our multilayer AGCM was revised to reduce its computational requirements; and development was begun on a tropospheric-stratospheric GCM.

The coupled atmospheric general circulation/mixed-layer ocean/ice-sheet/asthenosphere model was used to simulate the onset of the last glacial climate from $115,000 \mathrm{BP}$ to $105,000 \mathrm{BP}$ and the glacialogical and thermal-expansion changes in sea level induced by a $\mathrm{CO}_{2}$ doubling. A 58-year oscillation in the observed global-mean temperature record was discovered.

In collaboration with Lamont-Doherty Earth Observatory and the National Climatic Data Center, GCM-simulated 
current diurnal temperature cycles in the U.S. midwest were compared with observed values.

Cause-and-effect analysis (CEA) was used to analyze feedbacks in geophysical models. The zero-feedback response of the climate system to external forcing was analyzed, and a heretofore-overlooked feedback mechanism was discovered. The proper use of the adjoint method to analyze the sensitivity and uncertainty of climate-model simulations was described.

The influence of sulphates on climate sensitivity was analyzed; the influence of the Sun and sulphates on climate sensitivity was investigated; research with the Institute of Experimental Meteorology in Obninsk on the influence of volcanoes on global-mean temperature was continued; and collaborative research on the contribution of volcanoes to El Niños was initiated.

Estimation of the $\mathrm{CO}_{2}$-induced climate change at six stations in Norway has been continued with the climatological-projection-by-model-statistics technique.

\section{PARTIAL SUPPORT FOR THE NAS BOARD ON ATMOSPHERIC SCIENCES AND CLIMATE}

$$
\text { SPRIGG, WILLIAM }
$$

\section{NATIONAL ACADEMY OF SCIENCES}

$\begin{array}{lr}\text { FY 1993 } & 53 \\ \text { FY 1992 } & \mathbf{0} \\ \text { FY 1991 } & 0\end{array}$

$$
\text { 09/01/93-08/31/94 }
$$

Objective: To provide scientific leadership and guidance to the government on important national and international issues in the atmospheric sciences and climate.

Product: Regularly scheduled board, committee, and panel meetings that will review current national and international issues and provide advice and assistance to the government as needed.

Approach: The Board on Atmospheric Sciences and Climate will provide scientific leadership and guidance to the government on national and international issues in the atmospheric sciences and climate and on the coordination between discipline-oriented activities within the atmospheric sciences and interdisciplinary programs, such as the International Geosphere-Biosphere Program (IGBP).

It will maintain an overview of science issues and implications of atmospheric chemistry in the earth and environmental sciences through the Committee on Atmospheric Chemistry (CAC). CAC will focus on understanding atmospheric properties (such as cloud processes) and contemporary atmospheric changes (such as increases in greenhouse gases, depletion of stratospheric ozone, acid deposition, and air pollution).

The Boand will respond rapidly and effectively to requests by federal agencies and Congress for evaluations, guidance, recommendations, and support in research and applications in the atmospheric and climate sciences.

\section{STUDY OF CLIMATE VARIABILITY ON TIME SCALES OF DECADES TO CENTURIES}

SPRIGG, WILLIAM A.

\section{NATIONAL ACADEMY OF SCIENCES}

$\begin{array}{lr}\text { FY } 1993 & 25 \\ \text { FY } 1992 & 0 \\ \text { FY } 1991 & 0\end{array}$

$$
\text { 01/01/93-12/31/93 }
$$

Objective: To conduct an assessment of climate variability on 10 - to 100 -year time scales.

Product: A useful reference in research to verify climate models and diagnostic studies of climate and to distinguish natural from anthropogenic causes of climate change.

Approach: A workshop held September 21-25, 1992, at Irvine, California, was structured to examine evidence for 10- to 100-year climate variability in the instrumented atmospheric and oceanographic record; proxy data on decadal climate variability from ice cores, tree rings, ocean sediments, and coral growths; modeling of atmospheric and ocean responses to sea-surface temperature, salinity, and wind variations; and simulating climate variations in coupled models.

The Climate Research Committee will review, edit, and compile contributed papers, as amended during the workshop, along with workshop discussions into a 
comprehensive report with an overview and recommendations. The draft will be subjected to peer review by the National Research Council.

Results to Date: New project.

\section{STUDY OF REGIONALL CLIMATE CHANGES}

WANG, WEI-CHYUNG

\section{STATE UNIVERSITY OF NEW YORK AT ALBANY}

$\begin{array}{lr}\text { FY } 1993 & 373 \\ \text { FY } 1992 & 326 \\ \text { FY } 1991 & 0\end{array}$

$01 / 01 / 93-12 / 31 / 93$

Objective: To develop quality-assured data to study long-term regional climate change; to validate climate models; and to understand the differences among the climate models and to improve them by incorporating advanced radiative submodels that include $\mathrm{CO}_{2}$ and other greenhouse gases.

Product: Regional and global climatic data necessary to document climate change and to assess natural variability and databases with which to test climate models and to improve the basic model parameterizations.

Approach: Two DOE GCM intercomparisons will be participated in. Model simulations of the period from 1979 to 1988 will be conducted for AMIP with the CCM1. The fixed-sea-surface-temperature experiments will be conducted for the other intercomparison.

Historical and instrumental climate data sets for the United States and for China continue to be collected and examined. The documentation and quality control will be completed prior to the general release of the data. The research necessary to incorporate the proxy (paleoclimate data), historical writings, and instrumental data into a quantitative time series will be initiated. Such data can then be used to investigate long-term natural climate variability and to validate very long GCM runs.

The regional climate data base (United States and China) will be examined to elucidate regional climate, the pattern and timing of changes, comparisons across regions, and the regional character of the urban heat island.
How best to bring the data and the models together will be explored. The NCAR CCM1 will be extended to differently forced climates, and low-frequency climate events, such as the El Niño/Southern Oscillation and the Northern Oscillation, will be investigated. The regionalscale relationships within the data will be compared with the GCM results. These studies will assist in validating GCMs and will provide information for future predictability studies.

Results to Date: We continued participating in AMIP/FANGIO. Two National Center for Atmospheric Research (NCAR) GCMs, the CCM1 and the GENESIS, are being used to conduct climate simulations with specified 1979-1992 sea surface temperature. In the CCM1 simulation, the model catches the major observed features of anomalous precipitation patterns of summer monsoons in China during the 1982-1983 and 1986-1987 El Niño events; these features include floods in the Yangtze River Valley and droughts in Southeastern China. The GENESIS simulation examines the effect on climate simulations of a new ozone climatology derived from TOMS, SAGE, and ozonesondes. Preliminary results suggest that the temperature structure is quite different between the new ozone data set (which includes the longitudinal variation) and the old ozone data set (which considers the zonal mean). Diagnosis of the seasonal cloud-radiative forcing was begun, and participation in IPCC WG 1 was continued.

Beijing cloudiness was reconstructed and analyzed in a two-part study. The first examined the interannual variation of the observed cloudiness, solar radiation reaching the surface, surface air temperature, precipitation, humidity, and number of rain-days for the period 1951-1990, and the second reconstructed the cloudiness for the period $1875-1950$ with the rain-day information contained in the Clean-Rain-Records.

The monsoon circulation and its association with lowfrequency oscillations in the low- and mid-latitude ocean-atmosphere system was studied by analyzing both the observations and GCM simulations. Two studies were conducted. The first studied the Asia monsoon movement with the satellite-measured outgoing longwave radiation. The second developed a pair of indices to reflect the location and intensity of the summer monsoon in China. Those indices can be used to quantify the ability of a GCM to simulate the monsoon.

The internannual variability and the changes to it caused by the greenhouse effect was studied by analyzing two 
100-year GCM simulations of the present climate and climate warming caused by the enhanced greenhouse effect. Comparisons of the variability between the model present climate and the observed climate were also conducted. The results suggest that the model is capable of simulating some of the observed large-scale features, such as the global and hemispheric mean surface air temperature and Arctic sea-ice extent. However, it is less satisfactory in simulating the climate variability, especially over the land areas. ।

\section{ENHANCED RESEARCH PROGRAM ON THE LONG-RANGE CLIMATIC EFFECTS OF INCREASING ATMOSPHERIC CARBON DIOXIDE}

\author{
WASHINGTON, W. M., and MEEHL, G. \\ NATIONAL CENTER FOR ATMOSPHERIC \\ RESEARCH
}

$\begin{array}{lr}\text { FY } 1993 & 426 \\ \text { FY 1992 } & 0 \\ \text { FY 1991 } & 0\end{array}$

06/01/93-05/31/94

Objective: To couple the enhanced atmospheric CCMO and CCM2 and the $1^{\circ} \times 1^{\circ}$ dynamical ocean/sea-ice GCM and to perform a series of sensitivity experiments with the coupled and uncoupled models, including transient- $\mathrm{CO}_{2}$ experiments.

Product: A new global coupled GCM capable of improved regional climate simulations, especially those involving ocean and sea-ice dynamics.

Approach: A control run $\left(1 \times \mathrm{CO}_{2}\right)$, an instantaneous $2 \times \mathrm{CO}_{2}$ experiment, and a transient experiment will be performed with the enhanced atmospheric GCM (CCM0 and CCM2) to benchmark the new version of the model against the previous results.

The two advanced models will be coupled. The dynamical ocean model will be a $1^{\circ} \times 1^{\circ}$ version of the Semtner-Chervin $1 / 2^{\circ} \times 1 / 2^{\circ}$ ocean model with 20 vertical levels that explicitly resolves some aspects of the mesoscale eddies as does the parent model. Research has shown that observed ocean features sufficient for climate experiments can be simulated without such a detailed, explicit calculation. That hypothesis will be tested with the coupled model.

The coupling scheme is synchronous. (Asynchronous coupling does not allow for the time evolution of the interaction between the ocean and atmosphere.) In the NCAR scheme, the atmospheric model provides wind stress, precipitation minus evaporation, and the sum of the surface energy balance to the ocean. The ocean provides surface temperature and sea-ice distribution to the atmosphere. The coupled experiment is started from separate runs of the new models, each forced with the appropriate observed climate conditions (e.g., the observed sea-surface temperature for the spinup of the atmospheric model). This practice allows separate diagnosis of any model problems before coupling.

The result will be the first coupled GCM experiments with a medium-resolution ocean model $\left(1^{\circ} \times 1^{9}\right)$ and dynamic sea ice sufficient to simulate primary ocean climatological features.

Results to Date: The coupled model is now being tested on multidecadal runs. We can keep the climate reasonably close to that observed without the normal flux-correction methods. The ocean model components make use of the Semtner-Chervin model at $1^{\circ}$, and the sea ice uses the Flato-Hibler dynamic method and the improved thermodynamics approach of Semtner. Coupling to the recently released CCM2 is under way. Changes in interannual variability (as shown by empirical orthogonal function) are being studied, changes in the interannual variability are being compared to microwave-sounding-unit data, and the mixed-layer and coupled-model changes in variability are being compared.

\section{SENSITIVITY OF CLIMATE MODELS: COMPARISON OF SIMULATED AND OBSERVED PATTERNS FOR PAST CLIMATES}

\author{
WEBB, THOMPSON, III; KUTZBACH, J. E.; and \\ OGLESBY, R. J.
}

BROWN UNIVERSITY, UNIVERSITY OF . WISCONSIN, and PURDUE UNIVERSITY

\begin{tabular}{lr} 
FY 1993 & 450 \\
FY 1992 & 425 \\
FY 1991 & 400 \\
\multicolumn{2}{r}{$02 / 01 / 93-01 / 31 / 94$}
\end{tabular}


Objective: To validate the NCAR GCM against paleoclimate data.

Product: A comparison between the paleoclimate data and the NCAR GCM.

Approach: General circulation models will be used to simulate a series of climatic changes from the past. Paleoclimatic data from large areas of the tropics and the northern hemisphere mid-to-high latitudes will be asembled for the comparison of model-simulations and observations. Methods and procedures will be developed for improving the comparability of the observed and simulated data. And the model results and sensitivity studies will be compared with the paleoclimatic data.

Results to Date: CCM1 seasonal simulations of modern and mid-Holocene (6000 BP), a target for the Paleoclimate Modeling Intercomparison Project (PMIP), showed that the northern tropics became even wetter and central North America even drier 6000 ago and were similar to the paleoclimate data. The CCMO simulation did not match the data. CCM1 estimates of modern runoff and river discharge into the ocean basins agreed well with observations and were also calculated for the last glacial maximum and for $6000 \mathrm{BP}$ simulations.

Marine data-model comparisons were made for upwelling in the Arabian Sea, SSTs off southeast Africa, and monsoon wind intensity. CCM1 sensitivity studies examined changes in $\mathrm{CO}_{2}$, solar constant, orbital parameters, the height of mountains and ice sheets, Indian Ocean SST, and vegetation. CCM1 was also coupled to a more physically realistic snow hydrology and moresophisticated mixed-layez ocean and sea-ice models.

\section{DETECTION OF $\mathrm{CO}_{2}$-INDUCED CLIMATE CHANGE}

WIGLEY, T. M. L., and JONES, P. D.

UNIVERSITY OF EAST ANGLIA

$\begin{array}{lll}\text { FY } 1993 & 176 \\ \text { FY } 1992 & 168 \\ \text { FY } 1991 & 118\end{array}$

$12 / 01 / 92-11 / 30 / 93$

Objective: To assemble and analyze instrumental climate data and to develop and apply climate models as a basis for detecting greenhouse gas-induced climatic change and validating GCMs.

Product: A high-quality, spatially extensive database to define the noise of natural climatic variability and its spatial characteristics.

Approach: Global surface climate data bases will be expanded and updated with the extensive resources available to the Climatic Research Unit. Data analyses will focus on the use and development of appropriate statistical, techniques for signal detection and pattern recognition. Interpretations will be guided by appropriate climate models.

Results to Date: Our updated land/marine temperature data set shows a recent cooling of $0.4^{\circ} \mathrm{C}$ for the Northern Hemisphere and $0.2^{\circ} \mathrm{C}$ for the Southern Hemisphere, probably attributable to the eruption of Mt. Pinatubo. Cooling is most pronounced in the Northern Hemisphere in the summer months.

New land-based maximum- and minimum-temperature compilations show that minima have warmed some three times faster than maxima.

Paleoclimate data have been analyzed to assess their value in detection studies. A new compilation suggests that the Northern Hemisphere is currently warmer than at any time since $A D 1500$.

The small glacier contribution to past sea-level rise has been reassessed at $10 \mathrm{~mm}$ between 1900 and 1961, much less than Meier's (1984) estimate of $28 \mathrm{~mm}$. Together with studies with a newly developed smallglacier model, this result indicates that projections of future sea-level rise from small glaciers may have to be revised downward.

To estimate future radiative-forcing changes and their uncertainties, a new methane model has been developed, and our carbon cycle model has been improved. The forme gives a preindustrial natural emissions level of 220 to $330 \mathrm{TgCH}_{4} /$ year, substantially more than the best (IPCC) estimate of the current natural level. The latter has been used to determine the emissions required to stabilize future $\mathrm{CO}_{2}$ concentrations. Warming and sealevel-rise implications of $\mathrm{CO}_{2}$ stabilization have been calculated. 
TRACE-GAS INTERACTIONS IN THE GLOBAL ATMOSPHERE

\author{
WUEBBLES, DONALD J. \\ LAWRENCE LIVERMORE NATIONAL \\ LABORATORY
}

$\begin{array}{ll}\text { FY } 1993 & 385 \\ \text { FY } 1992 & 318 \\ \text { FY } 1991 & 205\end{array}$

$10 / 01 / 92-09 / 30 / 93$

Objective: To understand the effects that increasing atmospheric concentrations of $\mathrm{CO}_{2}$ and other trace gases may be having on global atmospheric chemistry and climate; to understand the interactions among climate, chemical processes, and radiative processes; and to establish clear relationships between theoretical models and atmospheric-constituent measurements made in the troposphere and stratosphere.

Product: A better understanding of global atmospheric chemistry and climate processes; insights into the determination and evaluation of the mechanisms and processes reflecting the influence that trace gasses may be having on the global environment; and an understanding of what measurements are required to further develop and validate global atmospheric models to study climate and chemical interactions.

Approach: Information on past trends and the potential for future increases in the concentrations of various non$\mathrm{CO}_{2}$ trace species will be developed. The budget of the emissions, sources, and sinks of these gases will be analyzed to improve the capability for projecting future concentrations of these gases. Model-sensitivity analyses will be performed to better define the mechanisms, key parameters, and overall uncertainties of potential trace-gas influences on the atmosphere. To define and reduce existing uncertainties in the determination of trace-gas effects, a clearer relationship will be established between theoretical models and the measurements of atmospheric constituents being made in the troposphere and stratosphere. To accomplish this evaluation, LLNL's present capabilities for modeling global atmospheric chemical, radiative, and physical processes will be extended, and new models will be developed to allow coordinated treatment of chemical and climatic effects.
The LLNL 1-D and 2-D atmospheric-chemistry, radiation-transport models have been reference models for past assessments. Results of atmospheric-chemistryperturbation studies with the 2-D model are being used to determine the global-average and latitude-dependent direct and indirect radiative perturbations. The climatic significance of these radiative changes can then be evaluated with climate models. More accurate evaluation of the coupling among trace-gas concentrations, radiative and dynamical perturbations, and climate will be possible with the new 3-D chemical model.

Results to Date: Model development to further reduce uncertainties and increase model capabilities was continued. The treatment of dynamical and radiative processes were improved in the 2-D model. The evaluation of global warming potentials, including the indirect effects on climate forcing resulting from chemical interaction, were improved. The relationship between observed ozone and temperature in the lower stratosphere was evaluated. The impact of the Mt. Pinatubo volcanic eruption on the global atmosphere was assessed.

The treatment of atmospheric dynamics and transport in the 2-D model is being improved with the goal of producing an internally consistent, fully coupled model of these processes. A major step has been the development of a first-principle treatment of atmospheric waves. Additional improvements will replace the current perturbation approach used for temperature feedback with a direct time-integration temperature calculation. We developed algorithms for solar radiative transfer to improve the accuracy of photolysis-rate calculations near twilight. The Arakawa-Schubert convective parameterization was added to our 1-D model, completing development of a single-column radiative-convective-chemistry model useful for detailed diagnostics of radiative and chemical perturbations. This adds to our capabilities to evaluate radiative forcing of trace gases after chemistry feedbacks and stratospheric temperature equilibration. The numerical methods used to solve the large set of stiff ordinary equations for kinetics reactions and transport in the multidimensional models are being improved. Model verification and mechanistic studies continued to define model sensitivities and uncertainties.

The treatment of cloud processes and heterogeneous chemistry was studied to determine the importance of these processes in climate change. Our research studies related the effects of heterogeneous chemistry on aerosols and their likely significant importance in 
explaining the ozone decreases occurring in the lower stratosphere. The linkage between the observed ozone changes and their potential effects on climate change was also examined. More tropospheric chemistry was implemented in the 2-D model. Further validation of tropospheric chemistry and its effects on ozone was performed as an initial step in studying the interaction of tropospheric chemistry and climate.

A chapter on the effects of methane on the global environment, particularly on climate and ozone, was prepared for a NATO-sponsored book assessing the current understanding of atmospheric methane. Contributions were made to the section on radiative forcing in the new IPCC supplemental assessment on climate change. A preliminary analysis of the radiative forcing effects from methane-induced increases in stratospheric water vapor was conducted. Lower-stratospheric ozone concentration was compared with observed and calculated temperature trends.

\section{Computer Hardware, Advanced Mathematics, and Model Physics (CHAMMP) Program}

\section{AN EXPERIMENTAL REGIONAL-SCALE CLIMATE-SIMULATION LABORATORY}

ANDERSON, JOHN R.

\section{UNIVERSITY OF WISCONSIN}

$\begin{array}{lr}\text { FY 1993 } & 104 \\ \text { FY 1992 } & 100 \\ \text { FY 1991 } & 0\end{array}$

$08 / 01 / 93-07 / 31 / 94$

Ohjective: To develop new numerical methods and model formulations for both atmospheric and ocean GCMs suitable for massively parallel computers.

Product: New geophysical fluid dynamics algorithms.

Approach: An evaluation will be made of the local spectral technique for formulating the atmospheric equations of motion in a GCM. This method has yet to be thoroughly tested. It has promising scaling characteristics for deployment on massively parallel computers.

A reformulation of the ocean-modeling equations will be made with three alternative methods that are designed to make the numerical solution of the equation set computationally more efficient. The various methods will be compared against current methods for efficiency and accuracy.

These new methods will first be deployed on the prototype computer system built at the University of Wisconsin. Ultimately, they will be installed on CM-5 massively parallel supercomputers.
Results to Date: The development and evaluation of nonhydrostatic approaches to modeling the global oceans has been focused on with a view toward nested model systems that will explicitly simulate deep oceanic convection. This model incorporates artificial compressibility and semi-implicit differencing in a fashion similar to current atmospheric mesoscale models. When these techniques are used with multiple subcycling to separate the slow transport and other calculations, efficiencies comparable to current hydrostatic models have been achieved. The model development was performed on a Thinking Machines CM-5 parallel supercomputer, and we are now in the process of optimizing the code to make best use of the node vector processors.

We are now completing the computation of the spherical test suite proposed by Jakob, Hack, and Williamson and we will be evaluating the cost effectiveness of the local spectral approach with these results.

\section{DEVELOPMENT OF AN ADVANCED FINITE-DIFFERENCE ATMOSPHERIC GENERAL CIRCULATION MODEL}

\author{
ARAKAWA, A., and MECHOSO, C. R. \\ UNIVERSITY OF CALIFORNIA AT LOS \\ ANGELES
}

$\begin{array}{ll}\text { FY } 1993 & 173 \\ \text { FY 1992 } & 140 \\ \text { FY 1991 } & 121\end{array}$

$09 / 01 / 93-08 / 31 / 94$ 
Objective: To develop a higher-resolution finitedifference model with improved process parameterizations that is suitable for a massively parallel computing environment.

Product: Improved methods for describing physical processes in atmospheric and ocean GCMs and the development of these models on massively parallel computer systems.

Approach: A documented copy of the current model code will be provided to the CHAMMP Program, and help will be provided to CHAMMP researchers to install the code on parallel supercomputers. As improvements to the model are made, they will be transferred to the CHAMMP community.

Improved physical parameterizations will be developed for cloud pro resses, convection, land-surface exchange, and the planetary boundary layer. These efforts will be partially supported by other funding agencies, particularly NASA. These parameterizations will be based upon information from recent observational programs and theoretical advances.

The computational framework of the model will be improved. Recent developments in more-accurate numerical methods will be incorporated into the model so that it more faithfully simulates the real atmosphere. These improved formulations partially overcome the inaccuracies in finite-difference modeling that gave rise to the spectral models. Of particular importance is the improved representation of flow near the poles, which has been problematic in global finite-difference models that use a spherical coordinate system.

Sensitivity experiments will be run to test the improved parameterizations and methods as a function of model resolution. That models perform better at higher resolutions is well known; the process parameterizations are more faithful to the real world at finer spatial scales. These sensitivity tests will examine how well the parameterizations scale with different grid spacings.

Results to Date: Our efforts in developing model physics emphasize parameterizations of cumulus convection, cloud formulation, and orographic gravitywave processes. A revised version of the ArakawaSchubert cumulus parameterization, which includes the effect of rainwater drag both in updrafts and downdrafts, has been implemented into the UCLA AGCM, and its impact is being investigated. We are also evaluating a version of the AGCM with prognostic equations for ice and liquid water. Progress is being made in implementing a recently developed parameterization of orographic gravity-wave drag.

The vertical grid structure of the AGCM is being changed to eliminate vertical computational modes. In parallel, a global model is being developed that is based on a generalized vertical coordinate that can be nearly identical to the sigma-coordinate near the lower boundary and the standard theta-coordinate away from the boundary with a smooth transition between the two.

We have analyzed the simulated seasonal cycle in a multidecadal integration of the UCLA AGCM coupled to the Geophysical Fluid Dynamics Laboratory (GFDL) Modular Ocean Model (MOM). A version of this model was run distributed between a Cray Y-MP and an Intel Delta.

\section{AN EVALUATION OF THE APPROPRIATE LAND-SURFACE RESOLUTION FOR CLIMATE MODELS}

AVISSAR, RONI

\section{RUTGERS UNIVERSITY}

$\begin{array}{lr}\text { FY } 1993 & 99 \\ \text { FY } 1992 & 103 \\ \text { FY } 1991 & 0\end{array}$

08/01/93-07/31/94

Objective: To evaluate the resolution at which heterogeneities in land-surface heat- and water-flux distribution affect the large-scale atmospheric structure described by GCMs for climate studies.

Product: An analysis of the scale significance and sensitivity of GCMs to surface parameters used in GCM boundary-layer parameterizations.

Approach: A statistically based model of land-surface heterogeneity will be developed to describe subgridscale surface fluxes for integrated-grid-scale fluxes in GCMs. The model will be tested to examine the nonlinear sensitivities to the 13 parameters that must be specified. A novel differential sensitivity-analysis algorithm will be employed to reduce the total number of sensitivity runs required from several thousand to several hundred. A mesoscale model will be employed 
to explicitly describe on a fine mesh the parameterized heterogeneities in the subgrid model to test the scheme.

Results to Date: To evaluate which of the land-surface characteristics are of greatest importance for atmospheric models, the Fourier amplitude-sensitivity test (FAST) was applied to the land-atmosphere interactions dynamics (LAID), a land-surface scheme for atmospheric models based on the "big-leaf" approach. Various probability density functions (PDFs) of land-surface characteristics under a broad range of atmospheric conditions were assumed, and the analysis showed that the land-surface parameters that affect the energy fluxes $i$ the atmospheric surface layer (ASL) most are stomatal conductance and surface roughness in vegetated land and soil-surface wetness and surface roughness in bare land. Albedo and leaf-area index (where vegetation completely covers the ground surface) affect these fluxes as well, but to a lesser extent.

The impact of spatial variability of these five landsurface characteristics (i.e., stomatal conductance, soil surface wetness, surface roughness, leaf-area index, and albedo) on the energy fluxes in the ASL was investigated with a land-surface scheme based on a statisticaldynamical approach. All possible combinations of various PDFs of these five characteristics and a broad range of atmospheric conditions were considered.

A comparison of the ASL energy fluxes calculated with either the PDFs or the corresponding mean characteristics indicated that, under unstable atmospheric conditions, variations in stomatal conductance and leaf-area index have the most significant effect on spatially integrated energy fluxes from vegetated surfaces. With bare land, soil-surface wetness strongly affected the ASL energy fluxes. Under stable atmospheric conditions, surface roughness appeared to have the predominant effect on these fluxes.

\section{CHAMMP DETAILEE ASSIGNMENT}

BADER, DAVID C.

\section{PACIFIC NORTHWEST LABORATORY}

$\begin{array}{ll}\text { FY } 1993 & 225 \\ \text { FY } 1992 & 225 \\ \text { FY } 1991 & 225\end{array}$

$10 / 01 / 92-09 / 30 / 93$
Objective: To provide a scientist familiar with largescale computer modeling to direct and coordinate the CHAMMP climate-modeling program.

Product: A well coordinated CHAMMP Program.

Approach: An assignee to DOE's Environmental Sciences Division will provide technical input to the GCRP staff, review program plans and proposals and make recommendations regarding their disposal, aid in coordinating project planning and research activitics among CHAMMP investigators, and perform any other duties assigned that are consistent with detailee responsibilities and limitations established by DOE.

\section{EXPLOITATION OF PARALLELISM IN CLIMATE MODELS}

BAER, FERDINAND

\section{UNIVERSITY OF MARYLAND}

$\begin{array}{ll}\text { FY } 1993 & 279 \\ \text { FY } 1992 & 273 \\ \text { FY } 1991 & 215\end{array}$

09/01/93-08/31/94

Objective: To explore new methods of GCM structure and execution that exploit massively parallel computers.

Product: Improved methods for describing physical processes in atmospheric and ocean GCMs, algorithms to execute these models on massively parallel computer systems, and models for climate-prediction studies.

Approach: The investigators will explore new algorithms that allow the concurrent computation of several time levels in a GCM. These time-compression algorithms will require the models to be restructured so that communication is minimized. Current GCMs require all grid points in the model to be marched forward concurrently, a step requiring communication and synchronization among the parallel processors.

The investigators will develop local subgrid-scale models that will provide the time- and space-averaged statistics to a climate model. These subgrid models will have a resolution that is independent of that of the climate model to encompass the appropriate range of spatial and temporal scales that are important to simulate the physical processes. 
The investigators will use massively parallel architectures to concurrently execute multiple model simulations to form an ensemble set of climate realizations. The simulations will have different initial conditions and would produce enough realizations to constitute an ensemble climate.

Resuits to Date: Tests with a two-level baroclinic atmospheric model on a CM-5 indicated that, as the number of processors is increased, savings in CPU time increases more rapidly with the interaction-coefficient (IC) method. Another approach that used low-order equations of the barotropic vorticity equation (BVE) and a Taylor's-series solution showed that, for a variety of cases tested under real atmospheric conditions, some problems can be solved in one machine cycle (i.e., one step of the Taylor's series) to $170 \mathrm{~h}$.

The impact of subgrid-scale energy statistics on the larger nonlinearly active scales that used the BVE with and without forcing was that prediction of the large scales in the test model diverged from the reference model in 5 to 10 days of integration for all conditions, but input of forcing in the synoptic scales slowed the error growth. Subgrid parameterization via explicit modeling, by examining the scaling properties used in a simple two-level climate model with an adjustment parametcrization, indicated that the climatic means of the simulation differed when the adjustment was computed on an alias-free grid and the minimaltransform grid.

\section{STUDIES OF OCEAN PREDIC'TABILITY A'T DECADE-TO-CENTURY TIME SCALES WITH A GLOBAL OCEAN GENERAL CIRCULATION MODEL IN A PARALLEL-COMPUTING ENVIRONMENT}

BARNETT, 'T. P., and MILLER. A.

\section{SCRIPPS INS'TITUTION OF OCEANOGRAPHY}

$\begin{array}{lr}\text { FY 1993 } & 178 \\ \text { FY 1992 } & 182 \\ \text { FY 1991 } & 16\end{array}$

$09 / 01 / 93-08 / 31 / 94$

Objective: To install a state-of-the-science, stochastically forced ocean GCM on a massively parallel computer and to carry out $1000+$-year runs to explore the natural variability of the global ocean circulation.
Product: A quantification of the natural variability of the ocean component of the climate system.

Approach: The ocean GCM acquired from the Max Planck Institute (MPI) in Germany will be adapted to run on the Intel iPSC/860 parallel supercomputer at the San Diego Supercomputer Center (SDSC). This model has previously been run fully coupled to the MPI atmospheric GCM for a 100-year simulation. The code transfer to the SDSC parallel supercomputer will be carried out jointly between SDSC and Scripps Personnel. Optimization and adjustments in the model's coding will be conducted through the life of the project as experience on parallel computing platforms grows.

Two stochastic atmospheric-forcing models will be developed to form an interactive upper boundary for the ocean GCM. One model will be constructed from empirical orthoganal functions (EOFs) determined from global atmospheric-data sets. Noise resulting from shortterm weather events will be filtered out to isolate the longer-climate-scale driving modes. The data sets will then be reconstructed with a random stochastic component added to the atmospheric model to reproduce the natural atmospheric variability resulting from the integration of the short-term events. A second model will be similarly constructed from atmospheric-GCMderived data.

The differences in equilibrium states produced in the ocean GCM by the two atmospheric models will be examined. This study will determine the problems associated with coupling atmospheric and ocean GCMs, which currently tend to produce a climate that drifts away from the observed equilibrium state. The model will be run for 100-year simulations with each of the atmospheric forcing models.

Three 100-year model runs will be analyzed, the two just described and a third in which the model is coupled to a full atmospheric GCM at MPI. Analysis and intercomparison of the three runs will determine the time and space scales of natural variability, the mechanisms of model-climate drift, and preliminary estimates of the deterministic spatial and temporal limits of predictability of the ocean circulation.

Results to Date: Extensive study found that the isopycnal OGLM did not parallelize well on the Intel iPSC/860 because of the implicit numerical scheme used in the model. So we switched to a more conventional, but very fast, OGCM for the long ocean-model runs. 
The different atmospheric stochastic models were reconstructed and tested against atmospheric model and observation. They were (1) "white" in both space and time, and (2) "red" in space and "white" in time, and (3) "red" in both space and time. All three atmospheres were successfully coupled to the OGCM and run for thousands of years of simulated time. The results of these runs are now being analyzed.

\section{COMPARISON OF METHODS FOR SOLVING SPHERICAL SHALLOW-WATER EQUATIONS ON THE CONNECTION MACHINE}

\section{BAUMGARDNER, JOHN R.}

LOS ALAMOS NATIONAL LABORATORY

$\begin{array}{lr}\text { FY } 1993 & 150 \\ \text { FY } 1992 & 100 \\ \text { FY } 1991 & 0\end{array}$

$10 / 01 / 91-09 / 30 / 92$

Objective: To employ the shallow-water equations on the sphere to explore and refine the particle-in-cell (PIC) method as a viable alternative approach to existing formulations for GCM fluid dynamics.

Product: An accurate and robust PIC formulation of the shallow-water equations on the sphere that displays high efficiency on parallel computers and can readily be extended to three dimensions for real GCM application.

Approach: Various interpolation schemes, time integration methods, and particle bookkeeping procedures will be explored with a triangular meshing of the sphere to identify a specific formulation that provides high accuracy, high efficiency, and robustness. The search for a parallelizable time-implicit scheme will be given special priority.

Results to Date: Using the 2-D shallow-water equations as our development vebicle, we have demonstrated an implicit formulation on an almost uniform icosahedral mesh. The method uses, on the average, four particles per mesh point, linear interpolation functions between particles and mesh, and an extremely efficient multigrid solver for the implicit equations. To maintain data locality, the particle data is shifted to the nearest mesh point on each time step. The cost of the method is about 3000 floating-point operations per mesh point per time step, independent of resolution.
The Lagrangian PIC method of fers advantages of being positive definite while displaying exceptionally low numerical diffusion. We have also shown that it can be made fully implicit at low cost. We have written a parallel version of the multigrid solver that displays a communications overhead of $<10 \%$, and we are in the process of creating a message-passing version of the entire code that, we believe, will run at very high efficiency on a variety of parallel platforms. We foresee no major obstacles to extending the method to three dimensions for a full-ocean application.

\section{HIERARCHY OF THERMOHALINE CIRCULATION MODELS}

CESSI, PAOLA

UNIVERSITY OF CALIFORNIA AT SAN DIEGO

$\begin{array}{lr}\text { FY 1993 } & 85 \\ \text { FY 1992 } & 0 \\ \text { FY 1991 } & 0\end{array}$

09/01/93-08/31/94

Objective: To use a hicrarchy of simplified models to study the dynamics of the ocean thermohaline circulation and its effects on climate.

Product: A quantitative evaluation of the sensitivity of the thermohaline circulation to assumptions about surface forcing.

Approach: Three models of the north-south ocean thermohaline circulation will be used to study the dynamics and time-dependent behavior of the large-scale ocean flows that influence climate. The sensitivity of the circulation to changes in the latitudinal distribution of heat and freshwater flux will be studied. The simplest representation will be a diffusion model with two boxes crudely representing the equatorial and high-latitude oceans. Idealized convection simulations will then be employed to test the conclusions drawn from the boxmodel studies. A zonally averaged model will be developed that will include the latitudinal effects of the oceanic mass distribution. The use of the simplified models will provide direction for the development of full-ocean GCMs for climate research.

Results to Date: New project. 


\section{SCIENTIFIC DEVELOPMENT OF A MASSIVELY PARALLEL OCEAN CLIMATE MODEL}

CHERVIN, ROBERT M.

NATIONAL CENTER FOR ATMOSPHERIC RESEARCH

$\begin{array}{lr}\text { FY } 1993 & 102 \\ \text { FY } 1992 & 98 \\ \text { FY } 1991 & 98\end{array}$

$09 / 01 / 93-08 / 31 / 94$

Objective: To develop the Chervin-Semtner eddyresolving ocean GCM (OGCM) to run on massively parallel computers.

Product: State-of-the-science OGCMs that run on massively parallel computer architectures.

Approach: Research will be conducted on heat transport by the ocean circulation, the effects of nearsurface processes and their model parameterizations on long-term climate simulations, and model-resolution requirements for climate-modeling studies of the ocean circulation.

Results to Date: Several sensitivity experiments have been performed with wind stresses based on wind analyses from the European Centre for Medium Range Weather Forecasts for 1980 to 1989. These additional parallel-ocean-climate-model (POCM) decadal integrations feature forcing by an independent seasonalcycle wind-stress climatology, by a sequence of monthly mean wind stresses for 1980 to 1989 , and a sequence of daily wind stresses for this same time span. These experiments provide a measure of the sensitivity of the thermohaline circulation and its associated heat transport to uncertainties in atmospheric winds.

Preliminary results with the new climatological forcing include a more realistic Antarctic Circumpolar Current (ACC) and a stronger flow than before in the Kuroshio Extension region and in the deep western Pacific. Transport of heat and salt are very sensitive to the details of the winds. In particular, the wind-driven Deacon cell in the Southern Hemisphere is much stronger than in the control experiment and provides very realistic salt transport. In addition, eddy variability and eddy heat transport are enhanced in the ACC.
Furthermore, these experiments indicate the degree of nonlinearity in the ocean's response to different degrees of time variability in wind-stress forcing, a major issue in developing coupling strategies between atmosphere and ocean models.

\section{REGIONAL CLIMATES IN GCMS}

CRANE, ROBERT G.

\section{PENNSYLVANIA STATE UNIVERSITY}

$\begin{array}{lr}\text { FY } 1993 & 58 \\ \text { FY } 1992 & 0 \\ \text { FY } 1991 & 0\end{array}$

09/15/93-09/14/94

Objective: To develop a method linking regional climate change with GCM predictions.

Product: A systematic method to predict regional climate perturbations from GCM predictions of climate change.

Approach: Transfer functions relating surfacetemperature and precipitation fields to the global circulation will be derived from observations of regional meteorological parameters and analyses of global weather patterns. These functions will be independently tested with a GCM used for climate analysis and prediction.

Results to Date: New project.

\section{STUDIES IN REGIONAL-CLIMATE- SENSITIVITY PREDICTABILITY AND TEMPORAL RESPONSE}

CROWLEY, T. J., and NORTH, G. R.

APPLIED RESEARCH CORPORATION

$\begin{array}{lr}\text { FY } 1993 & 145 \\ \text { FY } 1992 & 0 \\ \text { FY 1991 } & 0\end{array}$

09/29/93-09/28/94

Objective: To use noise-forced energy-balance models (nf-EBMs) to study climate sensitivity to external forcing, regional climate response to increasing $\mathrm{CO}_{2}$ 
concentration, the nonlinear effects of seasonal albedo changes, and differences in regional climate predictability resulting from geography.

Product: Preliminary estimates of climate sensitivities through multiple long-term integrations.

Approach: Climate sensitivity will be examined with parameters from the nf-EBM. The relationships between climate sensitivity and parameters in the nf-EBMs will be determined by comparing correlations from the data with those from the model. Adjusting the parameters in the model to give the correct correlations will yield quantitative information about the relationships among these parameters in the real climate system.

Transport by the atmospheric and ocean general circulations will not be explicitly included in the nfEBM, but will be accounted for by horizontal diffusion. But the rate of diffusion will not be fast enough to overcome the land-ocean differences in heat capacity, thereby producing geography-dependent lag in the timing of the climate system's response to greenhouse warming. Coupling the nf-EBM with an ocean upwelling-diffusion model will crudely simulate the ocean's role in delaying potential climate warming.

The linear and nonlinear effects of the nf-EBM runs will be separated by statistically comparing a version with an interactive snow albedo coupled to a seasonal cycle to one without an interactive albedo. The use of the nonlinear nf-EBM in the above two studies will also be explored.

Sophisticated statistical methods will be used to determine the capacity of several nf-EBMs to perform decade-to-century climate predictions. These methods can provide useful techniques for subsequent analysis of long GCM runs.

Results to Date: We examined frequency-dependent theoretical orthogonal functions (TOFs) in a spectralfunction space of the noise-response field from an energy-balance model with a deep ocean. These frequency-dependent TOFs are an essential ingredient in formulating an optimal method for signal detection. Because of the length and the sampling bias, we cannot calculate these functions accurately from observational data. A comparison with empirical orthogonal functions of observations indicated that the model produces useful sets of orthogonal functions that can be used for developing an optimal detection or prediction method.
Using these TOFs, we attempted to develop and test an optimal detection method based on a variational principle in which the mean square error is minimized. The method is a spatio-temporal filter. The preliminary results of the method as applied to the greenhouse warming and sunspot problems are very encouraging. The optimal filter increases the signal-to-noise ratio of the greenhouse-warming signal greatly. Also, the feeble sunspot signal is enhanced tremendously.

\section{CHAMMP CLIMATE-SYSTEM MODELING}

DANNEVIK, W. P., and MIRIN, A. A.

\section{LAWRENCE LIVERMORE NATIONAL LABORATORY}

$\begin{array}{ll}\text { FY } 1993 & 500 \\ \text { FY } 1992 & 450 \\ \text { FY } 1991 & 146\end{array}$

$10 / 01 / 91-09 / 30 / 92$

Objective: To develop an advanced generation of finite-difference atmospheric and oceanic GCMs for MIMD-architecture (multiple-instruction, multiple-data) high-performance computers and to investigate computational issues associated with coupling atmospheric, oceanic, and land-surface models.

Product: Optimized finite-difference oceanic and atmospheric GCMs running on massively parallel systems and an optimized coupled oceanic/atmospheric/land-surface model running on such a platform.

Approach: The GFDL Modular Ocean Model and the UCLA Atmospheric General Circulation Model will be converted to massively parallel systems by decomposing the spatial domain into vertical columns having (logically) rectangular cross-section in latitude and longitude and by advancing the finite-difference solution concurrently on each of the subdomains. The fastest possible performance will be obtained on the target architectures while maintaining portable code to ensure transportability to as many MIMD architectures as possible. The codes will be modularized down to the individual process scale to the extent possible, and issues of scalability, load-balancing, and improvement of numerical-solution algorithms and techniques will be investigated. 
Results to Date: The completely parallelized AGCM (dynamics plus physics) has been validated on several platforms, most recently the CM-5 at LANL. Efficiency studies have been carried out on the CM-5 and the BBN-TC2000 and on SUN/IPC and IBM RS/6000 clusters; for the cluster computations a comparison of PVM and P4 has been carried out. An initial version of the OGCM has been parallelized on the BBN-TC2000, and preliminary efficiency studies have been undertaken. A driver to handle a colupled OGCM/AGCM/LSM has been constructed and successfully tested for an uncoupled OGCM/AGCM calculation.

\section{LAND COMPONENT OF THE GLOBAL CLIMATE SYSTEM WITH ADEQUATE SPATIAL RESOLUTION}

DICKINSON, ROBERT E.

\section{UNIVERSITY OF ARIZONA}

$\begin{array}{ll}\text { FY } 1993 & 129 \\ \text { FY } 1992 & 123 \\ \text { FY } 1991 & 117\end{array}$

09/01/93-08/31/94

Objective: To develop land-surface-process parameterizations for GCMs.

Product: Climate-model approaches to describing the land surface and an improved BATS parameterization.

Approach: The current BATS code will be recoded, restructured, and documented to make it conform to programming standards now ascribed to by the climatemodeling community. Experiments will be performed with the scheme to identify shortcomings and weaknesses. A version of this code will then be developed to conduct simulations at $0.5^{\circ}$ land-surface resolution that is independent of GCM-grid resolution. A subgrid-scale description will be developed from GCM grid-scale average precipitation, radiation, and cloudiness for use by the BATS parameterization. Sensitivity experiments will be conducted with BATS implemented within NCAR's CCM2, a state-of-thescience spectral-transform climate model, to test the scheme's ability to simulate climate anomalies. And simple visualization tools will be developed to enhance evaluation of fine-mesh output.
Results to Date: A numerical framework of a land submesh underlying each mesh point of a GCM was developed to compute the distributions of subgrid-scale precipitation, soil moisture, net radiation, soil properties, elevation, and vegetation cover.

The BATS and VBATS (vectorized BATS) codes have been coupled to CCM2 on the NCAR Cray Y-MP. VBATS, with longitudinal loops, has been available since July 1993 as a standard option for CCM2 users.

A soils data set has been developed for the land submesh starting with the Zobler version of the FAO soils database and using rules developed at the Goddard Institute for Space Studies (GISS) for relating soil classifications to soil physical properties. The GRASS software has been used for visualization, interpretation, and control of errors. Boundary data is prescribed at $0.5 x$, which can be related to an approximately 32 -point submesh under each grid point of the $T-42$ match.

Climatological records for hourly precipitation over the United States have been examined to establish monthly mean spatial patterns for the average frequency and intensities of precipitation, and statistical relationships between CCM2 parameters and intensity of precipitation have been developed. The parameterization provides an expected precipitation intensity based on $\mathrm{CCM}_{2}$ parameters at those points where $\mathrm{CCM} 2$ has produced precipitation. A prototype code to specify what fraction of the submesh is covered by precipitation has been developed, and initial test simulations have been carried out.

\section{PARALlEL ATMOSPHERIC CLIMATE MODEL}

DRAKE, JOHN B.

OAK RIDGE NATIONAL LABORATORY

$\begin{array}{ll}\text { FY 1993 } & 800 \\ \text { FY 1992 } & 835 \\ \text { FY 1991 } & 778\end{array}$

$10 / 01 / 92-09 / 30 / 93$

Objective: To provide a usable parallel implementation of the CCM2, to improve algorithms that couple atmospheric and other components of a climatesimulation system, to produce algorithm libraries and prototype software to aid in the development of parallel 
climate models, and to sponsor workshops for the climate modeling community.

Product: Advances in numerical methods and parallelprocessing techniques required to model the atmospheric component of the climate system on massively parallel computers.

Approach: An atmospheric-GCM-based climate model that uses the spectral-transform method (the NCAR CCM2) will be improved and developed for parallel climate simulations, complementing the Model Development Team's efforts to provide usable, optimized, production versions of the parallel CCM2.

New numerical methods for dynamics and novel parallel algorithms for climate-process computations will be tested as candidates for inclusion in the climate model with the nonlinear shallow-water equations on the sphere. A variety of numerical methods will be investigated for numerical accuracy and efficiency for dynamics calculations as well as parallel performance and suitability for massively parallel computation.

Portable parallel implementations of the CCM2 and follow-on models will be made available for use by the CHAMMP research community. Portability will be based on message passing and the use of tools like PICL and PVM. Software tools will be modified or developed as the need arises. Periodic workshops focused on this and related research will be organized.

Results to Date: The CCM2 code for the Cray Y-MP was recently completed at NCAR. A parallel, distributed-memory, message-passing implementation of the CCM2 is currently undergoing testing and validation. Parallelism is based on a latitudinal and longitudinal decomposition of the data structures. The message-passing version has been tested on several computing platforms, including the Intel iPSC/860, Intel Delta, Intel Paragon, Kendall Square KSRI, and IBM SP1 as well as a network of workstations. A dataparallel version of the CCM2 is being produced for the Thinking Machines CM-5 in conjunction with the message-passing implementation.

An experimental model based on the CCM2 and a fully semi-Lagrangian treatment of advective terms has been developed. Parallel spectral algorithms based on the ring pipeline, global vector sum, and various transpose procedures were studied for efficiency. The study was conducted in the context of the shallow water equations and their parallel solution on message-passing multicomputers. Efficient parallel fast Fourier transforms (FFTs) based on the Bluestein and other algorithms have been implemented in a message-passing version. The more general FFT will be incorporated in the parallel shallow-water-equation code and will be used in the parallelized CCM2 to remove the restriction that resolutions have a power of 2 number of longitudinal grid points.

ORNL hosted the third CHAMMP workshop on the numerical solution of partial differential equations in a spherical geometry.

\section{DEVELOPMENT OF AN ADVANCED REPRESENTATION OF CUMULUS CONVECTION}

\author{
EMANUEL, KERRY A. \\ MASSACHUSETTS INSTITUTE OF \\ TECHNOLOGY
}

$\begin{array}{ll}\text { FY } 1993 & 96 \\ \text { FY } 1992 & 91 \\ \text { FY } 1991 & 85\end{array}$

09/01/93-08/31/94

Objective: To develop a new model of cumulus transport within GCMs that uses a different hypothesis from the entraining cloud model now in widespread use.

Product: Cumulus convection parameterizations for atmospheric GCMs.

Approach: Recent observations will be used to develop a new model of cumulus transport within GCMs that uses a different hypothesis from the entraining-cloud model now in widespread use. It will, instead, adopt an episodic-mixing approach, in which parcels enter the cloud at cloud base, move vertically through the cloud in updrafts without mixing, then instantaneously mix when they reach the neutral-buoyancy level. Cloud microphysical parameters will determine the amount of cloud water converted to precipitation.

Results to Date: The classical semiprognostic test of cumulus parameterizations has been found to be deeply flawed and to not realistically assess the quality of convective schemes. We have therefore developed an optimal method for simultaneously optimizing the 
parameters of convective schemes and assessing their quality, using long time series of sounding-array measurements from the tropics. This technique involves using measurements of array-averaged vertical velocity, horizontal advections of temperature and water vapor, surface fluxes, and radiative transfer to integrate a 1-D model that includes tendencies predicted by the convective scheme in question. The most sensitive predictor, relative humidity, is used to optimize all the scheme's adjustable parameters. Progress is being inhibited to some extent by the lack of high-quality measurements of water vapor in the tropical upper troposphere, a very important quantity to predict in climate models because of its strong effect on infrared radiative transfer.

\section{ROLE OF BAROCLINIC-WAVE AMPLITUDE AND TRANSPORT VARIATION IN CLIMATE CHANGE}

FARRELL, BRIAN F.

\section{HARVARD UNIVERSITY}

$\begin{array}{lr}\text { FY } 1993 & 52 \\ \text { FY } 1992 & 0 \\ \text { FY } 1991 & 0\end{array}$

$08 / 15 / 93-08 / 14 / 94$

Objective: To determine the role of planetary and synoptic-scale baroclinic waves in climate change.

Product: A method for quantifying the effect of changes in climate forcing on planetary and synopticscale baroclinic waves.

Approach: A baroclinic zonal jet model will be examined with adjoint techniques that incorporate the theory of non-self-adjoint dynamical systems. Modifications in the planetary-scale heat and moisture transports resulting from changes in the distribution of baroclinic wave phase and amplitude can be determined from this approach. The forced response of the system to changes in the climate will be examined.

Results to Date: The adjoint analysis of the baroclinic wave model has been completed, and the sensitivity of heat and moisture transport to changes in the system has been determined.
PARALlel ATMOSPHERIC CLIMATE MODEL

FOSTER, I. T.

ARGONNE NATIONAL LABORATORY

$\begin{array}{ll}\text { FY } 1993 & 520 \\ \text { FY } 1992 & 520 \\ \text { FY } 1991 & 195\end{array}$

$10 / 01 / 92-09 / 30 / 93$

Objective: To provide the technological advances in numerical methods and parallel processing required to model the atmospheric component of the climate system on massively parallel computers.

Product: An advanced climate model with significantly greater capability than that of current models and parallel-processing techniques that can be applied to other such models.

Approach: Research will be conducted in three areas: advanced numerical methods, parallel model development, and parallel processing techniques. Parallel algorithms for existing numerical methods and new methods and algorithms suitable for large-scale parallel climate models will be developed and evaluated. Prototype implementations of methods and algorithms applied to the shallow-water equations on the sphere will be used to study accuracy, efficiency, and suitability for massively parallel execution.

The NCAR CCM2 will be investigated with the goal of producing a usable parallel implementation for distributed-memory MIMD computers. Parallel algorithms, promising new methods, and techniques for new model physics will be incorporated into the parallelized CCM2 (PCCM2) for further evaluation.

Parallel-software techniques and tools will be designed and developed to support algorithm development. Particular problems to be tackled will include load balancing and modular construction of large models.

Results to Date: PCCM2, incorporating parallel spectral transform and semi-Lagrangian transport algorithms, has been developed and is operational on the 512-processor Intel Delta and an IBM SP-1. Detailed validation studies show that the code is equivalent to CCM2. History-tape I/O, parallel checkpointing, and simple monthly 
averaging have been incorporated, allowing long-term simulations.

Specialized parallel-I/O and matrix-transpose algorithms have been developed for mesh-connected parallel computers, such as the Intel Delta. A portable parallel matrix-transpose library provides reproducability on different numbers of processors and permits the use of non-power-of-2 FFTs. A specialized version of the library minimizes network contention in mesh-based computers. The parallel-I/O library permits overlapping of computation with I/O operations.

PSTSWM, a parallel shallow-water-equation testbed code that is based on Hack and Jacob's STSWM code and that permits empirical and analytic evaluation of a wide range of algorithm altematives for parallel spectral models, has been developed. It is useful as an algorithm testbed, pedagogical tool, and performance benchmark.

Effective load-balancing algorithms have been developed for parallel climate models and these algorithms have been integrated into PCCM2.

\section{MODELING OF LAND-SURFACE-ATMOSPHERIC DYNAMICS FOR CHAMMP}

GUTOWSKI, WILLIAM J., JR.
IOWA STATE UNIVERSITY
$\begin{array}{cr}\text { FY } 1993 & 184 \\ \text { FY } 1992 & 170 \\ \text { FY } 1991 & 0\end{array}$

08/01/93-07/31/94

Objective: To accurately describe land-surface hydrological and vegetative effects in GCMs.

Product: A model parameterization that incorporates land-surface processes and feedbacks with the atmosphere.

Approach: An existing model of land-surface-atmosphere exchange and surface hydrology will be extended and will use a single-column version of an atmospheric GCM as the development testbed.

Results to Date: None reported.
IMPROVEMENT IN MOIST AND RADIATIVE PROCESSES IN HIGHLY PARALLEL ATMOSPHERIC GENERAL CIRCULATION MODELS: VALIDATION AND DEVELOPMENT

\author{
HACK, JAMES J.
}

\section{NATIONAL CENTER FOR ATMOSPHERIC RESEARCH}

$\begin{array}{lr}\text { FY } 1993 & 170 \\ \text { FY 1992 } & 164 \\ \text { FY 1991 } & 0\end{array}$

08/01/93-07/31/94

Objective: To develop state-of-the-science process parameterizations for use in atmospheric GCMs running on massively parallel computers.

Product: A model testbed to provide a convenient and efficient development environment to test the parameterizations, moist-convective process representations with proper scale selection for atmospheric GCMs, and atmospheric-radiative-transfer representations for GCMs.

Approach: As climate-model resolution increases, the process representations for convection and radiative transfer nust properly scale with the spatial grid sizes of the model. Additionally, current GCM-based climate models are unable to treat clouds and radiative transfer with enough accuracy to reduce the large uncertainty in recent climate-change simulations. The parameterizations for these processes will be improved with information available from recent field data and detailed model results. To provide a framework for rapid and efficient testing of these parameterizations, 1-D and 2-D reduced versions of GCMs will be built that will simulate the performance of the parameterizations within a GCM context.

Results to Date: None reported. 


\section{PARAMETERIZATIONS IN HIGH RESOLUTION ISOPYCNAL WIND-DRIVEN OCEAN MODELS}

\author{
JENSEN, T., and RANDALL, D. A. \\ COLORADO STATE UNIVERSITY
}

$\begin{array}{lr}\text { FY } 1993 & 56 \\ \text { FY } 1992 & 57 \\ \text { FY 1991 } & 0\end{array}$

$08 / 01 / 93-07 / 31 / 94$

Objective: To develop a high-resolution isopycnalcoordinate ocean-circulation model.

Product: A computer model that can be used to test the usefulness of the isopycnal-modeling approach for climate prediction.

Approach: Isopycnal-ocean-model formulations use spherical coordinates to describe the two horizontal dimensions that are parallel to the earth's surface and constant potential density surfaces (or isopycnics) as the vertical coordinate. In the absence of diabatic effects and mixing, ocean-fluid parcels will traverse along isopycnal surfaces. This property has advantages in inite-difference numerical forms of the equations of motion because artificial numerical mixing is reduced as the equations are integrated. Because isopycnal surfaces are not fixed in space, other problems associated with this coordinate system can offset its advantages. These issues will be explored to determine if isopycnal coordinate models are preferable alternatives to the models employing the standard vertical coordinate in global ocean modeling.

Results to Date: A single-layer version of the Indian Ocean model has been recoded for the CM-200 machine at LANL. The code makes very efficient use of CSHIFT operators to compute finite differences. It was used to run a model of the Indian Ocean with a resolution of $1 / 3^{\circ}$ by $1 / 3^{\circ}$ on the CM-200. Forced by annual and semiannual zonal winds in a $6^{\circ}$-wide band around the equator, 16 three-year runs were made. The runs were used to study a potential Kelvin-Rossby-mode resonance in the Indian Ocean when forced by semiannual zonal winds. Given the right combination of stratification, basin width, and periodicity of wind stress, equatorial Kelvin and Rossby waves combine to a standing mode with large amplitudes. The influence of the equatorial waves and associated jets on flows at poleward latitudes is found to be significant.

Programming of a new thermodynamic version of the model is well under way. It includes prognostic temperature and salinity for multiple layers in irregular geometries. The modular form of the code should ensure efficient code for both the Cray and the CM-5. The model includes simple mixed-layer physics and crossisopycnal mixing for deeper layers.

\section{MODELING OF HYDROLOGIC AND TRANSPORT PROCESSES IN RELATION TO CLIMATE CHANGE}

JOHNSON, DONALD R.

\section{UNIVERSITY OF WISCONSIN}

$\begin{array}{lr}\text { FY 1993 } & 111 \\ \text { FY } 1992 & 107 \\ \text { FY 1991 } & 0\end{array}$

$$
08 / 01 / 93-07 / 31 / 94
$$

Objective: To develop improved coordinate systems to describe atmospheric dynamics and transport in GCMs.

Product: An analysis of the accuracy of various coordinate system models used in atmospheric dynamics and transport.

Approach: Comparison simulations among various models with different coordinate systems will be run. The favorable accuracy obtained with isentropic coordinates has been known for some time, but the difficulty in describing the atmospheric boundary layer in models with an isentropic vertical coordinate has been a major obstacle. Recent progress in the development of boundary-layer parameterizations that result in more accurate descriptions of boundary-layer fluxes has made' isentropic modeling a feasible alternative to the pressure models.

Results to Date: Hydrologic and transport processes have been modeled in a series of experiments with versions of a hybrid isentropic-sigma $(\theta-\sigma)$ model, with approximately $85 \%$ of the atmosphere modeled with isentropic coordinates, and a standard sigma $(\sigma)$ model. The results shiow that the $\theta-\sigma$ model has substantial advantages over the $\sigma$ model in simulating transport processes important to climate studies. 
Comparisons have been made between simulations by global $\theta-\sigma$ and $\sigma$ models. The models were initialized with assimilated data, and idealized distributions of inert trace constituents were inserted into the atmospheres. The results show that the $\theta$ - $\sigma$ produces trace-constituent distributions with substantially greater integrity and that the $\sigma$ model distributions lack the intense gradients that should develop in baroclinic zones.

Experiments with channel versions of the two models, tested their capabilities to conserve four important meteorological quantities. The $\theta-\sigma$ conserves all four to a much higher degree of accuracy than does the $\sigma$ model.

\section{VARIABILITY AND PREDICTABILITY OF THE COUPLED OCEAN-ATMOSPHERE-LAND CLIMATE SYSTEM}

KINTER, JAMES L.

\section{INSTITUTE OF GLOBAL ENVIRONMENT AND SOCIETY, INC.}

$\begin{array}{lr}\text { FY } 1993 & 100 \\ \text { FY } 1992 & 0 \\ \text { FY } 1991 & 0 \\ & 09 / 30 / 93-09 / 29 / 94\end{array}$

Objective: To quantify model uncertainties and internal climate-model variability that result from GCM predictions.

Product: An analysis of the systematic errors and climate statistics produced by long GCM runs.

Approach: An existing, state-of-the-science GCM will be used to perform variability and uncertainty studies. Quantification of the internal atmospheric variability will be made by comparing long runs of a highresolution atmospheric GCM with a specified annual cycle of sea-surface temperature to long runs with the atmospheric model coupled to a dynamic ocean. The statistics of the short-term variability may differ between the two simulations. Multiple atmosphericmodel simulations will be conducted to examine the sensitivity of the predicted climate to resolution.

Results to Date: Numerical experiments were conducted at medium resolution to assess the sensitivity to the progression of the seasonal cycle of solar forcing.
By varying the length of the solar cycle forcing, we found that the tropical Pacific has a natural time scale for the development of the seasonal cycle. This means that when the solar forcing cycle is significantly shorter than this natural time scale, only a single annual peak in, say, surface-temperature distribution is found. This is coincident with the observation that the tropical Pacific has only a single temperature maximum even though the Sun crosses the equator twice a year. When the solar forcing cycle is longer than the ocean's natural time scale, a double peak associated with the two equatorial crossings by the Sun is found.

Because integrations of coupled models at low, medium, and high resolutions exhibit substantial climate drift, an anomaly-coupling technique was adopted to eliminate the drift. We found that the anomaly-coupled model produces a reasonable seasonal cycle in the equatorial oceans and includes better, although still weaker than observed, interannual variability.

We also began to assess and quantify the water-vaporfeedback effect in global climate change. The preliminary result is that about $50 \%$ of the climatic response to doubling $\mathrm{CO}_{2}$ is caused by the water-vapor feedback. Because of this high degree of sensitivity, this feedback must be accurately included in any coupledmodel simulation.

\section{COMPUTATIONAL SUPPORT FOR PARALLEL CLIMATE MODELING}

KLIEWER, KENNETH L.

\section{OAK RIDGE NATIONAL LABORATORY}

$\begin{array}{lr}\text { FY } 1993 & 600 \\ \text { FY } 1992 & 600 \\ \text { FY } 1991 & 0\end{array}$

$10 / 01 / 92-09 / 30 / 93$

Objective: To provide massively parallel computer resources and support for using these resources on the Intel Paragon parallel supercomputers.

Product: Atmospheric, ocean, and coupled climate models and codes running on the Intel Paragon message-passing, machines.

Approach: The Center for Computational Sciences at Oak Ridge National Laboratory will provide dedicated 
blocks of computer time to CHAMMP investigators. Center staff will assist CHAMMP researchers as model codes are developed and run on the Intel Paragon. Emphasis will be placed on support for operational considerations of the PCCM2 code on the Paragon, including compiler-optimization support, parallel-file system implementation for fast $1 / O$, and convenient scheduling and batch-submission procedures for development and model runs. Emphasis will also be placed on support of CHAMMP model-development teams implementing the POP ocean model, the UCLA AGCM, and the MOM model.

Results to Date: The 66-processor Intel Paragon is currently being used by CHAMMP model-development teams at ORNL, LANL, and LLNL. Atmospheric and oceanic GCMs have been ported to the Paragon and are being optimized and validated.

CHAMMP codes are being used in the acceptance test for the 512-processor Intel Paragon, and planned hardware upgrades are being considered in light of the I/O requirements of the GCMs.

\section{DYNAMIC HEAT AND MOISTURE TRANSPORT AND BAROCLINIC ADJUSTMENT}

LINDZEN, RICHARD S.

\section{MASSACHUSETTS INSTITUTE OF TECHNOLOGY}

$\begin{array}{lr}\text { FY } 1993 & 43 \\ \text { FY } 1992 & 0 \\ \text { FY } 1991 & 0\end{array}$

$$
08 / 15 / 93-08 / 14 / 94
$$

Objective: To develop a simple climate-assessment model that includes meridional distributions of temperature and other fields determined on the basis of baroclinic equilibration outside the tropics and Hadley circulations within the tropics and to interface these two major dynamic heat-transfer mechanisms.

Product: A simple, sophisticated climate model for determining the zonally averaged climate with offline evaluation of regional variations.

Approach: Nonlinear baroclinic equilibration will be examined both observationally and with numerical models to relate the cumulus environment to the mean tropical environment and to interface the Hadley regime and the baroclinic-eddy regime. The detailed properties of cumulonimbus convection are essential to the Hadley circulation and the mean thermodynamic properties of the tropics, including upper-level water vapor, which provides a crucial climate feedback.

A relatively simple mechanistic climate model will be developed that will incorporate the dynamic mechanisms together with the equations for radiative transfer to calculate the zonally averaged, seasonally varying climate. The implications of the new baroclinic state will be studied for planetary-scale stationary waves and their associated storm paths, critical elements in inferring regional climate features associated with global change.

Results to Date: New project.

\section{CHAMMP INITIATIVE ORGANIZATION}

MacCRACKEN, MICHAEL C.

\section{LAWRENCE LIVERMORE NATIONAL LABORATORY}

$\begin{array}{ll}\text { FY } 1993 & 225 \\ \text { FY } 1992 & 225 \\ \text { FY } 1991 & 225\end{array}$

$10 / 01 / 92-09 / 30 / 93$

Objective: To provide leadership of the CHAMMP Science Team, whose goal is to develop a new generation of climate models able to provide convincing estimates of the potential time-dependent, regional changes in climate that may result from the increasing atmospheric concentrations of greenhouse gases.

Product: Convening of the CHAMMP Science Team and a series of workshops that address improving parameterizations and numerical methods, applying advanced hardware and software, and improving understanding of climate predictability.

Approach: The CHAMMP scientific program will pursue a four-part strategy involving the use of advanced computer hardware, the development and application of advanced mathematical approaches, the use of improved representations of physical and chemical processes, and the investigation of the limits 
of climate predictability. The organization of this program will involve a widely based collaborative effort with a focused, mission-oriented objective. Gaining this objective will require outreach to and beyond the climate modeling community and to and beyond the several groups involved in climate modeling.

To accomplish this objective, the community of researchers will be assembled to study the challenges to be faced and to produce versions of existing models that perform well in the massively parallel environment. During this phase, improved understanding of the requirements for the next generation of models will be worked out.

Initial versions of advanced models will be constructed, tested, and used to investigate questions of climate predictability. This will require the ideas and contributions of many groups.

The initial versions of the advanced models will then be extended in their physics, and useful model simulations will be conducted.

Results to Date: The second CHAMMP Science Team meeting was held in Monterey, California, in March 1993 with representatives of the approximately two dozen CHAMMP projects, and it was held jointly with the Model Development Team participants. Significant progress was reported in the transfer of models to massively parallel computers, in the development of refined parameterizations and alternative numerical methods for use in such models, and in investigations of the limits of climate predictability. A compilation of extended abstracts and a meeting summary were prepared and distributed.

Several Science Team and related workshops were held during the year, including ones on partial differential equations on a sphere, ocean predictability, and alternatives to GCMs. The Alternatives to GCMs workshop concluded that, while no fully satisfactory alternatives exist, complementary approaches that could be helpful are available. An upcoming joint workshop with the Climate System Modeling Project on interdecadal variability (CSMP) was planned.

\section{MANAGEMENT OF THE MODEL- DEVELOPMENT COMPONENT OF THE CHAMMP PROGRAM}

\author{
MALONE, ROBERT C.
}

LOS ALAMOS NATIONAL LABORATORY

$\begin{array}{ll}\text { FY } 1993 & 950 \\ \text { FY } 1992 & 735 \\ \text { FY } 1991 & 150\end{array}$

$10 / 01 / 91-09 / 30 / 92$

Objective: To provide technical leadership of the model-development component of the CHAMMP Program, technical consulting on the use of the Los Alamos Connection Machine (CM-5), and CHAMMP model development at Los Alamos.

Product: A research plan for the model-development component of CHAMMP, technical consulting, and climate models that run on the CM-5.

Approach: With DOE management, the scope, objectives, and timetable for CHAMMP will be defined; the tasks to be pursued within the model-development component will be defined; and well-qualified personnel will be identified within DOE laboratories, universities, and other institutions to carry out those tasks. A person experienced in the development of large codes on the CM-5 will be hired. Appropriate models of the ocean and atmosphere will be implemented on the CM-5.

Results to Date: A program plan has been formulated and implemented. Four DOE laboratories (Argonne, Lawrence Livermore, Los Alamos, and Oak Ridge) and the NCAR are carrying out model-development work that encompasses spectral-transform and finite-difference atmospheric models and Eulerian (Bryan-Cox-Semtner) and isopycnal ocean models.

A technical staff member with extensive experience on the CM-5 has been hired to consult with CHAMMP researchers.

Two ocean models are now operational on the CM-5. An improved Bryan-Cox-Semtner model has been developed. The barotropic mode was reformulated in terms of surface pressure instead of stream function, the rigid-lid boundary condition was replaced with an implicit free-surface treatment, and an efficient parallel 
elliptic-equation solver was developed. A basin-scale isopycnal model developed by Rainer Bleck (U. Miami) is also operational on the CM-5. Research continues on 3-D elliptic-equation-solver techniques applicable to the fully implicit time-integration techniques used in Josef Oberhuber's global isopycnal-ocean model.

\section{FORWARD-IN-TIME DIFFERENCING FOR GLOBAL CIRCULATION MODELS OF OCEAN AND ATMOSPHERE}

MARGOLIN, LEN G.

\section{LOS ALAMOS NATIONAL LABORATORY}

$\begin{array}{lr}\text { FY } 1993 & 90 \\ \text { FY 1992 } & 0 \\ \text { FY 1991 } & 0\end{array}$

07/01/93-09/30/92

Objective: To develop a dynamical framework for global atmospheric- and ocean-circulation models based on modern forward-in-time differencing schemes.

Product: Prototype numerical models for climate prediction.

Approach: New model formulations employing Lax-Wendroff computational techniques will be developed and tested. These modern methods will substantially reduce the numerical diffusion associated with older forward-in-time approaches. They will have advantages over conventional leap-frog schemes because the solutions will not contain a computational mode. These systems of equations will map well onto massively parallel computer architectures because interprocessor communication is local.

Results to Date: A shallow-water-equation model based on forward-in-time methods has been constructed to describe the flow of a single-layer fluid over a rotating sphere. This model has been exercised for the standard set of test problems proposed by the NCAR-ANL-ORNL model group to evaluate model dynamics. Results from these tests indicate that forward-in-time difference methods, when coupled with semi-Lagrangian spatial differencing, produce more accurate solutions for long integration times than do the standard leap-frog methods coupled with standard second- and fourth-order differencing.

\section{PREDICTABILITY IN NUMERICAL MODELING OF OCEAN DYNAMICS}

MARGOLIN, LEN G.

LOS ALAMOS NATIONAL LABORATORY

$\begin{array}{lr}\text { FY 1993 } & 250 \\ \text { FY 1992 } & 170 \\ \text { FY 1991 } & 0\end{array}$

$10 / 01 / 92-09 / 30 / 93$

Objective: To apply nonlinear dynamical modeling methods to assess predictability and accuracy in computer models of the global ocean circulation.

Product: An analysis of the predictive potential found in ocean-circulation models.

Approach: Approximate-inertial-manifold (AIM) methods, which improve the accuracy of numerical simulations of dynamical systems and construct consistent representations of subgrid-scale processes, will be used to develop a subgrid-scale parameterization for modeling ocean dynamics. The underlying idea of the AIM is to represent the effects of the unresolved scales of motion (i.e., the high modes) in terms of the resolved or low modes. The error estimate that is derived from the construction of the parameterization will be used to estimate the predictive skill of the full ocean model.

Results to Date: Our technique for equations in multiple dimensions and with irregular boundaries has been generalized. A consistent treatment has been developed for compensating the error of both the spatial and the temporal discretization. On the more theoretical side, a scale analysis has been used to justify our closure procedure and to define the parameter regime in which it is valid. This analysis naturally contains a small parameter, which is the ratio of the mesh size to the problem size, that enables us to construct the AIM with regular perturbation techniques.

A computational study has been completed of the efficiency of the AIM. Our investigations suggest that the governing factor in estimating the improvement caused by AIM is the smoothness of the solutions, which in turn depends mainly on the smoothness of the forcing. The smoother the solution, the less improvement one sees with AIM. We have derived 
estimates of the improvement of the rate of convergence as a function of smoothness and verified them computationally. We have also shown that these estimates are nearly optimal.

\section{CRAY COMPUTER}

\section{MCCURDY, WILLIAM \\ LAWRENCE LIVERMORE NATIONAL LABORATORY}

$\begin{array}{ll}\text { FY } 1993 & 1000 \\ \text { FY } 1992 & 2000 \\ \text { FY } 1991 & 2800\end{array}$

$10 / 01 / 91-09 / 30 / 92$

Objective: To provide the climate modeling community with a substantial amount of computing resources (14,000 Cray-1 equivalent hours) to conduct long climate-model simulations.

Product: Long-model-run results for global-change studies and identification of model properties and characteristics in long-term simulations.

Approach: Large blocks of time were granted to climate researchers to perform long climate-model simulations of decade-to-century scale climate change. Approximately half of the resources were dedicated to participants in the World Climate Research Program's Atmospheric Model Intercomparison Project.

\section{OCEAN-CONVECTION PARAMETERIZATION}

$$
\text { PALUSZKIEWICZ, THERESA }
$$

\section{PACIFIC NORTHWEST LABORATORY}

$\begin{array}{lr}\text { FY } 1993 & 200 \\ \text { FY } 1992 & 190 \\ \text { FY } 1991 & 0\end{array}$

$$
10 / 01 / 92-09 / 30 / 93
$$

Objective: To develop parameterizations for penetrative and nonpenetrative convection in ocean GCMs.

Product: Convective parameterizations incorporated into an ocean GCM to increase the accuracy of deep-water- formation processes and to give increased prediction accuracy for ocean-atmosphere-coupled models.

Approach: A set of parameterizations for mixed-layer dynamics, sea-ice formation, and penetrative-convective mixing will be assembled and tested. The parameterizations will first be tested in a 1-D model. These 1-D experiments will determine the effectiveness of parameterizations and will help determine appropriate parameters, such as the entrainment rates and convective time scales. The parameterizations will then be incorporated into a modified version of the SemtnerChervin parallel ocean-climate model (POCM). The ocean GCM experiments will use an idealized domain representing the Greenland, Norwegian, and Iceland seas. Idealized and observed surface-forcing conditions will be used, and the relative performance of the parameterizations in a model ocean basin will be evaluated.

Results to Date: A 1-D penetrative-plume model has been constructed to parameterize the processes of shallow and deep convection in ocean GCMs (OGCMs). This model applies concepts similar to those used in atmospheric cumulus-convection parameterizations. The 1-D plume model simulates deep, penetrative (thermobaric) convection as well as shallow convection (mixed-layer deepening) when appropriate. A supporting entrainment-rate model was derived to take into account the effects of distributed sources of buoyancy and compressibility effects on the entrainment associated with convective plumes; the inclusion of this submodel allows the parameterization to use the appropriate entrainment rate based on the buoyancy profile produced by the OGCM. The 1-D penetrative-plume model is being evaluated against oceanographic data sets, the results of 3-D process models of penetrative plumes, and other mixed-layer deepening parameterizations.

Work began on simulations of the preconditioning in the Greenland Sea with the Semtner-Chervin OGCM. This research is in preparation for the incorporation of the parameterization into the OGCM. Sensitivity tests to OGCM resolution, wind-forcing, and cooling were conducted. Incorporation of the parameterization into the OGCM is planned for the fall and winter of 1993. 


\section{COUPLED ICE-OCEAN MODELS FOR CLIMATE RESEARCH}

\author{
PIACSEK, S. A., and PRELLER, R. H.
}

NAVAL RESEARCH LABORATORY

$\begin{array}{lr}\text { FY } 1993 & 192 \\ \text { FY } 1992 & 0 \\ \text { FY } 1991 & 0\end{array}$

09/30/93-09/29/94

Objective: To develop a state-of-the-science iceparameterization code suitable for climate studies.

Product: An efficient and accurate sea-ice model for inclusion in ocean models used for climate prediction.

Approach: The ice model for climate studies will be based on the Flato-Hibler parameterization and will make use of modern programming techniques for implementation on parallel computers. The model will be tested against both data and simulations from a more sophisticated dynamical ice model to ascertain its climate characteristics. Ultimately, the model will be included in versions of the Cox-Bryan finite-difference ocean model developed at the Geophysical Fluid Dynamics Laboratory.

Results to Date: New project.

\section{DEVELOPMENT OF AN ADVANCED FINITE-DIFFERENCE ATMOSPHERIC GENERAL CIRCULATION MODEL}

RANDALL, DAVID A.

\section{COLORADO STATE UNIVERSITY}

$\begin{array}{lr}\text { FY 1993 } & 156 \\ \text { FY 1992 } & 89 \\ \text { FY 1991 } & 83\end{array}$

09/01/93-08/31/94

Objective: To develop a higher-resolution finitedifference model with improved process parameterizations that is suitable for a massively parallel computing environment.
Product: Improved methods for describing physical processes in atmospheric and ocean GCMs and the development of these models on massively parallel computer systems.

Approach: A documented copy of the current model code will be provided to the CHAMMP Program, and help will be provided to CHAMMP researchers to install the code on parallel supercomputers. As improvements to the model are made, they will be transferred to the CHAMMP community.

Improved physical parameterizations will be developed for cloud processes, convection, land-surface exchange, and the planetary boundary layer. These efforts will be partially supported by other funding agencies, particularly NASA. These parameterizations will be based upon information from recent observational programs and theoretical advances.

The computational framework of the model will be improved. Recent developments in more-accurate numerical methods will be incorporated into the model so that it more faithfully simulates the real atmosphere. These improved formulations partially overcome the inaccuracies in finite-difference modeling that gave rise to the spectral models. Of particular importance is the improved representation of flow near the poles, which has been problematic in global finite-difference models that use a spherical coordinate system.

Sensitivity experiments will be run to test the improved parameterizations and methods as a function of model resolution. That models perform better at higher resolutions is well known; the process parameterizations are more faithful to the real world at finer spatial scales. These sensitivity tests will examine how well the parameterizations scale with different grid spacings.

Results to Date: The following parameterizations have now been successfully tested in the GCM: the new cumulus parameterization, which has been computationally optimized; the new cloud-microphysics parameterization, which has now been coupled to the GCM's radiation parameterization; and the new land-surface parameterization, including a partial representation of the atmospheric carbon budget. The results of all three new parameterizations are very encouraging. In addition, a new finite-difference scheme based on a spherical geodesic grid has been successfully demonstrated in a shallow-water model. The dynamics code of the GCM has been ported to the CM-5 at Los 
Alamos, but does not yet execute correctly. This problem is being debugged.

\section{SYMMETRIC TRUNCATION OF' THE SHALLOW-WATER EQUATIONS}

ROUHI, SAFIALI

\section{UNIVERSITY OF CALIFORNIA AT SAN DIEGO}

$\begin{array}{ll}\text { FY } 1993 & 122 \\ \text { FY } 1992 & 112 \\ \text { FY } 1991 & 105\end{array}$

$09 / 01 / 93-08 / 31 / 94$

Objective: To development a novel numerical framework to model geophysical fluid dynamics.

Product: The development of alternative numerical methods for GCMs of the atmosphere and ocean that can be executed on massively parallel computers.

Approach: Symmetric truncation will be used to develop a model system in which the number of conserved properties will increase as the degrees of freedom in the model increase. This system will be used to explore the relationship between conserved properties and accuracy of the model.

Reduction will then be used to cast the model in an Eulerian framework that will ultimately be required in a full climate model. A systematic examination of accuracy and conservation will define how the Eulerian model will be constructed. Finally, the shallow-water model will be extended to continuously stratified flow, which is more realistic for the atmosphere and ocean.

\section{CLIMATE-SYSTEM MODELING PROGRAM}

SCHIMEL, DAVID S.

\section{UNIVERSITY CORPORATION FOR ATMOSPHERIC RESEARCH}
FY 1993
150
FY 1992
150
FY 1991
0

$09 / 30 / 93-09 / 29 / 94$
Objective: To study interdecadal climate variability to define the limits of climate predictability.

Product: A series of workshops and postdoctoral research fellowships that will ascertain and define the limits and variability of interdecadal climate fluctuations.

Approach: The Climate Systems Modeling Program (CSMP) is a unique program sponsored by the University Corporation for Atmospheric Research that will draw upon intellectual resources throughout the academic and government scientific communities to advance the science of climate modeling. One effort of CSMP will addresse the problems of interdecadal climate variability, the time scale of most interest to DOE.

Results to Date: The Regional-Global Interactions (RGI) project is developing an ensemble of mesoscale simulations with a variety of horizontal boundary conditions and factorial combinations of land surface topography, antecedent soil moisture, and vegetation cover. This project will result in a database of both mesoscale responses and effects that originate from variability within the domain but that propagate to scales likely to influence the larger cir zulation and hydrology. A series of one-way coupled simulations is being developed that examine the effects of climate changes on ecosystem and land-surface hydrology properties.

The Interdecadal Variability project arranged a major workshop on the thermohaline circulation as a source of interdecadal variability. The meeting is intended to review the state of the science and to develop a plan for collaborative studies within the CSMP framework. The project has also been working on a zonal carbon-cycle model for studying climate- $\mathrm{CO}_{2}$ interactions on longterm $\mathrm{CO}_{2}$ exchange.

The Science of Assessment Project held a large and successful workshop. Follow-on from that workshop has involved significant input to the CEES on linking science to assessment in terms of products and timescales. In addition, a database is being developed of simple models used in policy formulation and gaming. Also, several CSMP Science Team members have become involved in the next round of IPCC and have been having extensive discussions with the agencies concerning a coordinated U.S. scientific contribution. 


\section{SCIENTIFIC DEVELOPMENT OF A MASSIVELY PARALLEL OCEAN CLIMATE MODEL.}

SEMTNER, ALBERT J.

NAVAL POSTGRADUATE SCHOOL

$\begin{array}{lr}\text { FY } 1993 & 150 \\ \text { FY } 1992 & 160 \\ \text { FY } 1991 & 87\end{array}$

09/01/93-08/31/94

Objective: To develop the Semtner-Chervin eddyresolving ocean GCM (OGCM) to run on massively parallel supercomputers.

Product: State-of-the-science OGCMs that run on massively parallel computers.

Approach: Research will be conducted on heat transport by the ocean circulation, the effects of nearsurface processes and their model parameterizations on long-term climate simulations, and model-resolution requirements for climate-modeling studies of the ocean circulation.

Results to Date: The magnitude and variability of oceanic heat transport have been examined in three 10-year global $1 / 2^{\circ}$ simulations with observed (European-Centre) winds. The simulations used climatological monthly, individual monthly, and actual daily winds of the period 1980 to 1989 . The results to date show major variations in heat transport from year to year and also as a result of different frequencies of sampling. A $1 / 4^{\circ}$ global model with a free surface and very realistic geometry has been built and run on the NCAR Cray Y-MP/8 with the observed monthly winds of 1985 to 1989. The initial fields were interpolated from the $1 / 2^{\circ}$ model. Within a few years, very significant improvements in mean and eddy characteristics have resulted. Topography and winds for a $1 / 6^{\circ}$ global grid were constructed, and results from the NCAR $1 / 4^{\circ}$ simulation were interpolated onto the $1 / 6^{\circ}$. grid to initialize a new simulation on the 1024-node CM-5 at Los Alamos.

\section{MASSIVELY PARALLEL GLOBAL OCEAN MODELING}

SMITH, RICHARD D.

\section{LOS ALAMOS NATIONAL LABORATORY}

$\begin{array}{lr}\text { FY 1993 } & 211 \\ \text { FY 1992 } & 200 \\ \text { FY 1991 } & 0\end{array}$

$10 / 01 / 92-09 / 30 / 93$

Objective: To develop a data-parallel version of the Semtner-Chervin eddy-resolving global ocean model for massively parallel supercomputers.

Product: An ocean-circulation model that runs efficiently on massively parallel supercomputers.

Approach: An existing state-of-the-science global ocean model will be ported to the CM-2 massively-parallel supercomputer and then installed on the 2048-processor CM-5 supercomputer, which is a factor of 100 more powerful. The model will be restructured, and several algorithmic changes will be made to increase its efficiency and capability. The model will be installed on the CM-5 as soon as the machine is available, and diagnostic runs will be made.

Results to Date: Development of a 3-D global ocean model for parallel architectures, based on the widely-used Bryan-Cox-Semuner ocean model, has continued. An implicit free-surface formulation has been developed and implemented for the barotropic (vertically integrated) flow. This technique is a natural extension of our previous rigid-lid surface-pressure formulation, but it is computationally much more efficient and physically more accurate.

The model has been extended to allow use of arbitrary orthogonal curvilinear coordinates on the sphere. This modification will allow basin-scale simulations with enhanced regional resolution and global simulations that include the Arctic region without high-latitude filtering.

A modular and portable version of the model that is designed to run on essentially any architecture was developed. It is currently running on a variety of machines, including the CM5, Intel Delta and Paragon, Cray Y-MP and C-90, and an IBM workstation cluster (with PVM). 
A decade-long integration at an average resolution of $1 / 6^{\circ}$ is currently being performed. This is, to date, the highest-resolution global ocean simulation ever performed.

\section{DYNAMICAL STRUCTURE OF CLIMATE SYSTEMS}

\author{
STEWART, H. BRUCE
}

BROOKHAVEN NATIONAL LABORATORY

$\begin{array}{ll}\text { FY } 1993 & 150 \\ \text { FY } 1992 & 150 \\ \text { FY } 1991 & 195\end{array}$

$10 / 01 / 91-09 / 30 / 92$

Objective: To investigate how dynamical-system approaches can contribute to an understanding of the internal variability of elimate on time scales of decades to centuries and whether they can help distinguish between internal fluctuations and changes induced by people.

Product: Dynamical-system techniques suitable for application to paleoclimate proxy data and interactive graphical software for analyzing multichannel data sets.

Approach: Multichannel-data methods will be developed, which will involve developing interactive graphic software that will allow the user to produce and analyze test embeddings with freely chosen subsets from multichannel data sets. The aim will be to determine which embedding demonstrates dynamical structure with forecastability.

Numeric exploration of the low-order, 27-variable, moist-atmospheric-circulation model of Lorenz using attractor-basin phase-portrait interpretations has produced an understanding of the behavior and forecastability of the model. Simulated data sets produced by the Lorenz model will be used as benchmarks for the interactive graphical software. The software will also be applied to selected paleoclimate proxy data.

Results to Date: The software has been developed to allow the user to select a subset of channels from a multichannel data set and to display phase-space trajectories in projected view with real-time 3-D rotation. Along the phase view, a recurrence plot of the same data is drawn. Coordinated viewing permits the user to highlight simultaneously a feature of the recurrence plot and of the trajectory. This coordinated viewing greatly facilitates interpretation of the recurrence plot and has proven very effective in tests with well-known, simple, chaotic attractors, such as the Roessler band.

The software is being benchmarked with simulated data produced by the low-order, 27-variable, moistatmospheric-circulation model of Lorenz. Application to selected paleoclimate proxy data, including tree rings and ice, cores, is under way.

\section{COUPLED-ATMOSPHERIC-OCEAN MODELS}

\author{
STONE, PETER H., and MAROTZKE, J.
}

\section{MASSACHUSETTS INSTITUTE OF TECHNOLOGY}

$\begin{array}{lr}\text { FY } 1993 & 49 \\ \text { FY } 1992 & 0 \\ \text { FY } 1991 & 0\end{array}$

$08 / 15 / 93-08 / 14 / 94$

Objective: To improve our understanding of atmosphere-ocean feedbacks, of the factors that control ocean heat transports, and of how these processes affect climate sensitivity.

Product: A coupled version of the GISS 2-D zonally averaged statistical-dynamical model of the atmosphere and the Marotzke-Willebrand 3-D ocean-circulation model.

Approach: A series of coupled-atmosphere-oceanpro ess models of increasing sophistication will be developed by systematically adding processes to earlier models until the ultimate coupled product is obtained. At each step, the process models will be used to assess the importance of the added processes in modeling climate and climate sensitivity.

Results to Date: New project. 


\section{PARALLEL METHODS FOR CLIMATE MODELING}

SWISSHELM, JULIE

SANDIA NATIONAL LABORATORIES

$\begin{array}{lr}\text { FY } 1993 & 50 \\ \text { FY } 1992 & 50 \\ \text { FY } 1991 & 0\end{array}$

10/0̣1/92-09/30/93

Objective: To develop fast and efficient algorithms for the solution of geophysical-fluid-dynamics problems on massively parallel computers.

Product: Recommendations for implementation of successful techniques in global ocean and atmospheric GCMs.

Approach: Methods from computational aerodynamics, such as multigrid and alternative time-marching algorithms, will be applied to appropriate test problems for the shallow-water equations, and successful techniques will be extended to a global ocean model. Algorithms will be optimized for use on massively parallel MIMD computers.

Results to Date: A finite-difference shallow-waterequation (SWE) solv $c_{i}$ lias been developed. To apply an $\mathrm{N}$-like multigrid scheme to the solution of the shallowwater equations, a reformulation of the equations in weak-conservation-law form has been derived and is the basis for the solver. Treatment of the inhomogeneous terms that arise in this formulation remains an issue to be examined with the implementation of a multigrid scheme.

The SWE solver is being used to test a number of schemes, including multigrid; residual smoothing; and alternative time-marching algorithms, such as multistage Runge-Kutta. Several test cases, from a suite developed by Williamson and colleagues, that are suitable for determining the validity and advantages of these schemes for accelerating the spin-up phase (from initial conditions to some equilibrium state) are used. Using convergence acceleration techniques is expected to significantly shorten the long spin-up times necessary for global ocean models to reach an equilibrium state, and additional test cases that will exercise this characteristic are being devised.

\section{CHAMMP CM-5 SUPPORT}

WHITE, ANDREW B.

LOS ALAMOS NATIONAL LABORATORY

$\begin{array}{lr}\text { FY 1993 } & 1200 \\ \text { FY 1992 } & 1200 \\ \text { FY 1991 } & 0\end{array}$

07/01/92-09/30/92

Objective: To provide access and resources for CHAMMP investigators on the massively parallel CM-5 supercomputer at the Los Alamos High Performance Computing Research Center.

Product: Atmospheric, ocean, and coupled climate models and codes running on the CM-5 supercomputer.

Approach: CHAMMP Investigators will be allotted approximately $25 \%$ of CM-5 machine resources.

\section{ATMOSPHERIC CHEMISTRY AND CLIMATE PREDICTABILITY: TOWARDS AN ADVANCED CLIMATE MODEL}

WUEBBLES, D. J., and PENNER, J. E.

\section{LAWRENCE LIVERMORE NATIONAL LABORATORY}

$\begin{array}{ll}\text { FY } 1993 & 300 \\ \text { FY 1992 } & 300 \\ \text { FY } 1991 & 282\end{array}$

$10 / 01 / 92-09 / 30 / 93$

Objective: To investigate the role of atmospheric chemistry in climate predictability and to develop the 3-D chemical-transport modeling capability needed by an advanced climate model.

Product: Understanding of the importance of atmospheric chemistry in climate predictions and a highly advanced, modeling capability for the investigation of energy and environmental issues.

Approach: The potential for atmospheric chemistry processes to improve the predictability of climate change will be examined. The possible range of chemical-transport processes than influence climate will 
be determined. Existing 2-D and 3-D atmospheric chemistry models will be used to evaluate the advantages of various types of modeling approaches (i.e., Eulerian or Lagrangian). The range of possible chemistry solvers of both stiff and nonstiff algorithms as applied to massively parallel computers will be investigated. A next-generation 3-D chemical-transport model will be designed and implemented on a massively parallel computer, and the model will be validated by comparison to available databases, such as those becoming available from satellite observations of atmospheric trace constituents.

Results to Date: The depletion of stratospheric ozone and the increase in tropospheric methane have been found to have strong effects in the radiation balance of the atmosphere and hence on climate. Also, the strong effects on climate caused by stratospheric and tropospheric aerosols from natural and anthropogenic sources along with the heterogeneous chemistry that takes place on these aerosols have been found to cause changes in the radiative forcings of similar magnitudes to that caused by $\mathrm{CO}_{2}$ buildup. Aerosols have also been found to cause substantial depletion in stratospheric ozone in the mid-latitude range.

A tracer-transport model that would run interactively with a working atmospheric GCM on massively parallel computers was developed and implemented on a parallel computer to serve as a base for future chemical transport modeling. It is based on an upwind van Leer advection scheme that is second order in space and capable of accurately following strong gradients. In addition to running interactively with the GCM, it can also run with known data-assimilated wind fields and known GCM winds. In the development, we followed the guidelines used to produce the LLNL parallel GCM. The paradigm used is that of 2-D domain, whereby the atmosphere is decomposed into patches and information is distributed through message passing. The key to this method is that columns remain complete in the vertical direction, thus allowing ease of radiation, photolysis, and microphysics calculations. Macros were also implemented that allow the same code to be run on a host of massively parallel computers, Cray computers, and networks of smaller UNIX workstations.

To study and compare the advantages and disadvantages of Lagrangian and Eulerian transport approaches and schemes, a Eulerian advection scheme was implemented into a 3-D Lagrangian tropospheric model. With all physics turned off, classic transport tests were done, including solid-body rotation and flow over the poles. Initial tests indicated the strong need for higher-order methods in the advection. First-order methods gave relatively large diffusion errors caused by the upstream differencing. Second-order gave good results. Fourthorder was somewhat better, but going to a higher order did not produce increasingly better results. In tests of pure advection, such as these, Lagrangian methods are nearly perfect. Thus, these comparisons would lead one to choose Lagrangian-based schemes. On the other hand, moving to Eulerian methods now provides the capability of resolving the stratosphere, which the Lagrangian scheme was unable to do. Furthermore, it is believed that the Eulerian scheme would be implemented on a parallel machine in a much more efficient fashion compared with the Lagrangian scheme.

To study the parallelization of chemistry solvers, a predictor-corrector solver was implemented on the LLNL parallel machine within the existing Lagrangian tropospheric chemical-transport model. Because chemistry solutions require information only at the grid itself (i.e., no communication is needed), the model parallelized very well. In fact, for uniform distribution of Lagrangian parcels, it gave perfect parallelization. 


\section{Program for Climate-Model Diagnosis and Intercomparison (PCMDI)}

\section{PROGRAM FOR CLIMATE-MODEL DIAGNOSIS AND INTERCOMPARISON}

GATES, W. LAWRENCE

\section{LAWRENCE LIVERMORE NATIONAL LABORATORY}

$\begin{array}{ll}\text { FY } 1993 & 3805 \\ \text { FY } 1992 & 3690 \\ \text { FY } 1991 & 3191\end{array}$

$10 / 01 / 92-09 / 30 / 93$

Objective: To develop a method for the systematic determination of significant, future, regional climate changes resulting from increased greenhouse gases and to understand these changes in terms of the physical processes portrayed in climate models.

Product: Climate-model documentations and databanks of both model simulations and observed climatological data; advanced software for data storage, diagnosis, and visualization; effective and flexible data-management systems and protocols; and comprehensive summaries of the performance capabilities and systematic errors of current climate models.

Approach: As part of the International Atmospheric Model Intercomparison Project (AMIP), PCMDI will develop an extensive archive of the results of climate models run under standardized conditions, including the output from 30 state-of-the-art U.S. and foreign atmospheric GCMs. This effort will include active participation in several AMIP diagnostic subprojects focused on specific aspects of model performance. A suite of sensitivity and predictability studies including the effects of resolution and feedback will be made from simulations with selected atmospheric GCMs of the European Cen- tre for Medium-Range Weather Forecasts (ECMWF) and other institutions. To validate these models, PCMDI will develop a comprehensive model-compatible database of observed data from both conventional sources and reanalysis efforts and will develop an extensive array of diagnostic software for use in model analysis, including advanced statistical software for the detection of climate change. In cooperation with the modeling community, PCMDI will perform diagnostic studies with coupled atmosphere-ocean GCMs as part of an emerging experimentation and diagnostic program on interdecadal climate change in response to transient greenhouse-gas concentrations.

Results to Date: Substantial progress has been made in the international AMIP: 25 of the 31 participating modeling groups have now completed the 10-year integration, and for 13 of these the standard monthly averaged output has been assembled. PCMDI has also begun the assembly of the simulations' history in standard (DRS) format and has made major improvements in both graphics/visualization software and database management.

Studies of the effects of model resolution on GCMs of the ECMWF have shown that resolution of at least T42 $\left(\sim 3^{\circ}\right.$ latitude) is required to satisfactorily depict the large-scale features of seasonal climate. The primary effect of higher resolution is to add detail in mountainous and coastal regions, especially in terms of precipitation. With an ensemble of ECMWF AMIP runs, research on climate variability has shown that much of the seasonal and interannual variability of regional precipitation may not be predictable (at least in terms of prescribed sea-surface temperature). Research on the identification of climate change has resulted in a new statistical method for climate-change detection that will also be useful in the validation and intercomparison of models. 


\section{QUANTITATIVE LINKS}

\section{Quantitative Links Program}

The sense of science is that greenhouse gases can theoretically cause warming, but the state of science is one of incomplete model prediction and inconsistent observations. Scientists and politicians alike seek verification that the climate is, indeed, being changed and that the cause is an increase of greenhouse gases. However, convincing evidence is evasive, and the lack of certitude about cause, magnitude, and timing of climate change is scientifically unsatisfying.

Unequivocal detection of climate change, including greenhouse warming, is urgently needed. Unambiguous data and scientifically valid approaches do not now exist. The Quantitative Links Program seeks to resolve this problem.

Research Objectives. The objective of the Quantitative Links research program is to establish the relationships between and processes controlling (1) changes in atmospheric composition (particularly $\mathrm{CO}_{2}$ concentration), (2) changes in radiative fluxes, and/or (3) changes in local temperature and longterm changes in global temperature (or other global changes in climate that may result from changes in atmospheric composition). A broad range of research on the linkages between changes in atmospheric composition and global change is conducted. Measurements (field and laboratory) to quantify the relationships are the primary focus.

Improved ability to project future climate will best be achieved by the acquisition, assembly, and analysis of climate data; by the further development, analysis, and verification of climate models; and by observational studies of climatic processes. This ability is a central goal of the Quantitative Links Program.

\section{Program Manager}

Michael Riches

Environmental Sciences Division

Department of Energy, ER-74

Washington, DC 20585

(301) 903-3264

Internet:

mike.riches@mailgw.er.doe.gov

\section{Atmospheric Radiation Measurement (ARM) Program}

ARM is a key component of the Department's research strategy to address global climate change. The Program is a direct continuation of DOE's decade-long effort to improve GCMs and to provide reliable simulations of regional and longterm climate change in response to increasing greenhouse gases.

The ARM Program is a highly focused observational and analytical research effort that compares observations with model calculations in the interest of accelerating improvements in both observational methods and GCMs. During the ARM Program, DOE is collaborating extensively with existing Global Change programs at other agencies.

The ARM Cloud and Radiation Testbed (CART) facility in the U.S. Southern Great Plains locale began operation as scheduled in the spring of 1992. The second ARM site, in the tropical western Pacific Ocean, is scheduled to begin operation in late 1994 or early 1995. The third ARM site, on the North Slope of Alaska, is scheduled to begin operation in late 1996 or early 1997. Data from each of the sites is being integrated at a central 
data system. Data is readily available to the ARM Science Team in near-real-time and is tailored to each of the Science Team projects. Data for the general science community is made available through a data archive established at ORNL.

Research Objectives. The objectives are to quantitatively describe the radiation balance from the surface to the top of the atmosphere, to determine the atmospheric characteristics responsible for this balance, to improve the parameterization of the formation and evolution of clouds in climate models, and to operate an experimental testbed for testing process models used in GCMs and for providing satellite ground-truth measurements.

Research Questions. The research involves a network of ground-based remote-sensing instruments along with campaign studies that use aircraft and tethered platforms. Measurements include vertical profiles of temperature, water vapor, trace gases, aerosols, and solar and infrared radiation. The ARM data provide the testbed for the process models representing the cloud-climate feedbacks in the currently available GCMs as well as in the future climate-change-prediction models of regional-scale resolution.

\section{Program Manager}

Peter W. Lunn

Environmental Sciences Division

Department of Energy, ER-74

Washington, DC 20585

(301) 903-4819

Internet:

peter.lunn@mailgw.er.doe.gov 


\section{Research Summaries}

\section{Quantitative Links Program}

\author{
MONITORING THE RESPONSE OF THE \\ UPPER TROPOSPHERE/LOWER \\ STRATOSPHERE TO A GREENHOUSE-GAS \\ SCENARIO \\ COX, STEPHEN K. \\ COLORADO STATE UNIVERSITY
}

$\begin{array}{lrr}\text { FY } 1993 & 70 \\ \text { FY } 1992 & 179 \\ \text { FY } 1991 & 150\end{array}$

07/01/93-06/30/94

Objective: To develop a system capable of simultaneously monitoring the increased $\mathrm{CO}_{2}$ concentrations and signals of global warming with radiometric methods.

Product: The observations necessary for advancing climate models.

Approach: Three main components of a climate change scenario will be monitored. The method, which pays particular attention to the role of changing amounts of $\mathrm{CO}_{2}$, is based on field measurements of the downwelling radiation field with a moderate-resolution interferometer. The relative changes in the zenithviewed atmospheric radiance over time and the ratios of the radiance at two observation angles will be examined, and information on the concentration of $\mathrm{CO}_{2}$ and the resulting changes in $\mathrm{CO}_{2}$ emission temperatures will be extracted. A more detailed analysis of the data will provide an estimate of variation in $\mathrm{H}_{2} \mathrm{O}$ emission and cirrus cloud cover as well. By monitoring these three climatic modulators, the ability to establish the linkages of increasing $\mathrm{CO}_{2}$ concentration to climate change will be substantially enhanced.

Results to Date: The path-characterization algorithm for FASCOD was acquired from Phillips Laboratory, U.S. Air Force Geophysics Directorate, to apply moreconventional analysis to deriving the temperature and moisture fields from the interferometric data. The algorithm has been modified to include instrumentation and software effects introduced by the interferometer and calibration procedures and has been implemented on both a RISC workstation and a 486-PC. This algorithm is being tested for application to temperature and gasconcentration retrieval from clear-sky interferometric data collected at a high-altitude mountain site; a midlatitude, low-altitude site; and a subtropical oceanic site. A second interferometer and solar-tracking system have been acquired to extend the observation capability to near-infrared solar radiation transmitted through clear sky and cirrus cloudiness.

\section{DATABASE SUPPORT TO ASSIST IN QUANTIFYING THE LINK BETWEEN CHANGES IN ATMOSPHERIC COMPOSITION AND CLIMATE CHANGE}

CUSHMAN, R. M.

OAK RIDGE NATIONAL LABORATORY

\begin{tabular}{lr} 
FY 1993 & 525 \\
FY 1992 & 525 \\
FY 1991 & 511 \\
\multicolumn{2}{r}{$10 / 01 / 92-09 / 30 / 93$}
\end{tabular}

Objective: To provide quality-assured data sets and other information needed for quantifying the links among atmospheric chemistry, the Earth's radiative balance, and climate.

Product: Compiled or acquired numeric data packages (NDPs) consisting of written documentation and machine-readable data files available and distributed free of charge through a variety of media, including 9-track magnetic tape, CD-ROM, IBM-formatted floppy diskettes, and an anonymous file transfer protocol (FTP) area over Internet; and a four-volume series of reports resulting from the translation of scientific foreignlanguage literature. 
Approach: (1) A data set suitable for long-term climate analyses will be compiled, documented, and distributed with the involvement of scientists from the National Oceanic and Atmospheric Administration's National Climatic Data Center and Environmental Research Laboratories. The resulting data set will be called the Global Historical Climatology Network (GHCN). (2) Climaterelated data sets from the former Soviet Union will be acquired, inspected, and documented. (3) A priority list, based on a survey of climate researchers, of the most important extant climate-related data sets needed for quantifying the link between radiative balance and atmospheric temperature will be developed. (4) A series of technical reports will be produced based on the translation into English of foreign-language literature important to the Quantitative Links Program.

Results to Date: Version 1.0 of the GHCN was compiled and documented during the previous two FYs and was published in July of 1992. Through the first half of FY 93, the Carbon Dioxide Information Analysis Center (CDIAC) had responded to 131 requests for the GHCN data set. During FY 93, new algorithms were developed that eliminate duplicate station records, identify inhomogeneous time series, and pinpoint dataprocessing errors. Algorithms were developed to adjust inhomogeneous time series and additional station records were acquired for inclusion in Version 2.0 of the GHCN. Algorithms were constructed to compare neighboring stations to one another to isolate moresubtle data-processing problems.

The quality assurance and review were completed for a climate data set containing long-term, monthly-meantemperature and total-precipitation data for 223 stations in the former USSR. These data have been fully documented and are now available as NDP040 from CDIAC.

Data sets identified in the survey of climate researchers were pursued. One of those data sets, aerosol optical depth measurements from Rattlesnake Mountain Observatory, has been sent to CDIAC and is currently going through the normal data-review and documentation process. This NDP is expected to be completed in early FY 94. Other data sets identified from the survey are being pursued, including historical cloud data, in situ and lidar measurements of stratospheric aerosols (e.g., optical depth and solar transmittance), and surface sensible and latent heat fluxes.

The second of the four-volume series of Russian scientific literature translations is near completion. This volume will offer selected translations of cloud-related publications pertinent to global climate change. The first volume, published in September of 1992, provided translations of abstracts dealing with surface energy budgets. Through the first half of 1993, CDIAC had responded to 20 requests for this first volume.

\section{OBSERVATIONAL ANALYSIS OF CLIMATE FEEDBACKS AND TRENDS}

$$
\text { DEL GENIO, ANTHONY D. }
$$

\section{GODDARD INSTITUTE FOR SPACE STUDIES}

$\begin{array}{lr}\text { FY } 1993 & 150 \\ \text { FY } 1992 & 0 \\ \text { FY } 1991 & 0\end{array}$

$$
07 / 15 / 93-07 / 14 / 94
$$

Objective: To reduce the current uncertainty in global climate sensitivity through analysis of observations and GCM simulations of currently unconstrained feedback processes, and to define useful climate parameters and regions for detecting climate changes in the 1990s.

Product: Constraints for climate models that will reduce uncertainty in linking increasing trace gases to climate change.

Approach: Global observations of several parameters that are central to the detection and understanding of long-term climate change from an increasing greenhouse effect will be analyzed. This study will focus primarily on feedbacks caused by upper-troposphere water vapor, cloud cover, and cloud optical thickness. The ISCCP and SAGE II data sets will be the primary sources of information on these variables, supplemented by available data from Tropical Rainfall Measuring Mission (TRMM) ground truth, the special sensor microwave/ imager (SSM/I), and ARM. First, these data sets will be analyzed for evidence of correlated variations on seasonal or interannual time scales that reveal the sense of climate feedbacks and the physical mechanisms that determine them. GCM cloud and convection parameterizations will then be tested for their ability to reproduce these feedbacks, and their sensitivity to fixed SST perturbations will be evaluated. The final data sets, will approach decadal length, making it feasible to search for any characteristic regional patterns of long-term change. 
Results to Date: ERBE shortwave cloud-forcing anomalies for the $1987 \mathrm{El} \mathrm{Niño/Southern} \mathrm{Oscillation}$ (ENSO) event in the west Pacific have been shown to be large and uncorrelated with the small sea-surfacetemperature anomalies in that region. A correlation exists in the central/east Pacific, but in both regions, time series of sea-surface-temperature tendency are uncorrelated with time series of shortwave cloud forcing. The tendency, however, is strongly correlated with estimated latent heat fluxes over large parts of the tropical Pacific.

Comparison of two high-cloud data sets indicated that when the differing observation path lengths between a limb-extinction instrument (SAGE II) and nadir-viewing retrievals (ISCCP) are taken into account, the two data sets compare extremely well. We have shown that SAGE II measures a physical top to the cloud that is above the radiative top inferred by ISCCP.

A preliminary version of the GISS GCM with a prognostic cloud-water-budget parameterization and interactive optical properties has been shown to have lower climate sensitivity to fixed sea-surfacetemperature perturbations than did the previous version with prescribed optical thicknesses; the cloud feedback in the new model is slightly positive. Both high and low clouds contribute to the feedback difference.

\section{PARALLEL ATMOSPHERIC CLIMATE MODEL}

DRAKE, JOHN B.

\section{OAK RIDGE NATIONAL LABORATORY}

$\begin{array}{ll}\text { FY 1993 } & 800 \\ \text { FY 1992 } & 835 \\ \text { FY 1991 } & 778\end{array}$

$$
10 / 01 / 92-09 / 30 / 93
$$

Objective: To provide a usable parallel implementation of the Community Climate Model, CCM2, for use by modelers, to produce algorithmic improvements suitable for the construction of advanced climate models that couple atmospheric and other components of a climatesimulation system, to produce algorithm libraries and prototype software to aid in the development of parallel climate models, and to sponsor workshops for sharing information among the climate-modeling community.
Product: The advances in numerical methods and parallel processing techniques required to support the modeling of the atmospheric component of the climate system on advanced computing architectures (i.e., massively parallel computers) and implementations of advanced climate models on parallel computers.

Approach: An atmospheric GCM-based climate model that uses the spectral-transform method will be improved and developed. The NCAR Community Climate Model will be used as a base for parallel implementations and to define the fundamental standard for improving parallel climate simulations.

New numerical methods for dynamics and novel parallel algorithms for climate-process computations will be tested as candidates for inclusion in the climate model with the nonlinear shallow-water equations on the sphere. The numerical accuracy and efficiency for dynamics calculations and the parallel performance and suitability for massively parallel computation will be investigated for a variety of numerical methods.

Portable parallel implementations of the CCM2 and follow-on models will be made available for use by the CHAMMP research community on High-Performance Computing Research Center (HPCRC) parallel computers in coordination with the CHAMMP program office and the DOE Office of Scientific Computing (OSC). Portability between massively parallel computers will be based on message-passing implementations and the use of tools like PICL and PVM. Software tools will be modified or developed, as the need arises, to support the research efforts or to provide production environment facilities.

Periodic workshops will be organized, and software modules of interest will be made available.

Results to Date: A subhemispheric Eulerian transport and transformation model for tropospheric sulfate aerosol, driven by operational meteorological data, has been developed and tested. The spatial domain is the North Atlantic and surrounding continents. The grid-cell dimension is $1.125^{\circ}$ with 15 levels between the surface and 100 mbar. Modeled sulfate concentrations exhibit high variability on a spatial scale of hundreds of kilometers and a temporal scale of days. Monthly average calculations will inevitably miss much spatial as well as temporal detail. Calculated daily average sulfate concentrations closely reproduce observed concentrations at locations spread widely over the model domain. 
The model indicates that North American sulfate can be transported over substantial reaches of the North Atlantic. Model outputs of the column burden of sulfate are suitable for comparison with satellite observations of clear-sky and cloud albedo as a function of time and location.

The consequences of the greatly differing residence times of anthropogenic $\mathrm{CO}_{2}$ (mean residence time $\sim 100$ years) and of anthropogenic sulfate aerosol (mean residence time $\sim 1$ week) on present and expected future climate forcing by these substances have been assessed. The present situation in which the forcing by sulfate is comparable to that by $\mathrm{CO}_{2}$ is a consequence of the steeply increasing rates of emissions during the industrial era. The analysis shows that the offset of greenhouse warming caused by light scattering by sulfate aerosols is transient and that the long-term influence is inevitably one of warming.

\section{ICRCCM PHASE II: VERIFICATION AND CALIBRATION OF RADIATION CODES IN CLIMATE MODELS}

ELLINGSON, ROBERT G.

\section{UNIVERSITY OF MARYLAND}

$\begin{array}{lr}\text { FY 1993 } & 274 \\ \text { FY 1992 } & 0 \\ \text { FY 1991 } & 0\end{array}$

$07 / 01 / 93-06 / 30 / 94$

Objective: To establish an absolute standard against which to compare models.

Product: More-accurate radiation models for use in parameterizing climate models.

Approach: A comprehensive program of measurement and analysis called SPECTRE (Spectral Radiance Experiment) will be carried out. SPECTRE will establish an absolute standard against which to compare models to remove the hidden variables (unknown humidities, aerosols, etc.) that radiation modelers have invoked to excuse disagreements with observation. This removal will lead to more-accurate radiation models for use in parameterizing climate models, which in turn play a key role in the prediction of $\mathrm{CO}_{2}$ /trace-gas greenhouse effects.
The major part of SPECTRE will be two surface spectrometer teams and the RASS (radio acoustic sounding system) for temperature profiling.

Many other instruments, such as lidars, will be available because SPECTRE is designed as a subprogram of the FIRE Cirrus II project. And by coordinating with FIRE, SPECTRE will have a unique opportunity to contribute not only to the understanding and modeling of trace-gas greenhouse effects but also the cirrus greenhouse effect.

The ability of the radiation codes used in climate models to simulate the real atmosphere will be evaluated by conducting another round of model intercomparisons. This time the model results will be compared with observations taken during SPECTRE as well as with those of the other models.

Results to Date: Clear-sky spectra of the downwelling radiance at the surface observed during the SPECTRE have been compared with FASCOD3P and with narrowand-broad band radiation models. In general, the lineby-line (LBL) model captures most of the features in the observed clear-sky spectra to within the absolute accuracy of the observation, although there is a tendency for the model to under- and overestimate the observed radiance in some portions of the 8-to-12micron region. Spectrally integrated observations and LBL calculations have also been compared 'with calculations from models used in GCMs. Large differences have been found between some models and the observations, although the differences tend to be largely systematic. Clearly, some models have deficient parameterizations of the water-vapor continuum, and these will have to be changed for the models to yield correct radiances and fluxes over the full range of atmospheric conditions. Our initial findings indicate that FASCOD3P may be used to calibrate the GCM radiation model calculations of the downward flux at the surface to within about $4 \mathrm{~W} / \mathrm{m}^{2}$ for midlatitude winter conditions. 


\section{STATISTICAL EXAMINATION OF CLIMATOLOGICAL DATA RELEVANT TO GLOBAL TEMPERATURE VARIATIONS}

\author{
GRAY, H.; GUNST, R.; and WOODWARD, W. \\ SOUTHERN METHODIST UNIVERSITY

$\begin{array}{lr}\text { FY } 1993 & 200 \\ \text { FY } 1992 & 0 \\ \text { FY } 1991 & 0\end{array}$

$07 / 15 / 93-07 / 14 / 94$

Objective: To construct a state-of-the-art statistical model of global temperatures.

Product: $A$ better identification and understanding of the variables and the change in the variables associated with $\mathrm{CO}_{2}$-induced climate change.

Approach: Models for measures of mean global temperature change will be developed with state-of-the-art statistical techniques, appropriate new methods, and a variety of databases consisting of relevant explanatory variables. Attention will be given to the integrity of existing data as well as to the definition and measurement of global temperature change. The hypothesis of a recent warming trend will be tested to determine whether such a trend, if it exists, is forecast to continue. The impact on warming of changes in the explanatory variables will be investigated, and the contributions of each of the explanatory variables to the cycles, trends, and random variations in the warming/cooling process will be ascertained. Data sources will include not only recent records but also biological and chemical data collected from archeologic and geologic studies.

A statistical model will be constructed to relate global temperature to a collection of exploratory variables. Appropriate multivariate models, including the possibility of long-memory processes, will be developed and used.

Results to Date: While the temperature-anomaly series have a tendency to increase over the past 100 years, we previously showed that similar realizations could be generated from a variety of models. We have been using a bootstrap procedure to determine whether a trend-plusnoise process (which predicts the trend to continue) or an autoregressive process (which predicts the trend will not continue) is more appropriate for the temperature series. We are also examining a variety of time-series models that allow the inclusion of explanatory variables in the modeling of global temperatures.

A long-term goal of this research is to develop spatial statistical models for the fitting of global warming data using kriging procedures. The most challenging aspect of kriging is the estimation of covariance matrices with semivariogram models. Statistical methods based on nonlinear least squares are being developed to provide a general framework for the estimation of semivariogram models. Special adaptations of the methods are being developed to better identify key model parameters, such as the range and the sill. Anisotropic methods are also being developed. Theoretical and empirical properties of the methods are being studied as are comparisons with existing methods currently in use.

\section{THERMOHALINE CIRCULATIONS AND GLOBAL CLIMATE CHANGE}

\author{
HANSON, HOWARD P.
}

\section{UNIVERSITY OF COLORADO}

$\begin{array}{lr}\text { FY } 1993 & 165 \\ \text { FY } 1992 & 0 \\ \text { FY } 1991 & 0\end{array}$

$$
07 / 15 / 93-07 / 14 / 94
$$

Objective: To investigate the sensitivity of the thermohaline circulation of the North Atlantic to changes in surface forcing that may be expected with $\mathrm{CO}_{2}$-induced global warming.

Product: An improved quantitative understanding of the long-term changes in global temperature that may be expected to occur with increasing concentrations of $\mathrm{CO}_{2}$ in the atmosphere and improved estimates of the ocean's ability to dissolve $\mathrm{CO}_{2}$.

Approach: A potentially important feedback between the rate of $\mathrm{CO}_{2}$ uptake by the ocean and the production of cold water in the North Atlantic will be examined, and the role of this feedback in the rate of global warming will be assessed.

Simple sensitivity studies will address the role of hydrological forcing vs thermal forcing in the model. The role of mesoscale eddies in the annual cycle of 
mixed-layer deepening and retreat will be investigated. Salinity, topography, and realistic coastlines will be explicitly included in the calculations. The feedback process will then be addressed, and the climate sensitivity to the feedback will be assessed from the model.

The problem of ocean-climate interactions will be tackled numerically.

Results to Date: Numerical experiments with the hybrid isopycnic/mixed-layer model of the North Atlantic continued to explore the sensitivity of thermocline ventilation associated with the annual cycle of the mixed layer to variations in atmospheric forcing on a variety of scales. Previous results, which suggested that synoptic-scale variations can have a strong influence on the oceanic annual cycle, were examined in more detail with the goal of testing regional variations in this sensitivity. This work was guided by previous results that determined regions of the North Atlantic in which the oceanic mixing potential (defined by the depth to which atmospheric forcing is able to mix surface water) is large. These regions were found to be especially sensitive to synoptic-scale variations in atmospheric forcing; they include broad areas of the North Atlantic just south of Greenland and over the Gulf Stream. In addition, the timing of the synoptic variations is important in affecting changes in thermocline ventilation, with spring storms (when the mixed layer is retreating) providing the strongest influence. A comparison of model results with observations from the Comprehensive Ocean-Atmosphere Data Set (COADS) suggests that including synoptic variability in the atmospheric forcing is necessary to reproduce the observed behavior of the North Atlantic.

\section{CLOUD AND OCEAN EFFECTS ON GREENHOUSE WARMING}

HOFFERT, MARTIN 1.

\section{NEW YORK UNIVERSITY}

$\begin{array}{ll}\text { FY } 1993 & 218 \\ \text { FY } 1992 & 228 \\ \text { FY } 1991 & 217\end{array}$

$$
\text { 07/15/93-07/14/94 }
$$

Objective: To investigate effects of global cloudiness variations on global climate and their implications for cloud feedback and to develop and apply NYU transient climate/ocean models, with emphasis on coupled effects of greenhouse warming and feedbacks by both the clouds and oceans.

Product: A quantitative assessment of how transient climate change may be hidden by cloud feedbacks and ocean thermal delay.

Approach: Cloud, surface-temperature, and ocean data sets, as interpreted by a focused climate/ocean model, will be used to develop a cloud-radiative-forcing scenario for the past 100 years and to assess the transient climate response; to narrow key uncertainties in the system; and to identify those aspects of the climate system most likely to be affected by greenhouse warming over short, medium, and long time scales. Apart from short-term transients on the decadal scale, a better understanding will be sought of latent climatic effects (i.e., global climatic and environmental changes to which our planet may be effectively committed, but which are temporarily hidden by thermal storage by the oceans). Effects of cloud radiative forcing on global climate response will be assessed with an extension of the method previously employed to assess solarvariability effects on climate. This simplified model will assist in developing an understanding of the key processes before investigating with the GCMs.

Results to Date: A method has been developed for calibrating global climate sensitivity from paleoclimate data on radiative forcing and temperature response of very different climates: The warm middle Cretaceous (100 million years ago) was some $10^{\circ} \mathrm{C}$ warmer than today, and the Last Glacial Maximum (about 20,000 years ago) was more than $3^{\circ} \mathrm{C}$ colder. We found that a $\mathrm{CO}_{2}$-doubling climate sensitivity of $2.3^{\circ} \mathrm{C}$ and a standard deviation of $\sim 0.5^{\circ} \mathrm{C}$ explain both of these very different climates in the midrange of current GCM results. These results, together with ERBE satellite data over clear-sky regions, imply a slightly positive cloud radiative feedback contributing $<15 \%$ to the global climate sensitivity.

Paleoclimate data from the former USSR on zonal mean temperature distributions of ancient climates is being applied to better understand and predict the changes of poleward heat flow when climate changes. Current GCMs appear to systematically underpredict the increase in the equator-to-pole temperature gradient associated with equilibrium global warming. We are currently investigating whether this is caused by some underlying 
problem with model parameterizations of meridional heat flow by the atmosphere and oceans or by failure to properly account for processes that stabilize tropical temperatures.

\section{LINKAGE OF ANTHROPOGENIC AEROSOL TO CLOUDS AND CLIMATE}

HUDSON, JAMES G.

DESERT RESEARCH INSTITUTE

$\begin{array}{lr}\text { FY } 1993 & 120 \\ \text { FY } 1992 & 0 \\ \text { FY } 1991 & 0\end{array}$

$07 / 15 / 93-07 / 14 / 94$

Objective: To quantitatively establish the definition of cloud condensation nuclei (CCN).

Product: $\mathrm{A}$ basis for separating the relative effects on cloud microphysics of natural and anthropogenetic CCN and a definition of the nucleus supersaturation range appropriate to stratus clouds and of the relative efficiency of nuclei to activate cloud formation.

Approach: Cloud condensation nuclei will be defined to distinguish between the role of natural and manmade nuclei in cloud formation. The result will determine the linkage between global air pollution and cloud microphysics, which will enable realistic estimates to be made of this possible anthropogenic effect on cloud radiation balance and climate.

A measurement program for $\mathrm{CCN}$ based on proven techniques will be carried out. Cloud formation, radiative properties, and precipitation characteristics depend on the number, size, and chemical properties of the CCN. Data will be collected on the size and number. The $\mathrm{CCN}$ will be measured with an instantaneous $\mathrm{CCN}$ spectrometer, which will provide a detailed $\mathrm{CCN}$ spectrum over the entire range of interest to cloud physics. The nuclei actually active in cloud formation will be subtracted out from the total aerosol.

Through identification of the $\mathrm{CCN}$, the cloud-activation efficiency will be determined over a given size range and other radiative properties of the CCN.

Surface and aircraft measurements will be used to collect CCN/aerosol samples for analysis. Wet and dry aerosols will be separated electrostatically, and chemical as well as water-solubility properties of the materials will be determined.

Results to Date: Cloud-droplet data from ASTEX (the Atlantic Stratocumulus Transition Experiment held in June of 1992) showed that polluted clouds not only have higher concentrations and smaller droplets but that the droplet-size spectrum is also substantially narrower. This result is not predicted by theory, but we can now offer an explanation. The slope of the CCN spectrum is also lower for polluted air $(\sim 0.4)$ than for clean air $(\sim 1.3)$, and this distribution interacts with variations in updraft velocity to produce less variability in droplet concentrations and size distributions in polluted clouds. The narrower droplet spectra leads to an inhibition of the coalescence process, and this stabilizes the clouds. The subsequent increase in cloud lifetimes could be as much of a climatic effect as the effect of $\mathrm{CCN}$ on cloud radiative properties.

During July 1993, measurements were made in the SOCEX (Southern Ocean Cloud Experiment) project. CCN concentrations in the Southern Hemisphere are indeed very low $\left(<50 \mathrm{~cm}^{-3}\right)$, as expected. Particle volatility measurements indicated that the $\mathrm{CCN}$ in the Southern Hemisphere do not have a nonvolatile core as do most of the CCN in the Northern Hemisphere; this finding may indicate significantly different sources for the CCN. Size-vs-supersaturation measurements seemed to be similar in both hemispheres indicating little difference in the sizes of $\mathrm{CCN}$.

\section{NETWORK-BASED SOLAR AND \\ METEOROLOGICAL CHARACTERIZATION \\ OF CLOUD-RADIATION EFFECTS IN GLOBAL CLIMATE MODELING}

\author{
LAULAINEN, N., and MICHALSKY, J. \\ PACIFIC NORTHWEST LABORATORY and \\ STATE UNIVERSITY OF NEW YORK AT \\ ALBANY
}

$\begin{array}{lr}\text { FY } 1993 & 250 \\ \text { FY } 1992 & 275 \\ \text { FY } 1991 & 254 \\ & \\ & 10 / 01 / 91-09 / 30 / 92\end{array}$

Objective: To use ground-based measurements in concert with satellite results to characterize cloud 
radiative forcing for a variety of cloud types and structures over a limited geographical area, to track changes in cirrus frequency and optical depth, and to track changes in aerosol optical depth and its wavelength dependence.

Product: A better understanding of how the atmosphere, especially clouds, forces the top-of-the-atmosphere infrared and solar radiation to their observed values; whether cirrus clouds are increasing in the nonovercast sky condition; and whether the tropospheric aerosol burden is changing with time and how it affects cloudy-sky albedo.

Approach: Clouds mask about half the Earth's surface at any given time. Therefore, it is important to know how clouds interact with outgoing longwave (infrared) and incoming shortwave (solar) radiation to regulate the heating of the planet. To better understand how these climatically opposed effects vary in time, with cloud type and structure, and to a limited extent with geography, a nine-station regional network will be established in the eastern United States. Each station will be equipped with standard instruments for measuring ambient temperature, total precipitation, and relative humidity. Observations of diffuse horizontal, total horizontal, and direct normal solar irradiance are the primary solar measurements and will be made with a multifilter/detector rotating shadowband radiometer (MFRSR) in six narrow-wavelength bands and in one unfiltered broadband that corresponds to the standard shortwave instruments. For increased stability and reliability, all three solar radiation components will be determined with the same sensor for each wavelength band. To aid in the interpretation of these quantities, supplemental measurements of downwelling infrared radiation on a horizontal surface and local surface albedo (upwelling solar radiation) will also be made. All observations at each site will be recorded on a 16-channel data logger. Several climate-sensitive parameters will be derived from the solar observations, including cloudiness, cloud optical depth, cloud type (to some extent), and aerosol optical depth and wavelength dependence.

Results to Date: The nine-station Quantitative Links Network (QLN) has been in operation for 2 years, recovering about $90 \%$ of the data. Most of the data loss was associated with scheduled instrument calibrations and retrofits for improved operation of the MFRSR. Units are interrogated daily to check status and to retrieve data. Data-reduction and -analysis methods have been developed, including routines to (1) perform cosine-response corrections on the direct irradiance data, (2) perform automated Langley regressions to calculate aerosol optical depths and calibration constants for the six filtered detectors, and (3) apply general calibration algorithms to produce engineering data. Monitoring the decaying Mt. Pinatubo stratospheric aerosol cloud continued. Retrievals of column water vapor with the 940-nm wavelength channel were also begun. Because water vapor is the most significant greenhouse gas, the reliability of the retrievals are being compared with balloon sondes and microwave radiometers measurements at the ARM Southern Great Plains CART site. UV-B radiation measurements were added at three QLN sites in a cooperative program with the USDA.

\section{SIGNAL AND NOISE IN GLOBAL-WARMING DETECTION}

NORTH, GERALD R.

TEXAS A\&M UNIVERSITY

$\begin{array}{lr}\text { FY } 1993 & 110 \\ \text { FY } 1992 & 0 \\ \text { FY } 1991 & 0\end{array}$

$07 / 15 / 93-07 / 14 / 94$

Objective: To estimate the length of climatologic record required to identify a global-warming signal over natural variability.

Product: An understanding of the adequacy of the historical instrument record to resolve the issue of signal and noise in the matter of global warming.

Approach: The global-trend-observing system will be studied by a statistical analysis of the variability about trends into their natural and random sampling components. Idealized mathematical models, such as noise-forced energy-balance models and GCM runs of idealized planets, will be used to produce long-timeseries data for analysis. These data will provide the long record required to assess how long a data set may be required to determine natural variability. Although this is not real data, the approach seeks understanding before more-complicated climate models are applied to the task.

A formalism will be used for sampling error analysis. The technique can be applied to physical fields 
exhibiting random fluctuations over a space-time spectrum. For this research, the technique will be applied to the space-time spectrum of the surfacetemperature field. The outputs from a simple energybalance model and from a simplified version of the NCAR GCM will be analyzed.

Results to Date: We have addressed the sampling-error problem for estimation of the Earth's global average temperature with a finite number of point gages. This analysis has been accomplished for a sequence of ideal cases approaching the most realistic case with real land-sea geography. A formula was developed for the mean-square error in terms of the second moments of the temperature field. The results were presented compactly in a spectral form. For an ideal planet where the statistics are rotationally invariant, we have been able to solve the problem with $a$ spherical harmonic basis. When the gages are optimally located, the error variance can be as small as $15 \%$ of the natural variance of global averages for about 60 gages. If the gages are not optimally located, the errors can be minimized by optimal weighting. The same class of problems was solved for the planet with realistic land-sea geography. In this case, the empirical orthogonal functions must be used for the basis set. By using EOFs from both data or model simulations, we find again that the errors caused by optimally weighted station data are quite acceptable for about 60 stations.

\section{MODIFICATION OF CLOUD OPTICAL PROPERTIES BY AEROSOLS}

NOVAKOV, TIHOMIR

LAWRENCE BERKELEY LABORATORY

$\begin{array}{ll}\text { FY } 1993 & 342 \\ \text { FY 1992 } & 342 \\ \text { FY 1991 } & 316\end{array}$

$10 / 01 / 92-09 / 30 / 93$

Objective: To experimentally quantitate the links between the optical properties of clouds and atmospheric aerosols, such as sulfate and carbonaceous particles.

Product: A laboratory and field data set to estimate the optical properties of aerosols and clouds.
Approach: The radiative properties of natural clouds will be measured to determine the validity of theoretical predictions of increasing cloud albedo with increasing CCN (particularly sulfate and mineral dust) concentrations. The relative impacts of energy-related, crustal, and biogenic aerosols on the radiative properties of clouds will be determined. The soot content of natural cloud drops will be determined so that the possible role of soot particles in decreasing cloud albedo can be assessed. Results obtained from these field studies will be used to design laboratory simulation experiments with the LBL cloud chamber to identify the physics and chemistry of dominant importance in modifying the optical properties of clouds, with special emphasis on enhanced absorption soot-associated clouds. And existing ambient and source emissions data will be compiled, evaluated, and analyzed to assess the horizontal, vertical, and seasonal variations in global aerosol concentration.

Results to Date: Nonseasalt (nss) aerosol sulfate and organic carbon mass concentrations, mass size distributions, $\mathrm{CCN}$ and $\mathrm{CN}$ number concentrations, and clouddroplet concentrations were measured in anthropogenically influenced marine air masses on El Yunque peak $\left(18^{\circ} 19^{\prime} \mathrm{N}, 65^{\circ} 45^{\prime} \mathrm{W}\right.$; elevation $\sim 1000 \mathrm{~m}$ ) in Puerto Rico. The purpose of these measurements is to establish the relationships between chemical composition of the aerosol and $\mathrm{CCN}$ number concentration and between nss sulfate mass concentration and cloud droplet number concentration. The measured mass size distributions of organic and sulfate aerosol were used to estimate the relative contributions of these species to the $\mathrm{CCN}$ and $\mathrm{CN}$ number concentrations (at $0.5 \%$ supersaturation). $\mathrm{A}$ comparison of estimated and measured $\mathrm{CCN}$ and $\mathrm{CN}$ indicates that organic aerosols may account for a substantial fraction of $\mathrm{CCN}$ and for most of the $\mathrm{CN}$ number concentrations at this site. Droplet concentrations in the cumulus clouds do not show a discernible trend with nss sulfate mass concentrations. In stratocumulus clouds, a small increase $j_{i}$ droplet concentration with nss sulfate mass concentiation was observed. 


\section{THE ROLE OF AEROSOLS AND CLOUD MICROPHYSICS IN CLIMATE AND CLIMATE CHANGE}

PENNER, JOYCE E.

\section{LAWRENCE LIVERMORE NATIONAL LABORATORY}

$\begin{array}{ll}\text { FY } 1993 & 318 \\ \text { FY } 1992 & 318 \\ \text { FY } 1991 & 292\end{array}$

$10 / 01 / 92-09 / 30 / 93$

Objective: To use data analysis and a global model to quantify the effects of aerosols and clouds on the radiative budget of the Earth and climate.

Product: Quantification of the links among aerosols, cloud microphysical properties, and climate.

Approach: Available data will be compared with the predictions of a 3-D global model for aerosols and clouds. Data on individual sulfur species, aerosol concentrations, black-carbon concentrations, and cloud microphysical properties will be used. The global model will primarily be compared with long time series of data for single stations or comparisons to satellite data, but comparison of the model with more short-term episodic data will also provide valuable insight and allow even short-term data to be folded into the analysis. In this way, the data from a single station or even an event can be extrapolated to determine the global impact.

Results to Date: A version of our sulfur aerosol model was completed, and the results from this model were compared with data. The predictions from this model lead to a climate forcing that is higher than two recent 3-D model estimates in part because we include a seasonal variation in sulfate production that reproduces the observations of sulfate in precipitation. The model was used to estimate an upper limit for the indirect effect of sulfate aerosols on the reflection of solar radiation by clouds, assuming an externally mixed aerosol.

We also developed a technique for estimating changes to cloud albedo assuming an internally mixed aerosol. This technique will allow us to estimate a lower limit to the indirect forcing by anthropogenic sulfur aerosols.
OCEAN CLOUD/CLIMATE LINKAGE STUDY

PORCH, WILLIAM M.

LOS ALAMOS NATTONAL LABORATORY
\begin{tabular}{cc} 
FY 1993 & 250 \\
FY 1992 & 250 \\
FY 1991 & 297 \\
\multicolumn{2}{l}{$10 / 01 / 91-09 / 30 / 92$}
\end{tabular}

Objective: To improve the understanding of how marine stratocumulus clouds respond to changes in atmospheric composition and atmospheric warming and thus feed back on climate.

Product: Improved ocean-cloud parameterizations in climate models and ocean data stratification for satellite cloud-trend studies.

Approach: The effect of heating and aerosol nucleation on marine stratocumulus clouds will be determined by studying the effect of ships on cloud development and albedo. Experiments will be conducted under meteorological conditions favorable to ship-trail development in ocean regions where clouds are particularly sensitive to anthropogenic influence. Ships with the potential for ship-trail formation will be followed by research vessels equipped to probe and sample the trails. The results will be used to test and improve dynamic and aerosol-microphysics-based numerical cloud models. The results from the experiments and model studies will be used to provide a matrix of sea-surface-temperature and marineboundary-layer conditions occurring during high-resolution-image-satellite overpasses of these cloud-sensitive regions. This work will aid in the interpretation' and development of trend analysis of cloud fraction, albedo, and atmospheric composition.

Results to Date: The data from the ocean experiment conducted by this project in July 1991 to measure the response of marine stratocumulus clouds to external forcing was analyzed. The forcing mechanisms studied included (1) the effect of large ships in producing ship-trail clouds, (2) the effect of a total solar eclipse on marine stratus clouds, and (3) the effect of a tropical storm on the marine boundary layer at midlatitudes.

Specifically, the satellite-image analysis of our encounters with four ship trails and the effect of the solar eclipse were studied. Improved image analysis 
showed that during the major ship-trail encounter our small research ship had an observable effect on the background cloud field. The effect of the solar eclipse on the marine boundary layer was much more subtle than that observed over land surfaces. The largest effect was a decrease in wind speeds at totality and a rapid change in wind direction. A decrease in air temperatures of 0.2 to $0.4^{\circ} \mathrm{C}$ was observed at $210 \mathrm{~m}$. However, a warming was observed near the bottom of the cloud. This warming could be partly caused by subsidence or latent-heat release. Analysis of ceilometer and Doppler radar measurement indicated that subsidence dominates.

\section{MECHANISMS CONTROLLING THE PRODUCTION AND TRANSPORT OF METHANE, CARBON DIOXIDE, AND DISSOLVED SOLUTES WITHIN A LARGE BOREAL PEAT BASIN}

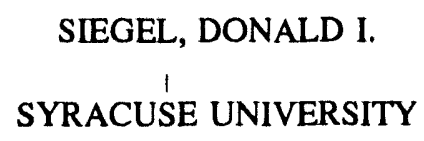

$\begin{array}{ll}\text { FY } 1993 & 200 \\ \text { FY } 1992 & 159 \\ \text { FY } 1991 & 154\end{array}$

$07 / 15 / 93-07 / 14 / 94$

Objective: To determine the degree to which solute and gas movement in deep organic soils is controlled by local ground-water flow systems and their associated vegetation community types and by regional subsurface hydrogeologic settings.

Product: A definition of the potential release of $\mathrm{CO}_{2}$ and methane from boreal peat basins.

Approach: An integrated modeling and field study of solute transport and ground-water flow conditions in the Glacial Lake Agassiz Peatlands of northern Minnesota will be used to investigate the rates of carbon accumulation and loss, as well as fluxes of methane and $\mathrm{CO}_{2}$; the physical and botanical factors controlling the production of methane and $\mathrm{CO}_{2}$ within peat profiles; and the role of hydrogeological processes in controlling the fluxes of gases solutes through the peat.

Hydraulic-head gradients and solute/gas concentrations will be measured in the major vegetation communities of the peatland. The fluxes of dissolved solutes and gases from the peat in each vegetation setting will be determined from these data by mathematical modeling of the advection-dispersion equation, tested by isotopic mass balances on carbon. The results from these detailed site-specific studies will be integrated into the larger peatland setting by relating the variations in solute fluxes to the proportions of each major vegetation type in the larger peatland.

Gases and solutes will be microsampled throughout the depth profile of the peat with standard methods.

Isotopic analysis $\left(\Delta^{13} \mathrm{C}\right)$ will be used to provide evidence for the nrocesses by which methane is produced and transformed within the peat.

Results to Date: Concentrations of dissolved $\mathrm{CH}_{4}$ in peat pore-water decreased between August 1990 and July 1991. The average diffusive flux rate of $\mathrm{CH}_{4}$ decreased from 95 and $89 \mathrm{mg}^{-\mathrm{m}^{-2}}$-day ${ }^{-1}$ in bogs and fens (respectively) in 1990 to 45 and $37 \mathrm{mg}-\mathrm{m}^{-2}$-day ${ }^{-1}$ in 1991. The rate of dissolution of $\mathrm{CH}_{4}$ to the peat porewater decreased by a factor of 10 between the two years ( $\approx 3 \times 10^{-8}$ in 1990 to $0.3 \times 10^{-8} \mathrm{mgL}^{-1} \mathrm{~s}^{-1}$ in 1991). These differences may be an effect of the drought that coincided with the first year of study, or they are possibly seasonal variations in $\mathrm{Ch}_{4}$ profiles, dissolution, and diffusive flux that were observed during two phases of an annual cycle.

Dispersivity studies of peat cores showed that hydraulic conductivity of peat is sensitive to solutes in the pore water. Tracer studies with chloride showed that the hydraulic conductivity of peat increases as the concentration of chloride increases. Consequently, changes in the hydraulic gradient would be expected, as predicted by Darcy's Law.

Profiles of pore water chemistry of major metals changed during the study, lagging behind changes in the hydrology. These time lags result from the time required for the peat to be flushed with pore water.

Darcy calculations with field measurements of hydraulic conductivity suggest it would take about 10 years for a 3-m-thick peat column to be completely flushed. Thus, long-term changes in hydrology will change peat pore water chemistry and possibly affect vegetation communities and peatland evolution; however, shortterm changes in the hydrology should not change the pore water chemistry significantly. 


\section{THE EFFECT OF CLOUD MICROPHYSICS ON CLIMATE SENSITIVITY}

\author{
STAMNES, KNUT
}

\section{UNIVERSITY OF ALASKA}

$\begin{array}{ll}\text { FY } 1993 & 80 \\ \text { FY } 1992 & 80 \\ \text { FY } 1991 & 77\end{array}$

$07 / 15 / 93-07 / 14 / 94$

Objective: To develop cloud radiative parameterizations, based on liquid-water content and cloud-drop-size distribution, for a radiative-convective model.

Product: A more realistic treatment of clouds in climate models by developing methodologies based on cloud microphysics.

Approach: Cloud feedbacks comprise a critical problem to quantitative modeling of the atmospheric response to enhanced trace gases, the current understanding of which is very poor.

This research is focused on the effects of potential changes in cloud microphysics due to a greenhouse warming on the radiative energy budget of the atmosphere. The cloud liquid-water content and dropsize distribution will be used to define cloud radiative properties. Reliable and efficient parameterization of scattering and absorption/emission by cloud droplets will be developed, providing a better understanding of the role of cloud microphysics in the determination of cloud radiative properties. A radiation model including these cloud parameterizations will be used for investigating seasonal and latitudinal effects of the influence of cloud microphysics and liquid-water content.

A detailed, 1-D radiative-convective model will be used to study the role of cloud microphysics in the determination of cloud radiative properties. This radiative-convective model will be used to obtain first estimates of the surface-temperature response to potential changes in cloud microphysics associated with greenhouse warming. It will also be used to assess how cloud droplet size influences cloud/radiation feedback and thereby climate sensitivity. The climatic implications of changes in cloud microphysics in 1-D and multidimensional climate models will be assessed.
Results to Date: A 1-D radiative-convective model with a comprehensive radiative-transfer scheme and an accurate parameterization of cloud optical properties has been developed and used to study cloud microphysics, radiation, and climate interaction. With an improved consideration of convective processes and lower boundary energy balance of latent heat, sensible heat, and radiation, this model is capable of testing the significance of changing cloud-drop-size distributions on the modeling of climate change. The results show that the Earth's ground surface temperature is very sensitive to the changes in the equivalent radius of the clouddrop-size distribution. A sensitivity study with different kinds of clouds shows that for a fixed liquid-water amount, a $10 \%$ decrease in cloud equivalent radius will decrease the equilibrium surface temperature by $3^{\circ} \mathrm{C}$, which is about the same magnitude as the warming expected from doubling of the $\mathrm{CO}_{2}$ concentration. A $15 \%$ increase in cloud water amount or a $1-\mathrm{km}$ decrease in cloud height will yield similar results. Because the model can estimate the latent-heat exchange at the lower boundary, it can be used to study the sensitivity of globally averaged evaporation (precipitation) as well. This sensitivity test is in progress.

\section{DETERMINING THE CHEMICAL COMPOSITION OF CLOUD CONDENSATION NUCLEI}

WILLIAMS, ALLEN L.

ILLINOIS STATE WATER SURVEY

$\begin{array}{ll}\text { FY } 1993 & 420 \\ \text { FY } 1992 & 425 \\ \text { FY } 1991 & 462\end{array}$

07/15/93-07/14/94

Objective: To develop the instrumentation and methods to determine the $\mathrm{CCN}$ chemistry in ambient-air samples.

Product: Data for characterization of CCNs and cloud microphysics in GCMs.

Approach: The CCN spectrum determines to a large extent the cloud-droplet spectra and influences the resulting cloud radiation properties. The view advocated here is that an understanding of the $\mathrm{CCN}$ chemistry is critical to realistic analysis of the role of clouds in climate warming scenarios. 
The appropriate instrumentation and methods to determine the $\mathrm{CCN}$ chemistry in ambient air samples will be developed. Air will be continuously drawn into a flow-type thermal-diffusion cloud chamber to form droplets on the $\mathrm{CCN}$ at precisely controlled supersaturations. The droplets will be immediately removed from the flow stream to minimize contamination and will be collected on a filter for analysis. It is estimated that microgram amounts of CCN material can be collected. Chemical analysis will employ liquid ion chromatography and atomic adsorption. Other techniques will also be investigated.

Results to Date: CCN measurements at a Midwest site reveal the presence of a significant organic contribution to the $\mathrm{CCN}$ compusition. The apparatus partitions the CCN samples into two dry particle size ranges: a largeCCN sample corresponding to particles between $0.1 \mu \mathrm{m}$ and $0.5 \mu \mathrm{m}$ and a small CCN sample corresponding to particles smaller than $0.1 \mu \mathrm{m}$. Currently, the data for the small $\mathrm{CCN}$ is more complete and reveals the composition to be $22 \%\left(\mathrm{NH}_{4}\right)_{2} \mathrm{SO}_{4}, 10 \%$ elemental carbon, and $64 \%$ organic carbon. Crustal elements and $\mathrm{NaCl}$ are conspicuously absent from the samples. The large-CCN samples appear to have a sornewhat larger amount of $\left(\mathrm{NH}_{4}\right)_{2} \mathrm{SO}_{4}$ and less organic material.

Quartz filters, upon which most of the samples have been collected, show some loading on a backup filter placed in series with the primary filter. The backupfilter loading, in contrast to the primary filter, appears to saturate after a few hours. Gas chromatography/mass spectrometry (GC/MS) analysis of methanol extracts reveal phthalates present at comparable levels on primary and backup filters, representing a significant portion of the backup-filter loading and a small portion of the primary-filter organic loading. The phthalates are presumed to be a sampling artifact, which should be eliminated by refinements in the sampling procedure designed to remove the $\mathrm{CCN}$ sample from ambient air stream. The remaining organic material on the primary filters appears to be much less volatile in nature. Attempts at derivitization of the primary-filter loadings to enhance the volatility for GC/MS analysis have revealed the presence of dicarboxylic acids, but the analysis is incomplete.

\section{TECHNICAL REVIEW OF CARBON DIOXIDE RESEARCH INITIATIVE PROPOSALS}

\author{
WOHLPART, A.
}

\section{OAK RIDGE INSTITUTE FOR SCIENCE AND EDUCATION}

\begin{tabular}{lr} 
FY 1993 & 300 \\
FY 1992 & 250 \\
FY 1991 & 349 \\
\multicolumn{2}{c}{$10 / 01 / 91-09 / 30 / 92$}
\end{tabular}

Objective: To provide the technical review of proposals submitted in response to the Carbon Dioxide Research Initiatives and to conduct technical meetings in support of program objectives.

Product: Statistical data and reports that will assist DOE in ensuring the quality of the review process and in making funding decisions.

Approach: Program documents will be provided to potential applicants upon request, proposals will be received and administratively screened, a proposal data base and software to manage the program will be developed, review meetings will be coordinated, the comments and recommendations of review panels will be documented, and other assistance will be provided as required. Technical support for scientific meetings, including meeting logistics and proceedings, will be provided. 


\section{Atmospheric Radiation Measurement (ARM) Program}

\section{AN INTEGRATED CLOUD OBSERVATION AND MODELING INVESTIGATION IN SUPPORT OF THE ARM PROGRAM}

\author{
ACKERMAN, THOMAS P.
}

PENNSYLVANIA STATE UNIVERSITY

$\begin{array}{lr}\text { FY } 1993 & 750 \\ \text { FY } 1992 & 0 \\ \text { FY } 1991 & 665\end{array}$

$11 / 01 / 92-10 / 31 / 93$

Objective: To study cloud formation, cloud properties, and radiation transfer in the atmosphere; to develop data-assimilation models; and to use explicit simulations of small-scale cloud processes for the purpose of developing suitable parameterizations for large-scale atmospheric numerical models.

Product: Information on methods of observation and data assimilation that are suitable for testbed sites of the ARM Program and parameterizations of cloud processes for large-scale atmospheric models.

Approach: With the suite of instruments used in the Pennsylvania State University's cloud observing system (COS), data will be collected for several extended observing periods and analyzed for such quantities as vertical velocity, cloud cover, cloud optical properties, and surface radiation budgets. With these observations, the vertical structure of the atmosphere that is equivalent to the structure simulated at an individual grid point of a mesoscale numerical model or a GCM will described. Methods for 4-D data assimilation will be developed to provide data inputs efficiently to atmospheric models.

Methods will be developed and applied to exchange data between fields produced by a mesoscale model with modules that describe the atmospheric boundary layer (the lower $2 \mathrm{~km}$ of the atmosphere) and detailed cloud processes. Interactions of processes on small scales and the mesoscale will be examined carefully and parameterized as needed for large-scale models. As part of this work, radiative-transfer models will be applied to simulate the interactions between microphysical and radiative processes and will be compared with the measurements of cloud and radiation properties. As bet- ter understanding is achieved, improved parameterizations of cloud processes will be incorporated into available mesoscale models and tested.

Results to Date: None reported.

\section{REMOTE SENSING OF SURFACE FLUXES} IMPORTANT TO CLOUD DEVELOPMENT

BARNES, F.; PORCH, W.; and KUNKEL, K.

LOS ALAMOS NATIONAL LABORATORY and UNIVERSITY OF ILLINOIS

$\begin{array}{ll}\text { FY } 1993 & 300 \\ \text { FY } 1992 & 300 \\ \text { FY 1991 } & 300\end{array}$

$10 / 01 / 92-09 / 30 / 93$

Objective: To determine the scaling relationships of upward fluxes of water vapor and heat from the surface and their connection to variations in surface features and parameters important to cloud formation.

Product: Characterization of the convective and mixing processes over a range of scales and surfaces at experimental sites and subsequent delineation of the influence of these processes on the local radiation budget and cloud processes.

Approach: Aa integrated measurement program will be developed to study the patterns of surface fluxes of water vapor and heat for sites exhibiting a range of surface and meteorological conditions. These measurements will be used to evaluate the appropriate measurement scale of the upward fluxes as they relate to cloud processes and radiation transport.

Collaboration with instrument-development programs funded by ARM will allow detailing the 3-D structure of the lower atmosphere. Observed patterns in the atmosphere will be correlated with both surface vegetable and terrain pattern and with inhomogeneities recorded in the cloud layer at an established CART site.

The spatial and temporal variations in heat and water vapor flux rates will be determined and related to variations in the radiative balance above the site. 
Collaborations will be assisted with mapping the timedependent vertical distribution of water vapor from the surface to the cloud layer.

Remote sensing (e.g., lidar-ceilometers) will be used to monitor cloud cover; these data will be related to cloud cover determined by satellite imagery and/or multispectral digital imaging. The topographic and terrain features will be established with digital maps. Standard ecological features will be measured. Surfacealbedo measurements obtained from aircraft-mounted instruments or remotely controlled platforms will be used.

The scaling characteristics of the heat and evaporative fluxes will be examined over a variety of ranges up to the subgrid scale of mesoscale models. These results will apply to single-column GCMs. The flux fields over an experimental site will be correlated with cloud characteristics and dynamics over the site. Based on modeling studies, the feedbacks between the surface and the atmosphere that contribute to cloud formation over a CART site will be evaluated.

Results to Date: None reported.

\section{EVALUATION OF A NEW GCM-CAPABLE STOCHASTIC CLOUD/RADIATION PARAMETERIZATION THAT USES ARM DATA}

BYRNE, R. NELSON

\section{SCIENCE APPLICATIONS INTERNATIONAL CORPORATION}

$\begin{array}{lr}\text { FY } 1993 & 177 \\ \text { FY } 1992 & 174 \\ \text { FY 1991 } & 0\end{array}$

$03 / 01 / 93-02 / 28 / 94$

Objective: To use ARM cloud morphology and cloud/radiative energy balance data to define the parameters for a new cloud parameterization technique based upon an innovative stochastic radiative-transfer technique.

Product: A validated stochastic cloud/radiation-transfer parameterization for use in GCMs.
Approach: Stochastic radiative-transfer techniques will be adapted from nuclear physics for application to radiative transfer in the presence of clouds. Clouds and clear air will be treated as a two-component stochastic system wherein subgrid radiative transfer through partial cloud cover is a natural consequence. Algorithms that are developed will be evaluated with ARM and satellite data in the Scripps single-column semiprognostic model and used to explore the accuracy required to achieve a specified level of accuracy for atmospheric energy deposition by radiative processes. GCM sensitivity studies will be conducted with NCAR's CCM.

Results to Date: Correction functions to be used in GCMs based on hypothesized cloud size and shape parameters have been calculated, and the effect of these corrections on the output of our stand-in for a GCM, the Scripps single-column model of Somerville and lacobellis, has been computed. Numerical solutions have been computed for a large number of problems with input conditions produced by random choices from specified distribution functions, and the average solutions of these problems have been studied as functions of the distribution functions. The prototype of a radiation-scene generator has been developed, and its feasibility demonstrated. The statistics of chord-length distributions of ellipsoidal clouds have been studied. We are supporting graduate students at Scripps and at UCLA.

\section{SCIENCE TEAM PARTICIPATION IN THE ARM PROGRAM}

CESS, ROBERT D.

\section{STATE UNIVERSITY OF NEW YORK AT STONY BROOK}

$\begin{array}{lr}\text { FY } 1993 & 55 \\ \text { FY } 1992 & 0 \\ \text { FY } 1991 & 52\end{array}$

$11 / 01 / 92-10 / 31 / 93$

Objective: To attend ARM Science Team Meetings and act as an interface between the ARM program and several related NASA projects involving satellite measurements and with a NOAA project wherein ground-based and satellite measurements are compared.

Product: An enhanced coordination between the ARM program and related NASA and NOAA projects. 
Approach: ARM Science Team Meetings will be attended, knowledge gained in other related programs will be shared, and ways of interacting with these programs will be suggested.

\section{A RESEARCH PROGRAM ON RADIATIVE- TRANSFER-MODEL DEVELOPMENT IN SUPPORT OF THE ARM PROGRAM}

\author{
CLOUGH, SHEPARD A.
}

\section{ATMOSPHERIC AND ENVIRONMENTAL RESEARCH, INC.}

$\begin{array}{lr}\text { FY } 1993 & 229 \\ \text { FY } 1992 & 0 \\ \text { FY } 1991 & 213\end{array}$

$11 / 01 / 92-10 / 31 / 93$

Objective: To develop a highly accurate radiativetransport model for scattering and nonscattering atmospheres; to use calculations from this model for parameterizations required for GCM radiative-transport codes; to validate this code; and to support the design, analysis, and interpretation of the CART measurement program.

Product: Improved radiative-transport parameterizations for GCMs.

Approach: Support will be provided to the ARM research program to implement FASCOD3 as a research-grade reference radiative-transfer (RT) model for GCM-related calculations. Improved algorithms and interrelated data for FASCOD3 will be developed to improve the accuracy of RT calculations in both the short- and the long-waivelength regions.

An updated water-vapor-continuum model will be developed, and the algorithms updated to keep pace with new measurements and/or modified water vapor for far-wing line shapes.

Technical support will be provided for the maintenance of the GL HITRAN line parameters database, a transition spectral database incorporating new and improved line parameters, and realistic scattering properties in FASCOD.'

Algorithms and technical support will be provided for the calculation of parameters required for the develop- ment of RT models appropriate to GCM calculations. The calculation of statistical and correlated $\mathbf{k}$ distributions, transmission functions, and high-accuracy RT calculations for rapid-RT-model validations will be addressed.

The two-stream rapid-RT algorithm of Toon et al. will be studied and validated with particular emphasis on the spectral overlap problem for different molecular species. The improvement attainable will be studied, and the computational expense of using four streams with a rapid diagonalization procedure will be established.

Support for measurement recommendations to address requirements for RT-model improvements for GCMs will be provided as will support for the analysis and interpretation of measurement results and the incorporation of their results into the models and databases, as appropriate.

RT calculations will be performed with the highaccuracy model in both the short- and longwave region for specific measurement situations chosen by the ARM scientific team. Calculations will be performed with the rapid-RT model on extended measurement sets for validation and model improvement.

A close collaborative effort will be maintained with researchers involved in spectral-radiance and transmittance measurements. Particular emphasis will be placed on spectral cloud properties and on molecular spectroscopic issues, including continuum and heavymolecule effects.

Results to Date: The line-by-line radiative-transfer model LBLRTM has been made fully functional and is available to the ARM scientific community. The CHARTS code, for use in conjunction with LBLRTM for line-by-line multiple scattering calculations, has been validated for the thermal region and is being validated for the solar region. CHARTS is approximately two orders of magnitude faster than previous codes for the same calculation. The availability of these codes enables the line-by-line calculation of radiative transfer in atmospheres including clouds and aerosols. These codes of high accuracy enable the validation of the model physics of atmospheric radiation and provide the necessary inputs for the creation and validation of rapid models for direct application to GCMs.

Refinements have been made to the CKD (Clough, Kneizys and Davies) water-vapor-continuum model, 
which will provide improved agreement with ARM spectral measurements and improved accuracy for GCM calculations. A significant effort has been made in conjunction with Pacific Northwest Laboratory (PNL) to implement an operational Quality-Measurement Experiment to concurrently assess the quality of the AERI spectral-radiance measurements, the LBLRTM radiativetransfer model, and the ARM capability to provide an accurate definition of the atmospheric state.

\section{PARAMETERIZATION OF CONVECTIVE CLOUDS, MESOSCALE CONVECTIVE SYSTEMS, AND CONVECTIVE-GENERATED CLOUDS}

COTTON, WILLIAM B.

\section{COLORADO STATE UNIVERSITY}

$\begin{array}{lr}\text { FY } 1993 & 210 \\ \text { FY } 1992 & 0 \\ \text { FY } 1991 & 192\end{array}$

$11 / 01 / 92-10 / 31 / 93$

Objective: To use a mesoscale cloud model to develop cloud parameterizations for use in GCMs.

Product: Physical understanding and numerical techniques adequate to develop algorithms in GCMs that characterize heat exchange (radiative, latent, and sensible), optical, and other properties of clouds accurately and with computational efficiency.

Approach: A highly capable and documented model will be used to develop cumulus-parameterization schemes that include contributions of both deep and shallow convection and mesoscale vertical velocities; identify criteria that determine if ordinary cumulonimbus or mesoscale convective complexes will dominate for a given set of conditions; examine the feasibility of simulating cirrus clouds created from the outflow of cumulus clouds; and test the parameterization schemes with data, including ARM data.

Results to Date: The first generation of a cumulonimbus draft-core parameterization scheme is operational and has been independently tested.

Explicit (nonparameterized) simulations have been carried out of a midlatitude squall line (June 10-11, 1985), a midlatitude mesoscale convective complex (MCC;
June 3-4, 1985), and a tropical cloud cluster (EMEX-9). All three cases agreed quite well with observations. Analysis code is being written that calculates budget terms and major forcing terms of energy-, heat-, and water-budget equations for the various flow branches of the simulated MCCs.

The small-scale turbulence component of the Weissbluth scheme is now being generalized to simulate middleand high-level clouds formed from the remnants of MCCs.

\section{AREA-REPRESENTATIVE ESTIMATES OF SURFACE HEAT FLUX FOR CART SITES}

COULTER, RICHARD L.

ARGONNE NATIONAL LABORATORY
$\begin{array}{cc}\text { FY } 1993 & 200 \\ \text { FY } 1992 & 200 \\ \text { FY } 1991 & 200 \\ 10 / 01 / 91-09 / 30 / 92\end{array}$

Objective: To develop methods for integrating local and nearly local heat-flux estimates made at CART sites to produce an overall estimate representative of the extended CART-site areas.

Product: Transfer functions for estimating representative surface-heat-flux values for extended CART areas from direct local measurements of surface heat flux and visualizations of larger-scale meteorological features.

Approach: Local surface-heat-flux estimates at many sites within the extended CART domain will be used in combination with area-averaging techniques, such as sodar and light scintiliation, to determine the way in which different categories of surfaces contribute to heat fluxes on a larger scale. These large-scale estimates will be used to estimate still-larger-scale surface heat fluxes that might be inferred from other ARM investigations. Visualization techniques will be used with surface-based remote sensing (lidar, radar, and aircraft) to determine the variability across the CART domain because of large-scale structures characteristic of the planetary boundary layer as a whole.

Results to Date: The study of coherent structures as measures of horizontally integrated surface heat flux was shown to be effective. Using the conditionally sam- 
pled vertical velocity field in thermal plumes as a measured value of the convective velocity scale was shown to relate the surface heat flux with the capping inversion height. Because the plumes have a lifetime of several tens of minutes, they effectively integrate over a large horizontal surface energy input; they also provide the primary mechanism for energy transfer from the surface to the upper troposphere and into cumulus clouds. An analytical model of the expected heat flux sbove the farm as a function of distance from the desert-farm interface was found to agree very well with measured values when the convective velocity scale was used as a measure of the average surface heat flux.

Wavelet transforms have been applied to the data from this field study. This transform has the ability to determine the relative importance of different scales of atmospheric motion to the data, similar to Fourier transform but localized in time scale. Thus, they can be used to isolate and identify coherent processes. This analysis technique is being applied to the above-farm and above-dessert convective-velocity and temperaturevariance data to establish the important scales influencing thermal-plume development.

\section{DEVELOPMENT OF AN INTEGRATED DATA ASSIMILATION/SOUNDING SYSTEM IN SUPPORT OF THE ARM PROGRAM}

\section{DABBERDT, WALTER F. \\ NATIONAL CENTER FOR ATMOSPHERIC RESEARCH}

$\begin{array}{lr}\text { FY } 1993 & 600 \\ \text { FY } 1992 & 0 \\ \text { FY } 1991 & 594\end{array}$

$$
11 / 01 / 92-10 / 31 / 93
$$

Objective: Develop an integrated sounding system (ISS) and data-assimilation system (DAS) to couple in situ and remote-sensing instruments with a state-of-the-art mesoscale model.

Product: A prototype ISS and mesoscale model adapted to DAS application.

Approach: An existing suite of observational tools (including in situ networks, remote-sensing systems, and mobile observation capabilities) that have been recommended by the scientists planning the ARM field experiments will be adapted for ARM. In particular, continuous vertical profiles and some 3-D patterns of wind, temperature, moisture, and important trace gases will be obtained with ground-based remote sensing (radar, lidar, radiometry). A state-of-the-art mesoscale dynamical model will be introduced to assimilate and interpret data output from the measurement system.

Results to Date: A prototype ISS was tested through comparison with in situ measurements taken by instrumented aircraft. This ISS included a $915-\mathrm{MHz}$ wind profiler with interferometric capabilities, a radio acoustic sounding system (RASS), a CLASS sounding system, and an enhanced surface meteorological station. The interferometric profiler derives the three components of the wind from a single transmitted beam of data by the use of multiple receivers. Interferometry has many advantages over conventional Doppler wind profilers, particularly for deriving fluxes, because the assumptions of spatial and temporal homogeneity are relaxed and the sampling time is reduced.

Measurements for future data-assimilation tests were obtained. A recently developed nonhydrostatic version of the NCAR/Pennsylvania State mesoscale model, which includes data assimilation capabilities for a wide variety of measurement platforms, will be used in these tests. The nonhydrostatic capabilities allow for many convective processes to be directly resolved. Efforts with Observing Simulation System Experiments (OSSEs) suggest that a modest network of observing stations (spacings of $\sim 200 \mathrm{~km}$ ) can produce significant improvements in the accuracy of derived meteorological variables, an intercomparison of model-estimated and measured surface fluxes to improve their treatment in numerical models, and the incorporation of a detailed radiation scheme in the model.

\section{RADIATIVE PROPERTIES OF NONUNIFORM CLOUDS}

DAUM, PETER H.; KLEINMAN, L. I.; and STEPHENS, GRAEME L.

BROOKHAVEN NATIONAL LABORATORY and COLORADO STATE UNIVERSITY

$\begin{array}{ll}\text { FY } 1993 & 365 \\ \text { FY } 1992 & 240 \\ \text { FY } 1991 & 240\end{array}$

$10 / 01 / 92-09 / 30 / 93$ 
Objective: To improve the treatment of cloud-radiation interactions and feedbacks in GCMs by focusing on the problem of radiative transfer through clouds in the atmosphere.

Product: New and better predictions of cloud radiative transfer in a way that can be directly incorporated into GCMs and related models.

Approach: This program will combine both theoretical and observational studies of radiative transfer to test current parameterizations of cloud radiative-transfer processes, to assess the effects of cloud inhomogeneities on domain-averaged radiative properties, to evaluate methods of retrieval of cloud droplet-size distributions and liquid-water path from spectral reflectance measurements, to perform radiative-transfer calculations for clouds with nonhomogeneous microphysical and geometric properties, and to develop new methods of modeling radiative transfer through the atmosphere with broken cloudiness.

Results to Date: Data from the Southern Great Plains CART site has been routinely received and analyzed to yield relations between cloud radiative and macrophysical properties. Flux ratio relations have been explored to determine downwelling IR at the surface from more commonly available measurements.

In the area of deterministic radiative transfer, two very different approaches have been aimed at performing calculations in multidimensional media. The first is known as the Fourier-Riccati method, which allows for the radiative-transfer equation in the spectral domain and solves the transfer as an initial-value problem. The second expresses the angular components of the radiance in terms of a spherical harmonic expansion while discretizing the spatial part with finite differences. The resulting system of equations is then solved iteratively. Both of these methods have been used to explore various issues relevant to atmospheric science.

In stochastic radiative transfer, a backward Monte-Carlo method has been developed that uses path-length distributions to calculate the domain-averaged fluxes for arbitrary media.

\section{POINT-AREA RELATIONSHIPS FOR GLOBAL CLIMATE MODELING}

\author{
DORAN, J. CHRISTOPHER
}

\section{PACIFIC NORTHWEST LABORATORY}

$\begin{array}{ll}\text { FY } 1993 & 420 \\ \text { FY } 1992 & 420 \\ \text { FY } 1991 & 420\end{array}$

$10 / 01 / 91-09 / 30 / 92$

Objective: To develop procedures for extrapolating local measurements of radiation and heat flux to larger areas that are representative of a GCM grid cell.

Product: Improved parameterizations of surface heat fluxes that account for subgrid-scale variability arising from inhomogeneous surface properties.

Approach: Sites will be selected that are well-defined and have relatively stationary meteorological conditions and surfaces that will emphasize the importance of subgrid scale variations in fluxes. Interpolationextrapolation methods will be developed to derive areal average flux values from point measurements. These methods will then be applied to specific CART sites. Simultaneously, surface and aircraft data will be collected to examine and improve formulations for GCM-grid-size areas. Mesoscale numerical models will be used as a tool for systematic testing of the effects of surface inhomogeneities.

Results to Date: Processing of the data from the 1992 Boardman field experiment has been begun with an emphasis on examining aspects of boundary layer behavior not measured in the 1991 experiment. The growth of the mixed layer (as seen from potential temperature profiles obtained with airsondes) and the development of a "farm breeze" (suggested by wind profiles obtained from three sodars) were of particular interest because of their possible implications for boundary-layer parameterizations in climate models. A model for estimating sensible- and latent-heat fluxes over agricultural crops was tested and refined with data from the Boardman experiment, and good results were obtained. Such a model should prove useful for extrapolating from point to area values of fluxes over the Southern Great Plains CART site. Numerical simulations of the response of the boundary layer to spatially inhomogeneous surface heat fluxes have begun, 
and the decay of turbulent fluctuations in the mixed layer is being analyzed.

\section{TEMPORARY ASSIGNMENT TO THE COMMITTEE ON EARTH AND ENVIRONMENTAL SCIENCES}

EDGERTON, SYLVIA

ARGONNE NATIONAL LABORATORY

$\begin{array}{lr}\text { FY 1993 } & 150 \\ \text { FY 1992 } & 0 \\ \text { FY 1991 } & 0\end{array}$

$10 / 01 / 92-09 / 30 / 93$

Objective: To provide staff support to the Committee on Earth and Environmental Sciences (CEES).

Product: Services in support of the activities of the CEES.

Approach: Anticipated tasks will include: (1) reviewing national and international R\&D programs in earth sciences dealing with global environmental changes; (2) assisting in the interagency and intergovernmental planning and coordination of $R \& D$ programs in earth sciences; (3) identifying and defining R\&D needs related to earth and environmental sciences; (4) assisting in the preparation of long-range research planning and budget planning documents for the overall national effort in earth and environmental sciences; and (5) providing reviews, analyses, advice, and recommendations for CEES concerning national policies and programs in earth and environmental sciences related to assessing people's impact on the global environment.

\section{STUDY OF LONGWAVE RADIATION CODES FOR CLIMATE STUDIES: VALIDATION WITH ARM OBSERVATIONS AND TESTS IN GENERAL CIRCULATION MODELS}

ELLINGSON, R., and BAER, F.

\section{UNIVERSITY OF MARYLAND}

$\begin{array}{lr}\text { FY } 1993 & 352 \\ \text { FY } 1992 & 0 \\ \text { FY } 1991 & 289\end{array}$

$$
11 / 01 / 92-10 / 31 / 93
$$

Objective: To develop an observationally calibrated, optimum longwave-radiation model for use in GCMs.

Product: An improved longwave-radiative-transport model suitable for implementation in GCMs.

Approach: An optimum longwave-radiation model will be developed for use in GCMs that has been calibrated with state-of-the-art observations; the impact of the longwave radiative forcing will be assessed in a GCM; the sensitivity of a GCM to the radiative model used in it will be determined; and the contribution of the longwave radiative forcing will be determined in comparison with shortwave radiative forcing, sensible heating, thermal advection and expansion.

Results to Date: The ability of LBL radiation models to calculate the downward flux at the surface was determined, and the flux was shown to be about $4 \mathrm{~W} / \mathrm{m}^{2}$ for midlatitude winter conditions. The uncertainties associated with the use of different profiling techniques in calculating the downwelling radiance and flux at the surface were identified. The variability among calculations of the radiation codes from different climate models and their differences relative to clear-sky radiance and flux observations were determined. The uncertainties associated with estimating the effective cloud fraction from surface longwave flux observations were identified. The sensitivity of calculations to different formulations of finite-sized clouds was tested, and the effective cloud fraction was found to be sensitive to cloud shape and aspect ratio but insensitive to the cloud spatial distribution or distribution of cloud area. The sensitivity of models that calculate longwaveradiative heating to variability of input conditions and to the structure of the model algorithms was determined. And the response of GCMs to the variability in heating rates provided by the longwave radiation algorithms was quantified.

\section{JASON STUDY OF SELECTED TOPICS}

ENTZMINGER, JOHN N.

\section{ADVANCED RESEARCH PROJECTS AGENCY}

$\begin{array}{lr}\text { FY } 1993 & 206 \\ \text { FY } 1992 & 0 \\ \text { FY 1991 } & 0\end{array}$

$07 / 15 / 93-07 / 14 / 94$ 
Objective: To bring new scientific expertise, understanding, insights, and critical faculties to the problems faced by the government.

Product: Briefings to the director of DOE's Environmental Sciences Division and to other DOE managers on study results and methods.

Approach: The JASON group will survey and study proposed ways of ameliorating possible future greenhouse warming from increased atmospheric $\mathrm{CO}_{2}$ and will contribute ideas of its own. Among the methods to be considered are increasing the Earth's albedo by the injection of $\mathrm{H}_{2} \mathrm{SO}_{4}$ particulates into the stratosphere, arrays of reflecting balloons, orbiting mylar mirrors, and modification of cloud-droplet seeding. Other techniques to be reviewed involve the removal and disposal of $\mathrm{CO}_{2}$ from the atmosphere or from fossil-fuel burning sources and a limited consideration of certain energy technologies to replace such burning. JASON will also investigate issues related to ARM data collection, processing, and dissemination, including test processing of selected data sets.

\section{EFFECT OF CLOUDINESS HETEROGENEITY ON THE RADIATIVE BUDGET AT THE TOP OF THE ATMOSPHERE AND AT THE SURFACE: MODELING, VERIFICATION, AND ANALYSIS}

\section{GAUTIER, CATHERINE}

\section{UNIVERSITY OF CALIFORNIA AT SANTA BARBARA}

$\begin{array}{lr}\text { FY 1993 } & 170 \\ \text { FY 1992 } & 0 \\ \text { FY 1991 } & 169\end{array}$

$$
11 / 01 / 92-10 / 31 / 93
$$

Objective: To develop new, more realistic methods of modeling the structure of clouds and improved parameterizations of radiative transfer in cloudy skies.

Product: Basic insights on radiative transfer in clouds and potentially improved descriptions of the behavior of clouds and radiative transfer as simulated by GCMs.

Approach: Innovative methods will be developed to describe the structure and optical properties of cloud fields. Cloud scaling characteristics will be determined from data obtained with satellites. The basic multifractal parameters will be estimated. New, high-perfonnance computer algorithms will be investigated for moreefficient extraction of the basic multifractal parameters from observations. Then, clouds with realistic scaling characteristics will be simulated with multifractal analysis and other methods. Anisotropy will be introduced to simulate the vertical stratification and texture variations of clouds. Different sampling and averaging strategies will be evaluated by modeling the temporal evolution of cloud and radiation fields.

Innovative methods will be developed to describe radiative transfer in cloud fields. Mean radiances and radiation fluxes of clouds will be computed as a function of their scaling characteristics. Approaches will vary from simplified methods to Monte Carlo computations. Departures from plane-parallel theory (used in GCMs) as a function of cloud parameters will be evaluated. Modeled radiances and fluxes of clouds will be evaluated by comparison to ARM testbed data, observations from satellites, and data collected by programs complementary to ARM. The results of the investigations will be applied to improve GCM simulations of clouds and radiative transfer.

Results to Date: High-radiometric-quality surface and operational satellite data were collected (particularly over the Antarctic) and analyzed. Preliminary results indicate no dependence between the surface albedo of Antarctic snow and ice and cloud thickness. The analysis of atmospheric spectral (IR) emission suggests that cloud mid-IR emissivity varies spectrally and is regionally specific.

Systematic multifractal analysis of the scaling properties of satellite cloud radiances (covering visible, IR, and microwave lengths and scales from $260 \mathrm{~m}$ to $4000 \mathrm{~km}$ ) were also performed. These analyses confirmed the multifractal nature of these fields and provided the three parameters characterizing their behavior. We developed multifractal models of clouds, performed initial experiments with dynamic multifractal models, laid the foundations for the generalization of cascade processes (Lie cascades), and explored multifractal phase transitions. Radiative transfer through heterogeneous clouds and relationships between cloud and radiation singularities were investigated with different approaches. 


\section{DEVELOPMENT OF A GCM STRATIFORM- CLOUD PARAMETERIZATION}

\author{
GHAN, STEVEN J.
}

\section{PACIFIC NORTHWEST LABORATORY}

$\begin{array}{lr}\text { FY } 1993 & 190 \\ \text { FY } 1992 & 178 \\ \text { FY } 1991 & 0\end{array}$

$10 / 01 / 92-09 / 30 / 93$

Objective: To develop and test a cloud microphysical model that distinguishes cloud water and ice and captures the subgrid distribution of the bulk properties of clouds in GCMs.

Product: A new parameterization to represent the distribution of bulk cloud properties that are most significant to radiation slgorithms in GCMs.

Approach: A bulk-cloud-microphysics model developed for cloud simulations will be adapted for application to the full life-cycle of stratiform clouds. Cloud droplet number will be represented as a prognostic variable that is dependent on aerosol concentrations and vertical motion. Other prognostic variables to be addressed are cloud liquid water, cloud ice, and ice-crystal number density; subgrid-scale distributions of cloud water and ice will be represented. Cloud variables will be parameterized for use in GCMs. The parameterizations will be evaluated in single-column mode with ARM observations and in a GCM. Verification will be achieved through comparison with observed radiative fluxes, cloud distribution, and vertically integrated cloud liquid water.

Results to Date: A parameterization of the droplet nucleation process was developed that expresses the number concentration of droplets formed in terms of aerosol number concentration, vertical velocity, radiative-cooling rate, and aerosol size and composition. Comparisons between the parameterized number and that simulated by a detailed size-resolving nucleation model show agreement to within $50 \%$ for aerosolnumber concentrations ranging from 50 to $5000 / \mathrm{cm}^{3}$ and for vertical velocities ranging from 1 to $500 \mathrm{~cm} / \mathrm{s}$.

We have begun to represent subgrid variations in cloud microphysical processes by expressing subgrid variations of warm clouds in terms of idealized probability density functions (pdfs) of cloud water and rain. The moments of the pdfs are related to the first and second moments of the mass concentrations. The second moment of cloud water is predicted with a second-order turbulence closure scheme for boundary-layer clouds. The second moment of the rain concentration is diagnosed by integrating the autoconversion and collection of cloud water over the joint pdfs of cloud water and rain. The scheme was tested in a single-column model before its application to a GCM.

The cloud parameterization in its simplest form (prescribed droplet number and no subgrid variations) has been applied to the PNL version of CCM1. Preliminary simulations are qualitatively realistic.

\section{DEVELOPMENT AND TESTING OF PARAMETERIZATIONS FOR CONTINENTAL AND TROPICAL ICE-CLOUD MICROPHYS- ICAL AND RADIATIVE PROPERTIES IN GCM AND MESOSCALE MODELS}

\begin{tabular}{|c|c|}
\hline \multicolumn{2}{|c|}{$\begin{array}{l}\text { HEYMSFIELD, ANDREW J. } \\
\text { NATIONAL CENTER FOR ATMOSPHERIC } \\
\text { RESEARCH }\end{array}$} \\
\hline FY 1993 & 156 \\
\hline FY 1992 & 137 \\
\hline FY 1991 & 0 \\
\hline
\end{tabular}

$03 / 01 / 93-02 / 28 / 94$

Objective: To develop a new understanding of the radiative properties of clouds in the middle and upper atmospheres, especially those containing ice.

Product: New parameterizations to address the impact of ice or ice-water clouds on the atmospheric radiation balance as represented in GCMs.

Approach: Microphysical data on convectively produced cirrus and altocumulus gathered during FIRE, TOGA/COARE, the Central Equatorial Pacific Experiment (CEPEX), and ARM will be used with measurements of the clouds' bulk radiative effects to parameterize cloud radiative properties in terns of microphysical composition. Ice-particle spectra and crystal shapes will be correlated to ground-based radar and lidar data. Parameterizations developed with a nonhydrostatic cloud model will be subjected to sensitivity analysis to understand the impact of these clouds on GCM performance. 
Results to Date: Unique in situ cloud microphysical measurements of ice crystal sizes, concentrations, and shapes have been gathered in tropical anvil cirrus. These data are being analyzed to reveal and interpret the properties of cirrus anvils.

A simple life cycle model of anvil cirrus clouds is being used to understand the physical processes that determine the longevity and radiative properties of such clouds. By treating the ice-crystal size spectra discretely and retaining information on ice-crystal shape, we can more accurately determine how crystals of different sizes affect the anvil life through sedimentation and sublimation and can determine radiative properties. High-resolution radiative measurements during TOGA/COARE and CEPEX provide a baseline for inferring the effects of tropical cirrus clouds on radiation and the effect of radiation on cloud microphysics.

The Clark nonhydrostatic, anelastic dynamic cloud model is being initialized with TOGA/COARE data and compared with supporting aircraft dropsonde soundings and in-cloud and rumotely sensed data. CEPEX anvils will also be studied and tested against CEPEX in situ microphysical data. With this model, large tropical anvils are being reproduced to build parameterizations of anvil microphysical properties in terms of environmental conditions and larger-scale dynamics.

\section{CLOUD PARAMETERIZATION IN CLIMATE MODELS}

KAO, C.-Y. JIM

\section{LOS ALAMOS NATIONAL LABORATORY}

$\begin{array}{lr}\text { FY } 1993 & 236 \\ \text { FY } 1992 & 236 \\ \text { FY } 1991 & 0\end{array}$

10/01/92-9/30/93

Objective: To use advanced measurements in the ARM program to investigate improved parameterizations of low-level stratus or stratocumulus, penetrative cumulus, and high-level cirrus clouds in GCMs.

Product: Improved parameterizations of low-level stratus and stratocumulus, penetrative cumulus, and cirrus.
Approach: A cloud-parameterization technique will be developed that can parameterize low-level stratus/stratocumulus, penetrative cumulus, and high-level cirrus with new physical schemes for improving cloud simulations in GCMs. The principal tools will be the physically based Arakawa-Schubert scheme for penetrative clouds and the Sundqvist scheme, which will be refined by the incorporation of diffusion processes derived from a second-orderturbulence approach. Clouds will be more accurately parameterized as both the measurements of ARM and the climate models continue to improve the resolution of temporal and spatial fluctuations. Performance-analysis results will be used to iteratively improve parameterizations.

Results to Date: The Arakawa-Schubert cumulus parameterization scheme for convective clouds and the Sundqvist scheme for nonconvective layered clouds have been incorporated into the Los Alamos GCM. The detrained cloud water simulated by the ArakawaSchubert scheme is further carried into the Sundqvist scheme so that cumulonimbi can serve as the source for the formation of cirrus. Furthermore, the cloud water of nonconvective clouds is subject to transport by both advection and diffusion in a prognostic sense. The clouds in this package are no longer assumed to be black emitters in the longwave radiation. Rather, their emissivities are assumed to depend on cloud water content. Our model results have been compared against the ISCCP satellite data. The agreement includes cloud cover, vertically integrated cloud water, and horizontal distribution of cloudiness. The model cold bias is also remedied with our new cloud package.

A process-level module has also been developed to investigate the diurnal variability of marine low-level stratiform clouds associated with the short- and longwave radiation and boundary-layer turbulence. The diurnal variation of marine boundary-layer clouds was found to be primarily modularized by the shortwave radiation that, in turn, triggers a decoupling of turbulence because of the stabilization by solar heating in the cloud layer. The net effect is the reduction of water-vapor flux from the ocean surface. The model results compare favorably with the FIRE field experiment data. 


\section{INTERFACING BETWEEN A HIERARCHY OF NUMERICAL MODELS IN ARM}

KAO, C. -Y. JIM, and LEONE, JOHN

\section{LOS ALAMOS NATIONAL LABORATORY}

$\begin{array}{ll}\text { FY } 1993 & 200 \\ \text { FY } 1992 & 400 \\ \text { FY } 1991 & 400\end{array}$

$10 / 01 / 92-09 / 30 / 93$

Objective: To establish the relationship between the collective effects of clouds from cloud-scale simulations and the mean variables resolvable in GCMs.

Product: Methods for interfacing and data assimilation among a hierarchy of numerical models.

Approach: A hierarchy of models (ranging from GCMs to mesoscale models to cloud models) will be used to develop methods for data assimilation between the models as well as between models and field observations. The large-scale results of GCM calculations will be used to drive (or guide) mesoscale models that will subsequently provide input to the cloud models. The collective effects of clouds can then be derived from the detailed processes associated with the individual components of the cloud system and, in turn, related to the mean variables in the GCMs. GCM parameterizations can thus be extensively tested against detailed, 3-D process models. The data-assimilation methods will allow ARM measurements, augmented with numerical field experiments, to be incorporated into the models in a dynamically consistent way to increase the reliability and universality of GCM parameterizations.

Results to Date: Using National Meteorological Center (NMC) global analyses to drive the Regional Atmospheric Modeling System (RAMS) model we obtained a monthly climatology over the western United States and conducted several GCM/RAMS interface simulations for the western United States, focusing on extreme wintertime cases.

The ability of the RAMS model to capture the average precipitation and surface temperature over the western United States was clearly demonstrated with an independent data set for comparison. The dependence of cloud formation and precipitation on topography over the complex terrain of the model domain was also simulated, as was the ability of the microphysics parameterization to produce fairly realistic spatial patterns of liquid- and ice-phase precipitation accumulations.

Two regional climate sensitivity experiments designed to test the impact of grid configuration on the prediction of precipitation were conducted. One had an extended western boundary; the other had a nested grid over the Oregon coast region. Both experiments were found to improve the precipitation field by increasing amounts in the Pacific Northwest and reducing the amounts within the intermountain west. We are currently conducting similar simulations for the Southern Great Plains with the nested-grid capability and the ARM Experiment Operations Plan 2 (EOP2) data package.

GCM/RAMS-interface results for the western United States extreme events (such as warm/wet and cold/dry cases) shower that the RAMS mesoscale model is capable of assimilating large-scale characteristics provided by the LANL GCM and of depicting detailed temporal and spatial variations within the model domain.

\section{PASSIVE CLOUD DYNAMICS MEASUREMENT: A FLOW-FIELD- REGISTRATION APPROACH}

KEGELMEYER, W. PHILIP, JR.

\section{SANDIA NATIONAL LABORATORIES}

$\begin{array}{lr}\text { FY } 1993 & 115 \\ \text { FY } 1992 & 115 \\ \text { FY } 1991 & 0\end{array}$

$10 / 01 / 92-09 / 30 / 93$

Objective: To passively measure cloud-bottom heights, cloud geometries, and cloud dynamics with sequential cloud images, a technique that exploits the characteristics of optical flow fields.

Product: Computer-image-processing algorithms suitable for extracting cloud-bottom heights, cloud geometries, and cloud dynamics from cloud images acquired with a whole-sky imager (WSI).

Approach: WSI digital data will be integrated with simultaneously acquired ceilometer data to develop WSI 
sequences for which cloud base-height ground truth is available. A technique will be developed that uses optical flow fields to extract cloud-bottom height, cloud geometries, and cloud dynamics with an algorithm for computing optical flow fields from the cloud images. Cloud-motion and geometry-correction issues necessary to provide a common frame of reference for the flow fields will be addressed. New, multiseasonal, time-dependent, digital cloud data will be acquired and used to investigate the extension of the flow-field and registration algorithms to the measurement of cloud shape, size, and extent.

Results to Date: The core cloud-base-height-extraction algorithm was materially improved by explicitly handling certain common error sources, upgrading the epipolar-line computation with an analytic model, and using a performance analysis tool (POOF) to objectively optimize other control parameters.

The improved algorithm was applied to synthetic data sets representing eight cloud types. The results for the altostratus data set, in which $84 \%$ of the confidently computed heights were within $200 \mathrm{~m}$ of being correct, are typical. It was also tested on real data extracted from two WSIs separated by $5.54 \mathrm{~km}$. Typically, $78 \%$ of the confidently computed heights were within $200 \mathrm{~m}$ of agreeing with a ceilometer.

The cloud-base-height algorithms were extended to implement the determination of fractional cloud cover and a variety of cloud-morphology statistics: the mean and standard deviation of cloud and cloud-free path, cloud area, the number of distinct clouds, cloud perimeter/area ratios, and clear-sky perimeter/area ratios.

\section{A HIERARCHICAL APPROACH TO IMPROVED CLOUD-RADIATION PARAMETERIZATIONS FOR CLIMATE MODELS}

KIEHL, JEFFREY T.

\section{NATIONAL CENTER FOR ATMOSPHERIC RESEARCH}

$\begin{array}{lr}\text { FY } 1993 & 294 \\ \text { FY } 1992 & 301 \\ \text { FY } 1991 & 0\end{array}$

$01 / 15 / 93-01 / 14 / 94$
Objective: To improve the parameterization or representation of radiation algorithms in GCMs with respect to the impact of convective clouds and the range of mass fluxes to be expected.

Product: Improved representations and parameterizations for the NCAR CCM and GFDL GCM of the formation and radiative impact of convective clouds.

Approach: The clear-sky radiation modules used in the NCAR and GFDL GCMs will be validated with a cumulus ensemble model (CEM) that includes the detailed radiative optical properties for ice particles to study convective clouds.

The CEM will be validated by comparing it to ARM data from the Southern Great Plains and Tropical Western Pacific sites. It will then be used to improve the parameterization of the radiative properties of the anvil outflow from the convective core and to improve the parameterization of cumulus mass flux. These new parameterizations will be tested in the most up-to-date versions of the NCAR and GFDL GCMs.

Results to Date: Ice optical properties have been successfully included in the NCAR CCM2, and the sensitivity of the GCM simulation to cloud ice effective particle size has been explored. The upper tropospheric tropical jet structure is quite sensitive to ice-particle size. Consistent ice properties must be included in both the solar and infrared spectral regions. If these properties arc included only in the solar region, the thermal and dynamic response of the tropics is significantly different than if the affects are included in both spectral regions.

These optical properties have also now been included in the resolved convection model being used to study convective systems. The importance of day-night differences in convective activity has been explored. Preliminary results suggest a strong diurnal effect. Nightime conditions seem to promote more convective activity and deeper penetration by that activity. The nighttime and daytime runs employed the same surface temperature.

A new diagnostic parameterization of a cloud's liquid and ice water was developed for the NCAR CCM2 that significantly reduces existing surface-temperature biases in the current version of the GCM. The diagnosed liquid-and ice-water content is derived from the column precipitable water predicted by the model. 


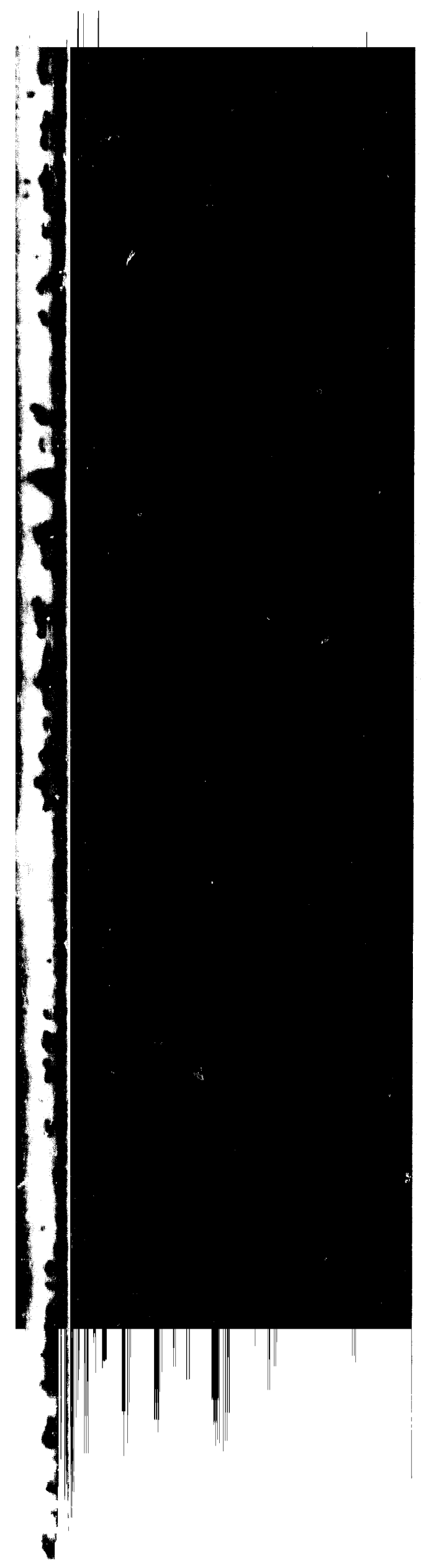

\section{SHIPBOARD MEASUREMENTS OF THE CLOUD-CAPPED MARINE BOUNDARY LAYER DURING FIRE/ASTEX}

\section{KROPFLI, ROBERT A.}

\section{WAVE PROPAGATION} LABORATORY/ERL/NOAA

$\begin{array}{lr}\text { FY } 1993 & 230 \\ \text { FY } 1992 & 292 \\ \text { FY } 1991 & 0\end{array}$

\section{$12 / 01 / 92-11 / 30 / 93$}

Objective: To explore issues relevant to establishing an oceanic ARM Program CART site and to obtain a data set on marine clouds and radiation.

Product: Identification of technical and logistical issues associated with establishing an ocean-based CART site.

Approach: An existing suite of NOAA Wave Propagation Laboratory (WPL) surface-based remote sensors will be deployed on shore and on a ship during the Atlantic Stratocumulus Transition Experiment (ASTEX). The instrument suite will include a Doppler lidar, a Doppler cloud radar, a Doppler wind profiler, a water-vapor/liquid microwave radiometer, and a radio acoustic sounding system (RASS). Measurements will be compared with those acquired with similar island-based sensors operated by Pennsylvania State University, Colorado State University, and NOAA WPL.

Results to Date: Remote and in situ sensors were deployed on the R/V Malcolm Baldridge, and a complete set of remote sensors was deployed on Porto Santo Island during ASTEX. The island-based 8-mmwavelength cloud radar was successful in documenting long-lasting MBL microcells and also in establishing the diurnal variations of in-cloud turbulence. The radar was also used to profile drizzle-drop parameters an!, along with a microwave radiometer, cloud-water-content profiles. Studies of stratus and cirrus cloud-boundary statistics are under way.

Observations from the new compact Doppler lidar used for the first time at Porto Santo are being processed. Measurements of wind profiles and cloud-base structure have been obtained for each io. observation interval. Processing algorithms are being developed and tested to measure vertical-velocity statistics.
Most of the shipboard data has been processed, and preliminary studies have revealed that the diurnal variations of cloud properties in ASTEX were quite different from those observed in the equatorial Pacific region. There, diurnal variations of cloud optical thickness and cloud fraction were negligible; so, variations in system albedo and transmission coefficient were dominated by solar-zenith-angle effects. In ASTEX, the cloud fraction had a substantial diurnal variation with a maximum of about 0.55 at sunrise and a minimum of 0.29 well after sunset, which led to a steady decrease of system optical thickness during the daytime and a skewing of the albedo and transmissioncoefficient curves.

\section{SPECTROSCOPIC STUDY OF $\mathrm{H}_{2} \mathrm{O}$ ABSORPTION IN THE 8- TO 14- $\mathrm{m}$ ATMOSPHERIC WINDOW}

KULP, THOMAS J.

\section{LAWRENCE LIVERMORE NATIONAL LABORATORY}

$\begin{array}{ll}\text { FY } 1993 & 180 \\ \text { FY } 1992 & 180 \\ \text { FY } 1991 & 180\end{array}$

$10 / 01 / 92-09 / 30 / 93$

Objective: To provide an accurate characterization of atmospheric-water-vapor absorption in the infrared transmission window at 8 to $14 \mu \mathrm{m}$.

Product: Data to fill in the gaps in the present empirical database regarding the $8-$ to $14-\mu \mathrm{m}$ line and continuum absorption and data on far-wing line structure that will contribute to an improved theoretical understanding of the water-vapor continuum.

Approach: Diode-laser absorption spectroscopy and Fourier-transform infrared (FTIR) spectroscopy with a long-pathlength absorption cell will be used to make important measurements. The line parameters of as many lines as possible in and around the window region will be quantified at various temperatures and pressures. The measurements will be compared with the results of HITRAN calculations. The dependence of the continuum absorption of water vapor on both temperature and wavelength will be quantified in the window region, and the results will be compared with previous measurements, where possible. The feasibility 
of using double-resonance techniques will be evaluated to study individual, isolated rovibrational lines for the purpose of characterizing the far-wing absorption in water-vapor absorption. Measurements will be performed on molecules that have simpler band structures than water to allow lineshapes to be observed under more-isolated conditions than is possible with water.

Results to Date: The intensities and broadening coefficients of about 20 lines have been measured with the diode-laser spectrometer. FTIR spectroscopy has been used to measure the spectrally resolved transmittance of room-temperature water samples at a resolution of $1 \mathrm{~cm}^{-1}$. The FTIR results were used to determine the self-broadened continuum at room temperature. At the present, no spectrally resolved measurement of the room-temperature continuum is available in the literature. The experimental results were compared with the predictions of the line-by-line radiative-transfer model (LBLRTM) and with a radiative-transfer code developed at NASA/GISS that employs the theoretical continuum of Q. Ma. The results were also compared with the past measurements of Burch and Alt, who measured the self-broadened continuum at selected wavelengths in the window region.

\section{ANALYSIS OF CLOUD RADIATIVE FORCING AND FEEDBACK IN A CLIMATE GCM}

\section{LACIS, ANDREW A.}

\section{GODDARD INSTITUTE FOR SPACE STUDIES}

$\begin{array}{lr}\text { FY } 1993 & 300 \\ \text { FY } 1992 & 0 \\ \text { FY } 1991 & 298 \\ & \\ & 11 / 01 / 92-10 / 31 / 93\end{array}$

Objective: To improve and validate the radiative code of the GISS GCM by intercomparisons with LBL calculations and to improve diagnostic analyses and treatment of clouds in the GCM, including their interaction with dynamics and radiation.

Product: Improved radiative-transfer codes and model diagnostics that permit more-detailed analyses of climate-feedback interactions and that facilitate model comparisons to satellite data.
Approach: A new cumulus and stratiform cloud parameterization will be installed in the GISS GCM. Based on a cloud liquid- and ice-water budget, the new scheme will be made fully interactive with the model's solar and thermal-radiation calculations, including dependence on cloud particle size and optical depth. GCM diagnostics will be upgraded to facilitate intercomparisons with other GCMs and observational data.

Results to Date: The solar-radiation code of the GCM has been improved by parameterizing the single-Gausspoint (SGP) doubling-adding algorithm to accurately reproduce multiple scattering by clouds and aerosols as functions of particle size, optical depth, and solar-zenith angle. The magnitude and latitudinal dependence of climate feedbacks caused by water vapor, clouds, surface albedo, and advected-energy transports is obtained from annually averaged GCM output with a 2-D radiative-convective-advective equilibrium model (2-D RCAM). AMIP simulations with the new cloud scheme are now able to reproduce the El Niño highcloud signature, while the old scheme could not.

\section{LASER REMOTE SENSING OF WATER VAPOR}

LAPP, M.; MELFI, S.; and GOLDSMITH, J.

\section{SANDIA NATIONAL LABORATORIES and GODDARD SPACE FLIGHT CENTER}

$$
\begin{array}{lr}
\text { FY 1993 } & 250 \\
\text { FY 1992 } & 650 \\
\text { FY 1991 } & 451 \\
& \\
10 / 01 / 91-09 / 30 / 92
\end{array}
$$

Objective: To extend Raman lidar technology to enable vertical profiles of water vapor to be obtained both day and night.

Product: A prototype Raman lidar system with sufficient accuracy, reliability, and automation for inclusion in CART.

Approach: The detailed characteristics needed for a day/night-capable water-vapor probe at a CART site will be identified and defined. A complete optimization analysis and instrument-performance model will be developed, including selection of the optimum laser wavelength. The daytime probe upgrades will be 
designed and fabricated. Research and development will be performed on a Raman shifter for changing the wavelength of the candidate laser source, determining absorption cross sections for potential beam-absorbing gases more accurately, potential laser-induced fluorescence interference, and user-friendly dataprocessing software. The daytime-probe upgrades will be integrated into an existing Raman lidar system. The model will be field tested and validated. And the conceptual design and costing of a CART-ready system will be performed.

Results to Date: Simultaneous nighttime measurements of water-vapor profiles have been performed with two collocated, advanced Raman lidar systems. The agreement between the two independent systems provides a striking example of the capability of the Raman-lidar technique.

Field measurements were performed to test two concepts (solar-blind operation and narrowband, narrowfield-of-view operation) for extending the range of daytime Raman-lidar operation.

A conceptual design for a lidar system to be implemented at the Southern Great Plains site was developed. In addition to providing vertical profiles of water vapor, the system will also provide quantitative cloud characterization (base height and, for optically thin clouds, top height and vertical profiles of scattering ratio), depolarization ratio (useful for identifying particle phase), measurements of aerosol scattering ratio, atmospheric density (useful for profiling temperature), and backscatter signals at three wavelengths (to provide particle size information in the submicron regime).

\section{ARM SUPPORT \\ LEIFER, ROBERT \\ ENVIRONMENTAL MEASUREMENTS LABORATORY}

$\begin{array}{lll}\text { FY } 1993 & 415 \\ \text { FY 1992 } & 415 \\ \text { FY 1991 } & 415\end{array}$

$10 / 01 / 92-09 / 30 / 93$

Objective: To provide surface aerosol a.d ozone observations at the Southern Great Plains CART site for use in radiative models and in automated back-trajectory analyses to characterize air-mass identification.

Product: A set of complementary instruments to characterize the surface-aerosol and ozone concentrations.

Approach: Procurement documents will be written for equipment, an aerosol-sampling manifold will be designed and constructed, a fully automated computer program will be developed to provide at least twice daily an air-mass back-trajectory calculation, and a unique characterization of the air mass (rural, urban, marine, etc.) will be performed in real time.

Results to Date: The following instruments were approved by ARM and ordered: aerosol scattering, integrating nephelometer, aerosol optical absorption, filter technique, aerosol size distribution, optical particle counter, aerosol number concentration, aerosol particle counter, ozone concentration, and ultraviolet absorption. All but the aerosol scattering and absorption instruments have been received and tested.

An aerosol sampling manifold has been designed and approved by ARM for installation at the Southern Great Plains site. The manifold is under construction at EML. Working drawings for mounting the $10-\mathrm{m}$ sampling inlet to the aerosol trailer have been submitted to ARM, and construction should soon be completed.

EML has established computer linkage with Brookhaven National Laboratory (New York) and the U.S. Navy Fleet Numerical Oceanography Center (California) to obtain real-time global meteorological data for use in forecasting pollutant transport. This capability has resulted in the development of a 3-D trajectory model, based on operational wind forecasts produced by the National Meteorological Center's grid model. Trajeciories are obtained twice a day and are presently being stored in EML's computer. These files will be transferred to the ARM Experimental Center. Based on a given trajectory, air-mass characterization (rural, urban, etc.) and not the actual trajectory is needed by modelers. Preliminary work has been started to automate the air-mass characterization. 


\section{TESTBED MODEL AND DATA ASSIMILATION FOR ARM}

LOUIS, JEAN-FRANCOIS

ATMOSPHERIC AND ENVIRONMENTAL
RESEARCH, INC.

$\begin{array}{lr}\text { FY } 1993 & 170 \\ \text { FY } 1992 & 0 \\ \text { FY } 1991 & 163\end{array}$

$11 / 01 / 92-10 / 31 / 93$

Objective: To develop methods of data assimilation on a scale consistent with climate models and to design and implement a model for testing parameterizations of cloud characteristics and radiative-transfer processes.

Product: Methods to effectively test parameterizations suitable for use in GCMs.

Approach: A 1-D model, the AER Local Forecast Adaptation (ALFA) model, will be further de eloped to simulate conditions at ARM testbed sites in the same manner as does a GCM over a single grid cell. The 1-D model will be constructed so researchers can test interactive modules that contain the necessary parameterizations (e.g., on radiative transfer, cloud microphysics, and the dynamics of the atmospheric boundary layer, the lower $2 \mathrm{~km}$ of the atmosphere).

The ARM data, together with analysis results from the National Meteorological Center, will be assimilated with the ALFA model to produce a continuous description of the atmosphere above the ARM site. The adjoint method will be used to ensure that differences between observations and the evolving variables computed with ALFA are minimized.

The means to conduct sensitivity studies on parameters important in GCM modules will be developed. A form of the adjoint method will be used for this purpose. As a test case, the sensitivity of the Toon-Ackerman radiation model to various cloud parameters will be evaluated.

Results to Date: A sophisticated radiation scheme with the capability of simulating partial cloudiness has been included in the single-column model. The adjoint of the model, including the complete physical package, has been developed and allows an efficient computation of the gradient of the simulation output with respect to all the inputs. Tests of the variational data assimilation system have been started. A picture of the evolution of the atmosphere over the CART site that is close to the observed data (e.g., standard deviation smaller than $1 \mathrm{~K}$ for surface temperature) can now be obtained with the smoothness expected from the scale of a climate model. Tests of the magnitude of the smoothness constraint are in progress. The model also computes surface fluxes and other quantities that can be compared to observations. These will eventually be used in the data assimilation system. The sensitivity of the model forecasts to the optical parameters of clouds is currently being computed.

\section{GROUND-BASED MILLIMETER-WAVE CLOUD-PROFILING RADAR SYSTEM}

McINTOSH, ROBERT E.

\section{UNIVERSITY OF MASSACHUSETTS}

$\begin{array}{lr}\text { FY } 1993 & 279 \\ \text { FY } 1992 & 0 \\ \text { FY } 1991 & 319\end{array}$

$$
11 / 01 / 92-10 / 31 / 93
$$

Objective: To develop a millimeter-wavelength radar to fill the recognized need for cloud-field mapping.

Product: A remote-sensing system that can yield information on the penetration-backscatter strength tradeoff, the water-ice ratios of cloud water substance, profiles of cloud water amount, backscatter/absorption ratios, and vertical velocities in clouds.

Approach: A millimeter-wavelength radar will be developed for cloud-field mapping. It will use two wellselected wavelengths to help distinguish between scattering and absorption processes. Measurements of the polarization of the return signal will assist the interpretation of water to ice ratios, and the Doppler shift in the return will indicate vertical velocity, a crucial quantity for cloud formation. Existing prototype and operational airborne millimeter-wave designs will be used as a basis for this high-power ground-based mobile facility.

Results to Date: The cloud-profiling radar system (CPRS) was assembled and tested at the Southern Great Plains CART site. Preliminary measurements of 
rain-bearing stratiform clouds have shown the ability to detect multiple cloud layers to a height exceeding $10 \mathrm{~km}$ at $33 \mathrm{GHz}$; all layers but the highest were detected at 95 GHz. Significant depolarization was detected at the melting band ( $4 \mathrm{~km}$ in altitude) at both 33 and $95 \mathrm{GHz}$, and vertical velocity was measured at both 33 and 95 GHz. The dual-polarization, dual-frequency antenna; construction and testing of the radar systems (33 and 95 $\mathrm{GHz}$; integration and testing of the VXI-based dataacquisition system; and purchasing and customizing the mobile laboratory were completed. The data-processing algorithms are currently being upgraded to compute all of the important polarimetric parameters in real time, and schemes to perform polarimetric calibrations in the field are being developed.

\section{DEVELOPMENT OF ROTATING SHADOWBAND SPECTRAL RADIOMETERS AND GCM-RADIATION-CODE TEST DATA SETS}

\section{STATE UNIVERSITY OF NEW YORK AT ALBANY}

MICHALSKY, J., and HARRISON, L.
$\begin{array}{cr} & \\ \text { FY } 1993 & 250 \\ \text { FY } 1992 & 0 \\ \text { FY } 1991 & 249\end{array}$

$11 / 01 / 92-10 / 31 / 93$

Objective: To develop a high-quality data set of cloudor aerosol-modified direct and diffuse solar irradiances that would allow development of improved GCM parameterizations, a filter-based rotating shadowband radiometer (RSR), and a charged coupled device (CCD) array based RSR.

Product: A database of ground and satellite data related to direct and diffuse solar irradiances and prototype RSR instrumentation.

Approach: Existing ground-based radiation and meteorological data sets of high quality that can be used in conjunction with existing satellite data will be assembled to provide modelers with a basic data set fo: refining the cloud/radiation models in current GCM codes. An observational program will be carried out to add to the database key parameters measured at a single site in upstate New York.
Two variants of the RSR will be developed, tested, and deployed to measure the spectrally resolved direct and diffuse irradiances. One of these instruments will measure the irradiance in seven discrete spectral intervals of $10 \mathrm{~nm}$ (or greater) balf-width selected within the range 380 to $2500 \mathrm{~nm}$; the other will measure the irradiance in 256 wavelength intervals nonlinearly distributed over the range 350 to $1050 \mathrm{~nm}$, providing a spectral resolution of $0.6 \mathrm{~nm}$ at the blue end of this spectrum and $10 \mathrm{~nm}$ at the infrared end. The first instrument is intended to fill the needs of the Extended Observing Network of each CART, and the second is proposed for the Central Site.

Results to Date: Two years of radiation and meteorological data from Albany, New York, have been combined with upper-air data from Albany County Airport for selected days to test GCM radiation codes. Besides total shortwave and longwave radiation, we collected spectral direct and diffuse data with our multifilter rotating shadowband radiometer (MFRSR) at six wavelengths and calculated column aerosol and water vapor. We have recently installed an all-sky scanning radiometer to measure cloud cover every 5 $\min$.

The MFRSR has been accepted as a standard instrument for the ARM program and will be installed at all extended and auxiliary stations in the Southern Great Plains CART site. The data acquisition for the solar and infrared observing system at each of these sites will be the MFRSR logger. A new 32-channel printed circuit board has been delivered to accommodate all SIROS measurements. MFRSR technology has been transferred to a commercial vendor. Data-reduction algorithms have been developed to automatically and objectively calculate aerosol optical depth, to improve the shortwave measurements made with the silicon sensor in the MFRSR, and to retrieve column water vapor. The rotating shadowband spectroradiatometer field prototype is complete and being tested and calibrated. 


\section{DEVELOPMENT OF AN SWIR SOLAR SPECTRAL RADIOMETER FOR ARM}

\author{
MURCRAY, DAVID G. \\ UNIVERSITY OF DENVER
}

$\begin{array}{lr}\text { FY } 1993 & 137 \\ \text { FY } 1992 & 98 \\ \text { FY } 1991 & 0\end{array}$

$12 / 01 / 92-11 / 30 / 93$

Objective: To develop and test a highly accurate, absolutely calibrated, short-wavelength infrared (SWIR) spectral radiometer capable of measuring solar radiance in the 0.8 - to $5-\mu \mathrm{m}$ spectral interval.

Product: A prototype FTIR radiometer system suitable for the acquisition of sky radiances in the $0.8-$ to $5-\mu \mathrm{m}$ spectral region.

Approach: An existing University of Denver moderate-resolution FTIR interferometer system will be modified to obtain data in the short-wavelength region. A high-temperature (>1500 K) black-body-radiance source will be developed, and a National Institute of Standards and Technology (NIST)-referenced radiance source calibrated from 0.8 to $6 \mu \mathrm{m}$ will be purchased. The interferometer 's'stem will be calibrated with the high-temperature black-body source and the NISTreferenced source. Observations will be made with this system on a number of clear days, and modified Langley plots will be constructed from these observations to determine the absolute spectral radiance of the sun in the spectral interval from 0.8 to $5 \mu \mathrm{m}$. The onsite data will be regularly referenced to the blackbody source and periodically against the NIST-referenced standard. Based on these studies, one or more short-wavelength, moderate-resolution solar interferometers will be constructed to measure the incoming direct solar radiation for the CART site.

Results to Date: Initial tests with an available commercial interferometer indicated that excellent instrument stability was practical (in the 2.5 to $15 \mu \mathrm{m}$ region). Because a very stable instrument would need only occasional calibration, the black-body-source may not be necessary and has been delayed. A moderateresolution interferometer was purchased for the 0.8- to $7-\mu \mathrm{m}$ interval, and special optics have been installed.
The optical design permits viewing the tungsten-ribbon calibration standard with the identical components used to view the center of the sun. The new system has been tested with a cooled germanium detector ( 0.9 to 1.6 $\mu \mathrm{m})$. The system can produce a spectrum from the calibration source with $\mathrm{S} / \mathrm{N}$ of greater than 300 in less than $4 \mathrm{~min}$ and from the Sun in a few seconds. Stability of the system is good: less than $1 \%$ change in a week. Preliminary tests with the InSb detector (used for 1.5 to $5 \mu \mathrm{m})$ will also be completed.

\section{PARTIAL FUNDING OF THE INTERNATIONAL WORKSHOP ON RADIATIVE TRANSFER AND CLOUD PHYSICS IN GCMS \\ OHRING, GEORGE \\ NATIONAL OCEANIC AND ATMOSPHERIC ADMINISTRATION}

$\begin{array}{lr}\text { FY } 1993 & 10 \\ \text { FY } 1992 & 0 \\ \text { FY } 1991 & 0\end{array}$

$02 / 01 / 93-01 / 31 / 94$

Objective: To advance the treatment of clouds and their radiative effects on GCMs.

Product: Published monograph of presentations and discussions.

Approach: A workshop will be conducted that will be jointly fi "ded by NOAA, NASA, the World Climate Research s'rogram (WCRP), and DOE.

\section{A PRECISE, PASSIVE, NARROW-BEAM FILTER, INFRARED RADIOMETER AND ITS USE WITH LIDAR IN THE ARM PROGRAM}

$$
\text { PLATT, C. M. R. }
$$

COMMONWEALTH SCIENTIFIC AND INDUSTRIAL RESEARCH ORGANIZATION

$\begin{array}{lr}\text { FY } 1993 & 80 \\ \text { FY } 1992 & 60 \\ \text { FY } 1991 & 0\end{array}$

$12 / 01 / 92-11 / 30 / 93$ 
Objective: To upgrade existing radiometric instrumentation, to implement the LIdar-RADiometer (LIRAD) method at the ARM Southern Great Plains CART site, and to use it to develop improved parameterizations of cloud radiative effects for incorporation in GCMs.

Product: A prototype high-precision filter radiometer suitable for measuring cloud infrared radiances in the 8to $13-\mu \mathrm{m}$ spectral region.

Approach: An infrared filter radiometer will be constructed with sufficient sensitivity and precision to measure low radiances from thin cirrus clouds at high altitude as well as higher radiances from lower and denser clouds against a background of water-vapor emission. The radiometer will be tested and demonstrated at the ARM Pilot Radiation Observation Experiment (PROBE) at Kavieng, Papua New Guinea, to acquire accurate and precise observations of tropicalcirrus-cloud-downwelling infrared radiance in combination with laser-radar (LIDAR) backscatter observations. With simultaneously measured temperature and watervapor profiles (e.g., with a balloon-borne sonde), accurate values for the cloud emittance and optical depth will be calculated at wavelengths in the atmospheric window between 8 and $13 \mu \mathrm{m}$. Correlations will be established between cloud albedo and cloud infrared radiative properties for parameterization algorithms.

Results to Date: The radiometer was designed, fabricated, and tested by the CSIRO Division of Atmospheric Research. The radiometer has three spectral bands at 8.62,10.86, and $12.04 \mu \mathrm{m}$, each with a bandwidth of about $1 \mu \mathrm{m}$ and a variable field of view of from 3 to 30 milliradians. It operates with a liquidnitrogen-cooled mercury cadmium telluride detector.

Observations of tropical cirrus and midlevel clouds in PROBE were taken with lidar and the new infrared radiometer. Initial results indicated the ubiquity of cirrus layers in that region. At times, these layers were more than $6 \mathrm{~km}$ in depth; on several days, the cirrus exhibited a diurnal cycle, with the densest cirrus occurring around midday (and unconnected on those days with thunderstorm activity). Thin layers of midlevel altocumulus were quite a common feature in association with both cirrus and cumulus clouds. The data will be analyzed in association with other water-vapor, liquid-water, radiosonde, and interferometric data obtained by U.S. scientists in the PROBE experiments.
ARM TROPICAL PACIFIC EXPERIMENT (ATPEX): ROLE OF CLOUD, WATER-VAPOR, AND CONVECTION FEEDBACKS IN THE COUPLED OCEAN/ATMOSPHERE SYSTEM

RAMANATHAN, V., and BARNETT, T.

\section{SCRIPPS INSTITUTION OF OCEANOGRAPHY}

$\begin{array}{ll}\text { FY } 1993 & 662 \\ \text { FY } 1992 & 662 \\ \text { FY } 1991 & 528\end{array}$

08/01/93-07/31/94

Objective: To observe and model the radiative feedbacks and energy fluxes in the tropical western Pacific and use this understanding to advance climatemodel development.

Product: An increased understanding of the enhanced greenhouse effect and the role of clouds in modifying the effect.

Approach: Data-processing and imaging techniques will be developed to combine ARM data with satellite data. The work will be closely coordinated with the ARM Science Team to ensure that the satellite data and the ARM data complement each other as well as provide the data essential for testing the hypothesis of the super greenhouse effect in the tropical western Pacific. The satellite data will include atmospheric profiles of temperature and humidity, estimates of total water vapor, and 3-D radiative information for cloud research.

A coupled ocean/atmosphere GCM will be used to seek understanding of the measurements and to assist in selecting the elements to be measured. The model will be used to assist in validating and advancing the cloudradiation parameterizations used in GCMs with emphasis on the rcle of tropical convection. The seasonalcycle and the ENSO data (including the satellite data) will be used in conjunction with the model. The hypothesis is that a "super" greenhouse must be induced by the unique energy balance, with the clouds regulating the sea-surface temperature (SST) yet owing their existence to the warm SSTs and the convection.

State-of-the-art, 3-D visualization will be used in both tasks to aid understanding and to facilitate the comparison of the data with the model information. 
Results to Date: The Central Equatorial Pacific Experiment (CEPEX) was successfully completed, the fundamental objective of which was to test the thermostat hypothesis of SST regulation. CEPEX deployed four aircraft and a ship in conjunction with satellite and buoy measurements to measure the vertical structure of the water-vapor super greenhouse effect, the effect of cirrus on radiation fluxes over the equatorial Pacific, the east-west gradients of SST, and the evaporative flux from the sea surface.

Preliminary results confirm that in regions of deep convection water vapor leads to a super greenhouse effect over the warmest oceans in the equatorial Pacific and that extended cloudiness resulting from convection leads to a large reduction in solar radiation over the warm oceans. Direct radiometric observations also reveal that the effect of cirrus clouds on longwave radiation at the surface is significantly smaller than the effect on solar radiation. Parallel studies with the University of Hamburg GCM confirm the significant role of cirrus as an elevated heat source within the upper troposphere. The GCM study shows that longwave heating caused by the warm-pool cirrus drives the Walker circulation and thus warms the entire tropical troposphere.

\section{USE OF ARM DATA TO TEST AN IMPROVED PARAMETERIZATION OF UPPER TROPOSPHERIC CLOUDS FOR USE IN CLIMATE MODELS}

RANDALL, DAVID A.

\section{COLORADO STATE UNIVERSITY}

$\begin{array}{lr}\text { FY } 1993 & 166 \\ \text { FY } 1992 & 164 \\ \text { FY 1991 } & 0\end{array}$

$$
12 / 01 / 92-11 / 30 / 93
$$

Objective: To improve parameterizations of uppertropospheric cloudiness and to determine the effect on the atmospheric energy balance by the distribution of such clouds.

Product: Improved parameterizations for upper tropospheric cloudiness for the 3-D CSU GCM.

Approach: Three fundamental models and modeling approaches will be exploited, namely the Colorado State
University (CSU) GCM, a 1-D version of that GCM, and the UCLA cumulus ensemble model. Data acquired by the ARM program at the field measurement sites will be used to drive the UCLA 1-D models; predictions of those models will be compared directly to later observations to evaluate the performance of the models. This approach will use existing models to address (1) a prognostic approach for the parameterization of cloud formation and dissipation, including grid-cell average cloud water and its relation to the fractional cloud amount and (2) the development and testing of improved diagnostics for the parameterization of cloud formation and dissipation, including the refinement of current semiempirical correlations for stratiform and convective clouds as functions of relative humidity and cumulus mass flux respectively.

Results to Date: The cumulus ensemble model has been upgraded with a radiation parameterization and improved parameterizations of cloud microphysics and turbulence. Numerous lengthy simulations have been performed. A new method has been devised to partition the simulated cloud fields into convective and stratiform cloudiness. Sensitivity of the cloud-formation process to cloud-radiation interactions has been investigated. The EOP3 data from the Oklahoma ARM site has been used to investigate the observed fluctuations of the convective available potential energy. The 1-D GCM has been driven with GATE data in preparation for driving it with suitable ARM data. A workshop on the simulation of cloud and radiation fields at the ARM sites has been organized.

\section{HIGH-SPECTRAL-RESOLUTION RADIANCE MEASUREMENTS}

$$
\text { REVERCOMB, } \mathrm{H} \text {. }
$$

\section{UNIVERSITY OF WISCONSIN}

$$
\begin{array}{lr}
\text { FY } 1993 & 475 \\
\text { FY } 1992 & 0 \\
\text { FY } 1991 & 519 \\
& \\
\multicolumn{1}{r}{11 / 01 / 92-10 / 31 / 93}
\end{array}
$$

Objective: To design and deliver three instruments to provide highly accurate observations of the emitted atmospheric radiation and of atmospheric transmission.

Product: Fourier transform infrared (FTIR) spectroscopic expertise and instrumentation to support 
the improvement of high-resolution LBL radiativetransport codes and to improve the physical understanding of longwave radiation transport in the atmosphere.

Approach: State-of-the-art FTIR radiometric instrumentation will be developed to acquire high-quality, highspectral-resolution, infrared, atmospheric-radiance data and atmospheric molecular-absorption data, especially in the water-vapor-continuum region. The data acquired with this instrumentation will be compared with the predictions of infrared-radiation-transport models, such as FASCOD3, to identify candidate areas for code improvements (e.g., by implementing improved or alternative spectral-line parameters).

Three instrument types will provide the highly accurate observations needed for detailed comparisons with LBL calculations and for determining the radiative characteristics of clouds, aerosols, and trace gases. Two (AERI and AERI-X) will make highly accurate measurements of the atmospheric emitted radiation, and the third (SORTI) will measure the atmospheric transmission to high accuracy with observations of the sun at different air masses.

The AERI (atmospheric-emitted-radiance interferometer) instrument will operate over the spectral range of 4 to $20 \mu \mathrm{m}$ (2500 to 500 wavenumbers) at a resolution of 1 wavenumber. It will be sufficiently inexpensive to be deployed throughout the ARM networks and will provide the link between point measurements and the larger scale. A system of AERI instruments would also provide important 3-D measurements of meteorological parameters in the lower atmosphere.

The AERI-X instrument will provide improved spectral resolution ( 0.1 wavenumber) over the wavelength range from 4 to $20 \mu \mathrm{m}$. It will provide the higher spectral resolution needed at a more limited number of locations to acquire the best available emission observations for comparison with LBL calculations.

The solar radiance transmission interferometer (SORTI) measuremenis with a resolution of 0.002 wavenumber will yield the atmospheric transmission at essentially full resolution. It will reveal deficiencies in the way model calculations handle absorption line shapes and line interactions.

Results to Date: The AERI prototype has been operated successfully at the Southern Great Plains (SGP) CART site in the June 1993 intensive operational period (IOP), with data released to the Science Team in near real time. The software systems developed for automatic, stand-alone operation were successfully demonstrated. The SORTI has successfully collected data at the University of Denver and is ready for tests at CART. Good agreement was achieved between the SORTI and a similar instrument operated simultaneously.

\section{CLOUD AND AEROSOL CHARACTERIZATION FOR THE ARM CENTRAL FACILITY: MULTIPLE REMOTE-SENSOR TECHNIQUES}

\section{SASSEN, KENNETH}

\section{UNIVERSITY OF UTAH}

$\begin{array}{lr}\text { FY } 1993 & 91 \\ \text { FY } 1992 & 0 \\ \text { FY } 1991 & 184\end{array}$

$$
11 / 01 / 92-10 / 31 / 93
$$

Objective: To develop and deploy a combination of active and passive remote-sensing systems to observe features of clouds and to develop empirical and cloud model-based algorithms for the remote-sensing characterization of the cloud atmosphere.

Product: Measurements and interpretation with a combination of instruments including polarizationdiversity lidar, 8-mm radar, IR and visible radiometers, and an all-sky video imager.

Approach: A polarization-diversity lidar (PDL) will be built that will use two wavelengths with parallel and orthogonal polarization components to enhance observation of cloud distribution and type, cloud structure, cloud ice/water ratio, crystal shape and orientation, and cloud optical properties. The system plus two supporting, passive, remote-sensing systems (an infrared radiometer and an all-sky video imaging system) will be deployed in conjunction with NOAA equipment in a field experiment to compare multiple sensing-system findings.

Results to Date: Four-channel motion control has been added to our polarization-diversity lidar (PDL), which is now capable of simultaneous two-color $(0.532$ and $1.06 \mu \mathrm{m})$, four-polarization channel, azimuth- and elevation-angle scanning operations at $5^{\circ}$ per second, using the full $10-\mathrm{Hz}$ pulse-repetition rate of the laser 
transmitter. Alternatively, the field-of-view (FOV) of the receivers for each color can be opened and closed in 1-s intervals to study the effects on lidar returns of cloudparticle multiple scattering through an FOV range of $\mathbf{0 . 2}$ to 3.8 milliradians.

The cloud microphysical models have been adapted to simulate the backscattering, multiple scattering, and extinction properties of realistic clouds to help evaluate the information contents of various multiple remotesensor techniques that use lidar and radar and to analyze actual field datasets. These numerical experiments have successfully been supplied to evaluating the variable FOV lidar technique for cirrus-cloud-particle sizing, investigating the relationship between radar reflectivity and ice and water cloud mass contents, and devising an autonomous computer algorithm to detect supercooled liquid clouds embedded in winter mountain storm systems.

\section{WIDELY DEPLOYABLE LOW-COST RADIOMETER FOR THE ARM EXTENDED OBSERVING STATIONS}

SIMPSON, M. L., and SPRATLIN, T. L. OAK RIDGE NATIONAL LABORATORY

$\begin{array}{ll}\text { FY } 1993 & 100 \\ \text { FY } 1992 & 318 \\ \text { FY } 1991 & 400\end{array}$

$$
10 / 01 / 92-09 / 30 / 93
$$

Objective: To design, develop, and demonstrate a customized, pointing, shortwave/near-infrared radiometer and related mathematical techniques to infer concentrations of atmospheric gaseous constituents and sizes of suspended particulate matter.

Product: A field-tested prototype radiometer and associated mathematical techniques for extracting atmospheric parameters of interest.

Approach: A multifunction instrument will be developed that will operate in four modes: as a solartracking radiometer, as a solar-aureole radiometer, as a sky-radiance radiometer, and as a zenith radiometer. Its target design specifications will include:

- Pointing accuracy: 0.1 angular degree or better in two axes
- Field of view: 1 angular degree

- Wavelength range: 0.4 to $2.2 \mu \mathrm{m}$ in eight noncontiguous bands

- Stray-light rejection: $10^{6}$ for sources outside the field of view

- Measurement speed: 146 readings in 3 minutes for the all-sky scan mode

Additional design constraints imposed by the intended service include a hermetically sealed optical system, a temperature-controlled environment for the photodetectors and other sensitive electronic components, userfriendly communications between the instrument and the experimenters, and a ruggedized packaging design suitable for extended field use with minimal maintenance.

Results to Date: A pointing shortwave/near-IR radiometer capable of making solar extinction and aureole observations at CART sites was developed, fabricated, and tested in the laboratory. (Only limited field tests have been performed to date.) In conjunction with an appropriate motorized mount, the new radiometer can track the Sun's disk or perform aureole measurements and can measure sky radiance and cloud passage at the zenith.

To complement and support the pointing radiometer, analytical methods for recovering high-resolution spectral information, atmospheric-constituent concentrations, and particle sizes from measurements of solar radiation in the visible and near-UV wavelength regions were also developed and tested.

The radiometer has been sent to Los Alamos National Laboratory for further field testing.

\section{ARM FOURIER-TRANSFORM- SPECTROMETER DATA-ANALYSIS TOOLS}

$$
\text { SMITH, WILLIAM L. }
$$

\section{UNIVERSITY OF WISCONSIN}

$\begin{array}{lr}\text { FY } 1993 & 190 \\ \text { FY } 1992 & 191 \\ \text { FY } 1991 & 0\end{array}$

$$
12 / 15 / 92-12 / 14 / 93
$$

Objective: To develop and test radiative-ransfer models through the analysis of the spectral-radiance and solarabsorption data. 
Product: Data-analysis tools specifically tailored to retrieve various atmospheric parameters (including cloud optical properties) and temperature and moisture profiles from observational data.

Approach: Radiative-transfer algorithms will be developed and tested to derive atmospheric-state parameters from FTIR observations. Through the comparison of FTIR observations and theoretical calculations (with the use of FASCODE and the HITRAN data base), techniques will be developed for inferring atmospheric temperature and moisture profiles, trace-gas information, and cloud radiative properties from data acquired with the AERI, AERI-X, and SORTI.

Results to Date: Algorithms for the analysis of groundbased FTS observations of atmospheric radiation have been developed for routine application to data from the Southern Great Plains CART site. The algorithms enable the retrieval of planetary-boundary-layer temperature and moisture profiles, cloud-base altitude, cloud-water phase, and cloud microphysical properties (effective particle size and water path), needed for calculations of downwelling radiative fluxes.

Clear-sky radiative-transfer comparisons of LBL FASCODE3P/HITRAN92 calculations have been performed with SGP CART data. Software is being installed at PNL for the retrieval of radiatively active atmospheric variables and for the comparison of radiative-transfer calculations with AERI observations as part of the routine production of SGP scientific data sets.

\section{DIAGNOSTIC MODELING OF THE ARM EXPERIMENTAL CONFIGURATION}

SOMERVILLE, RICHARD C. J.

\section{SCRIPPS INSTITUTION OF OCEANOGRAPHY}

$$
\begin{aligned}
& \text { FY } 1993 \\
& \text { FY } 1992 \\
& \text { FY } 1991 \\
& 11 / 01 / 92-10 / 31 / 93
\end{aligned}
$$$$
119
$$

Objective: To test parameterizations of cloud formation that are currently in use in GCMs with ARM data.

Product: $A$ better understanding of the validity of various parameterizations in use in GCMs.
Approach: A 1-D diagnostic model of a single GCM grid will be used, and measurements of initial conditions and horizontal transports made at the ARM site will be combined with the model to predict cloud formation and characterization. The prediction of clouds by the model will use the parameterizations in use in GCMs. The resulting predictions will be compared with the ARM measurements to test the accuracy of the cloud parameterizations. The relative merits of a variety of competing parameterizations will be studied. Thus, the research will pursue

- Improvements and alterations to the existing onedimensional diagnostic model

- Diagnostic modeling, wherein ARM data and the predictions of various parameterization schemes are compared

- Provision of this new knowledge to GCM modelers who will be able to thereby improve the cloudmodeling aspects of the GCM.

Results to Date: The single-column diagnostic model has been extensively developed to incorporate state-of-the-art physical parameterizations. At the same time, the model was recoded for generality, efficiency, and modularity. The model can now incorporate observational data with arbitrary temporal resolution. Sensitivity studies with different vertical resolutions have demonstrated that typical GCM vertical resolutions inaccurately compute radiative-flux divergences.

Our software was ported to a high-speed Unix workstation. Our graphics routines and data-management procedures have been revised to cope with the heterogeneous nature of ARM data. The cumulus convection scheme developed by $K$. Emanuel and the treatment of cloud optical properties adopted by the second-generation GCM of the Canadian Climate Centre have been incorporated and tested.

We have ollaborated with a group from Science Applications International Corporation to implement a statisuical treatment of cloud radiative properties within the model. 


\section{USGCRP PROGRAM ASSOCIATE}

SPAETH, WARREN T.

\section{PACIFIC NORTHWEST LABORATORY}

$\begin{array}{lr}\text { FY } 1993 & 95 \\ \text { FY } 1992 & 0 \\ \text { FY } 1991 & 0\end{array}$

$12 / 01 / 92-09 / 30 / 93$

Objective: To provide a Program Associate within the U.S. Global Change Research Program (USGCRP) Office to coordinate and support the Process Research Working Group activities.

Product: Program coordination, staff support, and various reports and analyses.

Approach: Staff support will be provided for the Subcommittee on Global Change Research and for the Process Research Working Group. Information exchange and communication among the Subcommittee on Global Change Research Working Groups, the Subcommittee, and participating agencies will be facilitiated. A liaison among the Subcommittee, the USGCRP, the National Academy of Sciences, and relevant international globalchange organizations and programs will be provided. The development of annual budget/program documents required by the Office of Management and Budget (OMB); Committee on Earth and Environmental Sciences; Federal Coordinating Council for Science, Engineering, and Technology (FCCSET); and Congress will be supported. The development and review of the USGCRP special analyses, reports, and other documents requested by the Subcommittee, CEES, FCCSET, OMB, and Congress will be supported. A point of contact will be provided for information on the USGCRP and the Subcommittee on Global Change Research, including providing oversight on behalf of the Subcommittee for the Global Change Research Information Office to be established pursuant to P.L. 101-606.

\section{CIRRUS AND AEROSOL PROFILOMETER FOR RADIOMETRIC MEASUREMENTS}

\author{
SPINHIRNE, JAMES
}

NASA GODDARD SPACE FLIGHT CENTER

$\begin{array}{lr}\text { FY } 1993 & 35 \\ \text { FY } 1992 & 60 \\ \text { FY } 1991 & 0\end{array}$

$12 / 01 / 92-11 / 30 / 93$

Objective: To use a micropulse lidar (MPL) to develop a small, eye-safe, autonomous, laser remote-sensing system with sufficient sensitivity for detecting and profiling cirrus clouds.

Product: A prototype MPL suitable for sustained, unattended ceilometry measurements of cirrus clouds.

Approach: A prototype MPL with a high-pulse-rate (10-kHz) diode-pumped Nd:YLF laser source and a germanium Geiger-mode avalanche photodiode (GAPD) detector will be assembled. The prototype will use a 0.2-nm-width spectral filter and a 0.1-milliradian field-of-view to limit interference of solar background. Photon-counting detection methods will be used in the system. Data acquisition, averaging, and storage will be performed by a PC-compatible computer with a single-card photon-counting system. In cooperation with Pennsylvania State University, the initial MPL system will be tested at the ARM-sponsored Pilot Radiation Observation Experiment (PROBE) in the tropical western Pacific. Following PROBE, the MPL will be demonstrated and evaluated at the Southern Great Plains CART site.

Results to Date: A field-deployable micropulse lidar instrument was completed in October 1992. The instrument was shipped to Kavieng, Paupua New Guinea, and set up for use at the PROBE site during the TOGA/COARE project. It operated successfully for cloud and aerosol profiling through to the end of February. The data showed profiling of all clouds, including thin cirrus and stratospheric aerosols. The instrument was returned, and additional testing and improvements of the signal performance were implemented. A system is in place at the CART site to begin full-time-operation tests. 


\section{ATMOSPHERIC RADIATION MEASUREMENT PROGRAM}

STOKES, GERALD M.

PACIFIC NORTHWEST LABORATORY

$\begin{array}{lr}\text { FY } 1993 & 13750 \\ \text { FY } 1992 & 13888 \\ \text { FY } 1991 & 2764\end{array}$

$10 / 01 / 92-09 / 30 / 93$

Objective: To improve the performance of GCMs of the atmosphere in predicting global and regional climate, focusing specifically on the treatment of radiative-transfer and related models in GCMs under clear-sky, generally overcast, and broken-cloud conditions and the parameterization of cloud properties, cloud formation, and cloud maintenance in GCMs.

\section{Task 1: ARM Program Management}

Gerald Stokes, Pacific Northwest Laboratory, \$1476K; Marvin Dickerson, Lawrence Livermore National Laboratory, \$45K; Sumner Barr, Los Alamos National Laboratory, \$45K; Stephen Schwartz, Brookhaven National Laboratory, \$145K; Robert Charlson, University of Washington, \$25K; Warren Wiscombe, Goddard Space Flight Center, $\$ 99 \mathrm{~K}$; V. E. Zuev, Institute for Atmospheric Optics, Russia, \$50K; Peter Lamb, University of Oklahoma, $\$ 255 \mathrm{~K}$; Thomas Ackerman, The Pennsylvania State University, \$183K; Knut Stamnes, University of Alaska, \$196K; and Sethu Raman, North Carolina State University, \$154K.

Product: The management of the ARM Program, coordination of Science Team activity, and the development of the experimental infrastructure of ARM, known as the Cloud and Radiation Testbed (CART), to provide a basis for the detailed testing and verification of the modeling of subgrid processes in GCMs; the ability to rapidly deploy experiments to the field; and a strong coupling of the scientific goals of the program.

Approach: The ARM Program will be managed from the ARM Project Office, located at PNL, and will consist of three distinct entities: the Science Team, the Instrument Development Program, and the Cloud and Radiation Testbed. The Project office will provide central planning and management for the ARM Program, support of DOE/OHER Environmental
Sciences Division activities, management of specific Science Team efforts requested by DOE, and coordination of ARM Program activities with related efforts and programs in other agencies and countries.

The Science Team will conduct approved research projects; will provide basic guidance for the preparation of sites, the selection and deployment of instruments, and common modeling activities; and will participate in setting the scientific direction of the ARM Program. The site scientists will be integral members of the Science Team and will provide for onsite scientific expertise, will manage a site-related educational program, and will conduct their own research programs. An Adjunct Science Team status will be established whereby scientists funded from other sources can propose a research program to ARM and, if it is accepted, receive ARM data and participate as a member of the ARM Science Team.

The Instrument Development Program (IDP) will provide for the long-term, high-risk development of technologies that will be of long-term interest to ARM and will provide a directed instrument-development activity when an instrument must be developed to meet a need within ARM that cannot be met by other means.

CART will be the basic experimental infrastructure for the ARM Program. CART itself will consist of five planned permanent observing sites; will have an ability to support intensive observations and campaign activities; and will maintain a data system that acquires data from CART-site instruments as well as supplementary data from outside sources, processes that data, archives appropriate data, and provides requested data to individual members of the science Team.

Field sites will be established and managed by site program managers selected from within the DOE national laboratories. Directly supporting this activity as well as the Science Team will be interlaboratory functional teams headed by a principal investigator from one of the DOE national laboratories. These teams will address program needs relative to instruments, data management, Science Team experiment support, site operations, and campaign or offsite user coordination.

Results to Date: Science Team investigators began receiving data in mid-1992 from the first field site in the Southern Great Plains. That site was dedicated in November 1992 to the memory of Fred Luther, an early advocate of an ARM-like measurement program, by the 
DOE Director for Energy Research. Research results from the Science Team were a focus of a special session of the 1993 annual meeting of the American Meteorological Society. The third Science Team meeting was held and focused on the data from the first CART site. The third DOE solicitation for grant applications, with an emphasis on water-vapor measurements, resulted in the acceptance of 37 proposals for FY 1994 funding. Additionally, several Adjunct Science Team Proposals were accepted for support, and they include both foreign and U.S. scientists.

A siting strategy for the second permanent site was developed, and planning was begun for siting decisions and instrument deployment to the second permanent site late in 1994. Data communication from this remote site remains a primary concern and the subject of continuing study. The Science Team Executive Committee has reviewed and commented on the siting strategy and has proven to be an effective means of tapping the expertise of the Science Team in furdamental project planning and decision-making.

Within the infrastructure of the ARM Program, the functional-team approach remains an effective means of addressing the needs of the program and for integrating the expertise and efforts of individuals and groups within the program.

The Instrument Team, in its primary role of selecting, siting and checking out the performance of all CART instruments, has been paying particular attention to the need for quality checks on individual instruments and data streams. The Experiment Support Team is the focal point of the interface between the infrastructure and the Science Team as users of the data (i.e., customers). Feedback from the Science Team through the Experiment Support Team has been an important factor in instrument quality control and in identifying unanticipated observational needs.

The ad hoc working group on aerosol measurement requirements produced a recommended observational program for a CART site and a hallmark report detailing the state of the science in accounting for aerosol influences in the atmospheric radiation balance. Coordination and planning continued for the use of new observational platforms, such as unmanned air vehicles (UAVs), to provide measurements not available by other means. A cooperative program with the DOE UAV program is being pursued to demonstrate the feasibility of UAVs.

\section{Subtask 1(a): Instrument Development Program}

Jeffrey Griffin, Pacific Northwest Laboratory, \$174K; Sumner Barr, Los Alamos National Laboratory, \$45K; and Ken Gage and Ed Westwater, NOAA/Aeronomy and Wave Propagation Laboratories, \$176K.

Product: Management of DOE-funded development of (1) field-hardened instrument designs suitable for replication and installation at CART sites as costeffective, turn-key instruments and (2) more complex instruments used on a campaign basis.

Approach: Instrument-development projects will be funded for 2 to 3 years to (1) verify the atmosphericmeasurement concept, (2) upgrade existing instrumentation to better meet ARM measurement requirements, and (3) deliver field-hardened instrumentation for permanent installation at a CART site.

Results to Date: Currently, 15 instrument-development projects are in progress. Instrumentation and measurement techniques under development include laser radar, millimeter-wave radar, high-resolution atmospheric radiometers, solar radiometers, integrated sounding systems, and data-assimilation methods.

Two IDP-developed instruments are in operation at the ARM Program Southern Great Plains (SGP) CART site: the multifilter rotating shadowband radiometer (MFRSR) and the atmospheric-emitted-radiance interferometer (AERI). They acquire solar and atmospheric radiance/irradiance data, respectively, to aid the improvement of radiative-transport models of the Earth's atmosphere. Two more IDP instruments, the AERI-X and the solar-radiance-transmittance instrument (SORTI) are being added to the SGP CART instrument suite to acquire atmospheric spectra of higher resolution.

These spectra are crucial to detailed studies of LBL radiative-transport models currently being evaluated in the ARM Program. Performancc specifications are being prepared for the procurement of CART-operational systems of three remote sensors (Raman LIDAR for watervapor profiling, polarization-diversity LIDAR, and the cloud-profiling radar system) and their permanent installation at the SGP CART site.

Task 2: Site Implementation and Operations

Douglas Sisterson, Argonne National Laboratory, \$1406K; Sumner Barr, Los Alamos National 
Laboratory, \$831K; Gerald Stokes, Pacific Northwest Laboratory, \$494K; Bernard Zak, Sandia National Laboratories, \$267K; Wayne Manges, Oak Ridge National Laboratory, \$51K; Stephen Schwartz, Brookhaven National Laboratory, \$428K; and Margo Ackley, NOAA/Forecast Systems Laboratory, $\$ 600 \mathrm{~K}$.

Product: Implementation and operational management of the Southern Great Plains site, initiation of site facility and instrumentation planning for the Tropical Western Pacific Ocean and North Slope of Alaska sites, and conceptual planning for operations in the Eastern Ocean Margins (e.g., northeastern Pacific or Atlantic Oceans) and Gulf Stream locales.

Approach: Site program managers will plan and direct the implementation and will manage each CART site with support from the Site Operations Team. The Site Operations Team will assist in the development of the initial site-operations plan for each site, train operations personnel as appropriate, and assist the site operator and the site program manager during initial operations.

Results to Date: The Southern Great Plains site became operational in a phased deployment during FY 1993. The central facility at Lamont, Okla., produced data for Science Team analysis beginning in June 1992. As additional instrumentation was acquired, instruments were deployed and phased into operational service. Extended facilities locations were selected, and instruments and equipment have been deployed for about $80 \%$ of the projected network.

Intensive operational periods (IOPs) during which enhanced rates or types of observations are conducted, often in conjunction with a collaborating measurement program, provided innovative data sets as well as experience hosting visiting scientists and instrumentation deployed temporarily to the site.

A scientifically meaningful data stream was achieved and maintained from the site to the Experiment Center. This achievement was, in part, the result of the successful development of a site infrastructure to support a secure working environment for as many as 25 to 30 scientific and technical personnel. The physical plant now includes temporary buildings for offices, shops, and laboratories; utilities; roadways and grounds; and instrument platforms.

Site implementation and operations are taking significantly different shapes at each of the other four sites. Activities and results common to all, however, include definition of the primary scientific purpose for each site and for preliminary field trials or pilot programs. Conceptual designs for CART sites in oceanic, coastal, and arctic settings have been developed. Because of the remoteness and environmental hostility of the distant sites, the concept of a self-contained, modular facility that can be performance tested prior to deployment and operated remotely for long periods of time has been given great consideration. In the Tropical Western Pacific locale, this facility takes the form of an atmospheric radiation and cloud station (ARCS). An ARCS can be fabricated and tested at home in the presence of optimal support logistics, shipped as a sealed container, and conveniently deployed by a few people, with the shipping container providing a controlled environment for sensitive dataacquisition equipment and instruments. Site selection has progressed to specific recommended locations for the Tropical Western Pacific, North Slope of Alaska, and Eastern Ocean Margin sites. These recommendations are or will be reviewed by the Science Team prior to specific location or instrumentation decisions. Site-analysis documents are periodically updated, with a finalized version published shortly before site deployment.

In the Tropical Western Pacific, the ARM Program's Pilot Radiation Observation Experiment in Papua New Guinea supported the World Climate Research Program TOGA-COARE field measurement program from November 1992 through February 1993. The site program management team gained experience in the harsh tropical environment, established contacts with many of the local officials, and gained first-hand exposure to logistical problems and cultural sensitivities in the region. Alaskan site visits by the program manager of the North Slope of Alaska site led to an in situ demonstration of some project equipment in the arctic environment, where environmental issues, cold temperatures, and permafrost pose very special difficulties. In general, site program managers are encouraged by the skill level and cooperation of their potential hosts while acknowledging the realities of remoteness, harsh environment, and the need to communicate effectively across cultural differences.

\section{Task 3: Experiment and Algorithm Development}

Marvin Dickerson, Lawrence Livermore National Laboratory, \$849K; Gerald Stokes, Pacific Northwest Laboratory, \$528K; Brooks Martner, NOAA Wave 
Propagation Laboratory, \$61K; Gail Anderson, Air Force Philips Laboratory, $\$ 100 \mathrm{~K}$; and Patrick Minnis, NASA Langely Research Center, $\$ 100 \mathrm{~K}$.

Product: Science Team experiment designs for each individual Science Team member that explicitly define measurement requirements; implementations of newly developed or existing algorithms to produce measurements for the Science Team members; and implementations of experiments in the Experiment Center to provide concurrent data-quality checks on two or more data streams.

Approach: The Experiment Support Team will identify and develop procedures to meet the measurement needs of the Science Team. Based on four general measurement strategies that encompass and describe the scientific basis of the various Science Team projects, procedures, including specific algorithms, will be produced for each requested measurement. These fundamental algorithms will be implemented in the Experiment Center at PNL and will be used to derive measurements requested by the Science Team that have a specified space and time resolution. An Experiment Support Team member will meet with each of the principal investigators to define specific measurement requirements for their experiments. In addition, the Experiment Support Team will be responsible for developing the necessary measurement algorithms and quality-measurement experiments that compare two or more measurement data streams. The Experiment Support Team will develop experiment designs for IOPs when extraordinary observations are taken for relatively short periods of time.

Results to Date: Most of the current 45 Science Team members have been visited by the Experiment Support Team, and about 35 experiment designs have been drafted. About 60 CART observations and measurements are currently being delivered to Science Team members with additional data coming online routinely. Observations and measurements external to CART (e.g., NWS satellite and Nested-Grid Model data) are also available to Science Team members through the Experiment Center. A structure for the qualitymeasurement experiments has been developed, and several candidate experiments have been implemented.

\section{Task 4: Instrument Selection/Implementation}

Marvin Wesley, Argonne National Laboratory, $\$ 374 \mathrm{~K}$; Gerald Stokes, Pacific Northwest Laboratory, \$621K;
Roland Hulstrom, National Renewable Energy Laboratory, \$139K; John DeLuisi, NOAA/Climate Monitoring and Diagnostics Laboratory, \$93K; and Larry Thurne, Sandia National Laboratory, \$20K.

Product: Management and execution of ARM infrastructure activities concerning instrumentation and the identification, installation, and implementation of instrument systems.

Approach: Argonne National Laboratory will lead an interlaboratory instrument team that will identify and evaluate technologies for possible incorporation into CART observation systems, will develop the initial operational and calibration protocols for each equipment item before transferring it to individual ARM site operators, and will provide technical information to support the acquisition of instruments.

Results to Date: The ARM/CART Instrument Team continued in the selection, acquisition, and implementation of instrument systems for the SGP CART site and planning for future CART sites. Instrument Team members assigned to mentor specific instrument systems worked with PNL to write technical specifications, to review proposals, to work with selected vendors to ensure meeting CART requirements, to coordinate implementation, to participate in instrument-operation training, and to take responsibility for fully implementing CART instruments for use by the site operator. In addition, the instrument mentors were required to provide data quality control by examining the data acquired and to recommend when data could be released to the Science Team. Extensive coordination with the ARM/CART Data Management Team and the Experiment Support Team was required.

Systems implemented at the SGP CART site central facility by the end of FY 1993 were primarily in support of radiative-transfer studies and included an infrared interferometer, a $915-\mathrm{MHz}$ and a $50-\mathrm{MHz}$ radar wind profiler and radio acoustic sounding system, a $60-\mathrm{m}$ tower instrumented with temperature and humidity sensors, a lidar cloud ceilometer, a whole-sky imaging system, and a vertically pointing infrared thermometer. Systems implemented at extended facilities for large-scale modeling included eight additional energybalance Bowen-ratio surface-flux stations, five surface meteorological-observation stations, and five sets of broadband solar and infrared radiometers and multifilter rotating shadowband radiometers. Approximately 23 extended facilities are planned; one extended facility 
with these three components was installed at the central facility. Three boundary facilities for obtaining information essential for single-column modeling were equipped with balloon-borne sounding systems and passive microwave radiometers, which previously had been implemented at the central facility. Preparations were made to implement radiometric calibration facilities and aerosol instrumentation at the central facility.

Preliminary technical specifications were also produced for some of the instruments intended for the Tropical Western Pacific CART site.

\section{Task 5: Data System Development and Operation}

Ronald Melton, Pacific Northwest Laboratory, $\$ 1560 \mathrm{~K}$; Robert Baker, Argonne National Laboratory, \$534K; Joyce Tichler, Brookhaven National Laboratory, \$223K; Donna Edwards, Sandia National Laboratories, \$20K; Paul Kanciruk, Oak Ridge National Laboratory, \$918K; Lawrence Rothman, Air Force Philips Laboratory, $\$ 110 \mathrm{~K}$; and Cindy Mueller, National Center for Atmospheric Research, \$95K.

Product: Management and operation of the ARM infrastructure activities associated with the design, development, implementation, and deployment of an integrated data system for the acquisition, quality assessment, processing, distribution, and archiving of ARM data.

Approach: A multilaboratory team will design the system from the top down. The team will implement the system incrementally, starting from existing software, where possible. The incremental implementation and deployment will allow the system to be adapted to changing requirements as the CART sites are developed and operated.

Results to Date: Incremental implementation and deployment of the data system continued in parallel with operations. Data products are being delivered regularly to ARM investigators.

The functionality of the site data system grew with the implementation of an instrument-control module that manages the interaction with the different instruments. The ability to communicate with multiple instruments at CART extended facilities has been designed and is being implemented with code-operated data switches. Communications with the boundary facilities is being implemented with high-speed modems and point-topoint communications. The site data system is being incrementally improved as additional capabilities are developed and as problems are encountered.

The ARM Experiment Center started the year as a manual operation. Over the course of the year, tools have been developed that automate some of the manual procedures. This effort will continue.

The ARM archive also started as a manual operation and will be automated over the next several years. Data can be retrieved for use in the ARM Experiment Center or by members of the ARM Instrument Team. A prototype user interface has been designed and is under development. Procurenent of the hardware and software for the long-term archive is proceeding, and the move to the new system will take place in early FY 1994.

\section{Task 6: Campaign Planning and Coordination}

Stephen Schwartz, Brookhaven National Laboratory, \$45K; Dave Renne, National Renewable Energy Laboratory, \$100K; and Ed Westwater, NOAA/Wave Propagation Laboratory, $\$ 120 \mathrm{~K}$.

Product: Management of campaign identification and coordination activities within the infrastructure of ARM, identification of potential relationships between ARM and other experimental programs, and coordination of campaign activity.

Approach: Campaign planning and coordination will be a centralized activity within the program and will be embodied in the ARM Campaign Committee. This committee will act as the primary point of contact for requests for ARM participation in possible campaigns. Additionally, the committee will periodically review CART observational data and assess whether or not a targeted campaign may be necessary to meet specific ARM research objectives. In FY 1994, this process will evolve to embody user-facility-type requests for access to ARM sites to satisfy specified research objectives.

Results to Date: ARM policies and procedures regarding campaigns and scientific activities involving interactions with other agencies and non-ARM investigators have been drafted and are being reviewed for implementation.

Possible campaigns are suggested by an informal proposal to ARM, outlining the nature of the proposed 
activity and the expected scientific results. The typical duration of a campaign is expected to be between 2 weeks and 2 months; campaigns may be one-time or repeated, as appropriate to their scientific objectives. Campaigns may take place at permanent or supplementary CART sites (i.e., other programs taking advantage of activities, observations, and/or measurements at ARM sites) or at other locations, perhaps in conjunction with activities by other programs (i.e., ARM's taking advantage of activities, observations, and/or measurements conducted under the auspices of another entity). A cooperative activity is a more formal interaction with another agency or program that may consist of data exchanges and/or additional observational or measurement activities in support of mutual objectives. Pilot programs are precursor, campaign-like projects conducted in collaboration with other field programs.

The Pilot Radiation Observation Experiment (PROBE) conducted in conjunction with the TOGA-COARE field program in late 1992 and early 1993 in the tropical western Pacific locale was highly successful, giving the program experience in conducting a measurement operation in the Tropical Western Pacific Ocean area. A planned campaign at the Southern Great Plains site will be a proof-of-concept demonstration of the measurement of radiation and atmospheric variables from an unmanned aircraft vehicle.

\section{PARAMETERIZATION OF GCM SUBGRID NONPRECIPITATING CUMULUS AND STRATOCUMULUS CLOUDS WITH STOCHASTIC/PHENOMENOLOGICAL METHODS}

STULL, ROLAND B.

\section{UNIVERSITY OF WISCONSIN}

$\begin{array}{lr}\text { FY } 1993 & 110 \\ \text { FY } 1992 & 66 \\ \text { FY } 1991 & 0\end{array}$

$12 / 01 / 92-11 / 30 / 93$

Objective: To investigate the use of statistical distributions of boundary-layer characteristics to describe and parameterize the phenomenological evolution of cumulus and stratocumulus clouds.
Product: A 1-D boundary-layer and subgrid cloud parameterization for incorporation into 1-D GCMs.

Approach: A mix of deterministic and statistical models will provide parameterizations of boundary-layer clouds for GCMs. The statistical processes will be useable with models other than the 1-D generalmeasurement strategy to be used in the ARM program. With data from previous field programs, the statistical distribution of boundary-layer turbulence and depth, as a function of stability and moisture, will be determined, and parameterizations will be developed. These parameterizations will include surface heating and evaporation based on the surface radiation budget, shading by individual clouds, and land-use patterns. Boundary-layer-depth parameterizations will include convergence and divergence in the boundary layer. Statistical cloud fields produced by the boundary-layer parameterizations will be allowed to feed back into the evolution of the model boundary layer.

Results to Date: Surface-layer data acquired by instrumented aircraft during the Hydrologic Atmospheric Pilot Experiment (HAPEX) field experiment in southwest France shows that temperature and moisture in rising thermals are negatively correlated. Thus, while some thermals are warmer and can rise higher to better trigger clouds, their lifting condensation level (LCL, the altitude needed to form clouds) is also higher, making cloud formation less likely.

The balance between these two opposing influences is measured by plotting the frequency distribution of LCL height vs buoyancy for small segments of air (i.e., air parcels) in the surface layer and superimposing on this graph the mean sounding of buoyancy. The fraction of the surface-layer air parcels lying to the right of the sounding gives the fraction of sky expected to be covered by clouds. Using the aircraft data as a form of calibration, we are developing parameterized frequency distributions as a function of land use that are easy to incorporate in GCMs. The resulting parameterization for boundary-layer clouds are being tested with routine CART data. 


\section{MODELING CLOUDS AND RADIATION FOR DEVELOPING PARAMETERIZATIONS FOR GENERAL CIRCULATION MODELS}

\author{
TOON, O. B., and WESTPHAL, D. \\ NASA AMES RESEARCH CENTER \\ FY 1993 \\ FY 1992 \\ FY 1991 \\ 0 \\ 209
}

Objective: To develop practical parameterizations for treating cirrus and stratus clouds for use in global-scale models and new, accurate treatments of radiative transfer and to apply these parameterizations in a 3-D mesoscale model.

Product: A new paraneterization for treating cirrus and stratus clouds in a GCM and more accurate treatment for radiative transfer.

Approach: A hierarchy of models will be developed, and the accuracy of the radiation model and the cloud model will be tested.

A 4-D data-assimilation analysis package will be developed and used with the mesoscale model to investigate intensive observational studies at ARM sites and to test the ability of dynamical models to properly reproduce moisture fields. The mesoscale model will be used with different cloud microphysics parameterizations and compared with observations at the ARM sites to test the cloud parameterizations.

Radiative-transfer schemes will be improved by developing absorption coefficients and other fast radiation-transfer techniques. The radiation models will be tesied against ARM data, and new absorption coefficients will be derived as needed. Radiation codes will be developed to compare the mesoscale model output with intensity observations and to assess the effects of broken-cloud fields at the scale of both the mesoscale model and the GCM grid scale.

Cloud microphysical parameterizations appropriate for use in GCMs will be developed for cirrus and status clouds. These parameterizations will reflect how the albedos and latent-heat release of marine stratus vary in response to changing environmental conditions, the

properties of tropospheric cirrus clouds, the development of vertical variations in the size distribution of ice crystals, the bulk fall speeds of ice, the effects of the optical and microphysical properties of ice crystals on the generation of cirrus, the sensitivity of cirrus radiative properties to the large-scale dynamical properties in the atmosphere, and the minimum resolution of the size distribution needed to match observations. The cloud microphysical parameterizations in the mesoscale model will be tested by comparing the model results to measurements at ARM sites.

Results to Date: Our 1-D cirrus studies have shown that the clouds are not very sensitive to the number of condensation nuclei present. 3-D mesoscale simulations of cirrus clouds have been compared with radar, lidar, satellite, and other observations of water-vapor and cloud fields and show that the model accurately predicts the characteristics of a cirrus-cloud system. The 1-D stratus model has been used to show the response of marine clouds to changing levels of marine aerosols: the cloud albedo is very sensitive to the numbers of aerosols present but is not bistable, as some researchers have suggested. We have also found that clouds can destroy acrosols in the boundary layer until the clouds thetnselves dissipate and trigger a collapse of the marine boundary layer. We have investigated the singlescattering properties of ice crystals in cirrus clouds. Finally, we have continued to develop radiative-transfer techniques.

\section{USE OF CI.OUD OBSERVATIONS AND MESOSCALE METEOROLOGICAL MODELS TO EVALUATE AND IMPROVE CLOUD PARAMETERIZATIONS}

WALCEK, CHRIS J.

\section{STATE UNIVERSITY OF NEW YORK AT ALBANY}

$\begin{array}{lr}\text { FY } 1993 & 100 \\ \text { FY } 1992 & 79 \\ \text { FY } 1991 & 0\end{array}$

$$
12 / 01 / 92-11 / 30 / 93
$$

Objective: To identify relationships between fractional cloud coverage and various meteorological parameters, to determine the radiative and dynamic impact of fractional cloud coverage, and to parameterize these relationships and impacts. 
Product: Improved cloud-coverage-parameterization techniques for use in GCMs.

Approach: High-resolution output from a mesoscale meteorological model and digitized satellite cloudcoverage data will be analyzed and correlated. Larger-scale meteorological parameters that correlate well with cloud cover will be identified and quantitatively defined; these may include coupled relationships that involve several meteorological parameters, such as relative humidity and stability. Various time and space scales will be examined to extend the mesoscale analyses to larger-scale model applications. A comprehensive mesoscale model will be developed and used to assess the interaction of cloud-scale processes with the larger-scale meteorological environment. Model performance in reproducing larger-scale meteorological processes from this approach will be evaluated against earlier models in which cloud-cover data were used.

Results to Date: Cloud observations have been compared with standard meteorological measurements over the eastern United States. Positive correlations were found between cloud cover and relative humidity and large-scale vertical velocity, and weak negative correlations were found between cloud cover and wind shear and temperature lapse rate. Cloud cover appears to decrease exponentially as relative humidity falls below $100 \%$. The middle troposphere contains the highest cloud amounts at the lowest relative humidities, with mean cloud coverage of $30 \%$ near $50 \%$ humidity at 650 mb. Cloud cover is maximum near the top of the planetary boundary layer, where it is also most sensitive to small changes in relative humidity. A 2 to $3 \%$ change in relative humidity is currently correlated with a $15 \%$ change in cloud cover in the 1000-10-900-mb layer. Most cloud-cover parameterizations used by climate models specify cloud amounts less than those reported by these observations, especially in the middle troposphere, where zero cloud amounts are assumed at relative humidities below 50 to $80 \%$. Thus, current climate simulations may misrepresent the true relationship between cloud coverage and relative humidity and may incorrectly specify any changes in cloud coverage that can result from small changes in relative humidity.

\section{TREATMENT OF CLOUD RADIATIVE EFFECTS IN GENERAL CIRCULATION MODELS}

\author{
WANG, WEI-CHYUNG
}

\section{STATE UNIVERSITY OF NEW YORK AT ALBANY}

$\begin{array}{lr}\text { FY 1993 } & 350 \\ \text { FY 1992 } & 0 \\ \text { FY 1991 } & 349\end{array}$

$11 / 01 / 92-10 / 31 / 93$

Objective: To improve GCM parameterizations of the radiative effects of the vertical overlapping of clouds, to incorporate the cloud optical properties into radiation codes, and to evaluate effects of these improvements on climate simulations.

Product: Improved large-scale atmospheric models, evaluation of parameterizations of clouds and radiativetransfer processes, and specification of measurements and parameterizations that might substantially improve their performance.

Approach: The treatment of clouds in current largescale atmospheric models will be evaluated and further developed. The effects of clouds overlapping horizontally at different heights and variations in their optical properties will be investigated with the use of ground-and satellite-based measurements. The adequacy of random cloud overlapping currently assumed in all GCMs will be evaluated by comparison of results with those from alternative schemes that might be more realistic. The cloud optical parameters of singlescattering albedo, the asymmetry factor, and the extinction coefficient as a function of wavelength will be precalculated by cloud-particle models for use in GCMs or will be derived from ubservations from satellites. Radiation codes in large-scale models will then be modified to use these cloud optical parameters.

The effects of the new treatments of clouds on climate simulations will be evaluated. These simulations will be carried out with a 1-D radiative-convective model and a 2-D radiative-dynamical model to evaluate the greenhouse effect caused by increasing $\mathrm{CO}_{2}, \mathrm{CH}_{4}, \mathrm{~N}_{2} \mathrm{O}$, and CFCs on the current climate. Simulations with the CCM will be carried out in similar manners. In addition, the CCM will simulate energy balances at ARM testbed 
sites and in regions expected to have atmospheric radiative balances quite different from those at the ARM sites.

Information on the findings of this work that will be relevant to measurements and experiments carried out at ARM testbed sites will be provided to the ARM Science Team.

Results to Date: Total direct and diffuse solar radiation reaching the surface, longwave radiation reaching the surface, and surface meteorological data were collected for the model-observation comparison of the solar and longwave radiation. The gaseous-absorption data in our GCM longwave-radiation code were updated. The total band parameters for $\mathrm{H}_{2} \mathrm{O}, \mathrm{CO}_{2}, \mathrm{O}_{3}, \mathrm{CH}_{4}, \mathrm{~N}_{2} \mathrm{O}$, and CFCs were revised with the 1991 HITRAN absorptionline data. Extensive reviews of studies concerning the optical properties and radiative parameterizations of clouds were conducted for cirrus clouds and stratiform clouds in the effort to explicitly incorporate these optical properties into the model. For both types of clouds, the radiative effect is parameterized in terms of the singlescattering albedo, asymmetry factor, and optical thickness; for cirrus, nonspherical ice crystals are also considered.

A semiprognostic climate model consisting of the basic physical parameterizations from NCAR/GENESIS has been developed to study the resolution dependence of cloud-radiation parameterizations. By compositing the results at different horizontal scales, the horizontalresolution dependence of the cloud-radiation parameterizations can be investigated. We have tested the semiprognostic model at grid sizes from 60 to $720 \mathrm{~km}$ with an analysis of the standard radiosonde network around the SGP CART site. By maintaining consistent model physics across the full range of resolutions, the only source of differences in the final averages for the entire region is the horizontal resolution.

\section{PHOTOMETRIC STUDIES OF CLOUDS}

WHITEMAN, CHARLES D.

\section{PACIFIC NORTHWEST LABORATORY}

$\begin{array}{ll}\text { FY } 1993 & 150 \\ \text { FY } 1992 & 150 \\ \text { FY } 1991 & 150\end{array}$

Objective: To measure vertical profiles of radiative fluxes through the atmospheric boundary layer to depths of up to $1.5 \mathrm{~km}$ to supplement ground-based and aircraft-based measurements used for testing radiativetransfer models.

Product: Vertical, broadband, radiometric profiles through the atmosphere under cloudy and uncloudy conditions.

Approach: An automatic control loop will be used to level a radiometer mounting platform that will be flown in the tetherline of a small, tethered, balloon sounding system. The platform will carry sensors to measure the upward and downward-directed streams of long- and shortwave radiation. Additional sensors will measure the platform's attitude, including the azimuth and elevation angles. Data will be processed and stored on board with a small, lightweight, programmable, data-acquisition system. The balloon itself will carry a separate meteorological data-collection system to collect vertical profiles of basic meteorological data as the balloon ascends.

The project will be carried out in three phases: initial design and prototyping, further development of the prototype and completion of the data interfacing, and field testing and strengthening of the design for operational use.

Results to Date: Data from a radiometric field experiment were analyzed to test the performance of a model that we developed to calculate, on the basis of theory, the radiometric-measurement errors caused by a residual tilt of our stabilized sky platform. The model verified our original platform operating specifications.

Initial flight tests were conducted to measure the performance of the sky platform and of a motion-sensing platform, which measures tetherline motions that must be darnped by the stabilization system. The flights were conducted in a moderately sheared wintertime boundary layer and resulted in an average stabilization to within $0.8 x$ of horizontal, with oscillation standard deviations of about $2 \mathrm{x}$. This performance is suitable for producing useful vertical profiles with on-board broadband radiometers. Motion-sensing-platform data allowed us to separate angular and lateral accelerations, velocities, and displacements to characterize the tetherline motions.

$10 / 01 / 91-09 / 30 / 92$ 


\section{GLOBAL CARBON CYCLE}

Global-carbon-cycle research provides the scientific underpinnings for predicting future concentrations of $\mathrm{CO}_{2}$ in the atmosphere. The research addresses natural systems that govern the abundance of $\mathrm{CO}_{2}$ in the atmosphere, including the role of oceans and terrestrial ecosystems in determining exchanges of $\mathrm{CO}_{2}$ with the atmosphere. In addition, carbon-cycle research compiles information about technology, economics, geology, and other factors required for estimating emissions of $\mathrm{CO}_{2}$.

Major studies of the past decade have (1) measured the current and historical release of $\mathrm{CO}_{2}$ from fossil-fuel use; (2) examined the effects of existing and possible future technologies on global $\mathrm{CO}_{2}$ emissions; (3) estimated the uncertainty surrounding future global fossil-fuel $\mathrm{CO}_{2}$ emissions; (4) examined the components of the ocean carbon cycle and carbon reservoirs; and (5) described carbon dynamics for the terrestrial biosphere. Results from such studies contribute to models of the global carbon cycle, and the information is used in interim assessments of the fate of excess $\mathrm{CO}_{2}$ in global carbon reservoirs.

Major uncertainties remain about what happens to the $\mathrm{CO}_{2}$ produced by the burning of fossil fuels. The inability to balance the global carbon budget affects our ability to explain the cause of the current rate of increase, and it affects the accuracy of future atmospheric $\mathrm{CO}_{2}$ predictions.

Moreover, the uncertainties seriously limit the ability to quantify the effectiveness of possible actions that could modify the natural carbon cycle (i.e., reforestation or enhanced carbon fixation in terrestrial and marine ecosystems) and possibly slow the rise of atmospheric $\mathrm{CO}_{2}$.
Data and models on the carbon cycle are needed for national and IPCC scientific assessments of greenhouse gas issues and global climate change. Specifically, improved carbon cycle models are the tools for predicting future atmospheric $\mathrm{CO}_{2}$ concentrations, and for evaluating effectiveness of proposed greenhouse gas mitigation strategies. This includes the analysis of stabilization scenarios of the Intergovernmental Panel on Climate Change (IPCC) 1995 scientific assessment.

Research Objectives. The current program is designed to take the next step beyond the current inventory of our knowledge of the carbon cycle. It aims to increase our knowledge and understanding about the terrestrial components of the global carbon cycle to more accurately predict change of atmospheric $\mathrm{CO}_{2}$ from energy emissions. Specific objectives are (1) to understand processes controlling the exchange rate of $\mathrm{CO}_{2}$ between the atmosphere and the terrestrial biosphere, (2) to develop process-based models of carbon exchange between the atmosphere and the terrestrial environment, (3) to document energy émissions of $\mathrm{CO}_{2}$, (4) to evaluate source-sink mechanisms for atmospheric $\mathrm{CO}_{2}$, and (5) to develop reliable global carbon models for predicting future atmospheric concentrations of $\mathrm{CO}_{2}$.

Research Questions. In the carbon-cycle area:

- What additional knowledge of carbon-cycle processes is needed to accurately estimate the contribution of fossil-fuel burning to the level of $\mathrm{CO}_{2}$ in the atmosphere?

- What are the quantitative magnitudes of sources or sinks for carbon that must be considered? 
In the energy area:

-What are the scientific, technologic, economic, and geologic data needed for estimating emissions of $\mathrm{CO}_{2}$ from energy activities?

\section{Program Manager}

Roger C. Dahlman

Environmental Sciences Division

Department of Energy, ER-74

Washington, DC 20585

(301) 903-4951

Internet:

roger.dalhman@mailgw.er.doe.gov 


\section{Research Summaries}

\section{THE EFFECTS OF CHANGING LAND USE ON ORGANIC CARBON AND RADIOCARBON IN SOIL}

\author{
BROECKER, WALLACE S. \\ COLUMBIA UNIVERSITY

$\begin{array}{ll}\text { FY } 1993 & 60 \\ \text { FY } 1992 & 58 \\ \text { FY } 1991 & 60\end{array}$

$09 / 15 / 93-09 / 14 / 94$

Objective: To determine how changing land use affects organic carbon storage in soil and whether much of the missing carbon in the global carbon budget resides in soils at depths not commonly measured in basic soil surveys and to look at time histories of carbon and nitrogen accumulation under anaerobic conditions of rice paddies.

Product: Reconstructed carbon and nitrogen inventories of soil profiles in the com belt and grasslands of the central United States that have been subjected to different land uses.

Approach: Organic carbon and nitrogen fractions will be measured for cultivated soils and will be compared with samples collected at undisturbed sites not affected by past agricultural practices. Carbon lost from upper soil layers and deposited in lower soil layers instead of being oxidized to $\mathrm{CO}_{2}$ will be measured. Also, measures of bulk soil density, porosity, moisture content, N/C ratio, ${ }^{14} \mathrm{C},{ }^{137} \mathrm{Cs}$, and ${ }^{210} \mathrm{~Pb}$ in soil fractions will be used to identify processes of carbon storage in deeper soils as well as to estimate the residence time of various carbon fractions in soils.

Results to Date: Radiocarbon measurements of bulk soil from the literature and our own measurements have been used to estimate the turnover time and inventory of active soil carbon. The turnover time of passive soil carbon has been estimated to be $>1000$ years, the proportions of active (several decades) and passive soil carbon in uncultivated soil have been determined, the mean turnover time for active soil carbon has been determined, the increase in soil carbon inventories because of $\mathrm{CO}_{2}$ fertilization has been estimated, and how cultivation changed the proportions of active and passive soil carbon has been determined.

\section{SPATIAL AND TEMPORAL PATTERNS OF BIOTIC EXCHANGES OF $\mathrm{CO}_{2}$ BETWEEN THE ATMOSPHERE AND TROPICAL LANDSCAPES AND THEIR ROLE IN THE GLOBAL CARBON BALANCE}

BROWN, SANDRA

UNIVERSITY OF ILLINOIS

$\begin{array}{lr}\text { FY } 1993 & 97 \\ \text { FY } 1992 & 103 \\ \text { FY } 1991 & 102\end{array}$

$09 / 15 / 93-09 / 14 / 94$

Objective: To examine terrestrial sources and sinks for atmospheric $\mathrm{CO}_{2}$ (1) to determine historical and future spatial-temporal patterns of biotic $\mathrm{CO}_{2}$ exchange between the tropics and the atmosphere, (2) to determine the long-term (1850-1980) and short-term (1975-1990) annual net tropical carbon flux, (3) to assess the relative importance of mechanisms that control the short-term exchanges, (4) to evaluate the estimated short-term flux with measured variations in atmospheric $\mathrm{CO}_{2},(5)$ to provide a best estimate of tropical $\mathrm{CO}_{2}$ flux from 1958 to the present, and (6) to test the hypothesis that $\mathrm{CO}_{2}$ fertilization has been an important mechanism in the net tropical carbon balance. This component focuses on items (1), (2), and (3), with an emphasis on tropical Asian and American forests.

Product: A method for converting different types of structural data for forests (inventory of trees by species, diameter, and height from known size plots; stand and stock tables from large-scale forest inventories; and total commercial volumes of forests) into biomass estimates for all the tropical region; more-accurate and -precise biomass estimates for tropical American forests; models for predicting forest biomass for areas where no information is available with a geographical information system (GIS); and methods for producing spatial distributions of soil organic carbon (SOC). 
Approach: Components of the methods for estimating biomass of forests from forest inventory data have already been established under prior support. Because sufficient tropical forest inventories are lacking, new methods will be developed for predicting biomass of forests. This new method will rely on a GIS modeling approach and the assumption that the current distribution of forest biomass density is a function of the potential amount that the landscape can support under the prevailing environmental factors (precipitation, climate index, elevation, and soil texture and quality) and the cumulative impact of human activities (e.g., logging, fuelwood collection, and shifting cultivation). Simple relationships between the data layers of the environmental factors and biomass will be developed and applied to the region in a GIS to produce an index of potential biomass density of forests. The potential index will be calibrated to biomass units with data from the literature. 'To add the influence of human activities that degrade forest biomass, a series of regression equations, stratified by climate, between degradation ratios (actual biomass/potential biomass) and population density will be developed for areas where forest inventories exist. These regression equations will be used with distributions of potential biomass and population to produce a distribution of actual forest biomass. This approach will be used with distributions of contemporary and historical population densities to produce distributions of forest biomass density for several periods in time. Distributions of SOC will also be produced with a GIS approach that employs a pantropical data set of SOC densities for forest vegetation, stratified by climate and soil texture. The digital version of the Soil Map of the World, a climate map, and a forest map will then be used to assign each pixel the appropriate SOC density.

Results to Date: (1) Digital maps of potential and actual forest above- and below-ground biomass have been revised and finalized for continental and insular South and Southeast Asia. The total present (ca. 1980) above- and below-ground biomass carbon in forest vegetation for the region is $38 \mathrm{Pg}$, with a weighted mean of $132 \mathrm{Mg} / \mathrm{ha}$. At the country level, average forest biomass carbon density varies from a low of $77 \mathrm{Mg} /$ ha for India to $198 \mathrm{Mg}$ /ha for Sarawak and Sabah. (2) Digital maps of total carbon (in forest and nonforest vegetation and soils) at the subnational scale of resolution for 1880 , $1920,1950,1970$, and 1980 have also been produced as well as the differences in carbon pools (net carbon flux) among these periodș. (3) Digital maps of SOC (to 40 $\mathrm{cm}$ depth for use with modeling land-use change and to
$100 \mathrm{~cm}$ depth for estimating pools) have also been produced for the whole of tropical Asia and tropical South America. (4) The potential biomass carbon density map, forests and native nonforests, for tropical America has been completed. Progress is being made on developing forest-degradation factors to convert the potential map into an actual biomass map.

\section{ENERGY AND FUTURE CARBON DIOXIDE EMISSIONS}

EDMONDS, JAMES A.

\section{PACIFIC NORTHWEST LABORATORY}

$\begin{array}{ll}\text { FY } 1993 & 350 \\ \text { FY } 1992 & 328 \\ \text { FY } 1991 & 389\end{array}$

10/01/92-09/30/93

Objective: To develop the scientific basis to make and bound projections of future energy related emissions to the atmosphere of $\mathrm{CO}_{2}$ and other radiatively important gases and to support DOE in its participation in the Intergovernmental Panel on Climate Change (IPCC) and the Committee on Earth and Environmental Sciences (CEES).

Product: Support for DOE in its participation in the IPCC, Response Strategies Working Group, Energy and Industry Subgroup (IPCC/RSWG/EIS) and in the CEES and an energy-economic model of $\mathrm{CO}_{2}$ and other radiatively active gas emissions based on the Edmonds/Reilly model.

Approach: Quantitative support will be provided for DOE participation in the IPCC and the CEES through attendance at committee meetings.

A second-generation model will be developed with a general-equilibrium economic structure in sufficient detail to provide a framework for accounting for all significant greenhouse gases. As a point of departure, a Version 0.0 will be developed first to demonstrate not only the concept but also several computational techniques. Energy activities will be the principal focus of the mode. Other economic interactions will be highly aggregated. The initial version will model 11 sectors (agriculture, crude oil, refined oil, natural gas, coal, biomass, uranium, electricity, other products, households, and government) and 12 factors of 
production (agriculture, crude oil, refined oil, natural gas, coal, biomass, uranium, electricity, other products, land, labor, and capital). Capital stocks will be tracked by year of installation. International trade will be allowed. A demographic module of population growth will be developed that will facilitate the analysis of interactions among population growth, labor force, economic growth, energy use, and greenhouse-gas emissions. This prototype will provide the basis for evolutionary development of the full scale version of the model.

Results to Date: Data for the United States was applied to the second-generation model (SGM) Version 0.0 . The model has been applied to the analysis of potential emissions reductions strategies for the United States. Results show the importance of the internal demographic module and the ability to specify explicitly the disposition of revenues from fiscal strategies. A collaboration between PNL. and the Indian Institute of Management has lead to the near completion of an Indian data set for the SGM. A data set for China is substantially complete, but problems with immigration regulations led to an unanticipated delay. Work on the Western European module was initiated with the Centre International de Recherche sur l'Environnement et le Développement, and a data set is expected to be completed in 1994. Work has also begun on the Japanese and South Korean modules through collaborations with the Japanese Ministry of the Environment and the Korean Energy Economics Institute.

\section{GLOBAL CARBON CYCLE STUDIES}

\author{
EMANUEL, W. R.; KING, A. W.; PENG, T.-H.; and \\ POST, W. M.
}

\section{OAK RIDGE NATIONAL LABORATORY}

$\begin{array}{ll}\text { FY } 1993 & 1200 \\ \text { FY } 1992 & 1300 \\ \text { FY } 1991 & 1265\end{array}$

$10 / 01 / 92-09 / 30 / 93$

Task 1. Terrestrial Carbon Cycle Model Development

Objective: To develop a mathematical nuodel of carbon cycling in the world's terrestrial ecosystems that can simulate responses to land use, climatic change, and rising atmospheric $\mathrm{CO}_{2}$ concentration on time scales from decades to several centuries.

Product: A model of the terrestrial component of the global carbon cycle with data sets, standard test cases, and documentation.

Approach: Two classes of models must be merged to realistically describe terrestrial carbon dynamics. This requirement arises because, although the slow circuit of carbon through vegetation and dead organic matter is responsible for significant carbon storage, the influence of environmental conditions occurs within much-fasterresponding carbon circuits: photosynthesis, respiration, translocation, and transpiration in plants and the turnover of microbial decomposers in actively decomposing organic-matter pools. Thus two modules will be formulated with two sets of characteristic response times to describe terrestrial carbon dynamics: (1) A rapid module will describe $\mathrm{CO}_{2}$ exchanges, photosynthesis, water loss, and energy balance for a prototype leaf or photosynthetic unit. (2) A slow dynamics module will represent ecosystem carbon storage as a compartmental system of living and dead components, the fluxes between them, and atmospheric exchanges.

Human activities affect a large fraction of the world's terrestrial ecosystems. These disturbances (which range from total management, harvest, and land-use change to subtle pollutant impacts) cannot be ignored in any analysis of changes in atmospheric $\mathrm{CO}_{2}$ over periods longer than a few years.

Our terrestrial carbon-cycling model will divide the Earth's surface into land units of arbitrary geography depending on an application's needs for spatial detail and the organization of supporting data. Each land unit will be further subdivided into ecosystems, but the model will track only their extents, not their locations, within land units. Carbon exchanges between the atmosphere and all ecosystems within a land unit will be assumed to occur at the land unit's centroid. An area distribution function will further describe variations of carbon density in vegetation per unit area for each ecosystem within each land unit. Land-use activities and plant growth will alter these area-distribution functions through time, generally with opposing tendencies.

Results to Date: A terrestrial carbon cycle model that simulates responses to land use, climate change, and atmospheric $\mathrm{CO}_{2}$ concentration was completed and 
applied in sensitivity studies of the effects of rising $\mathrm{CO}_{2}$ on plant growth. A selection of growth-response functions were calibrated to experimental results, and the terrestrial carbon-cycle model was used to simulate the net flux from plants and soil into the atmosphere that was caused by past land use. The net flux was significantly smaller in all cases in which plant response to $\mathrm{CO}_{2}$ was assumed, but the plant response did not account for all of the difference between model solutions and observations. The plant response was limitad by assumptions about carrying capacity for carbon storage in terrestrial ecosystems, and these studies show that a model that explicitly treats processes is needed to fully evaluate the influence of atmospheric $\mathrm{CO}_{2}$ on terrestrial carbon cycling. A photosynthesis model and an extended model of soil carbon turnover are being incorporated into the tetrestrial carbon cycle model to satisfy this requirement.

Task 2. Total-Carbon-Cycle Models and Changes in Atmospheric $\mathrm{CO}_{2}$.

Objective: To clarify the relationships between fossilfuel emissions and atmospheric $\mathrm{CO}_{2}$ concentration that control further $\mathrm{CO}_{2}$ increases as fossil-fuel use continues.

Product: Models of the global carbon cycle that include representations of carbon dynamics in the terrestrial and oceanic reservoirs with underlying data and analyses to clarify potential responses of atmospheric $\mathrm{CO}_{2}$ to further fossil-fuel use.

Approach: Purely' observational studies of the phenomena controlling $\mathrm{CO}_{2}$ concentration are impractical because of the time scales involved (decades to centuries), the vast extent and spatial heterogeneity of Earth systems, and the small perturbations to natural levels that fossil-fuel releases cause in very large reservoirs, such as the oceans. Furthermore, many mechanistically important variables cannot be measured directly. Mathematical models are an important means of contending with these limitations of direct studies. They also provide a means of synthesizing the diverse data assembled to address the $\mathrm{CO}_{2}$ issue and of interpreting these data in terms of changes in atmospheric $\mathrm{CO}_{2}$ levels.

To estimate changes in atmospheric $\mathrm{CO}_{2}$ concentration that are caused by different amounts of fossil-fuel use, the influences of the oceans, land plants, and dead organic matter and its decomposers in soil must be considered. These parts of the global carbon cycle certainly affect $\mathrm{CO}_{2}$ levels over decades and centuries. A total-carbon-cycle approach will be taken; and data, concepts, and models from atmospheric, terrestrial, and oceanic projects will be integrated.

Estimates of fossil-fuel $\mathrm{CO}_{2}$ emissions will be derived largely from data compiled by the United Nations Statistics Office. These data do not include details of energy end-uses that allow analysis of the relationships between $\mathrm{CO}_{2}$ emissions and socioeconomic factors. With energy data increasingly available with increasing accuracy and detail, we will reexamine the accuracy of the data and the differences between data sets. We will also analyze the energy end-uses most related to $\mathrm{CO}_{2}$ emissions to describe where $\mathrm{CO}_{2}$ emissions are concentrated within the global economy.

Results to Date: Coupled to a model of carbon turnover in the atmosphere and oceans, the extended model of terrestrial carbon cycling developed by this project permits analysis of land-use perturbations and climatic change on atmospheric $\mathrm{CO}_{2}$ concentration. The response of terrestrial ecosystems also affects estimates of the lifetime of $\mathrm{CO}_{2}$ in the atmosphere as it is used in global-warming-potential indices. The solution of this total-carbon-cycle model cannot be made to conform to observations under current most-likely assumptions about past fossil-fuel emissions, land use, and best estimates of parameter values. Some of this discrepancy is resolved by including the dependence of plant growth on atmospheric $\mathrm{CO}_{2}$ concentration, but the disagreement with observations is still large enough to make it difficult to project future $\mathrm{CO}_{2}$ levels with the model. The character of the differences between model solutions and observations can be analyzed by calculating the net flux into or out of the atmosphere required to resolve the discrepancy. Interannual variations in this residual net flux appear to be associated with El Niño/Southern Oscillation events and other environmental fluctuations, and the role of terrestrial ecosystems in controlling changes in atmospheric $\mathrm{CO}_{2}$ will be further clarified by analyzing these shorter-term variations. 


\section{SPATIAL AND TEMPORAL PATTERNS OF BIOTIC EXCHANGES OF $\mathrm{CO}_{2}$ BETWEEN THE ATMOSPHERE AND TROPICAL LANDSCAPES AND THEIR ROLE IN THE GLOBAL CARBON BALANCE}

FUNG, I. Y.-S

\section{GODDARD INSTITUTE FOR SPACE STUDIES}

$\begin{array}{ll}\text { FY 1993 } & 110 \\ \text { FY 1992 } & 109 \\ \text { FY 1991 } & 104\end{array}$

09/15/93-09/14/94

Objective: To reduce the uncertainty related to estimates of the net flux of carbon between terrestrial ecosystems and the atmosphere because of deforestation and natural feedbacks in the tropics.

Product: A justified reanalysis of the net flux of carbon to the atmosphere as a result of anthropogenic changes and natural atmospheric/biospheric feedbacks, especially in the tropics. The reanalysis will involve the use of all available flux data and will be constrained by the observed concentrations and gradients of atmospheric $\mathrm{CO}_{2}$ and its isotopes.

Approach: The atmospheric, oceanic, and terrestrial reservoirs have different isotopic signatures. Also, fractionation effects on the different exchange processes and distributions of the isotopic concentration of $\mathrm{CO}_{2}$ in all three reservoirs provide additional information about $\mathrm{CO}_{2}$ exchanges.

The GISS 3-D atmospheric-transport model will be used to simulate atmospheric $\mathrm{CO}_{2}$ and ${ }^{13} \mathrm{CO}_{2}$ responses to refined estimates of carbon fluxes. The modeled concentrations and gradients will be compared with those observed.

Results to Date: Our research continues to address uncertainties of the global carbon budget. Analyses with the atmospheric $\mathrm{CO}_{2}$ circulation model provide estimates of regionally differentiated $\mathrm{CO}_{2}$ sources and sinks with special attention to $\mathrm{CO}_{2}$ exchanges between the atmosphere and biosphere.

The 3-D atmospheric $\mathrm{CO}_{2}$ transport model that is sectorially linked to oceans and landscapes to evaluate $\mathrm{CO}_{2}$ exchanges is being used to calculate $\mathrm{CO}_{2}$ fluxes between biosphere and atmosphere from from inferred fluxes based on terrestrial biomass data and land-use change. Fluxes between atmosphere and ocean are being estimated from $\mathrm{pCO}_{2}$ differences and ocean-surface properties. These calculated, integrated, net $\mathrm{CO}_{2}$ fluxes are then being calibrated against atmospheric $\mathrm{CO}_{2}$ monitoring data from a network of worldwide stations and with $8^{13} \mathrm{C}$ signatures in the atmosphere.

\section{SPATIAL AND TEMPORAL PATTERNS OF BIOTIC EXCHANGES OF $\mathrm{CO}_{2}$ BETWEEN THE ATMOSPHERE AND TROPICAL LANDSCAPES AND THEIR ROLE IN THE GLOBAL CARBON BALANCE}

HALL, CHARLES A. S.

\section{STATE UNIVERSITY OF NEW YORY. AT SYRACUSE}

$$
\begin{array}{lr}
\text { FY } 1993 & 150 \\
\text { FY } 1992 & 149 \\
\text { FY } 1991 & 146 \\
& \\
\multicolumn{2}{c}{09 / 15 / 93-0 \% / 14 / 94}
\end{array}
$$

Objective: To finish refining the methods we use to predict large-scale spatial patterns of land-use change, to convert models of global carbon flux and land-use change into a GIS format, and to apply these models to Southeast Asia and Central America.

Product: Much more spatially refined estimates of carbon release as a consequence of land-use change.

Approach: Historical patterns of land-use change will be analyzed to validate our land-use-change predictor. Our spatial work based on historical maps and digital map making will be augmented with satellite imagery. Digital maps of elevation, vegetation, and land use will be prepared; and the application and sensitivity analysis of a computer model (GEMOD) that predicts the spatial pattern and temporal dynamics of land-use types for a given region will be made interactive with digitized maps or images.

Results to Date: Our initial approach to model land-use change was to use elevation and slope as pattern drivers and long-term population increase as a rate driver. Our model started with "seeds" of initial development that then spread development to neighboring, low-lying, flat areas. With this approach, we recreated basic land-use 
rates and patterns for montane regions of Asia. But this approach failed for Africa, so we generalized our model to use a custom-designed statistical technique to identify empirically the best pattern drivers from a set of maps including topography, soil, precipitation, and other factors. We used the inproved empirical approach to simulate land-cover change in the Zaire Basin from 1880 to 2080 , and are now ready to apply GEOMOD to the whole tropics.

\section{REMOTE SENSING OF MESOSCALE VARIABILITY}

\section{HOSKER, R. P. \\ ATMOSPHERIC TURBULENCE AND DIFFUSION DIVISION, NOAA}

$\begin{array}{lr}\text { FY } 1993 & 120 \\ \text { FY } 1992 & 0 \\ \text { FY } 1991 & 0\end{array}$

07/01/93-09/30/93

Objective: To participate in coordinated field measurements of $\mathrm{CO}_{2}$ flux in the Alaskan arctic.

Product: A data set of net $\mathrm{CO}_{2}$ flux across different vegetated surfaces of the Alaskan tundra for comparison with other real-time measurements of $\mathrm{CO}_{2}$ flux to determine if tundra ecosystems are taking up carbon (functioning as sinks) or releasing carbon to the atmosphere as a biotic source.

Approach: The NOAA Atmospheric Turbulence and Diffusion Group at Oak Ridge will use eddy-covariance techniques and an ultralight aircraft to measure $\mathrm{CO}_{2}$ fluxes over large areas simultaneously with groundbased techniques using pop-on chambers and stationary tower sensors. A "mini" field campaign will be conducted in late August of 1993 in collaboration with Canadian scientists taking similar measurements by aircraft and simultaneously with Japanese scientists making stationary tower measurements and other U.S. scientists measuring $\mathrm{CO}_{2}$ fluxes of various stands of vegetation. The independent measurements will be compared for consistency of direction and magnitude of $\mathrm{CO}_{2}$ flux.

Results to Date: New project.

\section{SPATIAL AND TEMPORAL PATTERNS OF BIOTIC EXCHANGES OF $\mathrm{CO}_{2}$ BETWEEN THE ATMOSPHERE AND TROPICAL LANDSCAPES AND THEIR ROLE IN THE GLOBAL CARBON BALANCE}

\author{
HOUGHTON, R. A.
}

WOODS HOLE RESEARCH CENTER

$\begin{array}{ll}\text { FY } 1993 & 239 \\ \text { FY } 1992 & 225 \\ \text { FY } 1991 & 212\end{array}$

$09 / 15 / 93-09 / 14 / 94$

Objective: To examine terrestrial sources and sinks for atmospheric $\mathrm{CO}_{2}$ (1) to determine historical and future spatial-temporal patterns of biotic $\mathrm{CO}_{2}$ exchange between the tropics and the atmosphere, (2) to determine the long-term (1850-1980) and short-term (1975-1990) annual net tropical carbon flux, (3) to assess the relative importance of mechanisms that control the short-term exchanges, (4) to evaluate the estimated short-term flux with measured variations in atmospheric $\mathrm{CO}_{2}$, (5) to provide a best estimate of tropical $\mathrm{CO}_{2}$ flux from 1958 to the present, and (6) to test the hypothesis that $\mathrm{CO}_{2}$ fertilization has been an important mechanism in the net tropical carbon balance. This component focuses on Item (3).

Product: A refined range of estimates of the net flux of carbon to the atmosphere from tropical deforestation and reforestation for the 1980s and new estimates of the integrated flux between 1850 and 1990.

Approach: Existing statistics on carbon content of vegetation and soils will be assembled, and a simple carbon-balance model will be used to estimate $\mathrm{CO}_{2}$ flux. The analysis will concentrate on changes involving tropical forests; however, the estimates will be combined with data from other geographic areas to provide a global estimate of the $\mathrm{CO}_{2}$ flux from land clearing and other human influences. As new data are reported in the open literature, they will be promptly incorporated into the global $\mathrm{CO}_{2}$ analysis.

Results to Date: The approach we have used to calculate worldwide emissions of carbon from terrestrial ecosystems is based on changes in land use (rates of deforestation and reforestation) and changes in vegetation and soils in response to land-use change. 
Geophysicists have used a different approach (deconvolution), based on models of oceanic uptake and past atmospheric concentrations of $\mathrm{CO}_{2}$, to calculate the terrestrial flux of carbon. The two approaches show different patterns of carbon release over the period 1850 to 1990: the flux of carbon from changes in land use has increased over time; the flux determined from deconvolution has decreased since about 1920. However, the two approaches address different components of a terrestrial flux of carbon. The flux based on deconvolution is a net flux that includes the land-use flux as well as other biotic fluxes. Assuming that both estimates are correct, the difference between them defines the "missing carbon sink" during the past 140 years. For most of this period, carbon appears to have been accumulating on land at a rate proportional to industrial activity (suggestive of $\mathrm{CO}_{2}$ fertilization or increased nitrogen deposition). Since 1975, the rate of this accumulation seems to have slowed (suggestive of a warming-enhanced rate of respiration).

\section{A STUDY OF THE ABUNDANCE AND ${ }^{13} \mathrm{C} /{ }^{12} \mathrm{C}$ RATIO OF ATMOSPHERIC CARBON DIOXIDE AND OCEANIC CARBON IN RELATION TO THE GLOBAL CARBON CYCLE}

\author{
KEELING, CHARLES D.
}

\section{SCRIPPS INSTITUTION OF OCEANOGRAPHY}

$\begin{array}{ll}\text { FY } 1993 & 285 \\ \text { FY } 1992 & 275 \\ \text { FY } 1991 & 268\end{array}$

07/01/93-06/30/94

Objective: To continue high-precision atmospheric $\mathrm{CO}_{2}$ measurements at the long-term monitoring sites (e.g., Mauna Loa Observatory, the South Pole, and other stations in the Pacific Ocean Basin); to continue the measurement of isotopic carbon in the atmospheric $\mathrm{CO}_{2}$ samples collected from the long-term $\mathrm{CO}_{2}$ monitoring stations; to expand measurements of both $\mathrm{CO}_{2}$ and isotopic composition at several North American land stations; and to use the high-precision data in models of the global carbon cycle.

Product: A database for calibrating atmospheric $\mathrm{CO}_{2}$ circulation models and for evaluating sources and sinks of exchange with oceans and the terrestrial biosphere.
Approach: High-precision $\mathrm{CO}_{2}$ and isotopic measurements will be carried out at background air pollution monitoring stations of the Central Pacific region (e.g., the Mauna Loa, Hawaii location). Atmospheric $\mathrm{CO}_{2}$ data will be collected at additional sites to create a database for validating 3-D models of atmospheric and oceanic transport of carbon in the atmosphere and oceans. The expanded data base, including isotopic data frorn additional Northern Hemisphere locations, will be used to test the hypothesis that the warming trend of the 1980 s may in part be affecting the atmospheric $\mathrm{CO}_{2}$ concentration and the natural carbon cycle. Analysis with the combined database and model will help to both detect and understand causes of $\mathrm{CO}_{2}$ signals related to large-scale phenomena, such as El Niño of the equatorial Pacific, and temperature/fertilization effects on terrestrial biota. The improved-resolution models will be used to predict atmospheric $\mathrm{CO}_{2}$ change in relation to changing processes and properties of the global carbon cycle.

Results to Date: According to data obtained from the Arctic to the South Pole, the concentration of atmospheric $\mathrm{CO}_{2}$ has continued to increase during the past 18 months but at a lower rate than expected from the combustion of fossil fuels. The lower rate is not accompanied by a large anomaly in the ${ }^{13} \mathrm{C} /{ }^{12} \mathrm{C}$ ratio of $\mathrm{CO}_{2}$. The decreased concentration rise is evidently caused by an anomalous oceanic absorption, which is in addition to absorption related to the buildup of atmospheric $\mathrm{CO}_{2}$ from fossil-fuel combustion. The anomalous absorption, about 4 billion metric tons of carbon, caused the total absorption to be double the long-term average and thus equal to about $85 \%$ of the $\mathrm{CO}_{2}$ emitted to the air. Model calculations indicate that only a small part of the absorption is likely to be caused by cooling of surface water. The main cause, therefore, must be an increase in photosynthetic fixation of carbon in the oceans. The uptake is similar in magnitude to that after the 1982 El Niño event, except that then the oceanic uptake was accompanied by increased release of $\mathrm{CO}_{2}$ by land vegetation. During the past 18 months, the land vegetation seems not to have perturbed atmospheric $\mathrm{CO}_{2}$, leaving only the strong perturbation by marine life in the oceans. 


\section{SPATIAL AND TEMPORAL PATTERNS OF BIOTIC EXCHANGES OF $\mathrm{CO}_{2}$ BETWEEN THE ATMOSPHERE AND TROPICAL LANDSCAPES AND THEIR ROLE IN THE GLOBAL CARBON BALANCE}

RICHARDS, JOHN F.

DUKE UNIVERSITY

$\begin{array}{lr}\text { FY } 1993 & 96 \\ \text { FY 1992 } & 99 \\ \text { FY 1991 } & 100\end{array}$

$09 / 15 / 93-09 / 14 / 94$

Objective: To examine terrestrial sources and sinks for atmospheric $\mathrm{CO}_{2}$ (1) to determine historical and future spatial-temporal patterns of biotic $\mathrm{CO}_{2}$ exchange between the tropics and the atmosphere, (2) to determine the long-term (1850-1980) and short-term (1975-1990) annual net tropical carbon flux, (3) to assess the relative importance of mechanisms that control the short-term exchanges, (4) to evaluate the estimated short-term flux with measured variations in atmospheric $\mathrm{CO}_{2}$, (5) to provide a best estimate of tropical $\mathrm{CO}_{2}$ flux from 1958 to the present, and (6) to test the hypothesis that $\mathrm{CO}_{2}$ fertilization has been an important mechanism in the net tropical carbon balance. This component focuses on items (1) and (2).

Product: Time-series data on carbon balance for land components (vegetation and soil) in relation to agriculture and increased population in Southeast Asia and Central and South America.

Approach: A historical record of land use will be constructed to approximate the preindustrial $\mathrm{CO}_{2}$ flux from human influences. These reference data, when combined with fossil-fuel $\mathrm{CO}_{2}$ emissions, will be valuable for modeling the time history of the global carbon cycle. There are no alternative approaches for reconstructing this type of information. Area figures for land use at each level will be converted into estimates of carbon in standing stock of vegetation. These estimates will be based upon a large, regional, ecological and botanical literature measuring biomass for various types of vegetation.

Results to Date: The database of land use and carbon content of live vegetation was completed and will be distributed in electronic form by the Carbon Dioxide
Information Center at Oak Ridge National Laboratory. In addition to the tabular data, back-up data, calculations, documentation and bibliography are attached in the form of more than 2000 spreadsheets available on demand. This database synthesizes intensive historical and ecological research carried out during the past 10 years.

\section{SIMULATIONS OF THE CARBON CYCLE IN THE OCEANS (PART I)}

$$
\text { SARMIENTO, JORGE L. }
$$

\section{PRINCETON UNIVERSITY}

$\begin{array}{lll}\text { FY } 1993 & 345 \\ \text { FY } 1992 & 288 \\ \text { FY } 1991 & 286\end{array}$

$09 / 15 / 93-09 / 14 / 94$

Objective: To develop 3-D models of the ocean carbon cycle, to perform a fixed atmospheric $\mathrm{CO}_{2}$ simulation with nonseasonal and seasonal ocean circulation, to predict background total carbon fields, to use a seasonal 3-D North Atlantic ecosystem model to do carbon and nitrogen simulations, to perform simulations with a global carbon cycle ecosystem model, and to estimate anthropogenic $\mathrm{CO}_{2}$ uptake by oceans.

Product: 3-D ocean-model simulations of the uptake of anthropogenic $\mathrm{CO}_{2}$, including possible feedback effects from changes in the ocean carbon cycle.

Approach: Two parallel approaches will be taken. The first assumes that the preanthropogenic ocean carbon cycle continues to operate without being affected directly by anthropogenic perturbations. This steadystate approach enables us to treat the anthropogenic $\mathrm{CO}_{2}$ as a passive tracer. The only information we need regarding the carbon cycle, other than the appropriate thermodynamic constants, is the preanthropogenic surface total carbon and alkalinity concentrations. The second approach assumes that the oceanic carbon cycle will quite likely respond dynamically to the effect of the anthropogenic-greenhouse-gas perturbations on climate. The ecosystem models needed to implement this approach will be placed into our ocean-circulation models.

Results to Date: Ocean-GCM simulations of the contribution of the solubility and biological pumps to 
the air-sea $\mathrm{CO}_{2}$ flux have almost been completed. These models are being used to simulate anthropogenic $\mathrm{CO}_{2}$ uptake and to suggest where improved measurements of $\mathrm{CO}_{2}$ fluxes would help to best constrain the oceanic sink. The ocean-GCM results are being combined with the results from our new atmospheric-GCM simulation of $\mathrm{CO}_{2}$ transport divergence to estimate the spatial distribution of the missing terrestrial sink. The temporal history of the missing sink has also been estimated with the GCM model results, and a simple model of the terrestrial biosphere has been developed to assess the potential role of $\mathrm{CO}_{2}$ fertilization in explaining this sink. Simulations of ${ }^{13} \mathrm{C}$ carried out in this project will be used to further evaluate the nature of the missing sink.

A new global ecosystem carbon and nitrogen simulation is being carried out to evaluate the role of seasonality in the air-sea flux of $\mathrm{CO}_{2}$. This model, modified to take into consideration the results from our new multiplesize-class ecosystem model, will be used in the future to predict the response of the ocean carbon cycle to greenhouse warming and changes in ocean circulation.

\section{SIMULATIONS OF THE CARBON CYCLE IN THE OCEANS (PART II)}

$$
\text { SIEGENTHALER, U. }
$$

\section{UNIVERSITY OF BERN}

$\begin{array}{ll}\text { FY } 1993 & 58 \\ \text { FY } 1992 & 77 \\ \text { FY } 1991 & 50\end{array}$

$$
\text { 09/15/93-09/14/94 }
$$

Objective: To develop 3-D models of the oceanic carbon cycle for simulating the ocean's uptake of anthropogenic $\mathrm{CO}_{2}$.

Product: Three-dimensional-model simulations of anthropogenic $\mathrm{CO}_{2}$ uptake with indications of the sensitivity of that uptake to various factors; simulations with a 1.5-D model; and comparisons of 3-D model results with 1.5-D model results to better understand simple models as economical substitutes for 3-D models.

Approach: The 3-D model runs will be carried out at Princeton University. The University of Bern will help to plan and evaluate the model experiments, based on our experience with simple carbon-cycle models. Simple models that allow extensive sensitivity tests and comparison of different oceanic tracer fields will be developed and used. Circulation and tracer fields from the 3-D model will be projected to 1 and 2 dimensions for deriving parameterized transport coefficients comparable to those used in simple models.

Results to Date: The 1.5-D advective-diffusive ocean tracer model HILDA has been applied further. A dynamic land-biota submodel, consisting of four boxes, has been set up. It includes regrowth of deforested areas and allows study of the response of vegetation and soil biomass to deforestation and $\mathrm{CO}_{2}$ fertilization. The completed carbon-cycle model has been run with the 1944 IPCC scenarios, and it will allow one to study the role of the land biota in more detail.

Apparent eddy diffusivities have been calculated from the output of the Princeton 3-D ocean GCM by representing the spatially averaged $\mathrm{CO}_{2}$ and ${ }^{14} \mathrm{C}$ transport fluxes by eddy diffusion and vertical advection. The results show qualitatively the same pattern as the HILDA transport parameters, confirming that eddy diffusion is a reasonable parameterization of large-scale oceanic transport for these tracers.

\section{SPATIAL AND TEMPORAL PATTERNS OF BIOTIC EXCHANGES UF $\mathrm{CO}_{2}$ BETWEEN THE ATMOSPHERE AND TROPICAL LANDSCAPES AND THEIR ROLE IN THE GLOBAL CARBON BALANCE}

\author{
SKOLE, D. L., and MOORE, B., III \\ UNIVERSITY OF NEW HAMPSHIRE
}

$\begin{array}{ll}\text { FY 1993 } & 110 \\ \text { FY 1992 } & 105 \\ \text { FY 1991 } & 100\end{array}$

$09 / 15 / 93-09 / 14 / 94$

Objective: To examine terrestrial sources and sinks for atmospheric $\mathrm{CO}_{2}$ (1) to determine historical and future spatial-temporal patterns of biotic $\mathrm{CO}_{2}$ exchange between the tropics and the atmosphere, (2) to determine the long-term (1850-1980) and short-term (1975-1990) annual net tropical carbon flux, (3) to assess the relative importance of mechanisms that control the short-term exchanges, (4) to evaluate the estimated short-term flux with measured variations in atmospheric $\mathrm{CO}_{2},(5)$ to provide a best estimate of tropical $\mathrm{CO}_{2}$ flux from 1958 to the present, and (6) to test the hypothesis that $\mathrm{CO}_{2}$ 
fertilization has been an important mechanism in the net tropical carbon balance. This component focuses on items (1) and (4).

Product: Maps and spatial integration of data on historical deforestation from tabular sources, encoding of satellite derived maps of deforestation, spatially referenced above- and below-ground carbon maps, and 2-D estimates of the net flux of carbon from tropical deforestation.

Approach: The vast amount of observations from space are used in conjunction with traditional sources of landuse data to develop a comprehensive analysis of landcover conversion. GIS technology will be used to manage and integrate land-cover-conversion data with biomass and soil organic matter in geographically referenced carbon models.
Results to Date: Geographically referenced deforestation data sets have been developed by comparing tabular estimates and remote sensing. The effort has focused on the Amazon Basin of Brazil, where approximately 30\% of the world's deforestation and $\mathrm{CO}_{2}$ emissions are. By combining tabular data from standard agriculture and land-use statistical series with maps derived from satellite remote sensing, a long-term (1960-1990) time series of deforestation rates can be developed. This work suggests that previous estimates of deforestation may have been too high, that a large amount of secondary succession exists in tropical forests, and that the net flux needs to be re-evaluated. In addition, remote-sensing analyses of deforested areas in a test site in Thailand have been compared with land-use model simulations. These results suggest rule-based models may be more accurate than regressions based on such variables as population density. 


\section{VEGETATION RESEARCH}

This Program provides information about (1) the effects of $\mathrm{CO}_{2}$ and climate on plants and the vegetative component of ecosystems and (2) plant physiological controls that affect exchange of $\mathrm{CO}_{2}$ between the atmosphere and terrestrial biota. The research strategy is to determine primary effects of increasing $\mathrm{CO}_{2}$ and temperature or moisture stress for representative plants, and to develop modeling approaches for extrapolating basic information on processes and controls to other plant and ecological systems. A key facet of the research is to acquire new experimental data and to develop and validate models for understanding the effects of altered $\mathrm{CO}_{2}$ and climate conditions on plants and ecosystems. Once developed and tested, the models would be applied to different types of vegetation (e.g., forests and rangelands) and at larger geographic scales.

The research also produces fundamental data for evaluating the potential of $\mathrm{CO}_{2}$ sequestration and quantitative data on sinks for carbon in terrestrial ecosystems. This information is important for nearterm policy analysis of $\mathrm{CO}_{2}$ mitigation by natural ecosystems and for the estimation of greenhousegas offsets under consideration by the U.S. Government Climate Change Mitigation Strategy. Information on terrestrial $\mathrm{CO}_{2}$ sequestration is also required for analyzing $\mathrm{CO}_{2}$-stabilization scenarios of the science assessment of the IPCC.

Studies of plants involve physiology, biochemistry, and whole-plant responses with emphasis on fundamental mechanisms. At the larger scale, the research focuses on productivity and structural and functional properties of ecosystems and on plantanimal-microbial relationships. Fundamental plant processes (e.g., carbon metabolism and water balance) and ecosystem processes (e.g., productivity and nutrient turnover) are examined as they might be affected directly by $\mathrm{CO}_{2}$ and indirectly by changing climate conditions. Primary products of the research are experimental data and process models of plant response to variable $\mathrm{CO}_{2}$ and climate conditions.

Research Objectives. The objectives are:

- Understanding plant physiological mechanisms that control $\mathrm{CO}_{2}$ fixation and terrestrial ecosystems' net $\mathrm{CO}_{2}$ uptake by plants; this involves experiments to determine the role of plants and terrestrial ecosystems as sinks in the global carhon balance.

- Experimentally determining the relative effects of increased $\mathrm{CO}_{2}$ and altered climate conditions on representative plants and ecosystem.

- Developing models for predicting the effects of changing $\mathrm{CO}_{2}$ and climate on vegetation.

Research Questions. Examples of questions to be addressed are:

- How much do plant growth, productivity, and carbon content change in relation to increasing atmospheric $\mathrm{CO}_{2}$ and associated climate changes? What are the interactive effects of simultaneous changes of these and other variables on photosynthesis and transpiration?

- How will functional and structural properties of plants and ecosystems change with different $\mathrm{CO}_{2}$ and climate regimes?

- What models or approaches are needed to evaluate the combined responses of plants to $\mathrm{CO}_{2}$ and other climate variables and to translate information on biological and ecological processes into estimates of larger-scale responses to global change? 
- What are the general mechanisms controlling $\mathrm{CO}_{2}$ fixation of terrestrial biota, and how do the controls operate to sequester carbon of major vegetation types?

- How can plant carbon sequestration be best modeled to determine the role of terrestrial vegetation in balancing the global carbon budget?

\section{Program Manager}

Roger C. Dahlman

Environmental Sciences Division

Department of Energy, ER-74

Washington, DC 20585

(301) 903-4951

Internet:

roger.dalhman@mailgw.er.doe.gov 


\section{Research Summaries}

\author{
SIMULATION MODELING OF CROP \\ RESPONSE TO $\mathrm{CO}_{2}$ ENRICHMENT \\ ACOCK, BASIL \\ SYSTEMS RESEARCH LABORATORY \\ $\begin{array}{lr}\text { FY } 1993 & 40 \\ \text { FY } 1992 & 0 \\ \text { FY } 1991 & 0\end{array}$
}

09/01/93-08/31/94

Objective: To define a generic modular structure for models of plant growth and then develop and test modules needed as components of modular crop models; to perform sensitivity/uncertainty analysis of the soybean crop model GLYCIM; and to exercise the models in studies of the combined effects of $\mathrm{CO}_{2}$ and temperature on plant growth.

Product: A validated, mechanistically based plantgrowth-simulation model that can treat $\mathrm{CO}_{2}$-dependent physiological processes of the whole plant and the associated environmental factors affecting growth.

Approach: The generic approach will incorporate essential $\mathrm{CO}_{2}$-dependent processes and interactions as well as physiology and growth processes that are common to many plant species. Modular concepts will be employed, and ways will be explored to aggregate plant processes to represent generic functions by analytical approaches described for large-area forecasts.

The 2-D soil model SOILSIM will treat movement of water and solutes in both the unsaturated (rooted) and saturated portions of the soil. SOILSIM will be incorporated into GLYCIM and will be validated with data sets collected from all over the United States.

Sensitivity/uncertainty analysis of the soybean GLYCIM model will continue. Because this model is the most thoroughly tested of all physiologically based plant models, aggregate predictions will attempt to account for production over wide areas. The models will attempt to link to the lower boundary of the GCMs, with initial attention to partitioning of energy at the surface. Albedo and stomatal conductance will be the initial processes examined.
Refinement of plant-model controls on carbon fixation and use in growth will be completed for $\mathrm{COTCO} 2$, a state-of-the-art plant-growth model developed jointly by DOE and U.S. Department of Agriculture Agricultural Research Service (USDA/ARS). Data from other $\mathrm{CO}_{2}$ temperature experiments with wheat will be adapted to existing wheat models, and a new model will be developed for rice.

Results to Date: A cotton-growth and -phenology model was developed and integrated into a comprehensive cotton simulation model (Sigma +), which is written in an object-oriented programming language $\left(\mathrm{C}^{++}\right)$. More than 50 data sets were collected for validation.

Weather and soil data sets were gathered for lowa in a study of methods for using field-scale crop models to make predictions about large areas. Methods were developed for describing the variability of soils within soil series. GLYCIM was validated for several soil and weather scenarios in Iowa.

Flowering in soybean is not dependent on photoperiod up to anthesis but only during specific phases. The lengths of the photoperiod-sensitive and -insensitive phases are functions of photoperiod during the sensitive phase.

Several methods of validation and parameterization of photosynthesis models were examined, and an algorithm for the photosynthetic models' evaluation was formulated. The hyperbolic model was found to be adequate and can be used in comprehensive crop-simulation models.

A 2-D soil model, 2DSOIL, was developed in modular structure and interfaced with a simple plant model. A detailed heat-balance component has been added to allow soil temperature to respond to solar radiation and air temperature. In addition, an improved method to interpolate soil hydraulic properties from measured data has been added. 


\section{ASSESSMENT OF CROP RESPONSE TO INCREASED ATMOSPHERIC CARBON DIOXIDE: RISING $\mathrm{CO}_{2}$ EFFECTS ON RICE}

ALLEN, L. H.

PLANT STRESS AND PROTECTION RESEARCH UNIT

$\begin{array}{lr}\text { FY } 1993 & 125 \\ \text { FY } 1992 & 0 \\ \text { FY } 1991 & 0\end{array}$

09/01/93-08/31/94

Objective: To determine the direct effects of $\mathrm{CO}_{2}$ and the interactive effects of temperature, water management, and nutrition on rice photosynthesis, growth, water use, and yield and to develop and/or adapt process-level crop model(s) for prediction of rice's growth and yield responses to elevated $\mathrm{CO}_{2}$ and to environmental and germplasm variables.

Product: Primary information on the physiology, growth, and yield of rice in relation to increasing atmospheric $\mathrm{CO}_{2}$.

Approach: The Plant Stress and Protection Research Unit is conducting a long-term study on the effects of increased atmospheric $\mathrm{CO}_{2}$ on physiology, growth and yield of rice at both optimal and stress levels of temperature, water and nutrients. The experiments derive response functions to $\mathrm{CO}_{2}$ concentration under conditions of nonlimiting water and nutrients and nearoptimum temperatures. These results will be used to determine coefficients for specific rate equations to modify and adapt an existing plant-simulation model.

The combined temperature- $\mathrm{CO}_{2}$ effects will be examined for several new cultivars obtained from the International Rice Research Institute. Ancillary measurements of $\mathrm{Ch}_{4}$ emissions in relation to varied environmental conditions (temperature and nutrient status) and physiological status will identify biophysical controls on its release from paddy rice. Companion experiments will investigate interactive effects of $\mathrm{CO}_{2}$ and nitrogen fertilization. An independent modelvalidation experiment is also planned.

Results to Date: A combination of $\mathrm{CO}_{2}$ (330 and 660 $\mathrm{ppm})$ and smooth temperature cycles ( $\max / \min 32 / 23$, $35 / 26$, and $38 / 29^{\circ} \mathrm{C}$ ) were imposed on paddy rice (cv.
IR-72). Aboveground biomass at elevated $\mathrm{CO}_{2}$ was greater for $38 / 29^{\circ} \mathrm{C}(1.47)$ than for $35 / 26^{\circ} \mathrm{C}(1.13)$ or $32 / 23^{\circ} \mathrm{C}$ (1.07). Grain yields were decreased drastically at $38 / 29^{\circ} \mathrm{C}$. Root biomass and root length density were increased noticeably by elevated $\mathrm{CO}_{2}$, and they decreased slightly with increasing temperature. The rootlength-density/root-biomass ratio was similar across all treatments.

Within temperature treatments, leaf $A / C_{1}$ curves were similar at both $\mathrm{CO}_{2}$ concentrations, which implies no photosynthetic acclimation (no down-regulation) to elevated $\mathrm{CO}_{2}$. Previously, the cultivar IR-30 showed down-regulation of Rubisco with increasing $\mathrm{CO}_{2}$.

Rice emitted twice as much methane at $660 \mathrm{ppm}$ and $38 / 29^{\circ} \mathrm{C}$ than at $330 \mathrm{ppm}$ and $32 / 23^{\circ} \mathrm{C}$. Increased root exudation or root mortality could have supplied more substrate for methanogenesis. Rising $\mathrm{CO}_{2}$ and global warming could increase methane emissions from wetlands vegetation:

Leaf sensitivity to $\mathrm{CO}_{2}$ and temperature was added to a canopy-assimilation model. Despite a linear increase in electron transport from 6 to $37^{\circ} \mathrm{C}$, predicted assimilation was near maximum from 24 to $32^{\circ} \mathrm{C}$ at $350 \mathrm{ppm}$ and from 28 to $40^{\circ} \mathrm{C}$ at $700 \mathrm{ppm}$. The broad temperature optimum for canopy assimilation is consistent with our previous measurements.

\section{INTEGRATION OF EXPERIMENTAL AND MODELING APPROACHES TO STUDY COMPETITIVE INTERACTIONS AMONG PLAN'TS UNDER ELEVATED $\mathrm{CO}_{2}$}

BAZZAZ, F. A.

\section{HARVARD UNIVERSITY}

$\begin{array}{ll}\text { FY } 1993 & 280 \\ \text { FY } 1992 & 265 \\ \text { FY } 1991 & 240\end{array}$

04/01/93-03/31/94

Objective: To obtain data for predicting effects of $\mathrm{CO}_{2}$ and physical influences (e.g., light or moisture) with plant communities based on knowledge of component species and their growth, productivity response, and resource use. 
Product: Data on growth and physiology of individuals and mixtures of species at several levels of $\mathrm{CO}_{2}$; parameterizations of responses of individuals and mixtures; and models describing $\mathrm{CO}_{2}$ effects on plant competition in relation to $\mathrm{CO}_{2}$ and other environmental variables.

Approach: Experiments will be carried out with mixtures of species representing annual communities and native grasslands. With $\mathrm{CO}_{2}$-controlled growth chambers, the designs will examine relative responses at three $\mathrm{CO}_{2}$ levels; two light intensities; and various water, nutrient, and temperature conditions. Companion data on physiology will provide insight on causes of differential responses to $\mathrm{CO}_{2}$.

Data will be used in a plant-competition model to simulate $\mathrm{CO}_{2}$ effects on community growth and composition change. The initial phase of the research will develop a database for annuals for which a mechanistic model of interspecific competition will be formulated. Because the model represents general physiological features, it can be tested with other assemblages of species and/or communities (e.g., the general model structure will serve as a basis for application to mixtures of perennial plants subjected to different levels of $\mathrm{CO}_{2}$ and different physical environments).

A supplemental task of the research is to obtain specific data for modeling community response to $\mathrm{CO}_{2}$, temperature, and light. Specifically, experiments will characterize the physiological properties of individual leaves throughout the canopy and the life-cycle of cooccurring plant species. Growth and resource deployment will be examined as well as $\mathrm{CO}_{2}$ and light conditions associated with photosynthesis.

Results to Date: Our studies on the growth responses of six co-occurring temperate forest tree species exposed to various combinations of atmospheric $\mathrm{CO}_{2}$, nutrient, and light levels reached its third year. The species investigated included; gray birch (Betula populifolia Marsh.), white ash (Fraxinus americana L.), red maple (Acer rubrum L.), red oak (Quercus rubra L.), yellow birch (Betula alleghaniensis Britt.), and striped maple (Acer pensylvanicum L.). After 3 years of growth, elevated- $\mathrm{CO}_{2}$-grown plants were significantly larger than ambient $\mathrm{CO}_{2}$-grown plants in only 5 out of 24 cases. Additionally, $\mathrm{CO}_{2}$-induced growth enhancement of the six species declined at different rates during years two and three. Despite increases in photosynthetic rates, decreases in the degree of $\mathrm{CO}_{2}$-induced growth enhancement during years two and three resulted from increased respiratory costs of larger plants, changed biomass allocation, and a shorter growing season. The results suggest that forest-productivity enhancements may not be sustained for long periods of time. Thus, predicted rises in carbon sequestering in terrestrial ecosystems may be less than anticipated. Furthermore, species' differential growth responses to elevated $\mathrm{CO}_{2}$ could result in compositional changes in forests. This could result in additional feedback on forest productivity and the global carbon cycle.

\section{A FIELD STUDY OF THE EFFECTS OF ELEVATED AMBIENT $\mathrm{CO}_{2}$ ON ECOSYSTEM PROCESSES IN CHESAPEAKE BAY WETLANDS}

DRAKE, BERT G.

SMITHSONIAN INSTITUTION

$\begin{array}{lll}\text { FY } 1993 & 249 \\ \text { FY } 1992 & 240 \\ \text { FY } 1991 & 251\end{array}$

09/20/93-09/19/94

Objective: To determine whether elevated ambient $\mathrm{CO}_{2}$ concentration will affect ecosystem production through increased photosynthesis and reduced respiration, alter species composition, alter relative partitioning of biomass between above- and below-ground structures, change carbon and nitrogen concentrations in plant tissue, alter decomposition rates, and change the water balance in plants and plant communities of a mesohaline salt marsh on Chesapeake Bay.

Product: Mechanistic biophysical information on the response of a wetland plant community continuously exposed to elevated $\mathrm{CO}_{2}$ and a database for modeling the effects of $\mathrm{CO}_{2}$ enrichment on ecosystem processes.

Approach: Long-term research will be continued at the Smithsonian Environmental Research Center, a saltmarsh research site near Edgewater, Maryland. Three community types will be investigated in the field, where elevated $\mathrm{CO}_{2}$ levels will be controlled by small, replicated, open-top chambers.

Acclimation of carbon metabolism and light harvesting to $\mathrm{CO}_{2}$ will be measured: 
- Gas-exchange measurements will be made in situ on attached leaves to determine responses to light, $\mathrm{CO}_{2}$, and temperature of plants grown in normal ambient and elevated $\mathrm{CO}_{2}$.

- Rubisco content and activity will be determined as well as other key enzymes and metabolites of the carbon-reduction cycle.

- Photosynthetic electron transport will be measured by fluorescence to determine whether acclimation to elevated $\mathrm{CO}_{2}$ increases the plant's capacity for light harvesting.

The effects of elevated $\mathrm{CO}_{2}$ concentration on ecosystem processes will be measured with nondestructive methods to the extent possible:

- Net ecosystem $\mathrm{CO}_{2}$ and $\mathrm{H}_{2} \mathrm{O}$ exchange will be determined by using the chamber as an open gasexchange cuvette.

- Biomass production and competition will be determined by a census of shoots and allometric relationships between length and weight and by studying the regrowth of roots into cores.

- Decomposition will be followed in situ with litter bags.

- Tissue analysis for nitrogen, carbon, and other molecular constituents will be used to study nutrient balance.

Results to Date: Data were obtained on the activities of several key enzymes in the respiratory electron-transport chain. Elevated $\mathrm{CO}_{2}$ has a direct, immediate effect on the respiration rate of shoots of the sedge Scirpus olneyi and on the leaves of the grass Spartina patens and the shrub Lindera benzoin. The content of the carboxylating enzyme Rubisco is reduced by the elevated $\mathrm{CO}_{2}$ treatments in the sedge Scirpus olneyi and the shrub Lindera benzoin. The transpiration of the understory shrub Lindera benzoin is greatly stimulated by elevated $\mathrm{CO}_{2}$, and this effect translates into increased growth. A pilot study of the effects of elevated $\mathrm{CO}_{2}$ on a scrub oak, palmetto palm ecosystem was initiated in Cape Canaveral, Fla. The growth of the two shrubs Quercus geminata and $Q$. myrtifolia was stimulated by the elevated $\mathrm{CO}_{2}$ during the first year of treatment. In addition, elevated $\mathrm{CO}_{2}$ reduced the rate of dark respiration as well as the protein concentration of these species. These results are all consistent in showing a strong and pervasive effect of chronic elevated $\mathrm{CO}_{2}$ exposure on carbon, nutrient, and water balances of these wild species from three very different ecosystems.

\section{FREE-AIR CARBON DIOXIDE FIELD ENRICHMENT (FACE) FACILITY DEVELOPMENT}

HENDREY, GEORGE

\section{BROOKHAVEN NATIONAL LABORATORY}

$\begin{array}{lrr}\text { FY } 1993 & 1100 \\ \text { FY } 1992 & 935 \\ \text { FY } 1991 & 973\end{array}$

$10 / 01 / 92-09 / 30 / 93$

Objective: To evaluate FACE systems able to maintain stable $\mathrm{CO}_{2}$ concentrations for crops, forests, or other ecosystems; to produce reliable field data about the effects of $\mathrm{CO}_{2}$ enrichment on plants for use in mechanistic plant-growth models; and to evaluate global $\mathrm{CO}_{2}$ fluxes and carbon balances between vegetation and the atmosphere under conditions of elevated atmospheric $\mathrm{CO}_{2}$.

Product: An operational FACE user facility; analyses of FACE system performance; and data on the effects of $\mathrm{CO}_{2}$ enrichment on plants.

Approach: The FACE Program is a cooperative research effort of DOE/OHER and the USDA Agricultural Research Service in which USDA/ARS supports field research on crop-plant responses to $\mathrm{CO}_{2}$ enrichment and development of the $\mathrm{CO}_{2}$ response models. The DOE component emphasizes FACE facility engineering and operations and basic research on mechanisms of plant responses to $\mathrm{CO}_{2}$ enrichment. Phase 1 of the DOE/ OHER FACE Program, completed in 1987, developed a functional FACE system that could reliably centrol $\mathrm{CO}_{2}$ to $\pm 20 \%$ of an increment $200 \mu \mathrm{mol} / \mathrm{mol}$ over ambient more than $98 \%$ of the time at the center of the plot. Over plots $>130 \mathrm{~m}^{2}$, average $\mathrm{CO}_{2}$ concentration varied by about 5\%. Phase $2(1987-1989)$ developed a FACE User Facility within which many investigators could participate in complex, integrated field experiments to evaluate $\mathrm{CO}_{2}$-enrichment effects on cotton. Phase 3 (1990-1993) will execute full-scale field experiments on monocultures in a crop setting. Phase 4 will develop FACE systems for other types of ecosys- 
tems, including forests. This will lead to Phase 5, fullscale experimental $\mathrm{CO}_{2}$ enrichment of a forest with concomitant studies of the biological responses and mechanisms governing its responses.

Results to Date: The effects of elevated $\mathrm{CO}_{2}$ on winter wheat at all levels of plant organization were studied, from canopy photosynthesis to feedback controls on the expression of genes regulating proteins associated with photosynthesis by 37 investigators from 7 nations and 21 research units. Additional scientific effort in FY 1993-1994 will be directed to evaluating and reporting results from the wheat experiment. Brookhaven National Laboratory is currently developing similar FACE facilities in other types of ecosystems in the United States and abroad. A FACE facility was completed and began operation at the Swiss Federal Institute of Technology, Zurich.

\section{ELEVATED $\mathrm{CO}_{2}:$ MODELING COTTON RESPONSE INTERACTIONS WITH TEMPERATURE ON PHOTOSYNTHESIS, INSECT-POPULATION DYNAMICS, AND TEMPERATURE INTERACTIONS AND LONG-TERM EFFECTS ON TREES}

\section{KIMBALL, B. \\ WATER CONSERVATION LABORATORY and WESTERN COTTON RESEARCH LABORATORY}

$\begin{array}{lr}\text { FY } 1993 & 110 \\ \text { FY } 1992 & 0 \\ \text { FY } 1991 & 0\end{array}$

09/01/93-08/31/94

Objective: To assemble data for modifying a plantgrowth simulation model, to determine the effects of elevated $\mathrm{CO}_{2}$ on the growth and physiology of trees, to determine the effects of $\mathrm{CO}_{2}$ and elevated temperature on feedback inhibition of photosynthesis and on rates of development and growth of plant organs, and to determine if beet armyworm and pink bollworm are affected by $\mathrm{CO}_{2}$-altered tissue of plants.

Product: Primary information on the physiology, growth, and yield of plants, trees, and annual species under increasing atmospheric $\mathrm{CO}_{2}$.

Approach: Taking advantage of full sunlight conditions at Phoenix, Ariz., and using temperature- and $\mathrm{CO}_{2}$ - controlled greenhouses, cotton studies will be conducted at two temperatures and two $\mathrm{CO}_{2}$ concentrations. Switching experiments will examine the occurrence of feedback inhibition on photosynthesis, where supply of assimilates (related to elevated $\mathrm{CO}_{2}$ and increased carbon fixation) may exceed demand, causing a decrease in photosynthetic capacity. When inhibition is observed, additional measurements will investigate intercellular phosphate and $\mathrm{O}_{2}$ insensitivity of Rubisco.

The open-top $\mathrm{CO}_{2}$-enrichment chamber technique will be used with ambient and 650-ppm levels of $\mathrm{CO}_{2}$, and plant physiology and growth will be determined for different temperature conditions. An experiment with citrus will collect data on the $\mathrm{CO}_{2}$ effects on leaf area, biomass, yield, foliage temperatures, net photosynthesis, stomatal conductance, photosynthetic response to $\mathrm{CO}_{2}$, leaf carbohydrate content, and stomatal response at variable soil-moisture content.

Because $\mathrm{CO}_{2}$ enrichment changes the biochemical properties of plant tissue, which in turn influences the feeding habits and rates of foraging of insects, the cotton experiment will furnish plant material for an ancillary experiment to determine how host cotton grown at two levels of $\mathrm{CO}_{2}$ and two temperatures affects the growth of beet armyworm.

Results to Date: Orarge trees in open-top chambers enriched by $300 \mathrm{ppm}$ of $\mathrm{CO}_{2}$ above ambient continue to grow nearly three times as fast as the control trees after more than 5 years of enrichment. The ratio of fruit production of the enriched trees to that of the ambient trees was 25, 7.5, and 3.6 for 1990, 1991, and 1992, respectively. This fruit-production ratio is approaching the enriched/ambient biomass ratio of 2.8. Fine-root biomass in the upper $0.4 \mathrm{~m}$ of the soil profile was measured at 2-month intervals and found to be increased 2.3 times by $\mathrm{CO}_{2}$ enrichment. Leaf dry weight was also measured at 2-month intervals, and it was unaffected by $\mathrm{CO}_{2}$ enrichment in the winter but was increased up to $40 \%$ in the summer. Considerable progress was made in documenting the cotton growth model $\mathrm{COTCO} 2$, which is being developed to predict the effect of the increasing atmospheric $\mathrm{CO}_{2}$ concentration and changing climate variables on cotton growth and water use. Audio tapes, flow charts, and a manuscript were produced.

In addition, an initial working version of WHEAT was written. Similar to COTCO2, WHEAT is a detailed plant-growth model for predicting the impact of global change on wheat growth and water use. 


\section{HERBIVORE RESPONSES TO PLANTS GROWN IN ENRICHED $\mathrm{CO}_{2}$ ATMOSPHERE}

\author{
LINCOLN, DAVID E.
}

UNIVERSITY OF SOUTH CAROLINA

$\begin{array}{lr}\text { FY } 1993 & 70 \\ \text { FY 1992 } & 71 \\ \text { FY 1991 } & 0\end{array}$

$04 / 01 / 93-02 / 28 / 94$

Objective: To measure both response of host plants and insect herbivores to $\mathrm{CO}_{2}$, with an emphasis on changes of insect consumption and consequent impacts on plants.

Product: Experimental data on insect feeding rates as affected by $\mathrm{CO}_{2}$-altered nutritive and biochemical properties of plant tissue.

Approach: The response of sawfly to loblolly pine needle biochemistry when the plants are field-grown in elevated $\mathrm{CO}_{2}$ will be studied. The pine-sawfly system will permit an experimental test of the hypothesis that the higher leaf carbon content in elevated $\mathrm{CO}_{2}$ is a primary determinant of altered insect performance.

Experimental manipulation of the nutrient supply to pine and poplar trees will be used to evaluate insect larval performance at different carbohydrate-to-protein (i.e., different $C / N$ ) ratios of leaves.

As $\mathrm{CO}_{2}$ treatment changes carbon source/sink relationships and the formation of plant biochemicals, detailed biochemical and molecular methods will be employed to determine chalcone synthase activity, mRNA levels, and flavonoid and tannin content. Molecular studies of chalcone synthase are proposed because it is a key regulatory enzyme of flavonoid biosynthesis, which subsequently controls the level of the alleochemicals, such as phenolics and terpenes, in leaves.

Results to Date: Young larvae, which are often the most sensitive to changes in host-plant characteristics, of a native sawfly responded to loblolly pine needles in a manner similar to the previously studied responses of older lepidopteran larvae to herbaceous plants. Feeding increased under elevated $\mathrm{CO}_{2}$ treatments $(+150$ and $+300 \mathrm{ppm}$ ), while larval growth was little changed. Leaf nutritional quality ( $\mathrm{C} / \mathrm{N}$ ratio) changed under elevated
$\mathrm{CO}_{2}$ growth conditions as leaf starch content increased and leaf protein content decreased. The insects responded with increased feeding rate and increased nitrogen-use efficiency as presumed compensatory responses to the reduction in leaf nitrogen availability at elevated $\mathrm{CO}_{2}$. One of the major volatile monoterpenes of the pine needles, beta pinene, declined under elevated $\mathrm{CO}_{2}$ growth conditions. For this insect, which has several generations during each growing season, springfeeding larvae used needles with higher starch and lower protein and fed at a higher rate compared to a fallfeeding larvae.

\section{IGPB-GCTE SYMPOSIUM ON TERRESTRIAL ECOSYSTEM RESPONSE TO ELEVATED $\mathrm{CO}_{2}$ TO BE HELD OCTOBER 18-21, 1993}

$$
\text { MOONEY, HAROLD A. }
$$

STANFORD UNIVERSITY

$\begin{array}{lr}\text { FY } 1993 & 25 \\ \text { FY } 1992 & 0 \\ \text { FY } 1991 & 0\end{array}$

09/15/93-09/14/94

Objective: To conduct an International Sy mposium on the Response of Terrestrial Ecosystems to Elevated $\mathrm{CO}_{2}$ for the IGBP Core Project on Global Change and Terrestrial Ecosystems (GCTE).

Product: The identification of what is known and uncertain about the effects of elevated $\mathrm{CO}_{2}$ and changing climate variables on whole ecosystems, a strategy for experimental research to effectively address the unresolved scientific uncertainties, and a framework for coordinated experiments by participants in the international Global Change and Terrestrial Ecosystem Program.

Approach: The Symposium will solicit participation by a broad range of intellectual and institutional groups, about half from Europe and half from the United States. This mix of expertise will synthesize existing knowledge and exchange ideas on how to organize and implement future res_arch with representative ecosystems on a global scale. 


\section{$\mathrm{CO}_{2}$ EXCHANGE, ENVIRONMENTAL PRODUCTIVITY INDICES, AND \\ PRODUCTIVITY OF AGAVE AND CACTI \\ UNDER CUR'RENT AND ELEVATED ATMOSPHERIC: $\mathrm{CO}_{2}$ CONCENTRATIONS}

\author{
NOBEL, PARK S. \\ UNIVERSITY OF CALIFORNIA AT LOS \\ ANGELES
}

$\begin{array}{ll}\text { FY 1993 } & 125 \\ \text { FY 1992 } & 125 \\ \text { FY 1991 } & 133\end{array}$

09/20/93-09/19/94

Objective: To examine the influence of elevated $\mathrm{CO}_{2}$ concentrations and accompanying environmental changes on net $\mathrm{CO}_{2}$ uptake and productivity of crassulacean acid metabolism (CAM) plants; to use the environmental productivity index (EPI) to evaluate plant response to the $\mathrm{CO}_{2}$ enrichment in relation to soil water status, air temperature, and photosynthetic photon flux density; and to examine the molecular basis for interpreting $\mathrm{CO}_{2}$ effects on CAM-plant metabolism.

Product: Fundamental information on $\mathrm{CO}_{2}$ fixation by the CAM pathway, which approaches the upper bound of $\mathrm{CO}_{2}$ fixation by vascular plants.

Approach: Experiments with Opuntia will investigate the dependence of $\mathrm{CO}_{2}$ exchange on plant age, and measurements of both transpiration and net $\mathrm{CO}_{2}$ exchange will determine water-use efficiency. The study will determine if the EPI holds for plants grown at elevated $\mathrm{CO}_{2}$. The $\mathrm{CO}_{2}$-enrichment experiments will be conducted outdoors in open-top contrcl chambers to take advantage of natural lighting and in situ conditions for growing plants. Also, some plants will be grown under controlled conditions.

Molecular studies will involve the energy costs and molecular efficiency of the $\mathrm{CO}_{2}$-fixation process at ambient and elevated $\mathrm{CO}_{2}$ conditions. Fundamental energy requirements will be worked out for two enzyme pathways [ribulose-1,5-bisphosphate (Rubisco) and phosphenol-pyruvate carboxylase (PEPCase)] that fix $\mathrm{CO}_{2}$ and form carbohydrate molecules differently.
The metabolic pathways will be investigated by $\mathrm{CO}_{2}$ gas exchange and by using carbon-isotope fractionation, which is becoming the favored way of determining the relative importance of different pathways of carbon metabolism. Metabolites of the initial step of $\mathrm{CO}_{2}$ fixation have different $\Delta^{13} \mathrm{C}$ values, which provides a measure of relative enzyme intensity for different metabolic pathways, conditions, and time periods.

Results to Date: Environmental responses of the CAM species Opuntia ficus-indica were studied at various $\mathrm{CO}_{2}$ concentrations in open-top chambers in the field at the University of California, Riverside, for 23 weeks. After 9 weeks of growth, daily net $\mathrm{CO}_{2}$ uptake at $\mathbf{5 2 0}$ and $720 \mu \mathrm{L} / \mathrm{L} \mathrm{CO}_{2}$ averaged $44 \%$ and $71 \%$ higher, respectively, than that at $370 \mu \mathrm{L} / \mathrm{L} \quad \mathrm{CO}_{2}$. These increases are unexpectedly large stimulations of net $\mathrm{CO}_{2}$ uptake caused by raising the ambient $\mathrm{CO}_{2}$ level. Although the chlorophyll content was $19 \%$ and $62 \%$ lower, respectively, plant biomass production over 23 weeks in 520 and $720 \mu \mathrm{L} / \mathrm{L} \mathrm{CO}_{2}$ was $21 \%$ and $55 \%$ higher than that at $370 \mu \mathrm{L} / \mathrm{L} \mathrm{CO}_{2}$.

The effects of current $(380 \mu \mathrm{L} / \mathrm{L})$ and elevated $(740$ $\mu \mathrm{L} / \mathrm{L}) \mathrm{CO}_{2}$ concentrations were also determined for $O$. ficus-indica grown in pots for 18 weeks in environmentally controlled rooms. Dry-weight gain was enhanced $24 \%$ by elevated $\mathrm{CO}_{2}$ during the first 10 weeks but only $8 \%$ for 18 weeks. Increasing the soil volume by a factor of 10 led to a greater stimulation of daily net $\mathrm{CO}_{2}$ uptake and biomass production than did doubling the $\mathrm{CO}_{2}$ level. The amount of cladode nitrogen per unit dry weight decreased as the $\mathrm{CO}_{2}$ level was raised and increased as pot size was increased.

\section{ELEVATED CARBON DIOXIDE EFFECTS ON WOODY-PLANT SOIL SYSTEMS}

NORBY, R. J.; LUXMOORE, R. J.; and O'NEILL, E.

OAK RIDGE NATIONAL LABORATORY

$\begin{array}{ll}\text { FY } 1993 & 400 \\ \text { FY } 1992 & 400 \\ \text { FY } 1991 & 487\end{array}$

$10 / 01 / 92-09 / 30 / 93$ 
Objective: To determine whether the short-term responses of temperate forest trees to elevated concentrations of $\mathrm{CO}_{2}$ are sustained over several growing seasons under field conditions and to provide the physiological basis for tree-growth and forestecosystem response.

Product: Data on the effects of $\mathrm{CO}_{2}$ on growth and physiological responses of white oak and yellow poplar saplings under field conditions, providing insights into larger-scale ecologically relevant responses, including litter quality, decomposition, below-ground responses, and nutrient relations.

Approach: White oak and yellow poplar seedlings will be planted in the ground in open-top field chambers. The chamber atmospheres will be continuously maintained at ambient $\mathrm{CO}_{2}$, ambient $+150 \mathrm{ppm}$, or ambient $+300 \mathrm{ppm}$ during the growing seasons. Above-ground growth and leaf production will be measured. Physiological measurements will include photosynthesis, seasonal and instantaneous water-use efficiency, leaf respiration, stem respiration, and carbohydrate concentrations. After three or four growing seasons, the trees will be harvested, and the dry mass and nitrogen concentrations of the above- and below-ground components will be determined. Leaf litter will be collected in the fall and analyzed for components that predict rate of litter decomposition (lignin, cellulose, nitrogen, and phenolics). Litter will also be placed in mesh bags, and the rate of decomposition and nitrogen release will be measured.

Results to Date: White oak saplings were harvested at the end of their fourth growing season in the field in $\mathrm{CO}_{2}$-enriched atmospheres. Whole-tree dry mass was $59 \%$ greater in $+150 \mathrm{ppm}$ and $134 \%$ greater in +300 , compared to ambient-grown trees. The effects of $\mathrm{CO}_{2}$ on root growth were proportionately larger, and the effects on leaf production were proportionately smaller. Most of the large stimulation in growth by $\mathrm{CO}_{2}$ occurred during the first several months of the experiment; subsequent relative growth rates were similar in all treatments, although stem production per unit leaf area was higher in elevated $\mathrm{CO}_{2}$. Fine-root density also was higher in elevated $\mathrm{CO}_{2}$, and this was associated with increased $\mathrm{CO}_{2}$ efflux from the soil.

A review of simulators of forest responses to $\mathrm{CO}_{2}$ enrichment showed some similarities and contrasts between models with differing scales of resolution. The stochastic effects of mortality and ingrowth in succession models introduced variability that masked the $\mathrm{CO}_{2}$ effect.

Soil warming has been identified as a significant factor in global-change modeling. Any increase in mean annual air temperature will result in a similar increase in temperature of the root zone of vegetation. Increases in soil respiration and in nutrient mineralization can lead to positive or negative feedback, respectively, to global warming.

\section{RESPONSE OF TUNDRA ECOSYSTEMS TO ELEVATED ATMOSPHERIC $\mathrm{CO}_{2}$}

OECHEL, WALTER C.

\section{SAN DIEGO STATE UNIVERSITY}

$\begin{array}{ll}\text { FY } 1993 & 300 \\ \text { FY } 1992 & 300 \\ \text { FY 1991 } & 300\end{array}$

09/15/93-09/14/94

Objective: To determine current patterns of $\mathrm{CO}_{2}$ flux from tussock and wet coastal tundras along environmental gradients on the North Slope of Alaska at sites representing a range of vegetation types and environmental conditions, to evaluate recent patterns of peat accumulation in tussock and wet coastal tundras, and to determine methane content of permafrost and current rates of methane release as a function of vegetation and environment.

Product: A definitive statement of what is known and unknown about tundra response to varying $\mathrm{CO}_{2}$ and climate conditions, including measurements of $\mathrm{CO}_{2}$ and $\mathrm{CH}_{4}$ exchange across tundra ecosystems.

Approach: Current rates of $\mathrm{CO}_{2}$ and $\mathrm{CH}_{4}$ flux along a latitudinal gradient from the foothills of the Brooks Range to the coast of the Arctic Ocean will be evaluated. Measurements will be made along the transect formed by the Haul Road and at other sites where permafrost-temperature research has been done on the North Slope. Peat-profile dating will be performed at the same sites as the $\mathrm{CO}_{2} / \mathrm{CH}_{4}$ flux measurements to compare past and current rates of carbon accumulation or loss.

Field research will be carried out with small pop-on chambers to measure $\mathrm{CO}_{2}$ and $\mathrm{CH}_{4}$ fluxes with different 
ecosystems across the North Slope of Alaska. Simultaneously, environmental parameters (air temperature, radiation, relative humidity, soil moisture, and temperature) will be determined during the short-term gasexchange experiments. In addition, continuous monitoring of the physical environment will be done at three sites. Ecosystem data (leaf area, aboveground biomass, carbon content of the thaw layer, and depth to permafrost) will be determined simultaneously with the flux measurements. Initial interpretations will relate $\mathrm{CO}_{2}$ and $\mathrm{CH}_{4}$ fluxes to environmental and biotic properties of the systems under observation.

Results to Date: The U.S. International Biophysical Program (IBP) Site 2 at Barrow Alaska was revisited, and $\mathrm{CO}_{2}$ flux measurements were made for comparison with those made during the early seventies. $\mathrm{CO}_{2}$ flux was measured throughout the 1993 growing season at IBP Site 2 and at Central Marsh. The latter site is near the NOAA Barrow observatory and the proposed DOE ARM and NSF Arctic System Science Program (ARCSS) sites. Preliminary measurements show good agreement between tower methods (aerodynamic and eddy-correlation methods) and chamber methods. Both methods of determining $\mathrm{CO}_{2}$ flux indicate a marked change in net $\mathrm{CO}_{2}$ flux from the strong sink recorded during the U.S. IBP to a weak source to the atmosphere in 1993. These measurements support other recent findings that the Alaskan tundra may have shifted from a $\mathrm{CO}_{2}$ sink to a source within the past few decades. Aircraft trace-gas flux measurements were scheduled for the end of the 1993 season to determine trace-gas flux over an area extending from the Brooks Range into the Arctic Ocean and from Barrow to as far as the Alaskan Yaunal Wildlife Refuge. $\mathrm{CO}_{2}$ flux measurements derived from aircraft measurements will be compared with those derived from the tower and chamber approaches. This project involves participation with National Institute of Agro-Environmental Sciences, Tsukuba City, Japan, NOAA, NSF, and ORNL.

Effects of altered moisture and temperature on $\mathrm{CO}_{2}$ and $\mathrm{CH}_{4}$ flux have been measured along the Haul Road at Prudhoe Bay and Toolik Lake, Alaska.

In addition, $\mathrm{CO}_{2}$ flux measurements were made on the Taimyr Peninsula at past Russian IBP sites and in the Kolyma lowlands on sites where there is ongoing Russian trace-gas flux research.

Effects of long-term growth and development at elevated $\mathrm{CO}_{2}$ was investigated at a cold $\mathrm{CO}_{2}$ spring near Olafsvik, Iceland. Initial results indicate that there may be a long-term response spanning centuries to millennia. Emphases in 1993 were on the long-term structural, physiological, chemical, and biochemical adjustment to elevated $\mathrm{CO}_{2}$.

\section{RANGELAND-PLANT RESPONSE TO ELEVATED $\mathrm{CO}_{2}$}

OWENSBY, CLENTON E.

\section{KANSAS STATE UNIVERSITY}

$\begin{array}{ll}\text { FY } 1993 & 260 \\ \text { FY } 1992 & 262 \\ \text { FY } 1991 & 292\end{array}$

$$
09 / 15 / 93-09 / 14 / 94
$$

Objective: To determine the extent to which the C4dominated grassland will sequester carbon and act as a buffer against future increases in atmospheric $\mathrm{CO}_{2}$.

Product: Data on the physiology and plant growth of representative species of the grassland and measurements of carbon fixation and below-ground productivity to provide the basis for estimates of carbon transformation into soil humus.

Approach: Experiments will be conducted in situ in undisturbed prairie. Aboveground biomass and growth rate will be determined by clipping and separating plant material by species and component parts. Below-ground productivity will be determined from root ingrowth into buried sand bags.

Carbon turnover, microbial metabolism, and carbon source/sink relationships will be estimated from measures of soil respiration and decomposition of organic residues (plant and microbial material). Microbial biomass will be determined by fumigationincubation techniques, and soil respiration will be measured by trapping soil-generated $\mathrm{CO}_{2}$.

Carbon fixatio : and water flux will be measured for the intact whole canopy as well as for individual leaves. Leaf-level photosynthesis will be measured in the field experiment on tillers at three canopy heights and at controlled light and temperatures.

Isotopic tracer techniques and the CENTURY model will be used in studies of soil-organic-matter dynamics 
and for specific estimates of net carbon retention in soil. These data, coupled with observations of $\mathrm{CO}_{2}$ fixation rates by plants, will provide generic information on the carbon balance of grassland ecosystems and on their possible influence on the sequestering of atmospheric $\mathrm{CO}_{2}$.

Results to Date: Elevated $\mathrm{CO}_{2}$ resulted in increased above- and below-ground biomass production through improved water-use efficiency. $\mathrm{C} 4$ perennial grass dominants have remained relatively constant in the stand, while C3 grasses have declined and C3 forbs increased. After 4 years, soil carbon and microbial biomass have increased under elevated $\mathrm{CO}_{2}$. Nitrogen concentration in above- and below-ground biomass was reduced under elevated $\mathrm{CO}_{2}$ compared to ambient. Total $N$ and $P$ in above- and below-ground biomass was greater under elevated $\mathrm{CO}_{2}$ than ambient. Fiber components increased, and rumen microbial digestion decreased for hand-clipped and esophageally-fistulatedsheep-collected samples under elevated $\mathrm{CO}_{2}$ compared to ambient. The rate of surface-litter disappearance did not vary among treatments, but C3 litter disappeared at a faster rate than did $\mathrm{C} 4$ litter. Midday xylem pressure potentials for Andropogon gerardii under elevated $\mathrm{CO}_{2}$ indicated reduced water use compared to ambient. Stomatal response to sun/shade events is faster under elevated $\mathrm{CO}_{2}$ than with ambient $\mathrm{CO}_{2}$, thereby further reducing water loss for $\mathrm{CO}_{2}$-enriched plants. Photosynthetic response of plants exposed to ambient and elevated $\mathrm{CO}_{2}$ indicated that in years with moisture stress the photosynthetic potential of $A$. gerardii is greater for plants grown under high $\mathrm{CO}_{2}$ than those grown under ambient $\mathrm{CO}_{2}$.

\section{MODELING THE RESPONSE OF PLANTS AND ECOSYSTEMS TO $\mathrm{CO}_{2}$ AND CLIMATE CHANGE}

REYNOLDS, JAMES F.

\section{DUKE UNIVERSITY}

$\begin{array}{lr}\text { FY 1993 } & 425 \\ \text { FY 1992 } & 400 \\ \text { FY 1991 } & 0\end{array}$

09/20/93-09/19/94

Objective: To construct generic models of leaf (GENLEAF), canopy (CANDO), and whole-plant response (ALLOC) to elevated $\mathrm{CO}_{2}$ and climate change; to incorporate models of plant responses to elevated $\mathrm{CO}_{2}$ into a generic ecosystem model (SERECO); to study the effects of change in $\mathrm{CO}_{2}$ and climate at regional and global scales; to validate model predictions of whole-plant and total ecosystem response against field studies; and to test specific hypotheses and to make predictions about primary, secondary, and tertiary effects of elevated $\mathrm{CO}_{2}$ and climate change on individual plants and ecosystems.

Product: A suite of plant and ecosystem models that will analyze the combined effects of $\mathrm{CO}_{2}$ and changing climate conditions.

Approach: The GENLEAF module will be refined and tested and will focus on nitrogen, phosphorus controls on photosynthesis at high $\mathrm{CO}_{2}$, carbon source/sink feedback effects on photosynthesis (particularly shifts in the $A / C_{i}$ curves), and carbon metabolism of different plant tissues as influenced by elevated temperature. Formulations of stomatal conductance will be validated.

CANDO will be modified to treat effects of water stress, leaf age, and acclimation on photosynthesis. Detailed data on canopy architecture from the tall-grass prairie experiments will be used as a test of light microclimate, and 1-D radiation-transfer schemes will be compared with 3-D schemes. Differences between these schemes will be evaluated to determine adequacy for application with different canopies having substantially different architectures. Problems of scaling leaf-level photosynthesis to the canopy of loblolly pine will be examined.

For the ALLOC routine, which describes carbon and nutrient allocation patterns for leaf and stem tissue, research and modeling will be pursued for mechanistic explanations for above- vs below-ground allocation of carbohydrate and nutrient material in plants.

SERECO will be further validated with experimental data from the grassland experiments at Kansas State University and from planned free-air- $\mathrm{CO}_{2}$-exchange (FACE) experiments with loblolly pine at the Duke Forest. Both types of experiments provide ecosystemlevel observations suitable for patch-scale validation.

GENDEC will be further developed with results from several experiments now under way.

Results to Date: We have completed the prototype version of GePSi, a modular plant-growth model that 
includes leaf photosynthesis, canopy architecture, root architecture, and root nutrient and water uptake. The model successfully predicts growth responses of loblolly pines under different $\mathrm{CO}_{2}$ environments. A study of the effects of elevated $\mathrm{CO}_{2}$ on the root morphology and growth in loblolly pine showed that the main effects of $\mathrm{CO}_{2}$ are in morphological changes in the roots; also, high $\mathrm{CO}_{2}$ may exacerbate nutrient stress under conditions of low nitrogen.

At the ecosystem level, we completed development of a generic model of decomposition and nutrient availability that is mechanistic in terms of microbial metabolism and nutrient release. To test the model predictions, an experimental study was conducted of decomposition of tallgrass prairie leaf litter produced under different $\mathrm{CO}_{2}$ levels both with and without nitrogen fertilization. Elevated $\mathrm{CO}_{2}$ produces little change in leaf carbon chemistry or nitrogen concentration, and consequently, little difference in decomposition over 18 months. However, the decomposition of litter from different species did show a difference, suggesting that a possible secondary effect of $\mathrm{CO}_{2}$ on decomposition may result from changes in community composition.

Experiments showed that high root temperatures enhance the positive response to high $\mathrm{CO}_{2}$ in two shrub species, and the combination of factors can make them very strong competitors in the arid grasslands that they frequently invade. They also showed that elevated $\mathrm{CO}_{2}$ enhances leaf area of a C4-grass-dominated prairie but does not alter species composition or greatly change the distribution of leaves within the canopy.

\section{INTEGRATION OF EXPERIMENTAL AND MODELING APPROACHES IN THE UNMANAGED-ECOSYSTEM RESEARCH PLAN}

\author{
STRAIN, BOYD R. \\ DUKE UNIVERSITY

$\begin{array}{lll}\text { FY } 1993 & 429 \\ \text { FY 1992 } & 484 \\ \text { FY 1991 } & 451\end{array}$

$09 / 15 / 93-09 / 14 / 94$

Objective: To examine the direct physiological effects of $\mathrm{CO}_{2}$ on plants with an emphasis on the long-term effects on the physiology, growth, and ecosystem interactions of loblolly pine.

Product: Data for modeling and predicting changes of plant physiology and growth as a function of different future $\mathrm{CO}_{2}$ and climate conditions, including new experimental information on physiological and ecological controls of whole-plant and whole-system functions.

Approach: Under field conditions, physiological controls on plant response to $\mathrm{CO}_{2}$ will be examined with special attention to feedback effects of the plant source-sink carbon balance on $\mathrm{CO}_{2}$ assimilation rate.

A 2-year partial-factorial-design experiment will be conducted with seedlings and saplings to determine if limited nitrogen and phosphorus affects loblolly pine's response to increased $\mathrm{CO}_{2}$. Gas exchange (in terms of the $A / C_{i}$ curve), whole-plant carbon balance, chlorophyll fluorescence, leaf-tissue composition (for $\mathrm{CHO}$, proteins, and nutrients), and Rubisco activity will be determined as functions of $\mathrm{CO}_{2}$ treatment.

These studies will be continued for an additional 3 years to achieve long-term data on plant response to increased concentrations of atmospheric $\mathrm{CO}_{2}$. Measurements will continue with plants that have been conditioned by high $\mathrm{CO}_{2}$, which will allow a realistic and in-depth examination of the degree and mechanisms of how plants photosynthetically acclimate to $\mathrm{CO}_{2}$. Moreover, plants will be grown in the ground to avoid stresses caused by root confinement.

Existing information about carbon fixation and allocation in plants will also be assembled and used in the evaluation of ecosystem productivity and stability in relation to $\mathrm{CO}_{2}$ and climate variables (including nutrient availability and cycling). It will also be used in an assessment of the properties of the ecosystem likely to be affected by $\mathrm{CO}_{2}$-altered growth (e.g., plant-animal and plant-microbial interactions and water fluxes). This information will be used in reexamining the Farquhar equation and to formulate generic models of whole-plant and whole-system response to $\mathrm{CO}_{2}$ and climate (e.g., PRECO and SERECO).

Results to Date: During 1992 and 1993, loblolly pine trees germinated in April 1991 were maintained in the field in open-topped chambers at ambient and at two enriched- $\mathrm{CO}_{2}$ levels and at variable soil fertility. Physiological measurements (including net photosyn- 
thesis, dark respiration, transpiration, and Rubisco activity and concentration) demonstrated direct $\mathrm{CO}_{2}$ cffects and factor interaction among the soil-nutrient levels and atmospheric $\mathrm{CO}_{2}$. Anatomical, morphological, and tissue-nutrient analyses also indicated significant interactive effects of increased atmospheric $\mathrm{CO}_{2}$ and soil $\mathbf{N}$ and $\mathbf{P}$ on secondary plant-growth responses. Collaboration with scientists from other laboratories and institutions in the modeling of ecological, physiological, and morphological parameters indicates that changing biochemical and growth effects in plants propagate through the other trophic levels to significantly affect herbivores, decomposers, and symbiotic mycorrhizal fungi. Laboratory studies of preindustrial $\mathrm{CO}_{2}$ concentrations suggest that the long periods of the Pleistocene, when $\mathrm{CO}_{2}$ partial pressures were below 30 $\mathrm{Pa}$, were difficult periods for the competitive survival of plants with the $\mathrm{C} 3$ photosynthetic pathway. 


\section{OCEAN RESEARCH}

The oceans have an important role in the Earth's climate system and in determining the fate of natural and anthropogenic $\mathrm{CO}_{2}$ in the atmosphere. For example, the oceans are the largest known sink for anthropogenic $\mathrm{CO}_{2}$. They also could delay detection of increased surface air temperature resulting from $\mathrm{CO}_{2}$, . nissions by storing excess heat. Uncertainties about complex interactions among clouds, oceanic heat, and carbon storage and apparent imbalances between sources and sinks of carbon underscore the need for focused ocean research to accomplish the core goals of the global-carbon and climate research programs.

Initiated in 1989 as part of the expanded programs, ocean research was designed to provide special information for other core research as well as expansion programs, such as ARM and Quantitative Links. The program focuses on understanding critical physical processes that limit the accuracy of ocean-circulation models used for climate prediction and on defining chemical and biological processes that control the amount of anthropogenic $\mathrm{CO}_{2}$ taken up and sequestered by the oceans.

Ocean research has developed a comprehensive approach of laboratory and field measurements, advanced models, new instrumentation, and remote-sensing techniques to provide critical scientific information. The program maintains close coordination with global-change research conducted by other agencies; such as the World Ocean Circulation Experiment (WOCE) and the Joint Global Ocean Flux Study (JGOFS).

Research Objectives: One of the two main program objectives is to improve the treatment of physical processes in ocean models to be used for the prediction of climate at regional scales. By meeting this objective, more-credible and -accurate predictions for evaluating the effects of energy use on the Earth system will be possible. The second objective is to develop a global data set describing the oceanic carbonate system for the calibration and verification of ocean-carbon-cycle models. Progress will lead to a better understanding of the interaction and potential feedbacks between changing climate and carbon cycling in the ocean. Together with carbon-cycle-modeling efforts, accomplishing this objective will provide more accurate predictions of future $\mathrm{CO}_{2}$ levels in the atmosphere.

Research Questions: The ocean-research objectives were developed to answer these questions:

- How is $\mathrm{CO}_{2}$ distributed in the oceans, and how is it transported among sources and sinks'?

- What processes control the rate $\mathrm{CO}_{2}$ exchange between the atmosphere and ocean?

- What inaccuracies in coupled atmosphere-ocean OCMs result from parameterizations of oceanic mixing and convection?

- How can parameterizations of oceanic mixing and convection be improved to reduce $\mathrm{OCM}$ inaccuracies while increasing computational efficiency?

\section{Program Manager}

Michael Riches

Environmental Sciences Division

Department of Energy, ER-74

Washington, DC 20585

(301) 903-3264

Internet:

mike.riches (1) mailgw.er.doe.gov 


\section{Research Summaries}

\section{EFFECTS OF WHITECAPS ON THE AIR/SEA $\mathrm{CO}_{2}$ EXCHANGE}

ASHER, W.E.

\section{BATTELLF MARINE SCIENCES LABORATORY}

$\begin{array}{ll}\text { FY } 1993 & 630 \\ \text { FY } 1992 & 630 \\ \text { FY } 1991 & 530\end{array}$

$10 / 01 / 92-09 / 30 / 93$

Objectlve: To develop a method for prediction of air/sea gas-transport velocities from passive microwave radiometric measurements of oceanic-whitecap coverage.

Product: An empirical parameterization of air/water gas transport velocities in terms of fractional whitecap coverage and molecular diffusivity developed from laboratory experiments; a test of the empirical parameterization with oceanic whitecap coverages and in situ gas-exchange measurements; a relationship between apparent microwave-brightness temperature of the ocean surface and fractional-area whitecap coverage.

Approach: The main goal is to be able to predict the air/sea gas-transport velocity $k_{L}$ from fractional-area whitecap coverage $W_{c}$. Because it may be possible to determine $W_{c}$ from satellite measurements of the seasurface apparent microwave-brightness teniperature, $\sigma$, correlation of $k_{L}$ with $W_{c}$ may allow estimation of $k_{L}$ with the spatial coverage necessary for calculating global $\mathrm{CO}_{2}$ fluxes. To develop this remote-sensingbased method for determining $k_{L}$, Battelle's whitecap simulation tank (WST) will be used to develop an empirical model for calculating $k_{L}$ from bubble-plume coverage, $B_{c}$; molecular diffusivity, $D_{;}$and aqueousphase gas solubility, $\alpha$. Study of the gases $\mathrm{CO}_{2}$, oxygen, helium, sulfur hexafluoride, and dimethyl sulfide will allow parameterization of $k_{L}$ in terms of $B_{c}, D$, and $\alpha$. A laser-phased Doppler anemometer (LPDA) will be used to measure turbulence and bubble populations in the WST. The LPDA data will be used for developing the physical basis for empirical models relating $k_{L}$ and $B_{c}$. This effort will aid in applying the WST parameterization to oceanic conditions.
Field experiments will be conducted to develop the relation between $W_{c}$ and $\sigma$. A field experiment to study the effect of bubble plumes on the microwave emissivity of the sea surface on Sequim Bay, Washington, provided data needed to verify field results obtained quring the Nantucket Shoals field experiment completed in March 1991. A pilot outdoor wave basin study was conducted in October 1992 to provide concurrent measurements of $\sigma, W_{c}$, and $k_{L}$ under controlled, reproducible conditions. The data from this experiment will be used to refine the empirical model of whitecap-mediated gas exchange developed with the WST data. A second wave-basin experiment is planned for October 1993.

Results to Date: LPDA bubble measurements of bubble populations produced in the WST have shown them to be very similar to occanic bubble populations. The LPDA turbulence and bubble measurements are being incorporated into available models for bubble driven gas exchange. The results of this effort are providing theoretical support and understanding for the empirical parameterization. The empirical parameterization developed from gas exchange measurements in the WST allows estimation of $k_{L}$ from $B_{c}, D$, and $\alpha$ for a wide range of gases, including $\mathrm{CO}_{2}$.

The analysis of the 1990 Georges Bank dual-tracer gasexchange experiment have shown that $k_{L}$ is linearly correlated with $W_{c}$ under oceanic conditions. The wavebasin results have shown that $k_{L}$ can be correlated with $\sigma$. The results from the Sequim Bay experiment have shown that sea-surface microwave emissivity can be correlated with parameters related to subsurface hubble dynamics.

\section{MEASUREMENTS OF $\mathrm{CO}_{2}$ IN SEAWATER ON WOCE HYIROGRAPHIC LINE P-17}

BINGLER, LINDA

\section{BATTELLE MARINE SCIENCES LABORATORY}

$\begin{array}{lr}\text { FY } 1993 & 66 \\ \text { FY } 1992 & 0 \\ \text { FY 1991 } & 0\end{array}$

$05 / .5 / 92-05 / 14 / 93$ 
Objective: To obtain accurate dissolved inorganic carbon measurements in water samples taken along WOCE lines in the Pacific Ocean.

Product: Tabulated inorganic carbon and hydrographic data and values for certified reference materials analyzed for quality control.

Approach: Sea-water samples will be drawn from Niskin-bottle profiles taken at WOCE hydrographic stations along lines in the Pacific Ocean. One 36-bottle cast will be analyzed per day at sea with an automated dissolved inorganic carbon analyzer. The $\mathrm{CO}_{2}$ analyses will be performed in accordance with the procedures in the $\mathrm{DOE} \mathrm{CO}_{2}$ Analysis Handbook. The data will be submitted to the $\mathrm{CO}_{2}$ Science Team Working Group on data quality for evaluation prior to submittal to the Carbon Dioxide Information and Analysis Center (CDIAC) for archiving and distribution.

Results to Date: The data from P-13 and P-14C were processed, checked, and submitted to CDIAC. Shipboard $\mathrm{CO}_{2}$ analyses were performed during line P-17 aboard the R/V Knorr. The data will be reduced and reported to CDIAC early in FY 1994.

\section{GEOCHEMICAL CONSTRAINTS ON OCEAN GENERAL CIRCULATION MODELS}

BROECKER, WALLACE S.

\section{LAMONT-DOHERTY EARTH OBSERVATORY}

$\begin{array}{lr}\text { FY } 1993 & 274 \\ \text { FY } 1992 & 0 \\ \text { FY } 1991 & 0\end{array}$

05/01/93-04/30/94

Objective: To compile an ocean chemical-tracer data set and verify/validate ocean-model calculations through comparison to the measured tracer data.

Product: A test of the model's ability to simulate the chemical and isotopic constituents of the ocean and an assessment of the data set.

Approach: The Maier-Reimer ocean GCM and two simpler models will be used in colored-water experiments. The models will be run to steady state at 10,30 , and 100 years with initial trace introduction. The distribution of the tracers and water properties will then be compared to measurements. The simpler models will be used in subsequent analyses to understand the comparisons. Such questions as the extent of cross-isopycnal mixing, the limits on subgrid-scale-process parameterizations (e.g., deep-water formation), and the validity of using observed values for some constituents (e.g., surface salinity) will be explored. Target tracers include natural radiocarbon, bomb tritium, bomb radiocarbon, freons, ${ }^{18} \mathrm{O},{ }^{13} \mathrm{C}$, phosphate, $\mathrm{CO}_{2}$, alkalinity, and silica.

The isotope ${ }^{14} \mathrm{C}$ provides an excellent tracer with natural and bomb-produced distributions for examination. Two approaches will be used to obtain ${ }^{14} \mathrm{C}$ data. First, radiocarbon data on $\mathrm{CaCO}_{3}$ will be obtained from archived mollusk shells collected alive from the mid- to high-latitude regions in the Southern Ocean prior to 1950. These data will provide the surface ocean distribution of radiocarbon prior to nuclear testing and can be used as an initial model input or can be compared with model calculations to test the model.

Ships of opportunity (research vessels) will be used to obtain water samples for analysis of radiocarbon and seawater constituents. Target cruises will be determined by analysis of the geographic distribution of the existing data. The data will be used to further the development of the tracer data as a tool for model validation and verification.

Results to Date: New project.

STEADY-STATE AND TRANSIENT MODELING OF TRACER AND NUTRIENT DISTRIBUTIONS IN THE GLOBAL OCEAN

BROECKER, WALLACE S.

\section{LAMONT-DOHERTY EARTH OBSERVATORY}

$\begin{array}{ll}\text { FY } 1993 & 129 \\ \text { FY } 1992 & 118 \\ \text { FY } 1991 & 129\end{array}$

08/01/93-07/31/94

Objective: To diagnosis the time-dependent relationship between ocean circulation and biological activity relative to the ocean carbon cycle.

Product: New information on and a tool to explore the transient behavior of the interactions among ocean circulation, biological activity, and the carbon cycle. 
Approach: A 2-D, zonally averaged ocean model will be extended to include ocean tracers and nutrients. Inclusion of the ocean tracers will assist in the validation of the model and the diagnosis necessary to improve the model capabilities. The transient behavior of the tracer distributions will be analyzed. This transient calculation includes the interaction of the ocean circulation and tracer/nutrients as greenhouse-gasinduced climate change progresses over paleoclimate time scales.

First, bomb-tritium data will be used to constrain the model's dynamics and parameterizations. To capture possible mode changes, the glacial-to-interglacial time scale will be investigated. Then the model will be used to study two scenarios for $\mathrm{CO}_{2}$-induced warming and to simulate the $\mathrm{CO}_{2}$ change at the termination of the last ice age. Further, the nutrient cycle will be incorporated into the model and validated. The completed model will then be used to identify the physical processes governing the ocean uptake of $\mathrm{CO}_{2}$. This information will be used to tune the nutrient/physical interactions within the model.

Dissolved-oxygen experiments will be used as a tïnal check on the model before conducting experiments and analyses to characterize how the dynamics of the ocean influence the nutrient cycle during climate change.

Results to Date: The balance of stable and decaying tracers was incorporated into a latitude-depth coupled ocean circulation model. The analysis of the tracer distributions enabled a census of the deep water masses. Modification of the surface buoyancy forcing brings the distributions into closer agreement with observed measurements and could also account for long-term, globalscale changes of the global thermohaline circulation.

Uptake of carbon was simulated by using an inorganiccarbon-cycle model and performing $2 \times \mathrm{CO}_{2}$ and anthropogenic perturbation. Experiments yielded an average uptake rate of about $2 \mathrm{GtC} / \mathrm{y}$ in the 1980s. The uptake of bomb radiocarbon indicates that the influence of the wind-driven circulation must be parameterized but does not greatly affect the net inventory changes.

The evolution of isotopes during rapid circulation changes was simulated and compared to the paleoclimatic record. The model is capable of qualitatively reproducing overshoots of ${ }^{18} \mathrm{O}$ and plateaux of radiocarbon age observed at the termination of the Younger Dryas.
An organic carbon cycle with sedimentation chemistry has now been included, and broad agreement with the observations has been achieved. The transient experiments will be repeated, allowing a first estimate of the magnitude of $\mathrm{pCO}_{2}$ changes during rapid changes of the global thermohaline circulation.

\section{OCEAN DATA}

CUSHMAN, ROBERT, and BODEN, THOMAS

\section{OAK RIDGE NATIONAL LABORATORY}

$\begin{array}{lr}\text { FY 1993 } & 350 \\ \text { FY 1992 } & 0 \\ \text { FY 1991 } & 0\end{array}$

$$
10 / 01 / 92-09 / 30 / 93
$$

Objective: To acquire, inspect, document, archive, and distribute DOE-sponsored ocean-carbon measuręments collected during or derived from the Joint Global Ocean Flux Study (JGOFS) aboard World Ocean Circulation Experiment (WOCE) cruises.

Product: Compiled or acquired numeric data packages (NDPs) consisting of written documentation and machine-readable files of ocean carbon data from the JGOFS program distributed free of charge via a variety of media, including 9-track magnetic tape, CD-ROM, IBM-formatted floppy diskettes, and an anonymous fïle transfer protocol (FTP) area over the Internet.

Approach: A data-management plan will be formulated to ensure submission of data to the Carbon Dioxide Information Analysis Center (CDIAC) and subsequent review, documentation, archiving, and distribution of DOE-sponsored oceanographic data from the JGOFS program. Data documentation prepared by CDIAC must be reviewed and approved by the contributing scientist(s) before distribution. CDIAC will attempt to coordinate its data management efforts with JGOFS and WOCE data-management efforts.

Results to Date: A data-management plan was compiled and presented to the DOE Oceanographic Science Team at its Seatte meeting in November 1992. A modified plan was presented and tentatively accepted at the Miami meeting in March 1993. The written version of the modified plan was reviewed by the DOE Science Team members and by the principal investigators at Lamont-Doherty Earth Observatory (LDEO). 
CDIAC and LDFO staff met on two occasions to begin coordination of future data-quality-assurance efforts and to discuss the roles and to assess the capabilities of each group. Other meetings were held to coordinate CDIAC's oceanographic data efforts with those of WOCE and to review the Data Management Plan with the JGOFS Steering Committee.

An NDP has been compiled describing carbon and hydrographic measurements collected during the 1990 WOCE 11/5 cruise in the South Atlantic Ocean, Weddell Sea, and Drake Passage. Carbon measurements were made by scientists at LDEO, and hydrographic measurements were made by researchers and technicians at the Oceanographic Data Facility at Scripps Institution of Oceanography. These data are still considered preliminary, and release of the NDP is contingent on the final release of the data by the German chicf scientist.

\section{DEVELOPMENT OF OCEANIC DEEP-CONVECTION MODELS FOR CLIMATE RESEARCH}

DENBO, D. W., and SKYLLINGSTAD, E. D.

\section{BATTELLE MARINE SCIENCES LABORATORY}

$\begin{array}{ll}\text { FY } 1993 & 223 \\ \text { FY } 1992 & 263 \\ \text { FY } 1991 & 234\end{array}$

$10 / 01 / 92-09 / 30 / 93$

Objective: To design and test a numerical model of open-water deep convection in the ocean and to apply the model to the development of improved parameterizations in future ocean models used for climate studies.

Product: A numerical model of deep oceanic convection, validated with laboratory and field data and including the important processes.

Approach: The 2-D upper-ocean model developed during the initial phase of this project will be validated by comparing nondimensional statistics, such as the wave-number spectra and heat flux, to laboratory data and atmospheric-mixed-layer data reported in the scientific literature. These comparisons will determine if the subgrid-scale turbulence model is properly adjusted and will test if the resolved eddy structure is realistic.
The 2-D model will be used to simulate deep convection with initial conditions from measurements in oceanic regions where convection occurs. The first of these simulations will use vertical temperature and salinity profiles taken in the Greenland Sea to analyze the structure and evolution of convective plumes in waters where the vertical structure of temperature and salinity support thermobaric effects. The model will be used to examine the increase in mixing caused by thermobaric plumes by comparing nondimensional vertical heat flux calculated with and without thermobaric effects. The model will also be applied to in the Medoc region of the Mediterranean Sea with initial conditions observed prior to the seasonal Mistral cold period and surface heat flux derived from observations. This simulation will run for approximately 20 simulation days. The model vertical profiles of temperature and salinity will be compared with measurements taken at the end of the 20-day cooling period to test the model's ability to simulate convection when thermobaric processes are not significant.

Initial testing of the 3-D model will use data from laboratory tank experiments of convection on a rotating plane. Further testing of the 3-D model will follow the same approach used for the 2-D model.

Results to Date: Construction and testing of the 3-D model is complete. The horizontal wave-number spectra of the velocity components and temperature from the 3-D model are in excellent agreement with laboratory measurements and theory for the inertia-convective subrange. Experiments to explore the effect of the upward and northward components of the Coriolis force on deep convection were conducted with smoothed temperature and salinity profiles taken in the Greenland Sea. For the nonrotating case, convective plumes travel straight downward, and horizontal motions are radial out from the plume center. With only the upward component of the Coriolis force present, the convective plumes travel straight downward with cyclonic horizontal motions.

With both components, the plumes slant to the east as they descend in addition to the cyclonic horizontal motions. For the experiments with the Coriolis forces, a horizontal cell structure exists for the vertical motions during plume formation. Penetrative plumes are formed at the vertices of these cells and are 300 to $400 \mathrm{~m}$ in diameter at a depth of about $900 \mathrm{~m}$ with a maximum vertical velocity of 5 to $10 \mathrm{~cm} / \mathrm{s}$. These scales are in very good agreement with Doppler-acoustic-velocity measurements made in the Greenland Sea. 


\section{EXPENDABLE BOTTOM-LAUNCHED CTD PROFILER}

DEROOS, BRADLEY G.

BATTELLE MEMORIAL INSTITUTE

$\begin{array}{lr}\text { FY } 1993 & 148 \\ \text { FY } 1992 & 150 \\ \text { FY } 1991 & 0\end{array}$

$10 / 01 / 92-09 / 30 / 93$

Objective: To develop an expendable continuous temperature-depth (CTD) profiler with satellite telemetry capabilities for long-term hydrographic observations in remote areas of the ocean.

Product: A prototype expendable CTD profiler with satellite data transmission and ground-positioning capabilities.

Approach: Existing methods for CTD profiling require costly surface support. Currently, there are no off-theshelf sensors well suited for making observations in remote regions. This research will develop a prototype sensor package for obtaining hydrographic data inexpensively. The sensor package will have microprocessor-controlled sampling and launch schedules, satellite telemetry, and ground-positioning capabilities and will operate as a surface drifter after profiling missions.

Results to Date: Successful testing of ceramic pressure housings, in several configurations, was completed. Tests to 8000 psia were made for a variety of ceramictube lengths and titanium-end-cap designs to determine the most reliable configuration for deep-sea use. It was demonstrated that (1) commercially available polyurethane adhesives perform very well at high pressure; (2) stock $6.4-\mathrm{cm}-\mathrm{ID}$ ceramic tubes with a $3.2-\mathrm{mm}$ wall and lengths up to $0.6 \mathrm{~m}$ also perform well, but preselection of round, straight lengths is essential; (3) grinding of the tube ends to flatten them extends the maximum operational depth of the ceramic housing significantly; and (4) a simple end seal of polyurethane between the flat ceramic tube end and the mating titanium cap is adequate for deep-sea pressures. A patent application was filed for the housing-construction technique. Other tests were performed with a fiber-wound design, but these were unsuccessful. Construction of a high-pressure test facility for oceanographic sensors was completed at the Battelle Marine Sciences Laboratory. Using this facility, long-term stability tests (about six months) of solid-state pressure transducers were initiated.

\section{QUALITY CONTROL OF OCEANIC CARBON DIOXIDE MEASUREMENTS: PREPARATION AND DISTRIBUTION OF REFERENCE MATERIALS \\ DICKSON, ANDREW G.}

SCRIPPS INSTITUTION OF OCEANOGRAPHY

$\begin{array}{lr}\text { FY 1993 } & 239 \\ \text { FY 1992 } & 237 \\ \text { FY } 1991 & 0\end{array}$

05/01/93-04/30/94

Objective: To develop and provide reference materials for $\mathrm{CO}_{2}$ in seawater; coordinate and develop standard measurement procedures; provide quality-control guidance for measurements; and develop an automated, ultraprecise $\mathrm{pH}$ method to monitor the long-term stability of reference materials.

Product: Reference materials (RMs) necessary to ensure that $\mathrm{CO}_{2}$ analyses made by several laboratories, working independently at sea, are consistent.

Approach: Reference materials for total dissolved inorganic carbon $\left(C_{T}\right)$ and alkalinity $\left(A_{T}\right)$ will be prepared and bottled. Four to six batches of $R M$ (depending on cruise schedules and at-sea requirements) will be produced, certified, and distributed each year of the project. Reference materials will be distributed to DOE survey participants as well as to investigators in the JGOFS time-series program and foreign laboratories working on international WOCE ships. The Scripps team will track and document the performance of the RMs.

An ongoing, collaborative study of $C_{T}$ analytical procedures will be supported, and similar studies for $A_{T}$, $\mathrm{pH}$, and $\mathrm{CO}_{2}$ fugacity $\left[\mathrm{f}\left(\mathrm{CO}_{2}\right)\right]$ will be initiated. Guidance will be provided on quality control of the global $\mathrm{CO}_{2}$ survey, and assistance will be provided with the cross checking of calibration standards among WOCE and JGOFS analysts by revising and maintaining the DOE Handbook of Methods and by distributing updated versions to DOE, JGOFS, and other investigators involved with oceanic $\mathrm{CO}_{2}$ measurements. 
An automated, ultraprecise $\mathrm{pH}$ method to monitor the long-term stability of reference materials distributed during the DOE survey will be developed and applied.

The data produced by this research will be incorporated in the ocean $\mathrm{CO}_{2}$ data package being assembled by the Carbon Dioxide Information and Analysis Center at ORNL.

Results to Date: Four batches of RMs have been prepared, certified, and distributed for use. These RMs have been used by scientists from $14 \mathrm{CO}_{2}$ groups, both in the United States and abroad, with the bulk of the materials being used directly to support the efforts of DOE-funded scientists.

A second collaborative study was conducted for the measurement of total dissolved inorganic curbon in seawater. Duplicate samples at five levels were sent to more than 20 laboratories around the world. Results have been received from 17 of these laboratories, and a report is currently in preparation.

The DOE Handbook of Methods has been extensively revised, and methods have been incorporated for the measurement of $\mathrm{pH}$ and of $\mathrm{f}\left(\mathrm{CO}_{2}\right)$. This version will be available for distribution after it has been approved by the Science Team.

\section{CARBON DIOXIDE OCEAN RESEARCH}

DOWNING, JOHN P.

\section{BATTELLE MARINE SCIENCES LABORATORY}

$\begin{array}{lr}\text { FY } 1993 & 83 \\ \text { FY } 1992 & 173 \\ \text { FY } 1991 & 438\end{array}$

$10 / 01 / 92-09 / 30 / 93$

Objective: To provide technical and scientific assistance to the Environmental Sciences Division (ESD) for coordination of the global survey of $\mathrm{CO}_{2}$ in the ocean (SRGP 92-8) and the Atmospheric Radiation Measurement Program - Ocean Measurements Working Group (OMWOG), Small Business Innovative Research (SBIR) on advanced sensing technologies for ocean measurements, process studies for improved ocean-circulation models for climate research, and production of an ocean-research bulletin.
Product: Technical reports, memoranda, and draft research plans to assist the ESD with program development, CEES and interagency coordination of ocean research, and science evaluations.

Approach: Ocean research will include process studies, data analysis, and ocean-model development designed to improve existing ocean simulation and prediction capabilities. These activities will be conducted in anticipation of large multiagency ocean observations to be conducted in the 1990s, such as WOCE and JGOFS. The results will provide a better framework to analyze the large data streams expected during the expected 5 to 10 years of these programs.

Results to Date: The $\mathrm{CO}_{2}$ Science Team met three times during the past year to oversee increasing shipboard activities for the global survey. The meetings were with the JGOFS Science Steering Group in Seattle in November, in Miami in March, and in Seattle in September. The September meeting included a workshop on coulometric analysis of $\mathrm{CO}_{2}$ in seawater for U.S. and international survey participants (conducted jointly with the NOAA Pacific Marine Environmental Laboratory).

The ARM Ocean Measurements Working Group met three times to assist with the design of a CART site in the tropical western Pacific Ocean. Chairmanship of the OMWOG was assumed in July 1993, and a working group was led for the international workshop Terrestrial Biospheric Carbon Fluxes: Quantification of Sinks and Sources of $\mathrm{CO}_{2}$.

In consort with the program managers of ESD, a new approach was developed to increase the effectiveness of ESD's SBIR program during the next 2 years. Two new commercial products were brought to market as a result of SBIR grants from the ocean-measurements topic. One is a pH measurement system for moored operations, and the other is a meter for measuring spectral absorption in seawater. 


\section{COULOMETER INTERCOMPARISON EXPERIMENT}

DOWNING, JOHN P.

\section{BATTELLE MARINE SCIENCES LABORATORY}

$\begin{array}{lr}\text { FY } 1993 & 215 \\ \text { FY } 1992 & 170 \\ \text { FY } 1991 & 30\end{array}$

$10 / 01 / 92-09 / 30 / 93$

Objective: To determine the consistency of dissolved inorganic carbon (DIC) analyses made by coulometers operated by the shipboard teams participating in the global survey of $\mathrm{CO}_{2}$ in the ocean.

Product: Prototype instrumentation and sampling systems.

Approach: The laboratory experiments, begun in prior years of the project, will be continued at Scripps Institution of Oceanography under separate funding. The standardization of coulometric $\mathrm{CO}_{2}$ analyzers and prototyping of titrators for alkalinity measurements in seawater will be the main project focus. We will work with the sea-going chemists to improve the SOMMA coulometric $\mathrm{CO}_{2}$ analyzers, update the SOMMA Operator's Manual, and develop a disposable, plasticmetal-plastic laminate seawater container for moreefficient water sampling aboard ship.

Results to Date: Four SOMMA $\mathrm{CO}_{2}$ analyzers werc built under contract by the University of Rhode Island and were added to the newly formed equipment pool for the global survey. The new SOMMAs were distributed to the University of Hawaii, University of Miami, Woods Hole Oceanographic Institution, and Princeton University. Several improvements were made to these instruments, including new software, new samplehandling procedures, spreadsheet outputs and calibration diagnostics, $\pm 0.01 \mathrm{C}$ temperature sensor calibration, color-coded gas service tubing, and increased durability achieved with unbreakable polyethylene base. The SOMMAs were furnished with electronic barometers and calibration systems. Version 2.0 of the Operators Manual was published and distributed. Prototype disposable seawater sample containers were fabricated and tested to evaluate the stability of carbonates in seawater stored in them for several minutes to several hundred hours. The development of two prototype, PC- controlled titrators for determining alkalinity in seawater was completed. The development of these titrators represents an expansion of the original scope of this project in response to a recommendation from the $\mathrm{CO}_{2}$ Science Team.

\section{MIXED-LAYER MODEL}

EMERSON, STEVEN

UNIVERSITY OF WASHINGTON

$\begin{array}{lr}\text { FY } 1993 & 50 \\ \text { FY } 1992 & 0 \\ \text { FY } 1991 & 72\end{array}$

08/01/93-07/31/94

Objective: To understand the utility of chemical tracers for determining biological fluxes in the ocean at Station $P$ and the JGOFS time-series station in the subtropical Pacific Ocean by using an ocean mixed-layer model incorporating chemical tracers and an ecosystem paradigm.

Product: An upper-ocean model for interpreting the time series of temperature, salinity, nutrients, ${ }^{14} \mathrm{C}$ primary productivity, chlorophyll, dissolved organic and inorganic carbon, and particle fluxes being acquired at the JGOFS station north of Hawaii.

Approach: A mixed-layer model of the Price-WellerPinkle type will be used to reproduce the density field observed at Station P in the subarctic North Pacific Ocean. To this model, an ecosystem structure, including mechanisms for nutrient cycling, will be added to relate mass fluxes of metabolic constituents to observable biological quantities. Gas-exchange rates at the air-water boundary of the model will be verified by reproducing measurements of the inert gases $\mathrm{N}_{2}, \mathrm{Ar}$, and ${ }^{222} \mathrm{Rn}$. With calculated nutrient fluxes and air-water gasexchange rates from the model, mixed-layer oxygen and perhaps $\mathrm{CO}_{2}$ concentrations are a potential measure of new biological production. Once the model performs well for Station $\mathrm{P}$, it will be applied to the emerging data from the JGOFS time-series station near Hawaii.

Results to Date: None reported. 


\section{DIAGNOSTIC ANALYSIS OF IRON LIMITATION OF $\mathrm{CO}_{2}$ UPTAKE BY OCEANIC PHYTOPLANKTON}

FALKOWSKI, PAUL G.

BRCOKHAVEN NATIONAL LABORATORY

$\begin{array}{lr}\text { FY } 1993 & 100 \\ \text { FY } 1992 & 82 \\ \text { FY } 1991 & 107\end{array}$

$10 / 01 / 91-09 / 30 / 92$

Objective: To develop a diagnostic technique to determine iron limitation of phytoplankton production.

Product: The ability to quantitatively assess any iron or other trace-metal nutrient limitation in subarctic and Antarctic surface ocean waters.

Approach: A variety of techniques will be used, including biophysical, biochemical, and molecular, to assess the effects of iron deficiency on phytoplankton. Controlled laboratory studies that use iron-limiting cultures will provide basic information on the response of the photosynthetic apparatus to iron limitation. Intraspecific comparisons will be made between phytoplankton from several taxonomic groups. Field studies with natural phytoplankton populations in Southern Ocean will be conducted to determine whether natural iron concentrations limit phytoplankton biomass and productivity.

Results to Date: The FeLine cruise in the equatorial Pacific was participated in to test the hypothesis that ocean-phytoplankton photosynthesis is iron limited in this region of the world. The equatorial Pacific is estimated to support ca. $20 \%$ of the global new primary production (i.e., the fraction of oceanic carbon fixation that, in the steady-state, is exported to the ocean interior). The new fast repetition rate fluorometer (FRR) developed at Brookhaven National Laboratory (BNL) was used to measure changes in the maximum quantum efficiency and absorption cross sections of the photosynthetic apparatus of phytoplankton in situ along a section extending from the Equator to $15^{\circ} \mathrm{N}$ at $140^{\circ}$ $W$. In the iron-limited regions, maximum quantum yields were reduced by $50 \%$ relative to nutrient-replete regions. The quantum yield could be enhanced within $36 \mathrm{~h}$ following the addition of nanomolar quantities of soluble iron or by adding extracts of aerosol particles.
North of $6^{\circ} \mathrm{N}$ latitude, surface nitrate values were at or below the level of detection, and iron had no effect on quantum efficiency. Samples of specific proteins were obtained for analysis with polyacrylamide-gelelectrophoresis and western-blotting techniques. The protein analyses will be used to look for the presence of key proteins indicative of iron stress, for which we have antibodies. Additional fluorescence lifetime measurements under iron-replete and -deficient conditions establish that iron limitation results in impairment of photosystem II charge separation. This capability is restored within $24 \mathrm{~h}$ following addition of iron, and molecular and biophysical analyses suggest that two proteins, CP47 and cytochrome b559, are depleted under iron limitation. Overall, our data strongly suggest that, in the equatorial Pacific, iron limits phytoplankton carbon fixation at a molecular level by impairing photosynthetic energy-conversion efficiency within photosystem II. As such, these data refute any hypothesis that in this region phytoplankton production is regulated by grazing or irradiance.

\section{$\mathrm{CO}_{2}$ MEASUREMENTS ALONG WOCE SECTIONS IN THE PACIFIC AND INDIAN OCEANS}

\section{GOYET, CATHERINE}

\section{WOODS HOLE OCEANOGRAPHIC INSTITUTION}

$\begin{array}{lr}\text { FY } 1993 & 210 \\ \text { FY } 1992 & 0 \\ \text { FY } 1991 & 0\end{array}$

$$
01 / 01 / 93-12 / 31 / 93
$$

Objective: To contribute accurate data on the carbon chemistry of seawater to a global oceanic database for numerical modeling,

Product: A data report of tabulated carbon-chemistry properties of Pacific and Indian Ocean seawaters.

Approach: Three carbon-chemistry properties will be measured in seawater along WOCE cruises in the Pacific and Indian oceans. Total $\mathrm{CO}_{2}$ and total alkalinity will be measured throughout the water column from approximately one-third of the WOCE stations (approximately 40 samples per day). The partial pressure of $\mathrm{CO}_{2}$ in surface seawater will be continually monitored along the cruise tracks. Aboard ship, total $\mathrm{CO}_{2}$ will be measured by using a coulometer, and total 
alkalinity analyses will be performed with an automated potentiometric system. $\mathrm{CO}_{2}$ partial pressure will be determined with a shower-head equilibrator and an infrared analyzer.

Results to Date: During the WOCE cruise P-17N (May 15 to June 26, 1993), 1112 samples were collected for total $\mathrm{CO}_{2}$ and total alkalinity measurements; 32 stations were sampled throughout the water column, and 51 stations were sampled in the upper $200 \mathrm{~m}$. In addition, 40 samples were collected at 10 stations for C. D. Keeling. $\mathrm{CO}_{2}$ partial pressure in surface seawater has been monitored along the cruise track from San Francisco to Sitka.

OCEAN $\mathrm{CO}_{2}$ MEASUREMENTS FOR THE WOCE HYDROGRAPHIC SURVEY IN THE

PACIFIC OCEAN, 1992-1993: SHORE-BASED ANALYSES OF DISSOLVED INORGANIC CARBON

KEELING, CHARLES D.

SCRIPPS INSTITUTION OF OCEANOGRAPHY

$\begin{array}{lr}\text { FY } 1993 & 205 \\ \text { FY } 1992 & 0 \\ \text { FY } 1991 & 0\end{array}$

$01 / 01 / 93-12 / 31 / 93$

Objective: To provide shore-based replicate analysis of the $\mathrm{CO}_{2}$ data obtained by the at-sea global ocean $\mathrm{CO}_{2}$ survey.

Product: Verification of the at-sea measurements from the global survey.

Approach: It is critical in a survey conducted over several years and by multiple laboratories with changing personnel that data be consistent not only during the survey but also in relationship to prior work. This effort will provide a consistency check. The standards and methods will tie the nearly four-decade atmospheric record to ocean surveys conducted in the early seventies and eighties with the first phase of the DOE program. This will be accomplished by replicate analysis of water samples preserved at sea during WOCE cruises with the precision, manometric total $\mathrm{CO}_{2}$ analyzer. Replicate analyses will be performed on approximately 500 preserved water samples from WOCE lines in the Pacific Ocean (P-6, P-13, P-14, P-16, P-17, and P-19), samples from the Atlantic Ocean (A-10), and 100 control samples for the certification of seawater reference materials.

Results to Date: The concentration of dissolved inorganic carbon in seawater has been determined by gas extraction followed by manometric assay on a series of water samples collected as part of the WOCE Hydrographic Survey of the Pacific Ocean. The samples were collected on cruises in which the $\mathrm{DOE} \mathrm{CO}_{2}$ Science Team participated in at-sea measurements of dissolved inorganic carbon. They pmvide a check on the performance of coulometric analyzers used at sea and how well these analyzers are being calibrated with standard reference seawater. The ${ }^{13} \mathrm{C} /{ }^{12} \mathrm{C}$ ratio of dissolved inorganic carbon has been determined to provide isotopic data beyond the primary objective of documenting the levels of inorganic carbon in the waters of the Pacific Ocean. The combined data will help establish the rate of uptake on industrial $\mathrm{CO}_{2}$ by the oceans and the extent to which this uptake mitigates the buildup of $\mathrm{CO}_{2}$ in the atmosphere.

\section{OCEANIC $\mathrm{CO}_{2}$ MEASUREMENTS FOR THE WOCE HYDROGRAPHIC SURVEY OF THE \\ PACIFIC OCEAN: SHIPBOARD ALKALINITY ANALYSES DURING 1991 AND 1992}

KEELING, CHARLES D.

\section{SCRIPPS INSTITUTION OF OCEANOGRAPHY}

$\begin{array}{lr}\text { FY } 1993 & 39 \\ \text { FY } 1992 & 150 \\ \text { FY } 1991 & 97\end{array}$

$02 / 01 / 93-01 / 31 / 94$

Objective: To conduct shipboard measurements of titration alkalinity on seawater samples collected along WOCE/HP lines in the equatorial and southern Pacific Ocean and to use the resulting data in conjunction with $\mathrm{pCO}_{2}$, dissolved inorganic carbon (DIC), and shorebased alkalinity measurements.

Product: Internal consistency and reliability of oceanic $\mathrm{CO}_{2}$ data.

Approach: Titration alkalinity (ALK) of seawater samples obtained along WOCE lines in the Pacific Ocean will be determined at sea by potentiometric acid titration. The ALK titrations will be made in conjunc- 
tion with measurements of DIC by other DOE-supported shipboard teams. The ALK data will be compared with measurements of $\mathrm{CO}_{2}$ partial pressure made by equilibrator/gas chromatography at sea and with shore-based DIC and ALK measurements on replicate water samples. The combined data set will provide a critical test of the thermodynamic equations used to connect $\mathrm{PCO}_{2}$, DIC, and ALK.

\section{U.S. WOCE PROJECT OFFICE}

LAMBERT, R., and NOWLIN, W. D.

NATIONAL SCIENCE FOUNDATION

$\begin{array}{lr}\text { FY } 1993 & 25 \\ \text { FY } 1992 & 25 \\ \text { FY } 1991 & 0\end{array}$

$$
\text { 04/01/93-03/31/94 }
$$

Objective: To continue the World Ocean Circulation Experiment (WOCE) Project Office at Texas A\&M; to initiate a technical support office for interagency WOCE activities in Washington, D.C.; and to continue the U.S. support for the WOCE international office.

Product: Technical support for the interagency WOCE ad hoc panel.

Approach: Texas A\&M University will provide technical support for the planning and the implementation of the WOCE. This task will include developing scientific plans and maintaining scientific steering committees on instrumentation, standards, modeling, data management, and observational programs. As the WOCE moves from the planning to the operational stage, this activity will continue but at a reduced level.

Support will be provided in Washington, D.C., for the interagency team responsible for WOCE research. The office will provide meeting facilitation, manage oversight databases, and assist the National Science Foundation (NSF) as the lead agency for WOCE.

The U.S. WOCE science program will be supported and represented before the International WOCE Program Office, which serves much as the U.S. Project Office does as a clearinghouse for the science.

\section{THE CARBON DIOXIDE SYSTEM IN THE OCEAN}

\author{
MILLERO, FRANK J.
}

UNIVERSITY OF MIAMI

$\begin{array}{lr}\text { FY } 1993 & 233 \\ \text { FY } 1992 & 0 \\ \text { FY } 1991 & 0\end{array}$

01/01/93-12/31/93

Objective: To obtain total- $\mathrm{CO}_{2}$-concentration measurements at sea in the Atlantic and Pacific oceans and to measure alkalinity and measure $\mathrm{pCO}_{2}$ in the laboratory from samples brought to shore.

Product: Improved understanding of the ocean carbon cycle and a global synoptic view of inorganic carbon concentrations and transport in the world oceans for the development of advanced carbon-cycle models.

Approach: The chemists, instrumentation, and general support to perform $\mathrm{CO}_{2}$ system analyses at sea for up to 45 days per year will be provided. In the laboratory, total $\mathrm{CO}_{2}$ and $\mathrm{pCO}_{2}$ will be measured as primary parameters, and alkalinity will be measured as time permits. Further, pH will be measured to provide redundant determination of the system and to improve the thermodynamic model of carbonic acid in seawater. The effort will provide trained chemists and equipment, when available, to other sea-going groups. WOCE line $\mathrm{P}-14 \mathrm{Nb}$ will be covered.

Results to Date: New project.

\section{OCEANIC UPTAKE OF CARBON DIOXIDE}

PENG, T.-H

\section{OAK RIDGE NATIONAL LABORATORY}

$\begin{array}{ll}\text { FY } 1993 & 107 \\ \text { FY } 1992 & 110 \\ \text { FY } 1991 & 107\end{array}$

$10 / 01 / 92-09 / 30 / 93$ 
Objective: To improve the quantitative understanding of atmosphere-ocean $\mathrm{CO}_{2}$ interactions.

Product: An estimate of the potential for the world oceans to assimilate increases in atmospheric $\mathrm{CO}_{2}$ during the next century.

Approach: In cooperation with Lamont-Doherty Earth Observatory (LDEO), an iron-fertilized Antarctic Ocean will be simulated with dynamic considerations based on the distribution of bomb-produced ${ }^{14} \mathrm{C}$.

To improve upon the previous estimates of the $\mathrm{pCO}_{2}$ distribution in the world's oceans, it may be possible to exploit relations between the spatial distribution of $\mathrm{pCO}_{2}$ and existing nutrient-concentration data, which are routinely measured during most oceanographic expeditions. One likely such surrogate is the oceanic surface $\mathrm{PO}_{4}$ concentration. Thus, regional relationships will be established between $\mathrm{pCO}_{2}$ and $\mathrm{PO}_{4}$ for the world oceans in collaboration with LDEO, where most of the world ocean $\mathrm{pCO}_{2}$ data are stored. Winter and summer maps of the global distributions of delta $\mathrm{pCO}_{2}$ will be constructed and used to refine the calculations of net $\mathrm{CO}_{2}$ uptake by the world oceans.

Results to Date: During the past decade, atmospheric $\mathrm{pCO}_{2}$ at high latitudes in the Northern Hemisphere was, on the average, $3 \mu \mathrm{atm}$ higher than that over the high latitudes in the Southern Hemisphere. This gradient was much smaller a few decades ago, so a reverse atmospheric $\mathrm{CO}_{2}$ gradient may have existed prior to the industrial revolution. The release of fossil-fuel $\mathrm{CO}_{2}$ into the Northern Hemisphere by the industrialized countries slowly balances such natural south-to-north gradients; eventually, the gradient begins to tilt to the other direction. Keeling and Heimann proposed a south-tonorth interhemispheric transport of about 1 gigaton of carbon through the atmosphere in preindustrial time. This northward transport of carbon would have to have been compensated for by a southward transport of carbon through the ocean. We have attempted to evaluate this proposed mechanism by analyzing geochemical data taken as part of the Geochemical Ocean Sections Study (GEOSECS), Transient Tracers in the Ocean (TTO), and South Atlantic Ventilation Experiment (SAVE) programs. We concluded that the ocean did tend to transport $\mathrm{CO}_{2}$ from the Northern Hemisphere to the Southern Hemisphere in the preindustrial time. With the difference of $80 \mu \mathrm{mol} \mathrm{kg}{ }^{-1}$ in $\Delta \Sigma \mathrm{CO}_{2}$ between southwardflowing North Allantic deep water (NADW) and the northward-flowing return waters, the transport in the ocean of 0.6 gigaton of carbon per year from the Northern Hemisphere to the Southern Hemisphere could be possible in the preindustrial era. To balance this interhemispheric carbon flux in the ocean would require a south-to-north atmospheric $\mathrm{CO}_{2}$ gradient of approximately $1.2 \mu \mathrm{atm}$.

\section{GLOBAL SURVEY OF CARBON DIOXIDE IN THE OCEAN}

SARMIENTO, J. L., and KEY, R. M.

PRINCETON UNIVERSITY

$\begin{array}{lr}\text { FY } 1993 & 200 \\ \text { FY } 1992 & 0 \\ \text { FY } 1991 & 0\end{array}$

$01 / 01 / 93-12 / 31 / 93$

Objective: To obtain total- $-\mathrm{CO}_{2}$-concentration and $\mathrm{CO}_{2}$ partial-pressure $\left(\mathrm{pCO}_{2}\right)$ measurements in the South Pacific Ocean and the Pacific sector of the Southern Ocean; to measure alkalinity; and to measure $\mathrm{pCO}_{2}$ in surface waters and the atmosphere with a continuous equilibrator and infrared analyzer.

Product: An improved understanding of the ocean carbon cycle and a global synoptic view of inorganic carbon concentrations and transport in the world oceans for the development of advanced carbon-cycle models.

Approach: Total- $\mathrm{CO}_{2}$ measurements will be made in full accordance with accepted DOE procedures for the $\mathrm{CO}_{2}$ survey program with a commercial coulometer and an automated sample-stripping system. A circulating bath will be added to maintain temperature stability in the instrumentation system and to achieve the best possible accuracy.

The standard titration method, which will achieve $0.1 \%$ precision, will be used to analyze for alkalinity: The calibration of the $\mathrm{pCO}_{2}$ system will be addressed via the intercomparison experiments being conducted by the $\mathrm{CO}_{2}$ Science Team.

$\mathrm{CO}_{2}$ partial pressures and titration alkalinity will be measured along WOCE lines P-10, P-165, and P-175.

Results to Date: New project. 
OCEAN-SURFACE-LAYER DYNAMICS

SKYLLINGSTAD, E., and DENBO, D.

\section{BATTELLE MARINE SCIENCES LABORATORY}

$\begin{array}{ll}\text { FY } 1993 & 290 \\ \text { FY } 1992 & 251 \\ \text { FY } 1991 & 188\end{array}$

$10 / 01 / 92-09 / 30 / 93$ field of these structures to plots of measured oceanbubble plumes shows remarkable similarity.

Preliminary simulations have also been completed to examine the effects of strong shear on convection. These experiments show that the relative strength of the shear and cooling rate determine the behavior of circulation within the mixed layer. For example, with strong cooling and weak shear, circulations form in longitudinal rolls, much like Langmuir cells. However, with strong shear and weak cooling, transverse rolls are forced. These results are in agreement with theory that predicts the two circulation types.

\section{MEASUREMENTS OF CARBON DIOXIDE IN THE SOUTHERN OCEAN ALONG THE WOCE S-4 SECTION}

Objectlve: To determine, by numerical experiments on surface-layer dynamics, if a more detailed treatment of surface-layer processes will substantively change climate and atmospheric $\mathrm{CO}_{2}$ projections based on simple mixed-layer models.

Product: A numerical model of the turbulent dynamics of the surface mixed layer that will integrate existing field measurements and provide insight into the role of quantities that cannot be directly measured.

Approach: A 3-D model of the ocean surface layer will be used to examine processes important for mixing heat, momentum, and trace gases from the atmosphere to the intermediate waters below the thermocline. Processes to be examined include convection from nighttime conling, wind-wave-driven circulations, and convective-shear instabilities. Observations of mixed-layer dynamics, such as acoustic sonar measurements, will be used along with analytical results to verify the model accuracy. Simulation results will be applied to the development of improved parameterizations of mixed-layer processes, such as surface-wave-induced Langmuir circulations or internal waves in the thermocline.

Results to Date: The 3-D model developed earlier in this project was modified to include a parameterization of surface-wave interaction with a Stokes drift-vortex force. Preliminary results from the model with the Stokes drift parameterization as forcing show a consistent convective behavior for wind-forced circulations. When the model was started from rest with an applied wind stress and wave-vortex force, longitudinal roll structures formed with a wavelength approximately 3 times the initial mixed-layer depth. After 2 to $4 \mathrm{~h}$, these cells divided into complex structures, but retained a strong alignment with the wind and wave forcing. Comparison of the vertical-velocity
TAKAHASHI, TARO

\section{L.AMONT-DOHERTY EARTH OBSERVATORY}

$\begin{array}{lr}\text { FY } 1993 & 111 \\ \text { FY } 1992 & 169 \\ \text { FY } 1991 & 0\end{array}$

$03 / 01 / 93-02 / 28 / 94$

Objectlve: To determine accurately the carbon chemistry of seawater and to contribute to a global oceanic database for numerical modeling.

Product: Tabulated total $\mathrm{CO}_{2}$ concentrations and $\mathrm{CO}_{2}$ partial pressure in seawater from the Pacific sector of the Southern Ocean.

Approach: The total $\mathrm{CO}_{2}$ concentration and $\mathrm{CO}_{2}$ partial pressure in seawater will be measured in seawater from the Pacific sector of the Southern Ocean along the WOCE S-4 lines, $67^{\circ} \mathrm{S}$. Measurements of total $\mathrm{CO}_{2}$ and partial pressure of $\mathrm{CO}_{2}$ from about one-third of the 110 sampling stations will yield depth profiles of carbon chemistry in the Pacific Ocean. At least two samples of the surface mixed layer will be analyzed at every station. Aboard ship, total $\mathrm{CO}_{2}$ will be measured with a coulometer, and $\mathrm{CO}_{2}$ partial pressure will be determined with an equilibrator-gas chromatograph system.

Results to Date: From February 16, 1992, through April 6, 1992, the $\mathrm{CO}_{2}$ distribution along the WOCE S-4 section $\left(67^{\circ} \mathrm{S}\right)$ was investigated in the Pacific 
sector of the Southern Ocean between $73^{\circ} \mathrm{W}$ and $172^{\circ}$ E. Total $\mathrm{CO}_{2}$ concentration and $\mathrm{pCO}_{2}$ were determined in 1290 water samples collected at the 112 hydrographic stations, and 161 total $\mathrm{CO}_{2}$ determinations were made at sea for the Scripps Institution of Oceanography (SIO) $\mathrm{CO}_{2}$ reference solutions, yielding $1927.46 \pm 1.77$ $\mu \mathrm{mol} / \mathrm{kg}$. This value is in close agreement with the results of manometric measurements of $1925.74 \pm 0.74$ $\mu \mathrm{mol} / \mathrm{kg}$ (nine measurements) made by C. D. Keeling in his shore-based laboratory.

Along the $67^{\circ} \mathrm{S}$ parallel, the surface water had temperatures of about $1.0 \pm 0.2^{\circ} \mathrm{C}$ and $\mathrm{pCO}_{2}$ values about $30 \mu \mathrm{atm}$ below the atmospheric on the average. Hence, the ocean was a strong sink for atmospheric $\mathrm{CO}_{2}$ with an exception of two narrow $\mathrm{CO}_{2}$ source zones, which may be attributed to the local upwelling of deep waters rich in $\mathrm{CO}_{2}$. The total- $\mathrm{CO}_{2}$-concentration values in deep waters were nearly uniform below about $500 \mathrm{~m}$, ranging between 2250 and $2265 \mu \mathrm{mol} / \mathrm{kg}$. Low concentrations were found in the deep and bottom waters near $160^{\circ} \mathrm{W}$. This result may represent an affect of the Ross Sea water.

\section{MEASUREMENTS OF TOTAL $\mathrm{CO}_{2}$ CONCENTRATION, PARTIAL PRESSURE OF $\mathrm{CO}_{2}$, AND ALKALINITY IN SEAWATER DURING WOCE EXPEDITIONS IN THE SOUTH PACIFIC OCEAN}

TAKAHASHI, TARO

\section{LAMONT-DOHERTY EARTH OBSERVATORY}

$$
\begin{array}{lr}
\text { FY } 1993 & 456 \\
\text { FY } 1992 & 0 \\
\text { FY } 1991 & 0 \\
& \\
01 / 01 / 93-12 / 31 / 93
\end{array}
$$

Objective: To obtain total- $\mathrm{CO}_{2}$-concentration and $\mathrm{CO}_{2}$ partial-pressure $\left(\mathrm{pCO}_{2}\right)$ measurements in the South Pacific Ocean and the Pacific sector of the Southern Ocean and to investigate the effects of temperature on $\mathrm{CO}_{2}$ partial pressure to remove the uncertainty about temperature effects in calculated values for deep samples.

Product: The improved understanding of the ocean carbon cycle and a global synoptic view of inorganic carbon concentrations and transport in the world oceans for the development of advanced cartion cycle models.

Approach: The shipboard $\mathrm{CO}_{2}$ measurements will be made with the DOE-developed, automated coulometer system and by gas chromatography methods. The gas chromatographic measurements will be callbrated with $\mathrm{CO}_{2}$-air mixtures referenced to standards prepared by Scripps Institution of Oceanography (SIO) and the World Metcorological Offlce (WMO). About 7000 water samples will be analyzed at sea for total $\mathrm{CO}_{2}$ and $\mathrm{CO}_{2}$ partial pressure $\left(\mathrm{pCO}_{2}\right.$ ), and $10 \%$ (about 700 samples) will be preserved and analyzed for titration alkalinity at Lamont-Doherty.

WOCE lines P-165, P-175, and P-19 will be covered.

Results to Date: New project.

\section{INORGANIC CARBON MEASUREMENTS FOR THE WORLD OCEAN CIRCULATION EXPERIMENT - HYDROGRAPHIC PROGRAM}

WALLACE, D. W. R., and JOHNSON, $K$.

\author{
BROOKHAVEN NATIONAL LABORATORY

$\begin{array}{ll}\text { FY } 1993 & 468 \\ \text { FY 1992 } & 442 \\ \text { FY 1991 } & 283\end{array}$ \\ $10 / 01 / 91-09 / 30 / 92$
}

Objective: To develop instrumentation for and to collect comprehensive, intercomparable, high-quality $\mathrm{CO}_{2}$ data on samples collected during the World Ocean Circulation Experiment Hydrographic Program (WOCE/HP) one-time survey in the Allantic Ocean.

Product: Inorganic carbon and basic biomass data sets in sufficient density to allow calculation of carbon fluxes and storage with water-mass-transport and oceancirculation models; improved and more robust instrumentation that can be used by other survey participants.

Approach: Shipboard analysts on WOCE/HP cruises will analyze almost all water samples for dissolved inorganic carbon (DIC), $\mathrm{pH}$, and $\mathrm{pCO}_{2}$. DIC will be measured by automated coulometric titration (CT), $\mathrm{pCO}_{2}$ by headspace catalytic gas chromatography with 
flame-Ionization detection, and $\mathrm{pH}$ by a specially designed $\mathrm{pH}$ cell interfaced to the coulometric system. In addluion to inorganic $\mathrm{CO}_{2}$ measurements, the basic blomass parameters chlorophyll $a$, particulate organic carbon and nitrogen, and depth of the euphotic zone will be measured during selected cruises. These field measurements will allow carbon cycling to be related to remotely-sensed variables, such as chlorophyll.

Results to Date: Section A-10 was measured from Rio de Janeiro to Capetown, South Africa, from December to February 1993 aboard the FS Meteor. Data reports, which encompass more than 6000 analyses of CT made during more than 180 days at sea, are being prepared for submission to the Carbon Dloxide Information Analysis Center. The discrete $\mathrm{pCO}_{2}$ method was further tested aboard the R/V Gyre in May of 1993 and will undergo a final sea trial in July-August of 1993. Preliminary meridional-carbon-flux calculations have been carried outh and a third expedition aboard the Meteor in 1994 and WOCE Section A-15 aboard the Knorr are in the advanced stages of planning. The former cruise, when completed, will give us complete CT sections from the East Const of South America to the West Coast of Africa along the 10,20, and $30^{\circ} \mathrm{S}$ parallels. An additional four DOE-SOMMA systems have been built, certified, and distributed during 1993. Several additional systems are under construction. Final testing of a SOMMA-compatible pH measurement will be made in the fall of 1993.

\section{MEASUREMENTS OF SURFACE-OCEAN CARBON DIOXIDE PARTIAL PRESSURE DURING WOCE}

WEISS, R. F.

\section{SCRIPPS INSTITUTION OF OCEANOGRAPHY}

$\begin{array}{ll}\text { FY } 1993 & 95 \\ \text { FY } 1992 & 51 \\ \text { FY } 1991 & 48\end{array}$

$$
06 / 01 / 93-05 / 31 / 94
$$

Objective: To install, maintain, and operate automated analyzers aboard WOCE ships to measure $\mathrm{pCO}_{2}$ and $\mathrm{pN}_{2} \mathrm{O}$ in the atmosphere and surface waters of the Pacific, Allantic, and Indian oceans.

Product: The improved understanding of the ocean carbon cycle and high-temporal- and high-spatial- resolution surface data for Interpolation between discrete-sample values obtained by other ocean $\mathrm{CO}_{2}$ survey program participants.

Approach: Shipboard measurements of $\mathrm{pCO}_{2}$ and $\mathrm{pN}_{2} \mathrm{O}$ in surface waters and in the atmosphere will be inade along approximately three WOCE Hydrographic Pro. gram expedition legs per year. The measurements will be made with an automated, high-precision, gas-chromatographle system. $\mathrm{CO}_{2}$ will be measured with a flame-ionization detector after quantitative reduction to methane with palladium and nickel dual catalysts, and $\mathrm{N}_{2} \mathrm{O}$ will be measured with a separate electron-capture detector. Surface waters will be measured by equilibrating the measured gas phase with continuously pumped seawater with a two-stage equilibration chamber. The system will operate continuously, measuring the surface ocean and the atmosphere, each twice per hour.

Results to Date: The $\mathrm{pCO}_{2}$ and $\mathrm{pN}_{2} \mathrm{O}$ in surface waters and in the atmosphere were measured on three major WOCE legs in the Paclfic. These expedition legs (Knurr 138-9, 138-10, and 138-12) covered 152 days at sea and more than $36,000 \mathrm{~km}$, including the southern portions of WOCE lines P.16 and P.17 and all of WOCE line P.19. These lines are of special importance because they cover large areas of the southeastem Pacific that have not been previously sampled for these gases.

The southern portions of the three lines show both spatial and temporal variations in the distribution of surface ocean $\mathrm{pCO}_{2}$, On Knorr $138-9$ (October-November 1992), $\mathrm{pCO}_{2}$ is undersaturated north of about $50^{\circ} \mathrm{S}$ and oversaturated in the circumpolar waters to the south. As the season moves from spring into summer, the Knorr 138-10 values (December 1992-January 1993) show less undersaturation at low latitudes and shift from oversaturation to undersaturation at high latitudes. While a proper interpretation must depend on an analysis of these data together with the hydrographic and nutrient data, the observed shifts appear consistent with expected seasonal changes in biological productivity and, at lower latitudes, in water temperature. Corresponding variations in $\mathrm{pN}_{2} \mathrm{O}$ are less pronounced, with general oversaturathon everywhere, ranging from 1 or $2 \%$ at about $20^{\circ} \mathrm{S}$ to about $6 \%$ south of about $50^{\circ} \mathrm{S}$.

Along WOCE line P-19 (Knorr 138-12) at about $90^{\circ} \mathrm{W}$, both $\mathrm{pCO}_{2}$ and $\mathrm{pN}_{2} \mathrm{O}$ show the effects of upwelling along the eastern boundary of the Pacific. $\mathrm{CO}_{2}$ is markedly oversaturated north of about $45^{\circ} \mathrm{S}$, but the greatest oversaturations $(8010100 \mathrm{ppm})$ occur at the 
equator and at the northern end of the track, off the coast of Central America. $\mathrm{N}_{2} \mathrm{O}$ shows only about 5\% oversaturation along most of the track, but a very large peak (almost double the atmospheric value) is centered exactly on the Bquator, and lesser maxima occur near the Central American coust. Excesses in both of these gases are brought to the surface by upwelling, but the residence time with respect to loss to the atmosphere is much longer for $\mathrm{CO}_{2}$. Hence, the $\mathrm{N}_{2} \mathrm{O}$ signal is not as sensiluve to excesses advected westward from South America, but at the Equator, where vertical mixing is driven by the narrow Equatorial Undercurrent, the $\mathrm{N}_{2} \mathrm{O}$ sigral is affected more sharply.

\section{MEASUREMENT OF DIC, ALKALINITY, AND PH ON THE WOCE/HP CRUISES}

WINN, C. D., and MackENZIE, F. T.

\section{UNIVERSITY OF HAWAII}

FY 1993

FY 1992

193

FY 1991

$01 / 01 / 93-12 / 31 / 93$
Objectlvet To measure total $\mathrm{CO}_{2}$ concentration with coulometry, alkalinity with titration, and $\mathrm{pH}$ with spectrophometric techniques aloard WOCE vessels in the Pacific and Indlan oceans.

Product: An improved understanding of the ocean carbon cycle and a global synoptic view of inorganic. carbon concentrations and transport in the oceans of the world to allow developenent of advaneed carbon-cycle models.

Approuch Total $\mathrm{CO}_{2}$ will be measured with coulomctry, alkalinity with titration, and $\mathrm{pH}$ with spectrophometrle techniques. These measurements will be made aboard WOCE vassels in the Pucifle and Indian Oceans about 90 days per year. The data will be quallty controlled in accordance with DOE stundard operating procedures and procedures used for the Joint Olobal Occan Flux Study (JOOFS) Umes-scries station off the Hawall tslands. The research team will participate in the ongoing $\mathrm{CO}_{2}$ analysis intercompurison that is heing conducted to detect and quandfy systematic differences among $\mathrm{CO}_{2}$ data from analysts in the $\mathrm{CO}_{2}$ program. WOCE lines P.14Na and P.2 will be covered.

Resules to Datei New project. 


\section{ECONOMICS OF GLOBAL CLIMATE CHANGE}

The Economies of Clobal Change Program emphasizes rescarch that supports future decisionmaking; that is, rescarch on subjects that will form the eritical ceconomic unalysis for decisions expected to be made in the intermediate (2 to 5 years from now) and longer timeframes. It also emphusizes integrated analysis and the need for an economic framework that provides estimales of both benefits and costs of potential global change and actions to ameliorate it. The Program contains five main culegories: fundamental processes, integration, sectoral studies, intemational issues, and ussessments. In FY 1993 it highlighted two specific topics, (1) technology innovation and diffusion and (2) greenhouse-gas indices.

Technical Innovation and Diffusion: One of the most uncertain aspects in an integrated assussment is predicting the changes in future technology that would impact both the ability to mitigate greenhouse-gas emissions and to adapt to potential climale changes. The research program will determine appropriate rates of innovation for the different activities that would be modeled in an integrated assessment. For instance, the rute of technology innovation and diffusion could be different for technology that (1) improves the efficiency of energy use per unit output or per gross domestic producl (ODP), (2) decreases the umount of greenhouse gus per unit of energy use, or (3) helps sociely adapt to specific changes in the globul climate. Rescurch will help resolve whether assumptions that are made, for instance, regarding technology improvements in energy production should be correlated with equivalent assumptions, such us labor productivity, elsewhere in an integrated assessment. Even if no policy actions were taken by the United States, certain technology clunges would take place. The research program will help determine background levels of technology change as well as the differential impact of different policy instruments, such as carbon taxes or funding for additional research and development.

The research program also will help resolve the ability and willingness of sxcicty to absorb existing technology. This research would address an important controversy regarding the extent to which a significant reduction of greenhouse-gas emissions can be achieved with a net gain to ODP or at least very little cost. Some argue that most of the potential gain has already been accomplished and that further reductions imply significant costs to society. A resolution of this problem by comparing these two views (commonly known as top/ down and bottom/up) in detail would aid assumptions about both current and future policy options.

The research program will study the transfer of technology innovation to other countries. This research will help predict the flow of technology and the effectiveness of various instruments to facilitate the transfer. The research will help decision makers understand how to hoth encourage the transfer so that global climate goals can be met by developing countries and maintain intellectual property rights.

Greenhouse-Gas Indices. Policy instruments to decrease greenhouse-gas emissions that apply to more than one gas need a formula for trading off among the gases. This research is concemed with many scientific and economic issues, including the expected lifetime of the different gases, their relative absorbing capacities, the accounting of 
sources and sinks, the resulting atmospheric concentrations over time, estimates of damage that vary over time, and credits or debits for other economic effects (for instance, CFCs as contributors to ozone depletion and $\mathrm{CO}_{2}$ increases that can enhance some crops).

\section{Program Manager}

John C. Houghton

Environmental Sciences Division

Department of Energy, ER-74

Washington, DC 20585

(301) 903-8288

Internet:

john.houghton@mailgw.er.doe.gov 


\section{Research Summaries}

\section{TRADEOFFS AMONG GREENHOUSE-GAS EMISSIONS: AN EQUIVALENT-DAMAGE APPROACH}

\author{
ADAMS, JOHN L. \\ RAND

$\begin{array}{lr}\text { FY } 1993 & 145 \\ \text { FY } 1992 & 0 \\ \text { FY } 1991 & 0\end{array}$

09/30/93-09/29/94

Objective: To investigate the development of greenhouse-gas indices (GGI) that vary with time and provide an economically efficient solution for a variety of emission scenarios and damage functions.

Product: A GGI system that will provide a basis for assessing the importance of various inputs.

Approach: An equivalent-damage index (EDI) will be developed for evaluating the effects of changes in the mixture and timing of anthropogenic greenhouse-gas emissions. It will be based on the economic damages associated with emissions of a particular greenhouse gas (e.g., $\mathrm{CO}_{2}, \mathrm{CH}_{4}, \mathrm{~N}_{2} \mathrm{O}$, or CFC-12) at a specified date. The dependence of EDI values on the qualitative relationship between climate change and economic damages and on the time paths of anthropogenic greenhouse-gas emissions will be evaluated. In addition, the sensitivity of EDI values to current uncertainties in the climatechange process (e.g., climate sensitivity and atmospheric residence tumes) will be assessed.

The EDI will provide a basis for decentralized regulation of greenhouse-gas emissions, including international agreements and national regulatory programs. With the index, nations, firms, and other agents can determine least-cost measures for limiting their own contributions to climate change, and emission-permit systems (within or between nations) can be structured to allow trading across greenhouse gases and across time. The sensitivity of index values to damages, future emissions, and other factors will determine the extent to which such decentralized regulatory approaches can reduce the costs of abating climate change.
An improved emission/atmospheric-concentration submodel, based on research and input from Lawrence Livermore National Laboratory, will be constructed and integrated. This submodel will calculate changes in atmospheric greenhouse gas concentrations, including representation of carbon-cycle and atmospheric-feedback effects, chemical interactions with $\mathrm{O}_{3}$, and the impact of these effects on radiative forcing, surface temperature, and sea level. The changes in global mean surface temperature will be simulated with an upwellingdiffusion ocean model. Sea-level rise will be calculated by including thermal expansion and melting of glaciers and ice sheets.

Results to Date: New project.

\section{INVESTMENT IN NEW TECHNOLOGIES: AN EXPERIMENTAL APPROACH}

BJORNSTAD, DAVID J.

OAK RIDGE NATIONAL LABORATORY

$\begin{array}{lr}\text { FY } 1993 & 146 \\ \text { FY 1992 } & 0 \\ \text { FY } 1991 & 0\end{array}$

07/01/93-09/30/92

Objective: To explain the discrepancy between top/down and bottom/up modeling and to conduct controlled laboratory experiments to test the implications of new literature, such as that including "irreversibility."

Product: An understanding of what drives investments in technologies that are important to global, warming.

Approach: When will firms invest in technologies that reduce CFCs or other greenhouse-gas emissions? Traditional economic theory suggests that investment will occur when the expected return on the investment is at least equal to the cost of capital (the interest rate). However, a new literature has developed to explain why investment seems to require a rate of return greater, then the traditional cost-of-capital level.

Results to Date: New project. 


\section{BARRIERS TO ENERGY-TECHNOLOGY ADOPTION: AN ANALYSIS OF EVIDENCE FROM SELECTED DSM PROGRAMS IN THE U.S.}

DOWLATABADI, H.; FISCHHOFF, B.; and LAVE, L. B.

\section{CARNEGIE-MELLON UNIVERSITY}

\author{
FY 1993 \\ FY 1992 \\ FY 1991
}

133

0

0

\section{INVERSE METHODS FOR \\ PARAMETERIZATION OF ENERGY-USE MODELS: AN APPLICATION OF BAYESIAN UPDATING TO THE EDMONDS-REILLY MODEL}

DOWLATABADI, H., and SMALL, $H$.

CARNEGIE-MELLON UNIVERSITY

$\begin{array}{lr}\text { FY } 1993 & 131 \\ \text { FY 1992 } & 0 \\ \text { FY 1991 } & 0\end{array}$

$09 / 15 / 93-09 / 14 / 94$

Objective: To estimate the value of the autonomous energy-efficiency index (AEEI), which represents how much less energy is used per unit of GDP and which measures the degree to which energy will be used more efficiently as technical advances occur.

Product: A method for estimating the AEEI that combines Monte Carlo methods with Bayesian updating techniques.

Approach: Several models and data for the past several decades will be used to backcast a value of the AEEI with Bayesian statistics. For these models, the inputs are less well known than the outputs. A prior distribution will be assumed for each input, Monte Carlo simulation will be used to generate input values, the model will be run to produce outputs, the outputs will be screened against actual values, and a posterior distribution will be inferred on the input variables. This prodedure will develop both new estimates of the input variables (such as the AEEI) and estimates of the correlation of AEEI with other related input variables (such as labor productivity and GDP growth).

Two alternative model structures will be examined: one with uncorrelated input parameters and the other with correlated input parameters. The Bayesian updating techniques will use likelihood-based windows constructed from actual observations of the output variables to filter out the model simulations that do not conform with the observed values. Two types of windows will be used: binary windows and probabilistic windows. Output distribution parameters will be calculated to measure the effects of windowing on the output distributions (in the binary case). The partial rank correlation structure of the inputs and outputs will also 
be determined. The prior distributions and correlation structure of the inputs will be revised through the updating process. An application of the windowing process will illustrate the impacts of a restrictive policy on $\mathrm{CO}_{2}$ emissions on the input structure.

Results to Date: New project.

\section{ECONOMICS AND INTEGRATED ASSESSMENT MODELING}

EDMONDS, JAMES A.

\section{PACIFIC NOR'THWEST LABORATORY}

$\begin{array}{lr}\text { FY 1993, } & 175 \\ \text { FY 1992 } & 0 \\ \text { FY 1991 } & 0\end{array}$

07/01/93-09/30/93

Objective: To support the Office of Energy Research in a broad spectrum of areas related to energy and greenhouse-gas emissions.

Product: Improved quality of the Economic Research Program, a better understanding of autonomous energyefficiency indices (in particular, their relationship to integrated-assessment models), and U.S. support of the IPCC assessment report.

Approach: Services will be provided on an as-needed basis. PNL and the Project Monitor will jointly determine the specific tasks to be accomplished, along with specific approaches to those tasks. Those services will include:

- Support of authors for the Working Group III of the Intergovernmental Panel on Climate Change (IPCC)

- Conducting a workshop on future research priorities

- Conducting a workshop to present the latest advances in industrial organization to serve as a conceptual foundation for integrated-assessment modeling

- Investigating the nature of autonomous energyefficiency indices for innovation as it applies to global climate change
- Providing support to the Assessment Working Group of the Global Climate Change Program, Committee on Earth and Environmental Sciences (CEES)

Results to Date: New project.

\section{ENERGY AND FUTURE CARBON DIOXIDE EMISSIONS}

EDMONDS, JAMES A.

\section{PACIFIC NORTHWEST LABORATORY}

$\begin{array}{lr}\text { FY } 1993 & 50 \\ \text { FY } 1992 & 0 \\ \text { FY } 1991 & 0\end{array}$

07/01/93-09/30/93

Objective: To improve DOE's Economics of Global Change Research Program.

Product: An improvement in the quality of the Economics of Global Change Research Program.

Approach: Services will be provided to the Office of Energy Research on an as-needed basis. These services will include, for example, providing advice to and assisting the Research Program on Economics of Global Change by conducting briefings on state-of-the-art topics in integrated global-change assessment, participating in the proposal evaluation process, chairing panel review sessions, and participating in various working groups of the CEES and Working Group 3 (WG3) of the Intergovernmental Panel on Climate Change.

Results to Date: New project.

GLOBAL CLIMATE CHANGE AND THE

SOCIAL SCIENCES: AN INTERNATIONAL ASSESSMENT OF TECHNOLOGY

EDMONDS, JAMES A.

PACIFIC NORTHWEST LABORATORY

$\begin{array}{lr}\text { FY } 1993 & 75 \\ \text { FY } 1992 & 0 \\ \text { FY } 1991 & 0\end{array}$

07/01/93-09/30/93 
Objective: To assess the human processes associated with technology and to increase our understanding of global climate change and the responses and policy issues that are related to global climate change.

Product: A stand-alone assessment of the state of knowledge about the introduction, penetration, and removal of technologies related to global climate change.

Approach: A collaborative approach will be used to develop a credible and truly international assessment of the development, adoption, and replacement of technological tools and processes. A principal author will be recruited together with several lead authors and/or contributing authors. PNL will manage the overall group processes. At least one workshop will be held for all members of the group to plan, resolve issues, and provide mutual feedback. The principal author, in collaboration with PNL, will be responsible for producing a unified draft assessment. A completed draft will be ready for review on or before September 30, 1994.

Results to Date: New project.

\section{APPLYING EVOLUTIONARY ALGORITHMS TO REPRESENT LONG-RUN TECHNOLOGY ADOPTION IN GLOBAL-CHANGE MODELS}

LEIBY, PAUL $N$.

OAK RIDGE NATIONAL LABORATORY
$\begin{array}{cr}\text { FY } 1993 & 168 \\ \text { FY } 1992 & 0 \\ \text { FY } 1991 & 0\end{array}$

07/01/93-09/30/92

Objective: To address the limitations of traditional energy-economic models in evaluating long-term change and technology choice.

Product: A modeling framework (and a demonstration of its application) that could serve as the basis for extensions to existing integrated global change modeling systems.

Approach: Ideas from several disciplines will be combined: computable market equilibrium modeling in energy-economics, individual-based ecological modeling in biology, and genetic algorithms in artificial intelligence. The research will apply evolutionary modeling methods to the problem of characterizing long-run technological change and diffusion in energy/economic systems. The result will extend existing integrated global change modeling systems by breaking away from neoclassical economics paradigms.

Results to Date: New project.

\section{INTERNATIONAL WORKSHOP ON INTEGRATIVE ASSESSMENTS OF MITIGATION OF, IMPACTS OF, AND ADAPTATION TO CLIMATE CHANGE}

NAKICENOVIC, NEBOJSA

\section{INTERNATIONAL INSTITUTE FOR APPLIED SYSTEMS ANALYSIS}

$\begin{array}{lr}\text { FY } 1993 & 20 \\ \text { FY } 1992 & 0 \\ \text { FY } 1991 & 0\end{array}$

09/30/93-09/29/94

Objective: To bring together integrated-assessment modelers from around the world to discuss such modeling with an emphasis on non-US-based models.

Product: A fuller understanding of the economic effects of climate change with special emphasis on adaptation strategies and comparisons of mitigation, adaptation, and impacts assessments.

Approach: Each of the four half-day sessions will be initiated with keynote presentations from the small panel of prominent discussants who will then lead the general discussions. The opening session will have two keynote speakers, addressing policy issues and research questions. Sessions will be held on methodological issues, integrative assessment, mitigation, impacts, and adaptation. The presented papers and key discussion points will be published in a timely manner.

Results to Date: New project. 


\section{ECONOMICS OF GLOBAL CHANGE RESEARCH PROGRAM}

\author{
PRINN, RONALD G., and JACOBY, HENRY \\ MASSACHUSETTS INSTTTUTE OF \\ TECHNOLOGY
}

$\begin{array}{lr}\text { FY } 1993 & 116 \\ \text { FY 1992 } & 0 \\ \text { FY } 1991 & 0\end{array}$

09/15/93-09/14/94

Objective: To develop a system of greenhouse-gas indices to guide policy-makers in the search for efficient solutions to emission containment. The indices will promote the trading off among the different greenhouse gases, allowing the market to generate a least-cost solution. Developing indices is a complex issue that combines the natural sciences, economies, and other social sciences.

Product: A comprehensive approach to the development of greenhouse-gas indices.

Approach: The researchers will identify the salient criteria for the theoretical success of such an index, including the chemical and climate feedbacks, the latitudinal distribution of emissions, variable emission scenarios, and a sophisticated damage function. Greenhouse-gas indices will require the integration of a large number of natural science and economic factors, and the best way to develop such an index will be through the use of an integrated-assessment model. Nonparametric damage functions will be used in the first year. In the second year, the restrictions necessary for the nonparametric damage functions and disaggregate emissions forecasts by latitude, if necessary, will be relaxed.

Results to Date: New project.

\section{UNCERTAINTY IN AN INTEGRATED MODEL OF GREENHOUSE WARMING: THE DIFFERENTIAL VALUE OF DIFFERENT INFORMATION UNDER ALTERNATIVE VALUATION METRICS}

SOUTH, D.

ARGONNE NATIONAL LABORATORY
$\begin{array}{cc} & \\ \text { FY } 1993 & 69 \\ \text { FY } 1992 & 0 \\ \text { FY } 1991 & 0\end{array}$

07/01/93-09/30/93

Objective: To investigate the value of reducing uncertainty and the value of waiting or not waiting before abating the threat of global climate change.

Product: The integration of an updated version of the Nordhaus-Yohe probabilistic model for global carbon emissions with updated damage schedules through a modified, stochastically framed, Nordhaus emissions-toimpacts model.

Approach: The various sources of uncertainty in integrated models will be ranked according to their importance in producing overall variance in the estimated "best" abatement strategy (reflected by the efficient shadow price of carbon). The robustness of results produced from more disaggregated but less flexible models will then be assessed before the values of reducing uncertainty at each source, assessed in terms of at least two social valuation metrics, are explored. Estimates of the socially weighted, "interesting" scenarios upon which best to conduct future, moredetailed analysis will be suggested. The research will improve Nordhaus-Yohe integrated assessment models by updating the marginal damage schedule and adding stochastic correlations between changes in atmospheric concentrations and temperature.

Results to Date: New project. 


\section{TECHNOLOGY TRANSFER AND DEVELOPMENT FOR REDUCING GLOBAL GREENHOUSE-GAS EMISSIONS: INCENTIVES, INSTITUTIONAL PROBLEMS, AND SOLUTIONS}

TOMAN, MICHAEL A.

RESOURCES FOR THE FUTURE

$\begin{array}{lr}\text { FY } 1993 & 100 \\ \text { FY 1992 } & 0 \\ \text { FY } 1991 & 0\end{array}$

$09 / 15 / 93-09 / 14 / 94$

Objective: To investigate the process of innovatin $;$ and particularly transferring technology and why it happens and to hetter explain the strategic trade-related implications (e.g., intellectual property rights) of innovation expenditures.

Product: A fuller understanding of the theoretical, empirical, and institutional/policy questions related to the development and international transfer of technologies that can reduce the growth rate of greenhouse-gas emissions.

Approach: Data will be collected, and conceptual models will be developed to test and investigate the new theories in international trade that have appeared in the literature during the past two decades. These models will then be applied to global-climate-change concerns to unite some of the theoretical advances with the practical issues of including them in energy models.

Three primary forces that shape the transfer of technology will be investigated: incentives for adoption, transfer of proprietary rights, and the creation of new technologies. The incentives for adoption are internal to the receiving country. Subsidized energy costs and lack of environmental regulation are distortions that serve to discourage adoption. Other issues that will be studied include the fit of the technology to the receiving country, capital markets, and various governmental policies. The fundamental organizational influences (in markets, legal systems, and policies) on technology transfer and the resulting implications for incentives to innovate will be examined.

Results to Date: New project.

\section{TECHNICAL INNOVATION AND DIFFUSION IN' LARGE-SCALE ENERGY MODELS}

WEYANT, J. P., and HUNTINGTON, H. G.

\section{STANFORD UNIVERSITY}

$\begin{array}{lr}\text { FY } 1993 & 99 \\ \text { FY } 1992 & 0 \\ \text { FY } 1991 & 0\end{array}$

$09 / 15 / 93-09 / 14 / 94$

Objective: To recommend changes in integratedassessment models of global climate change, to evaluate how existing top-down models and bottom-up analyses represent new technical innovation and diffusion, and to augment and to build upon recent studies of global climate change and energy conservation.

Product: A merger between advances in investment and diffusion analysis appearing in the economics literature on the one hand with the modeling and analysis of energy technologies and energy market adjustments on the other.

Approach: Because the autonomous energy-efficiency index (AEED) is one of the most sensitive parameters of integrated-assessment models and the parameter that modelers know the least about, that index and its relationship to the top-down and bottom-up paradigms of technology innovation and diffusion will be investigated. Various conceptual approaches to analyzing investment and diffusion will be reviewed, including some of the more recent work on irreversibility and illiquidity of investments. A taxonomy will be developed to differentiate energy investments from other investments to determine whether specific economics research results generalize to the energy area. A stylized model will then be built to assess how existing topdown and bottom-up models represent technology and innovation. The feasibility of including the results from the previous tasks in existing energy models will be evaluated, with the stylized model simulating the technology-innovation and -diffusion aspects of the large-scale model.

Results to Date: New project. 


\section{COUNTRY STUDIES}

WILLIAMS, EDWARD R.

\section{OFFICE OF POLICY, PLANNING, AND PROGRAM EVALUATION}

$\begin{array}{lr}\text { FY } 1993 & 984 \\ \text { FY } 1992 & 0 \\ \text { FY } 1991 & 0\end{array}$

$$
04 / 01 / 93-03 / 31 / 94
$$

Objective: To enhance the capabilities of countries and/or regions to inventory their net emissions of greenhouse gases, to assess their vulnerabilities to climate change, and to evaluate the options available to them to mitigate and adapt to climate change. To support countries' efforts to develop and implement national policies and measures. To develop data and information that can be used at the national, regional, and global levels to assess current and future trends in net anthropogenic emissions of greenhouse gases and to further national and international discussions of climatechange issues.

Product: Country studies are expected to improve the recipient's capacity to carry out similar analyses and develop products, usually in the form of a well-documented report that would be distributed to the international community (e.g., the IPCC). The studies should help countries develop a basis for formulating and updating national programs containing measures to mitigate climate change and measures to facilitate adequate adaptation to climate change. The formulation and updating of these programs is required by the Framework Convention on Climate Change.

Approach: This initiative will be directed by a committee made up of representatives of various U.S. Government agencies, including DOE. The day-to-day operations will be managed by a country-studies management team, composed of U.S. Government personnel. The team will convene project teams to assist in the development and review of proposals and to support the studies.

Results to Date: New project.

\section{REDUCED-FORM ATMOSPHERIC MODELS TO SUPPORT HAMMITT'S INTEGRATED- ASSESSMENT MODELING}

WUEBBLES, D. J.

\section{LAWRENCE LIVERMORE NATIONAL LABORATORY}

$\begin{array}{lr}\text { FY } 1993 & 50 \\ \text { FY } 1992 & 0 \\ \text { FY } 1991 & 0\end{array}$

07/01/93-09/30/93

Objective: To improve the treatment of science in the integrated-assessment model, building upon the economics modeling that is being done at Rand and allowing emissions to be translated into effects on climate.

Product: Reduced-form atmospheric models that include the carbon-cycle- and atmospheric-feedback effects, chemical interactions, radiative forcing, and surface-temperature and sea-level change for inclusion in the Rand integrated-assessment modeling.

Approach: The science modules will be designed to allow calculation of the greenhouse-gas-concentration changes based on input emissions (including representation of carbon-cycle and atmospheric-feedback effects), expected chemical interactions of ozone and other constituents, the resulting effects on radiative forcing, and the resulting effects on surface-temperature and sea-level changes.

Results to Date: New project.

ENDOGENOUS TECHNOLOGICAL PROGRESS IN A LONG-RUN MODEL OF THE ECONOMY AND CLIMA'TE CHANGE

YANG, ZILI

\section{OAK RIDGE NATIONAL LABORATORY}

$\begin{array}{lr}\text { FY } 1993 & 156 \\ \text { FY } 1992 & 0 \\ \text { FY } 1991 & 0\end{array}$

07/01/93-09/30/92 
Objective: To characterize the linkages between technolugical progress and the economy, to simulate technology transfer in a two-region framework, and to investigate the relationship between human capital and technological advances.

Product: An extended version of Nordhaus's integratedassessment model, DICE, that includes explicit, endogenous components to disaggregate the impacts of "autonomous" improvements, mitigation, adaptation, and economic productivity on changes in technology.

Approach: Technological change will be explicitely incorporated into a model of the economy and climate change. Nordhaus's Dynamic Integrated ClimateEconomy (DICE) model will be enriched by addressing different ways in which endogenous technological change can be embodied within the model. The study will focus on the role of technological advances that would impact the level of uncontrolled greenhouse-gas emissions, the ability to mitigate emissions, the development of nonfossil-fuel technologies, the ability to adapt to potential changes in climate, and economic productivity. Each of these five concerns will be an explicit, endogenous component of the enhanced model. In addition, the modeling effort will address interregional relationships, free-rider phenomena, and the conditions under which it may be strategically advantageous for some regions to engage in unilateral technology transfer to other, less-developed regions. The role of human capital and its effects on increasing shortterm production will also be explored.

Results to Date: New project. 


\section{GLOBAL CHANGE EDUCATION}

Numerous studies have identified the need for well-trained scientists and engineers to advance progress in science, technology, and global competitiveness. During FY 1991, the Department of Energy (DOE) initiated a Global Change Education Program (GCEP) to strengthen the human resource base in science and technology and to provide members of the Federal Coordinating Council on Science, Engineering, and Technology's Committee on Earth and Environmental Sciences (CEES) with highly trained and educated individuals for advancing the cross-disciplinary science of global change. The major emphasis of this education program has been to award competitive postdoctoral and graduate-level fellowships for training the next generation of scientists with the interdisciplinary skills necessary for conducting globalchange research.

Since the program began, DOE has awarded 38 two-year postdoctoral fellowships and 64 four-year graduate-level fellowships. Currently, 26 postdoctoral fellows are conducting research at $21 \mathrm{DOE}$, university, and other CEES-agency laboratories, which allows them to gain access to outstanding professional staffs and state-of-the-art scientific equipment. Currently, the DOE GCEP is also supporting the tuition, fees, and stipend to train and educate 60 graduate students at 31 universities with the scientific skills necessary for conducting global-change research. The DOE GCEP requires these graduate fellows to spend at least 6 weeks at CEES-agency laboratories to acquaint themselves with ongoing multidisciplinary research programs in global change.

The DOE GCEP has been developed and implemented in concert with the USGCRP and therefore addresses the objectives, activity streams, and science elements of the Global Change Research Plan. Both postdoctoral and graduate-level fellows are encouraged to interact with the scientific staff of the various CEES-agency laboratories. About one-thind of the DOE postdoctoral fellows are conducting research at NASA, NOAA, and USDA laboratories, and the remaining two-thirds are at university and DOE laboratories. Of the $45 \mathrm{DOE}$ graduate fellows who have completed their sixweek research experience at CEES-agency laboratories, more than half were at NASA, NCAR, NOAA, USGS, USDA, and other agency laboratories. The DOE CGEP emphasizes a wide suite of sciences, laboratories, and stakeholders because the global change problem, like many challenges in industry, is exacerbated by the need for crossdisciplinary training.

Broad-based education and training strengthens the interdisciplinary problem-solving and modeling skills that are necessary for addressing science, technology, and policy issues. During FY 1993, the DOE GCEP is sponsoring a workshop, New Research in the Science of Global Change: A Multidisciplinary View that will provide a forum for the DOE postdoctoral fellows to begin assessing their research results and addressing these issues. Also during this fiscal year, the first DOE graduate fellow to finish a doctoral dissertation has accepted a directorship position with The Nature Conservancy. As more of these graduate fellows receive their doctoral degrees and apply their cross-disciplinary and problem-solving skills within the science of global change, our nation's human-resource base in science and technology will be enhanced, and our policy decisions to ensure the future integrity of the Earth's environment will benefit. 


\section{Program Manager}

\section{Curtis R. Olsen}

Environmental Sciences Division

Department of Energy, ER-74

Washington, DC 20585

(301) 903-5329

Internet:

curtis.olsen@mailgw.er.doe.gov 


\section{Research Summaries}

\author{
GRADUATE FELLOWSHIPS FOR GLOBAL \\ CHANGE \\ WOHLPART, A.

\section{OAK RIDGE INSTITUTE FOR SCIENCE AND EDUCATION}

\begin{tabular}{lr} 
FY 1993 & 1650 \\
FY 1992 & 0 \\
FY 1991 & 0 \\
& \\
\multicolumn{1}{|c}{$10 / 01 / 92-09 / 30 / 93$}
\end{tabular}

Objective: To help ensure an adequate supply of professionals with advanced degrees to implement global change research identified by the Committee on Earth and Environmental Sciences (CEES).

Product: Professionals with advanced degrees who are educated and trained in the seven interdisciplinary science areas of global change to serve as the teachers and researchers of the future.

Approach: U.S. citizens who hold at least a baccalaureate degree in a discipline pertinent to global change will submit their applications to the program in response to a nationwide advertisement. Universities will also submit their applications for designatedparticipating status. A panel of representatives from the academic community and DOE will evaluate the student and university applicants, and recommend to Oak Ridge Institute for Science and Education, for transmission to and approval by DOE, those students and universities that meet the criteria of the program.

Fellows will matriculate at DOE designated-participating universities and participate in a required collaborative research experience (RCRE) at either a DOE or other government agency research facility involved in globalchange research. The RCRE will provide the opportunity for the fellows to experience first-hand the working environment at a multidisciplinary research facility, to interact with the scientific staff of the facility, and to access state-of-the-art equipment and facilities. The fellows' participation in the RCRE will help to strengthen the interaction between the research facilities and the academic community.
Results to Date: Since its inception in FY 1991, the program has appointed 65 fellows, 61 of whotn were supported during FY 1993. Also, 64 universities have been awarded designated-participating status.

\section{POSTDOCTORAL RESEARCH PROGRAM FOR GLOBAL CHANGE}

WOHLPART, A.

\section{OAK RIDGE INSTITUTE FOR SCIENCE AND EDUCATION}

$\begin{array}{ll}\text { FY } 1993 & 1150 \\ \text { FY } 1992 & 2310 \\ \text { FY } 1991 & 1904\end{array}$

$10 / 01 / 92-09 / 30 / 93$

Objective: To increase the involvement of scientists and engineers in areas supportive of the U.S. Global Change Research Program.

Product: A pool of professionals with the experience necessary for future research and teaching.

Approach: Recent doctoral-degree recipients will conduct research in support of the USGCRP as it relates to the specific mission of DOE and the Subcommittee on Global Change Research of CEES. Fellows will spend up to two years at approved laboratories (DOE, university, or other), conducting research in technical areas related to the strategic priorities of the USGCRP. Fellows will gain access to a valuable combination of outstanding professional staff, scientific equipment and facilities, and ongoing global-change research. These fellowships will help promote the influx of new ideas and skills into the laboratories, complement and extend the traditional academic research and training programs, and enhance interactions between the academic community and federal researchers.

Results to Date: Following a variety of promotional activities for the FY-1993 cycle of program operation, 109 applications were received. These applicants requested appointments at 60 laboratories: 9 DOE, 26 university, 1 private, and 24 sponsored by other federal 
departments and agencies. They received their doctoral degrees from 61 U.S. and foreign universittes. Of the total applicant pool, 103 were reviewed by a panel of 11 distinguished scientists. The panel identified 22 finalists, and DOE selected 12 to recelve awards.

The 12 fellows, 2 of whom are female, recelved their doctoral degrees from 11 universities in a variety of disciplines: astronomy, blology, chemistry, ecology, geology, meteorology, microbiology, physics, and plant physiology. They will serve their appointments at four DOE and six university laboratories as well as one NASA and one NOAA laboratory. Their research will focus on the following global change-related toples: effects of elevated $\mathrm{CO}_{2}$; the variance and heat flux of cyclones; soll carbon inventories; biological invasions; population density; atmospheric nitric oxide; solar variability; cloud properties; ice in high clouds; carbon assimilation; and methyl bromide cycling.

In addition to the 12 new awardees, the 12 fellows appointed during FY 1992 are continuing their research at live DOE and four universily laboratories as well as one NASA, one USDA, and one NOAA laboratory. Five have renewed their appointments; seven will be offered renewals during the first quarter of FY 1994. Of the 14 fellows appointed in FY 1991, 5 have terminated; 2 more will terminate In FY 1993; and the remaining 7 will terminate during the first quarter of FY 1994. The deadline for the next cycle will be February 15, 1994.

The program sponsored a workshop, entitled New Research in the Science of Global Change: A Multidlsciplinary View, in September 1993 in Oakland, Calif. The workshop was designed to provide fellows with an opportunity to share creative ideas, make valuable contacts, and learn about the multidisciplinary aspects of global-change research. The fellows presented their research, participated in a field trip to Lawrence Livermore National Laboratory, and heard keynote speakers. The fellows' mentors, the coordinators of host laboratories, and representatives of federal departunents and agencies participating in the USGCRP were invited to altend. 


\section{INFORMATION AND INTEGRATION}

Knowledge is based on analysis and evaluation of information. Knowledge is of little use if the persons and organizations needing it are not aware of it, cannot obtain it, or cannot be sure of the quality of the information on which it is based. The Olobal Change Research Program explores scientific issues that are comprehensive and complex. The need for effective communication among scientists, policymakers, educators, students, corporate officials, and the interested public is vital.

Objectives. The information and integration component of the Olobal Change Research Pro. gram serves as a scientific interface through which technical information can be obtained, evaluated, quality-assured, and distributed; the exchange of data can be promoted and facilitated; and high. quality analyses of complex data can be performed to synthesize information used in evaluating environmental issues. Specific objectives of the information and integration component include promotion of networking among members of the global-change community, preparation of technical and informational reports, and sponsorship of scientific conferences. The Olobal Change Research Program provides access to current global-change information and data through the Carbon Dioxide Information Analysis Center (CDIAC), which produces quality-assured and fully-documented numeric data, distributes technical publications, and produces newsletters and research summaries. CDIAC also responds to inquiries from an audience representing broad interests for information on a wide variety of global-change topies.

\section{Program Manager}

Thomas J. Gross

Environmental Sciences Division

Department of Energy, ER-74

Washington, DC 20585

(301) 903-3316

Internet:

thomas.gross@mailgw.er.doe.gov 


\section{Research Summaries}

\section{CARBON DIOXIDE INFORMATION ANALYSIS CENTER}

CUSHMAN, ROBERT

OAK RIDGE NATIONAL LABORATORY

$\begin{array}{ll}\text { FY } 1993 & 2100 \\ \text { FY 1992 } & 2050 \\ \text { FY 1991 } & 1907\end{array}$

$10 / 01 / 91-09 / 30 / 92$

Objectlve: To acquire or compile, quality-assure, document, archlve, and distribute data and other information concerning $\mathrm{CO}_{2}$, other greenhouse gases, and climate change in support of DOE's Olobal Change Research Program (GCRP).

Product: A fully integrated information analysis center needed to support OCRP's research program and to provide data and other information conceming $\mathrm{CO}_{2}$, other greenhouse gases, and climate change to the public and to the research, policy-making, education, and corporate communities.

Approach: The Carbon Dioxide Information Analysis Center (CDIAC) will identify users' needs by working closely with the research community, the Global Change Research Program, policymakers, and others by attending workshops, reviewing the literature, and maintaining personal contacts. Data, models, and other products and services will be evaluated as to usefulness and acquired if available or compiled at CDIAC if not in a usable form.

These products will undergo extensive quality assurance and complete documentation in full coordination with the original supplier of the information. They will then be made available to requesters. In addition to data packages, CDIAC will also produce and distribute newsletters, research summaries, and glossaries. CDIAC will work with other data centers and individual researchers to promote the compilation and exchange of data. CDIAC's activities and approaches will evolve with changing research needs and technology, and future emphasis will be placed on exploring new methods of communication, new media for archiving and distribution, and new data products.

CDIAC's research activities will reflect any new programmatic directions of GCRP. CDIAC's activities will include information support for the evaluation of complex environmental issues associated with elevated levels of atmospheric trace gases.

Results to Date: CDIAC has provided technical support for the Global Change Research Program; the general research, policy-making, education, and corporate communities; and the public by serving as GCRP's information-gathering and distribution center. Activities included obtaining and evaluating data and technical literature; producing numeric data packages (NDPs) and computer model packages (CMPs); and distributing $\mathrm{CO}_{2}$-related reports. During FY 1993, seven new or updated NDPs were produced (topics included oceanographic $\mathrm{CO}_{2}$ and $\mathrm{N}_{2} \mathrm{O}$ measurements, climate data from the former USSR, $\mathrm{CO}_{2}$ emission estimates from fossil-fuel burning, atmospheric methane and isotopic methane measurements, historic land-use and carbon estimates for South and Southeast Asia, and data for assessing the vulnerability of the U.S. Gulf Coast to sea-level rise). Eight issues in the DOE Research Summary series were produced, and the newsletter CDIAC Communications was published. CDIAC issued a two-disk CD-ROM, CDIAC's Numeric Data Package Collection. CDIAC stalf co-authored (with scientists at the Chinese Academy of Sciences, the State University of New York, and the National Climatic Data Center) the report Climate Data Bases of the People's Republic of China. In addition, CDIAC produced the Catalog of CDIAC Numeric Data Packages and Computer Model Packages.

In the first half of FY 1993, CDIAC responded to 514 requests from 46 countries for NDPs and CMPs. Since FY 1985, 8137 copies of data sets and models have been distributed. CDIAC filled 3620 requests from 1260 individuals in 58 countries in the first half of FY 1993. This brings CDIAC's total to 40,836 requests from users in 104 countries since FY 1985. 
PARTIAL SUPPORT FOR THE U.S. NATIONAL COMMITTEE FOR CODATA AND COMMITTEE FOR AN IGBP PILOT STUDY ON DATABASE INTERFACES

UHLIR, PAUL F.

\section{NATIONAL ACADEMY OF SCIENCES}

$\begin{array}{lr}\text { FY } 1993 & 25 \\ \text { FY } 1992 & 23 \\ \text { FY } 1991 & 0\end{array}$

$09 / 15 / 93-09 / 14 / 94$

Objectlve: To improve the quality and accessibility of data, to facilitate international cooperation, and to promote an increased awareness of the importance of these activities.

Product: Improved quality and accessibility of all types of quartitative data resulting from experimental measurements or observations in the physical, biological, geological, and astronomical sciences.

Approach: At least two meetings will be held to receive briefings and to consider requests for advice from sponsors and other government agencies, to provide advice on the U.S. position on data-related issues, to provide oversight for the IGBP Pilot Study on Database Interfaces, and to receive briefings and other information about access to data issues. The IGBP Pilot Study on Database Interfaces will publish its report in the fall of 1993. CODATA newsletters, bulletins, and other data-related information will be disseminated. 


\section{NATIONAL INSTITUTE FOR GLOBAL ENVIRONMENTAL CHANGE}

The House Appropriations Subcommittee on Energy and Water established and provided the resources for the National Institute for Global Environmental Change (NIGEC) on September 29, 1989. The purpose was to contribute to the knowledge base of global climate change, the reduction of key scientific uncertainties inherent in the projections of future climate states, and the perturbations attributed to man's activities. NIGEC's mission is to support the sponsoring agency, DOE, within the federal Global Change Research Program, coordinating its activities with those of ARM and other DOE efforts.

NIGEC has emphasized academic research programs dealing with the scientific aspects of global environmental change but has also seen some attention to research undergirding the development of policy strategies and options and to educational efforts. The policy-related work seeks the practical application of science in the pursuit of appropriate management of anticipated or possible climatic conditions of the 21 st century.

The mission of NIGEC in support of global-change research within DOE objectives has had some change as NIGEC and other research arms of that department have been coordinated. During the past year, NIGEC has been operating under the guidelines established by DOE and expressed in five priority questions, to wit:

1. What is the radiative forcing from greenhouse gases, aerosols, and clouds, and what are the feedbacks and processes that regulate the net radiative balance of the Earth's atmosphere? How can climate models be improved to provide greater spatial resolution and morerealistic representation of the processes that influence radiative balance of the atmosphere, particularly the effects of clouds, aerosols, and gaseous radiative effects?

2. How can human-induced climatic changes be unequivocally detected in the existing climate record?

3. What are the major processes controlling the increase in atmospheric $\mathrm{CO}_{2}$; in particular, what are the net carbon exchanges with the atmosphere for major terrestrial and marine ecosystems, and what factors regulate these exchanges? Also, what accounts for the recent rate of increase in atmospheric methane?

4. How do terrestrial organisms and ecosystems respond to elevated $\mathrm{CO}_{2}$ concentrations and to potential changes in climatic conditions (i.e., temperature and moisture)? What regulates these responses, and how can the responses and underlying controls be detected and quantified? How are the responses integrated across organizational levels to produce adjustments in terrestrial ecosystems to the changes?

5. What are the technological, economic, and social factors that drive the emission of radiatively active trace gases and aerosols, and what are the potential effects of global climate change on these factors? 
For FY 1993-1994, these questions still form an underlying guide, but changes in direction are to be anticipated and accommodated as the mission is altered.

Regional directors have been encouraged to develop coordinated research programs appropriate to their own regional resources and needs. This course of action is intended to take advantage of regional differences in climate and physical resources as well as of the availability of scientific research resources, including personnel and facilities, unique to the area. As a result, some regional programs are more specialized than others, and all are in a continuing state of program evolution, as indicated by the summaries of programs listed below and the individual project abstracts.

Great Plains Regional Center: Administers research projects on trace-gas dynamics in selected ecosystems; detection and attribution of the radiative effects of increases in greenhouse gases and aerosols, especially grassland and aquatic systems, during the past century; and physical, biological, and human consequences of climate change.

Midwestern Regional Center: Administers research projects on the identification of sources and sinks of trace gases and aerosols, especially those related to global warming and ozone depletion, effects of global climate change on terrestrial ecosystems, and technological and socioeconomic factors and effects on global climate change.

Northeast Regional Center: Administers research projects on the influence of gases and aerosols on the composition of the atmosphere, especially on forest ecosystems, and heat and moisture transport by dynamical processes in the context of the global climate and radiative atmosphere budget.
Southcentral Regional Center: Administers research projects on sources and sinks of atmospheric methane, climate-data analysis, and modeling of physical and dynamical processes of the climate system for prediction and detection of climate change.

Southeast Regional Center: Administers research projects on the structure and process rates of forest ecosystems, especially in southern pine and southern Appalachian hardwoods; atmospheric and marine modeling; sources and sinks of greenhouse gases and aerosols; effects of technological, economic, and social factors; and the documentation of the historic climate record.

Western Regional Center: Administers research projects on factors that influence the atmospheric concentrations of greenhouse gases, with an emphasis on geochemical studies, terrestrial hydrological factors that influence global climate change, and chemistry modeling or observational approaches of the dynamics of the atmosphereocean climate system.

In June 1993, NIGEC cosponsored a conference in Gainesville, Florida, on UV-B radiation and its effects on people and the biosphere with the University of Florida.

\section{Program Manager}

Jerry W. Elwood

Environmental Sciences Division

Department of Energy, ER-74

Washington, DC 20585

(301) 903-4583

Internet:

jerry.elwood@ıailgw.er.doe.gov 


\section{Research Summaries}

\section{Great Plains Regional Center}

\begin{abstract}
EFFECTS OF ALTERED SOIL MOISTURE AND TEMPERATURE ON SOIL COMMUNITIES, PRIMARY PRODUCERS, AND ECOLOGICAL PROCESSES IN GRASSLAND ECOSYSTEMS
\end{abstract}

\author{
BLAIR, JOHN M., and \\ TODD, TIMOTHY C.
}

\section{KANSAS STATE UNIVERSITY}

Changes in soil moisture and temperature resulting from predicted climate changes will impact primary producers, soil communities, and ecological processes in grassland ecosystems. Just how prairie grasses, soil organisms, and key soil processes respond to different soil moisture and temperature regimes will be determined, and the potential consequences of these responses for ecosystem function in light of the predictions of global climate change models will be identified. Naturally occurring gradients of soil moisture and/or temperature will be used at two very different scales as experimental treatments to address the controls that moisture and temperature impose on ecological processes in grasslands. At a regional scale, reciprocal transplants of large, intact soil cores and associated plants between sites with different climatic conditions will address the effects of altered soil moisture and temperature on selected response variables. At a local scale, comparisons of tallgrass prairie sites with different soil-moisture availabilities and temperatures will be made. Variables in both experiments include net primary production, plant biomass carbon and $\mathrm{N}$, litter decomposition rates, soil invertebrate numbers and composition, soil microbial biomass and activity, and soil carbon and nitrogen pools.

\section{SPACE-TIME LOCAL HYDROLOGY INFLUENCED BY CHANGING CLIMATOLOGY: DISAGGREGATION, PREDICTION, AND COMPARISON}

B ,ARDI, ISTVAN; MATYASOVSZKY, ISTVAN;
and DUCKSTEIN, LUCIEN

UNIVERSITY OF NEBRASKA-LINCOLN and UNIVERSITY OF ARIZONA
A method for estimating local/regional hydrological quantities reflecting climate change will be developed. The geographical areas to be investigated cover central Arizona, the Upper Rio Grande, the lower Platte River Basin in Nebraska, the Ruhr River Basin in Germany, and the Lower Danube Basin in Hungary. Preliminary results indicate a strong relationship between dailycirculation-pattem types and daily-summer-precipitation amounts in eastern Nebraska, where summer storms are typically convective, as they are in Arizona.

The occurrence, persistence, and transition probabilities of daily atmospheric circulation patterns will be analyzed, and then the probability distribution function of local hydrological variables will be conditioned on these patterns. This approach is an extension of the usual analysis of regional hydrological impacts of climate change, where the quite-uncertain hydrological outputs of GCMs are disaggregated to estimate regional hydrological events.

The more accurate and verifiable global time series of GCM-produced circulation patterns will be examined to predict a basin-scale space-time hydrological series.

The research will consist of three phases. In the first, a disaggregation and classification of circulation patterns will be sought; each type of pattern will then be related within a conditional framework to local space-time precipitation, runoff, temperature, evapotranspiration, and wind. Combination of these variables, especially temperature, evapotranspiration, and wind, will be used to define regional drought indices.

In the second, available GCM outputs will be used to define scenarios of possible daily time series of circulation patterns. The linkages established in the first phase will be used to predict space-time daily precipitation, temperature, wind, and regional droughts. Finally, the historical series of regional hydrologic events will be compared with the various predictions.

In the third, local hydrological effects of global climate change will be compared and evaluated for the five regions considered. 


\section{ASSESSMENT OF CLIMATE CHANGE ON A MIXED AGRICULTURAL AND FOREST LANDSCAPE ON THE NORTH AMERICAN GREAT PLAINS}

\author{
BRANDLE, JAMES R., and \\ EASTERLING, WILLIAM E.
}

\section{UNIVERSITY OF NEBRASKA-LINCOLN}

A method for estimating the consequences of possible future climate change on mixed agricultural and forest landscapes on the North American Great Plains will be developed. A field effort will quantify the microclimate impacts of linear forested systems (i.e., shelterbelts and riparian corridors) of significant ecological and economic value in this region under conditions of climate change. A mouling effort will examine the roles of these linear forested systems in the Great Plains in mitigating the effects of climatic change. Basic relationships between shelter and wind flow in controlled, replicated systems will be tested to expand these relationships to better describe and to predict the roles trees play in the Great Plains environments. Questions related to scaling-up information on biological systems for use in regional-level climat-change assessments will be addressed.

\section{OBSERVATIONAL AND NUMERICAL STUDY FOR INTERANNUAL AND INTERDECADAL VARIABILITIES OF THE ATMOSPHERIC CIRCULATION}

\author{
CHEN, TSING-CHANG, and \\ TRIBBIA, JOSEPH J.
}

\section{IOWA STATE UNIVERSITY and NATIONAL CENTER FOR ATMOSPHERIC RESEARCH}

The interannual and interdecadal variability of the climate system will be examined with Version 1 of the NCAR CCM, which has been modified to run on computers at Iowa State University. Effects of the North Pacific sea-surface temperature (SST) variation on the interannual variability of the winter (DecemberFebruary) atmospheric circulation in the North PacificNorth America region will be examined. The effect of the Pacific SST variation on the interannual variability of the summer (June-August) atmospheric circulation will also be examined. The SST of the Comprehensive Ocean-Atmosphere Data Set (COADS) will be used in numerical simulations.

\section{REGIONAL PROJECTIONS OF CARBON DYNAMICS WITH GLOBAL CHANGE IN THE CENTRAL UNITED STATES}

\author{
ELLIOTT, EDWARD T., and \\ COLE, C. VERNON

\section{COLORADO STATE UNIVERSITY}

Because strong interactions between global change and resource management may determine whether the soil will serve as a source or sink for atmospheric carbon, the interactive effects of climate, elevated $\mathrm{CO}_{2}$, and management in the central United States will be separated by applying a global-change system analysis to a regional benchmark database. This separation will be done by (1) analyzing the effects of historical management practices at the regional sites; (2) projecting future effects of current management; (3) projecting the effects of current management with changed climate and $\mathrm{CO}_{2}$ levels; (4) optimizing management-system design for carbon storage and/or crop production; and (5) evaluating the relative impacts of management, $\mathrm{CO}_{2}$, and climate on carbon fluxes.

\section{CLIMATE CHANGE IN THE MIDCONTINENT OF NORTH AMERICA}

\author{
GOSNOLD, WILLIAM D., and \\ TODHUNTER, PAUL
}

\section{UNIVERSITY OF NORTH DAKOTA}

Recent analyses of the air-temperature records, specifically in the midcontinent of North America, suggest that no statistically significant trends are detectable and that at least 10 to 20 years of additional data collection may be necessary. However, analysis of existing geothermal gradient data collected during heatflow research in North Dakota, South Dakota, and Nebraska suggests that climatic warming of the order of 1.0 to $2.5^{\circ} \mathrm{C}$ has occurred in this region during the past century. The geothermal-gradient data show a systematic pattern of climate change in which the onset of warming appeared in the north and progressively advanced to the south. Thus, the geothermal-paleoclimate data show a pattern of warming in North America that agrees with the predictions of global-climate models based on increased concentrations of greenhouse gases; but, because of statistical uncertainty, the air-temperature analyses cannot yet detect the warming. Sufficient geothermal-paleoclimate data will be acquired to 
evaluate the climate history of the midcontinent and to comparatively analyze the geothermal-paleoclimate data and air-temperature data.

\section{EFFECT OF ECOSYSTEMS ON CLOUD MICROPHYSICS AND AEROSOL DISTRIBUTION}

\author{
HAN, QINGYUAN, and \\ WELCH, RONALD M.
}

\section{SOUTH DAKOTA SCHOOL OF MINES AND TECHNOLOGY}

A technique of retrieving effective droplet radii in liquid-water clouds on a global scale has been developed. The initial results show that Channel 3 of the AVHRR can be used for retrieving cloud droplet effective radius, that the difference in cloud droplet radii between continental-maritime clouds is a global phenomenon, and that the cloud microphysics over different ecosystems are systematically different. This research will be extended to establish (1) the relationships among cloud cover, cloud droplet size, aerosol distributions, and ecosysiems based on global-scale observations and (2) the relative importance among vegetation- and soiloriginated, anthropogenic, and ocean-biosphere-originated aerosols on cloud microphysics. Knowledge of these relationships will provide an estimate of the effect of human activities on cloud microphysics and will supply an observational basis for cloud modeling in GCMs.

\section{DETECTION OF CLIMATE CHANGE WITH LIVING AND EXTINCT DIATOM FLORAS}

\author{
HOAGLAND, KYLE D., and \\ ERNST, STEPHEN G.
}

\section{UNIVERSITY OF NEBRASKA-LINCOLN}

A method will be developed to use diatoms (Bacillariophyceae) as sensitive biological indicators of climate change past and present. A previous latitudinal gradient of diatom clonal isolates of Fragilaria capucina from Texas to South Dakota will be extended to include North Dakota and Manitoba. The new clones will be characterized physiologically and genetically with respect to their optimum growth temperature and thermaltolerance range. Taxonomic, cell-wall morphological features of clones from the entire gradient will be quantified to identify conservative, strain-specific characters. The diatoms from a lake-șediment core along the gradi- ent will be quantified. Specific hypotheses concerning climate change produced by the diatom assemblage will be tested against the historic climate record.

\section{SATELLITE OBSERVATION OF LAKE ICE AS A ROBUST INDICATOR OF REGIONAL CLIMATE CHANGE}

\author{
LILLESAND, THOMAS M., and \\ MAGNUSON, JOHN J.
}

\section{UNIVERSITY OF WISCONSIN}

Several investigators have suggested that the phenology of lake ice is a robust, integrated indicator of regional and global climate change. During 1990-1991, we employed satellite data from the NOAA AVHRR to study ice formation and breakup on the 45 lakes and reservoirs in Wisconsin having a surface area greater than $1000 \mathrm{ha}$. We found a very strong correlation $(R=-0.87)$ between the satellite-derived date of ice breakup and the mean January-March temperature at each lake location. Current and archival satellite data will now be used to typify the spatial and temporal distribution of spring lake-ice breakup from 1978 to 1994 along a central North American transect including the large lakes and reservoirs ( $>1000 \mathrm{ha}$ ) of Wisconsin, Northern Michigan, Minnesota, North and South Dakota, and portions of Manitoba and Ontario $\left(43^{\circ} \mathrm{N}\right.$ to $\left.57^{\circ} \mathrm{N}\right)$. The specific objectives of this work include (1) validation of the temporal and spatial extendibility of our earlier results, (2) spatially explirit retrospective modeling and trend analysis of lake-ice phenology throughout our study area, (3) prospective modeling of lake-ice response to alternative climate scenarios, and (4) further development of a climate-change indicator having particular applicability in regions where temperature measurements are sparse or nonexistent.

\section{DEVELOPMENT OF A NESTED REGIONAL MODEL FOR THE CONTERMINOUS UNITED STATES AND FORMATION OF HIGH- RESOLUTION CLIMATE-CHANGE SCENARIOS WITH AN APPLICATION TO CROP CLIMATE MODELS}

MEARNS, LINDA; GIORGI, F.; EASTERLING, W.; and WEISS, $A$.

\section{NATIONAL CENTER FOR ATMOSPHERIC RESEARCH and UNIVERSITY OF NEBRASKA-LINCOLN}


A regional climate-modeling framework will be developed that will lead to a detailed regional scenario of climate change over the conterminous United States. The climate-change scenario will be produced from a regional (mesoscale) climate model (RegCM) embedded in a GCM. The physics of the RegCM will be improved, different physics parameterizations and spatial resolutions will be tested by simulating the climate over the United States with a series of years of ECMWF boundary conditions, and the different runs will be carefully compared and validated. As part of the validation procedure, several crop-growth models will be applied to the simulated climate and will thus serve as an integrated validation of the $\mathrm{RegCM}$. A newer generation of GCM will be evaluated and used to drive the RegCM to form the climate-change scenario from the control and doubled- $\mathrm{CO}_{2}$ runs. Crop models will be run with RegCM output in all three RegCM simulations to serve as an additional integrated validation and to provide information regarding possible impact of the particular climate-change scenario on crop models.

\section{DETECTION OF CLIMATE CHANGE WITH LONG-TERM DAILY CLIMATE RECORDS OVER GRASSLAND REGIONS OF THE NORTHERN HEMISPHERE}

\section{PALECKI, MICHAEL; DEWEY, KENNETH F.; LEATHERS, DANIEL; and ROBINSON, DAVID \\ UNIVERSITY OF NEBRASKA-LINCOLN, UNIVERSITY OF DELAWARE, and RUTGERS UNIVERSITY}

New methods will be developed to aid the detection of human-induced climate change. These methods will use daily temperature, precipitation, snowfall, and snowcover data in an innovative manner to search for the low-frequency signal of cliniate change hidden in the structure of long, continuous, time series (up to 100 years). The temperate grasslands of the Northern Hemisphere will be the geographic focus of this research project; these regions are characterized by large annual cycles and steep spatial gradients of climate variables, providing the temporal and spatial sensitivities needed for detecting climate change. The analyses produced by this project will lead to improved understanding of the climate dynamics governing various aspects of observed climate changes.

\section{NATURAL RESPONSES OF SHALLOW LAKES AND WETLANDS FOR DETECTING CLIMATIC AND ENVIRONMENTAL CHANGE}

\author{
RUNDQUIST, DONALD C., and \\ GOSSELIN, DAVID C.
}

\section{UNIVERSITY OF NEBRASKA-LINCOLN}

The Nebraska Sand Hills, because of their geographic position within the Great Plains, landscape homogeneity, abundant water resources, and general environmental sensitivity, can serve as an important indicator region for assessing climate and environmental change. Certain environmentally induced spectral signals and fluxes emanating from and/or associated with the hydrological, biological, and microclimatological subsystems of selected Sand Hills wetlands will be identified, analyzed, and monitored after a baseline condition has been established for selected lakes and wetlands by analyzing historical remote-sensor data (including aerial photos dating back to the 1930s), groundwater levels, and climate information. Objectives include relating remote measurements of areal fluctuations in shallow lakes and wetlands to variations in groundwater levels, linking field studies of algal and macrophyte primary productivity to spectral data acquired both at close range and at satellite altitudes, and characterizing radiation fluxes and energy flows within wetland ecosystems and relating such measurements to remotely acquired spectral signals.

\section{ASSESSMENT OF CLIMATE- AND MANAGEMENT-INDUCED DIRECTIONAL CHANGES IN GREAT PLAINS VEGETATION WITH NDVI AND STABLE CARBON ISOTOPES}

\section{TIESZEN, LARRY L.}

\section{AUGUSTANA COLLEGE}

Carbon dioxide removed from the atmosphere by photosynthesis transmits a label to the pools of organic matter in natural systems that quantifies the amounts of $\mathrm{CO}_{2}$ sequestered by $\mathrm{C} 3$ and $\mathrm{C} 4$ photosynthetic types. Because the distributions of these types and their biomass production are functions of growing-season temperature, they provide a potentially powerful tool to assess climate change and the steady-state relationships between ecosystem properties and atmospheric trace gases. Applications of stable-isotope approaches will 
address ecosystem change in response to climate, the development of a paleothermometer, an assessment of $\mathrm{CO}_{2}$ pools and fluxes in the Great Plains, and the role of land management and remediation. Major grasslands will be studied across broad latitudes to document Holocene climate changes in the Great Plains, to assess the departure of soil-organic-matter pools from steady state, to quantify the contributions of some systems to regional $\mathrm{CO}_{2}$ fluxes, and to provide regional maps of these functional features and anticipated responses to future climate changes and management systems.

\section{AN INTEGRATED INVESTIGATION OF METHANE AND CARBON DIOXIDE FLUXES IN MIDLATITUDE PRAIRIE WETLANDS: MICROMETEOROLOGICAL MEASUREMENTS, PROCESS-LEVEL STUDIES, AND MODELING}

VERMA, SHASHI B.

UNIVERSITY OF NEBRASKA-LINCOLN
Spatially integrated exchange rates of $\mathrm{CH}_{4}$ and $\mathrm{CO}_{2}$ will be quantified at selected midlatitude prairie wetland sites over the course of a growing season with the micrometeorological eddy-correlation technique. Variability in $\mathrm{CH}_{4}$ and $\mathrm{CO}_{2}$ fluxes within and across selected wetlands in the region will be evaluated with chambers. Factors limiting $\mathrm{CH}_{4}$ production and oxidation will be quantified with process studies (field experimental manipulations), and responses of leaf photosynthesis, respiration, and stomatal conductance of dominant plant species to relevant controlling variables will be experimentally quantified. These components will be integrated to test and improve models of $\mathrm{CH}_{4}$ and $\mathrm{CO}_{2}$ exchanges, and the field measurements will allow temporal and spatial variation in fluxes of $\mathrm{CH}_{4}$ and $\mathrm{CO}_{2}$ to be related to underlying biological and biophysical processes.

\section{Midwestern Regional Center}

\section{DROP-SIZE-DEPENDENT NATURE OF CLOUD CHEMISTRY AND ITS RELATIONSHIP TO THE PROCESSING OF ATMOSPHERIC AEROSOLS AND TRACE GASES}

\section{COLLETT, JEFFREY L.}

\section{UNIVERSITY OF ILLINOIS}

The extent of chemical heterogeneity present among cloud drops formed in a variety of environments with different cloud types and pollutant levels will be determined. Both backgtound and anthropogenically influenced sites will be used to reveal the effects of human activities, particularly emission of sulfur dioxide and other potential $\mathrm{CCN}$-forming species, on these processes. Drop-size-fractionated cloud samples will be collected with a multistage jet impactor.

Samples will be analyzed to determine how major ion concentrations and acidity vary with drop size. This information will be combined with drop-size-dependent measurements of sulfur and oxidant species to determine how the rate of sulfur oxidation varies with drop size. The dependence of aerosol composition on particle size will he used in conjunction with the dependence of ion concentrations on cloud-drop size to provide insight into which aerosol sizes are removed most efficiently through processes of accretional, precipitative growth. The fate of accumulation-mode aerosol particles, including sulfates, is particularly important because of their dual role as efficient radiation scatterers and as CCN.

\section{ANALYSIS OF REGIONAL ECONOMIC AND ENERGY-DEMAND IMPACTS ARISING FROM GLOBAL ENVIRONMENTAL CHANGE}

$$
\text { RUBIN, BARRY M. }
$$

\section{INDIANA UNIVERSITY}

An integrated economic-environmental-energyconsumption model that can be applied at a regional watershed level will be designed to translate climatechange effects data on groundwater flow, surface hydrology, biological productivity, and wetland ecology into impacts on economic activity. The model will also predict the direct impact of regional climate variation on energy' consumption of households, the private economy, 
and the public sector. The modeling effort will initially be applied to the four-county region encompassing the Pere Marquette Watershed of Central Michigan.

Preliminary results for the Pere Marquette Watershed indicate that definitive, climate-induced economic impacts exist and can be identified through the integrated, econometric economic-ecologic-energydemand model. These initial results as well as the determination of structural variations among climaterelated impacts in rural vs urban regional economies will be verified. To generalize the modeling framework for forecasting climate-change impacts, an urban watershed region (the five-county Milwaukee Consolidated Metropolitan Area in southeastern Wisconsin) will be modeled. This area is highly urbanized, is adjacent to Lake Michigan, and has an economic base typical of many other industralized areas in the midwest and northeast. The availability of economic and environmental data for this area also provides the potential for disaggregating of the model to allow for a larger number of economic sectors and for a detailed quarterly analysis of ecological, economic, and energy data. This latter component of the analysis will also allow for specifically incorporating seasonal variations related to climate change.

\section{TRACE-GAS FLUXES IN AGRICULTURAL ECOSYSTEMS: BIOSPHERIC FEEDBACKS}

$$
\text { ROBERTSON, G. PHILIP }
$$

\section{MICHIGAN STATE UNIVERSITY}

The importance of major midwest U.S. cropping systems to increases in the atmospheric concentrations of $\mathrm{CO}_{2}, \mathrm{CH}_{4}$, and $\mathrm{N}_{2} \mathrm{O}$ will be assessed with replicated cropping systems representing major row-crop ecosystems of the U.S. Midwest. Both extensive sampling of eight systems on an event-driven basis and an intensive, near-continuous measurement program will be carried out with three annual and one perennial cropping systems.

The work will have three specific objectives: (1) to examine the predictability of short-term changes in $\mathrm{N}_{2} \mathrm{O}$ production and $\mathrm{CH}_{4}$ uptake in different cropping systems and determine whether these fluxes integrate to regionally significant values; (2) to examine the extent to which $\mathrm{CO}_{2}$ fluxes from agricultural soils are related to soil nitrogen status and the size of the active fraction of soil organic matter; and (3) to examine the causal factors regulating the temporal dynamics of $\mathrm{CH}_{4}$ and $\mathrm{N}_{2} \mathrm{O}$ fluxes in agricultural systems. This information will be used to further develop, parameterize, and validate mechanistic models of these trace-gas fluxes from agricultural soils.

\section{CONTRASTING SUCCESSIONAL RATES AND CARBON CYCLING IN EUTROPHIC AND OLIGOTROPHIC ECOSYSTEMS OF AMAZONIA}

\author{
MORAN, EMILIO F. \\ INDIANA UNIVERSITY
}

A comparative analysis will be made of deforestation and ecological succession in the Brazilian Amazon, and the role of these processes in the global carbon cycle will be examined. Two oligotrophic sites in the Zona Bragantina will be contrasted to two eutrophic sites (one in the Lower Xingu basin and the other at Marajo Island). Data will be collected on history of land use; estimates of the biomass standing crop; carbon-content and nutrient characterization $(\mathrm{N}, \mathrm{P}, \mathrm{K}, \mathrm{Ca}, \mathrm{Mg})$ of plants, litter, and soils; and determination of soil $\mathrm{pH}$, cation-exchange capacity, and texture. These field data will be integrated with remotely sensed imagery of these regions with both vector- and raster-based geographic information systems (GISs). An important component in this analysis is the joint use of multitemporal Landsat Thematic Mapper and ERS-1 radar digital data to enhance the terrain analysis. A carbon-balance model will be developed with field data and geo-referenced to specific sites with a GIS. Land-use and carbon-balance models will be used to compare rates of deforestation and ecological succession in the eutrophic and oligotrophic forest ecosystems. The results will be used to examine the role of tropical-forest deforestation and reforestation (succession) in the Amazon Basin in the global carbon cycle.

MODELING THE IMPACT OF ECONOMIC TRANSITION IN EASTERN-BLOC COUNTRIES ON $\mathrm{CO}_{2}$-EMISSION PROFILES: A DYNAMIC ENVIRONMENTAL-ECONOMIC SIMULATION

\section{KRUTILLA, KERRY M. \\ INDIANA UNIVERSITY}

Eastern-Bloc countries, which jointly contribute $24 \%$ of global $\mathrm{CO}_{2}$ emissions, are undergoing a transition to 
market-oriented systems that will produce significant changes in $\mathrm{CO}_{2}$ emissions. $A$ dynamic economicenvironmental model will be developed that integrates structural change, technological progress, and $\mathrm{CO}_{2}$ abatement policy and incorporates the unique features of dynamic economic transition. The model will be applied to a case study on Russia, the largest contributor to regional $\mathrm{CO}_{2}$ output, to produce $\mathrm{CO}_{2}$-emission profiles for plausible economic transition scenarios from the present to the year 2050, projected abatement costs, and cost-benefit estimates on the efficacy of Western technical and financial assistance.

\section{CLIMATE-CHANGE EFFECTS ON AGRICULTURE IN THE GREAT LAKES REGION}

LEE, JAE K.

\section{INDIANA UNIVERSITY}

Because $\mathrm{CO}_{2}$-induced climate change will cause changes in the pattern and potential production of world agricultural regions, the sensitivity of the Great Lakes agricultural systems to future climatic change will be studied. Various climatic and hydrologic factors controlling agricultural production will be evaluated to assess agricultural suitability and potential productivity over the study region. A relationship between the predicted potential productivity and actual distribution and production rates will be identified. The GCMderived $2 \times \mathrm{CO}_{2}$ climate scenarios will be applied to the potential productivity of agricultural types to assess the sensitivity of crop potential to future climate change and to provide information for refining crop-yield models and developing adaptation strategies for future changes in U.S. agriculture.

\section{ABOVE- AND BELOW-GROUND ECOSYSTEM RESPONSES TO ELEVATED ATMOSPHERIC $\mathrm{CO}_{2}$}

TEERI, JAMES A.

\section{UNIVERSITY OF MICHIGAN BIOLOGICAL STATION}

To understand how rising atmospheric $\mathrm{CO}_{2}$ will alter the cycling of carbon from roots and mycorrhizae to soil microorganisms and how this will in turn feed back on nitrogen availability and plant productivity, long-term experiments will be carried out with elevated $\mathrm{CO}_{2}$ and poplar trees grown in open-topped chambers. Fine-root dynamics and soil-arthropod activity will be quantified in situ with advanced video technology and computerassisted image analysis. Labelled nitrogen will be used to measure nitrogen cycling under elevated- and ambient- $\mathrm{CO}_{2}$ concentrations. Whole-tree net $\mathrm{CO}_{2}$ uptake is expected to show a sustained increase under elevated atmospheric $\mathrm{CO}_{2}$, and roots and mycorrhizae are expected to play a key role in preventing negative acclimation. Increased fine root growth, root turnover, micro-anthropod activity, and mycorrhizal infection are expected to result in increased microbial biomass and nitrogen availability.

\section{AN INVESTIGATION OF THE PHYSICAL AND BIOGEOCHEMICAL PROCESSES CONTROLLING METHANE EMISSIONS FROM PEATLAND ECOSYSTEMS}

\author{
WHITE, JEFFREY R.
}

\section{INDIANA UNIVERSITY}

To reduce the uncertainties in estimates of spatial and temporal patterns of $\mathrm{CH}_{4}$ emissions from peatlands, fieldwork has been conducted on the physical and biogeochemical controls of $\mathrm{CH}_{4}$ production, consumption, and exchange with the atmosphere in peatland ecosystems. In this fieldwork, we have observed strong seasonal variations (100-fold differences) in net $\mathrm{CH}_{4}$ flux rates at each of our sites. We have identified a complex set of interactions involving water levels, peat temperatures, and direct emission by emergent plants that control rates of $\mathrm{CH}_{4}$ emissions. These findings will be verified with field measurements, and a series of soil-incubation experiments and peat-core experiments designed to isolate the effects of substrate competition, water level, and soil temperature on $\mathrm{CH}_{4}$ production and consumption in peat soils will be conducted.

\section{PREDICTING THE BASINWIDE RESPONSES OF INTERIOR WETLANDS TO GLOBAL CLIMATE CHANGE}

$$
\text { PARK, RICHARD }
$$

\section{INDIANA UNIVERSITY}

An integrative approach that combines remote sensing, GISs, and simulation modeling will be developed and tested to predict the impacts of global climate change on 
the extent and types of wetlands over large geographic areas. Changes in production of $\mathrm{CO}_{2}$ will be estimated, and the effects of human disturbances that might exacerbate changes in wetlands will be assessed. The distribution of major classes of wetlands will be mapped for an entire watershed comprising a landscape mosaic typical of Midwestern glaciated terrain. The responses of the wetlands to climate-induced changes in regional, subregional, and local water tables (including the effects of increased irrigation) will be simulated. The results will be summarized in terms of increased contribution to the pool of atmospheric $\mathrm{CO}_{2}$.

\section{CLIMATE FORCING BY AEROSOL PARTICLES IN THE MIDWESTERN UNITED STATES}

ROOD, MARK J.

\section{UNIVERSITY OF ILLINOIS}

To investigate the direct effects of aerosols on the globe's energy balance, the mass-scattering crosssections of ambient aerosols and the variability of those cross-sections will be measured at midwestern continental sites in the U.S. These measurements will be achieved by construction of aerosol-sampling instrumentation, operation of the instrumentation at a well characterized field site for 1.75 years, and interpretation of the results. Results obuined at the site will be compared with results from previously developed thermodynamic and light-scattering models.

\section{EFFECT OF ATMOSPHERIC AEROSOLS ON OPTICAL AND RADIATIVE PROPERTIES OF THE ATMOSPHERE}

$$
\text { LARSON, SUSAN M. }
$$

\section{UNIVERSITY OF ILLINOIS}

Numerical modeling and experimental work will be pursued to link the chemical and physical characteristics of atmospheric aerosols with atmospheric optical properties. At rural and urban midwestern locations, field measurements will be taken of the composition, concentration, and particle-size distribution of ambient suspended particulate matter and the concentration of major pollutant gases. The atmospheric light-scattering coefficient, solar radiation, and weather parameters will also be determined. The modeling effort will examine aerosol and light-extinction models predicting the size, water content, and refractive index of aerosol particles, as well as surface irradiation, light scattering, absorption, and extinction efficiencies. The experimental data will be used as inputs to the numerical models to predict properties of atmospheric aerosol and radiation. Predicted values will be compared to measured parameters to verify the numerical models.

\section{AMAZONIAN DEFORESTATION, SUCCESSION, AND CARBON CYCLING: III. A GIS/SPECTRAL MULTITEMPORAL ANALYTICAL APPROACH}

\author{
MAUSEL, PAUL
}

\section{INDIANA STATE UNIVERSITY}

To examine the processes of forest succession in Amazonia, the 1985-1991 Landsat Thematic Mapper (TM) satellite data were obtained, a series of remotesensing procedures was tested that permitted the optimal identification of multitemporal spectral responses of features of interest, data collection was carried out in the field, and the classification of the vegetation classes was refined to incorporate the field data and to add another Landsat TM scene coincident with the fieldwork. These procedures allowed the development of precise spectral signatures for nine classes, three of which were secondary-successional stages. Ten to fifteen years of secondary succession spectrally approximated the signature for mature moist forest and resembled it in both biomass and architecture in the field.

The five dates of classified TM data will be integrated with the extended botanical, soil-topographical, and road-network data collected with GIS raster and vector approaches. This integration will permit evaluation of the rate and/or sequence of succession as a function of individual and combined parameters. ERS-1 digital radar data will be incorporated to improve land-cover and topographical results, and neural-network classification algorithms will be evaluated for the analysis of this type of study. 


\section{Northeast Regional Center}

\section{GENERALIZING THE CORE EXPERIMENT WORK AT HARVARD FOREST TO ADDRESS GLOBAL-CHANGE ISSUES THROUGH MODELING OF FOREST METABOLISM AND ANALYSIS OF SATELLITE IMAGERY}

\author{
AMTHOR, J. S., and DAVIDSON, E. A. \\ WOODS HOLE RESEARCH CENTER
}

An existing physiologically based model of forest ecosystem metabolism (i.e., carbon, water, and energy exchange) and carbon storage will be extended to include soil respiration and parameterized for Harvard Forest. The model is composed of modules simulating photosynthesis, photorespiration, stomatal conductance, canopy energy exchange, respiration, photosynthate partitioning, growth, fine-root turnover, and litter production. It will be tested with data being collected in the core experiments at Harvard Forest. The goal will be to generalize results of the Harvard Forest experiments and to predict the effects of increasing atmospheric $\mathrm{CO}_{2}$ and climate change on forest carbon -ycling and storage.

The seasonal course of the normalized-difference vegetation index (NDVI) will be derived at several spatial scales from 1-km NOAA AVHRR satellite images of Harvard Forest and will be compared with $\mathrm{CO}_{2}$ fluxes measured at the Harvard Forest instrument tower. Derived relationships between NDVI and ecosystem $\mathrm{CO}_{2}$ flux will be used to explore the feasibility of spatially extrapolating Harvard Forest measurements.

\section{PREDICTING RESPONSES OF TEMPERATE- FOREST ECOSYSTEMS TO ELEVATED ATMOSPHERIC-CO ${ }_{2}$ CONCEN'TRATIONS AND AIR TEMPERATURES}

BAZZAZ, F. A.

\section{HARVARD UNIVERSITY}

The current capability to predict the potential effects of global change on forest ecosystems will be improved by studying the responses of seedlings and saplings of 14 important New England temperate-forest species to six combinations of atmospheric- $\mathrm{CO}_{2}$ concentrations and air temperatures over a period of 3 years. Leaf-level carbon flux will be measured from plants grown in controlled environments, and in conjunction with measurements made on mature trees in Harvard Forest, changes in forest-ecosystem carbon uptake in response to predicted global change scenarios will be analyzed. Wholeseedling and -sapling responses will be used to make predictions regarding species' differential regeneration patterns in elevated $\mathrm{CO}_{2}$ and temperature environments, and the longer-term consequences of changes in species composition to northeastern temperate forest ecosystems will be assessed.

\section{COMPOSITION OF AIR IN THE FIRN LAYER OF ICE SHEETS AND THE RECONSTRUC- TION OF ANTHROPOGENIC CHANGES IN GREENHOUSE-GAS CONCENTRATIONS}

\author{
BENDER, M. A. \\ UNIVERSITY OF RHODE ISLAND
}

The concenuation and isotopic composition of greenhouse gases and the $\mathrm{O}_{2} / \mathrm{N}_{2}$ ratio of air in the firn of ice sheets will be studied. (The firn is the upper 50-to-100$\mathrm{m}$-thick layer of incompletely compacted snow.)

The studies will elucidate how diffusion and convection influence the composition of air in the firn, especially at its base, where air is trapped in bubbles in the ice. That information will allow refining reconstructions of how the concentration and (especially) the isotopic composition of greenhouse gases in air changed because of man's activities.

The studies will also measure the increase with depth in the $\mathrm{O}_{2} / \mathrm{N}_{2}$ ratio of air in firn at several different sites. These data will allow calculating the total rate of anthropogenic $\mathrm{O}_{2}$ consumption by burning fossil fuels and by converting biomass to $\mathrm{CO}_{2}$ during the past two to three decades. From this rate of $\mathrm{O}_{2}$ consumption and the rate of increase in the $\mathrm{CO}_{2}$ concentration of air, the net growth rate of the land biosphere and the dissolution rate of anthropogenic $\mathrm{CO}_{2}$ in the oceans will be calculated. 


\section{BIOLOGICAL AND PHYSICAL CONTROLS ON SOIL-ORGANIC-MATTER QUALITY AND QUANTITY}

\author{
BOONE, R. D., and NADELHOFFER, K. J. \\ HARVARD UNIVERSITY and MARINE \\ BIOLOGICAL LABORATORY, WOODS HOLE
}

Long-term litter-manipulation plots were established in a mid-aged, mixed-hardwood stand at the Harvard Forest from 1990 to 1991 to evaluate biological and physical controls on soil organic matter (SOM) storage. Assessing these controls is critical because soils contain a large portion of terrestrial carbon, carbon fluxes between soils and the atmosphere are large, and the factors controlling soil carbon storage are inadequately understood. Litter manipulations on the Harvard Forest plots are simple, but will provide fundamental information on (1) the relative contributions of roots and foliar litter to total SOM and to active and stabilized fractions, (2) the partitioning of total soil $\mathrm{CO}_{2}$ efflux among root respiration and the decomposition of different organic-matter pools, (3) the recovery patterns for total SOM and its component kinetic fractions after soil impoverishment, (4) the potential of this typical New England soil to sequester carbon, and (5) the effect of changes in SOM on nutrient content and availability.

\section{MODERN CALIBRATION OF DEEP-OCEAN CLIMATE RECORDERS: MULTIPLE-TRACER STUDIES OF LIVING BENTHIC FORAMINIFERA}

\author{
BOYLE, E. A.
}

\section{MASSACHUSETTS INSTITUTE OF TECHNOLOGY}

Oceanographers studying deep-ocean climate and circulation changes rely on geochemical tracers incorporated into benthic foraminifera. While there is agreement that foraminifera generally record these properties with sufficient accuracy to establish the largescale large-amplitude signals, it is still uncertain if foraminifera are sufficiently precise to record more subtle but important signals. Core-top calibrations of fossil benthic foraminifera cannot resolve this question because any mismatches between core-top benthic foraminifera and modern bottom water are as likely to be caused by flaws in quality of core tops (because of bioturbation and other forms of reworking) as by the ability of foraminifera to record bottom-water properties. The ultimate test of accuracy and precision is a comparison between scarce living benthic foraminifera and modern bottom water. A strategy will be implemented for obtaining the large samples of living benthic foraminifera required for the calibration of multiple geochemical tracers.

\section{ROLE OF BAROCLINIC-WAVE AMPLITUDE AND TRANSPORT VARIA'IION IN CLIMATE CHANGE}

FARRELL, B. F.

\section{HARVARD UNIVERSITY}

The primary dynamical agents of heat and moisture transport in midlatitudes are baroclinic waves of synoptic and planetary scale. Recent developments in the theory of non-selfadjoint systems have made possible a physically based understanding of the sensitivity of baroclinic-wave amplitude and phase to modification of atmospheric latent- and sensible-heat distribution, such as might be associated with changes in concentration of radiatively active atmospheric gases. Analysis of a realistic, baroclinic, zonal jet model with adjoint techniques revealed that the sensitivity of baroclinic-wave response arises from rapid growth mediated by induced transfer of mean-flow potential energy to the wave scale. The sensitivity of the forced response of planetary-scale baroclinic waves responsible for much of observed regional climate variability will now be determined. A generalizable mechanism will be provided for determining the sensitivity of baroclinic waves at synoptic and planetary scales to climate forcing, and it will be applicable to the analysis of results from any GCM for which an adjoint is available.

\section{OBSERVATION, ANALYSIS, AND PARAMETERIZATION OF FOREST- ATMOSPHERE EXCHANGE MECHANISMS}

\author{
FITZJARRALD, D. R., and MOORE, K. E. \\ STATE UNIVERSITY OF NEW YORK AT \\ ALBANY
}

A turbulence climatology will be constructed of the seasonal interaction between a deciduous forest and the atmosphere. The turbulence-observation network, tracking the annual course of the heat, momentum, water vapor, and radiation budgets at the forest top, will 
be maintained. An analysis of strategy will be carried out based on composting forest response (heat and water vapor fluxes and $\mathrm{CO}_{2}$ uptake) to synoptic disturbances according to seasons; developing parameterizations of turbulent transports based on identification of fluxes with coherent eddies above the forest and finding their efficacy in removing trace gases from the forest floor; and using the observations to test and refine parameterization of forest-atmosphere exchange currently used in large- and regional-scale climatechange models.

\section{SOURCES OF ATMOSPHERIC METHANE IN THE EASTERN UNITED STATES}

\section{HARRISS, R. C., and CRILL, P. \\ UNIVERSITY OF NEW HAMPSHIRE}

The data record from the first year of $\mathrm{CH}_{4}$ measurements at the NIGEC tower in Harvard Forest documented numerous episodes of enhanced $\mathrm{CH}_{4}$ concentrations. Critical measurements will be obtained to fill gaps in the data. A detailed regional $\mathrm{CH}_{4}$ source inventory will also be assembled in a GIS to link sources, transport, and measured concentrations. The emissions inventory will include the first quantitative estimates for wastewater treatment systems and landfills, which are probably the major sources of $\mathrm{CH}_{4}$ pollution in the northeastern U.S. The combination of $\mathrm{CH}_{4}, \mathrm{NO}_{y}$, $\mathrm{NO}_{\mathrm{x}}, \mathrm{CO}, \mathrm{CO}_{2}$, and other parameters will enable development of source-area chemical fingerprints. The results of this investigation will provide a sound basis for planning a U.S. national strategy for mitigation and monitoring of continental sources of greenhouse gases.

\section{DYNAMIC HEAT AND MOISTURE TRANSPORT AND BAROCLINIC ADJUSTMENT}

LINDZEN, R. S.

\section{MASSACHUSETTS INSTITUTE OF TECHNOLOGY}

Basic processes in atmospheric dynamics relevant to climate will be studied, including nonlinear baroclinic equilibration. Whether a newly discovered neutral state is realistic will be determined. Preliminary observational analyses suggest it is, allowing calculations of meridional heat transport between the tropics and high latitudes. Cumulonimbus convection and the Hadley circulation dominate tropical transports. Cumulus convection, however, is highly localized so that the environment for such convection differs from the average tropical environment. Models for relating the cumulus environment to the mean tropical environment, and for interfacing the Hadley regime and the baroclinic eddy regime will be developed, with particular attention to improving understanding of the Hadley circulation and the mean thermodynamic properties of the tropics, including upper-level water vapor (which provides a crucial climate feedback). Finally, a relatively simple mechanistic climate model will be, developed incorporating the dynamical mechanisms and the equations for radiative transfer to calculate the zonally averaged, seasonally varying climate.

\section{ISOTOPIC COMPOSITION OF ATMOSPHERIC $\mathrm{CO}_{2}$ INFERRED FROM CARBON IN C4 PLANT CELLULOSE: STUDIES OF THE GLOBAL CARBON CYCLE}

MCELROY, M. B., and MARINO, B. D.

\section{HARVARD UNIVERSITY}

The ${ }^{13} \mathrm{C} /{ }^{12} \mathrm{C}$ ratios of carbon in selected plants with the C4 photosynthetic mode will be used to infer the isotopic composition of atmospheric $\mathrm{CO}_{2}$ in the past. Specifically, we plan to document changes in the isotopic composition of atmospheric $\mathrm{CO}_{2}$ from the present to about 40,000 years BP, emphasizing the historic, preindustrial, and Holocene periods. Preliminary data and model results for carbon-isotope ratios of $\mathrm{CO}_{2}$ for the modern, historic, and glacial to interglacial periods indicate new and potentially important information on the function of the global carbon cycle in the past. These studies will be extended with further investigations, both experimental and theoretical.

\section{ATMOSPHERE-BIOSPHERE FEEDBACK MECHANISMS IN FOREST ECOSYSTEMS}

\author{
MELILLO, J. M.; NADELHOFFER, K. J.; and \\ STEUDLER, P. A.
}

\section{MARINE BIOLOGICAL LABORATORY, WOODS HOLE}

Four questions will be addressed by analysis of changes in growth patterns and soil carbon and nutrient dynamics in a soil warming experiment at Harvard 
Forest, by analysis of harvested material fom trees grown for several years with elevated and normal levels of $\mathrm{CO}_{2}$, and by analysis of data from forest plots treated with inorganic nitrogen. How will an increase in forest soil temperature affect the fluxes of $\mathrm{CO}_{2}, \mathrm{CH}_{4}$, and $\mathrm{N}_{2} \mathrm{O}$ between the land and the atmosphere? Will elevated $\mathrm{CO}_{2}$ result in the production of plant-litter materials with altered carbon and nitrogen chemistries, and will these changes in litter chemistry slow the rate of litter decay? Will plants exposed to elevated $\mathrm{CO}_{2}$ allocate excess carbon to the rhizosphere to acquire growthlimiting nutrients, such as nitrogen? And what is the fate of nitrogen that is added to forest ecosystems, and what does this fate imply for ecosystem-wide carbon storage?

\section{INFLUENCE OF REGIONAL-SCALE TRANSPORT ON THE CONCENTRATION OF CLIMATICALLY IMPORTANT TRACE GASES AT HARVARD FOREST}

\author{
MOODY, J. L., and JACOB, D.
}

\section{HARVARD UNIVERSITY}

Three-dimensional back trajectories will be constructed for air parcels advected to the Harvard Forest airchemistry-monitoring site. With the model wind and temperature fields of the National Meteorological Center Nested Grid Model, four trajectories per day will be used to determine the patterns in regional-scale transport and their role in regulating the concentrations of climatically important trace gases (methane and nonmethane hydrocarbons) measured at the site. From the trajectories, distinct patterns of regional-scale transport to the Harvard Forest site and their relative frequency of occurrence will be identified, the highfrequency temporal variance of the chemical data will be interpreted in terms of chemical-source regions, chemical signatures and trace-gas ratios will be defined for the different air masses advected to the site, and the role of transport in determining the seasonal and interannual variations in trace-gas concentrations and fluxes will be assessed.

\section{SOURCE OF RADIATIVE EFFECT OF UPPER- LEVEL WATER VAPOR}

SMITH, R. B.

YALE UNIVERSITY
Results from our Cloud Isotope Pilot Project will be analyzed to improve our understanding of uppertroposphere water and its role in climate. A new field project will be carried out to collect samples of ice and vapor from convective cloud anvils and to analyze samples for deuterium isotopes. These data will help answer fundamental questions about how clouds carry water into the upper troposphere. A simple 1-D cloudphysics model will be developed to aid interpretation of the new isotope data. In situ measurements of upperlevel water vapor and the comparison with outgoing longwave radiation in the water-vapor vibration bands will be continued.

\section{COUPLED ATMOSPHERE-OCEAN MODELS}

\author{
STONE, P. H., and MAROTZKE, J. \\ MASSACHUSETTS INSTITUTE OF \\ TECHNOLOGY
}

Coupled atmosphere-ocean process models will be developed, intended to be simpler than coupled GCMs but to include a wider range of atmosphere-ocean interactions than earlier process models. The most sophisticated model will couple Marotzke and Willebrand's 3-D ocean GCM with an idealized geometry to Stone and Yao's 2-D statistical-dynamical model of the atmosphere. Sensitivity studies will be carried out with models designed to elucidate the feedbacks between the atmosphere and the oceanic thermohaline circulation, to identify the processes that are important in determining climate sensitivity, and to give guidance for improving the poor simulations of heat balance in current coupled GCMs.

\section{ATMOSPHERE-BIOSPHERE EXCHANGE OF NITRIC ACID IN A FOREST SETTING}

TALBOT, R. W.

\section{UNIVERSITY OF NEW HAMPSHIRE}

The dry deposition of atmospheric $\mathrm{HNO}_{3}$ (potentially the principal source of atmospheric nitrogen inputs to northern U.S. ecosystems) will be investigated at Harvard Forest. Data for $\mathrm{HNO}_{3}$ vapor and particulate nitrate will be obtained over a suite of diurnal and seasonal conditions to assess inputs of atmospheric nitrate to this northern forest ecosystem. Initial results show significant inputs of $\mathrm{HNO}_{3}$ vapor to the canopy that may be equal to or greater than wet deposition of 
nitrate. These ongoing measurements provide new insight on an important aspect of atmospheric-nitrogen inputs to northern U.S. forest ecosystems and help synthesize the measurements of $\mathrm{NO}_{x}$ and total reactive nitrogen $\mathrm{NO}_{\mathrm{y}}$ at Harvard Forest. This investigation also contributes to regional-to-global-scale assessments of ecosystem response to climate change and alteration of biogeochemical cycles.

\section{SCHUMANN-RESONANCE MEASUREMENTS AS A SENSITIVE DIAGNOSTIC FOR GLOBAL CHANGE}

\author{
WILLIAMS, E. R.
}

\section{MASSACHUSETTS INSTTTUTE OF TECHNOLOGY}

Calibrated measurements of Schumann resonance (SR), a global electromagnetic phenomenon driven by lightning, will be undertaken to provide a sensitive diagnostic for changes in global tropical surface temperature. The determination of lightning-source characteristics on a regional basis will be attempted with single-station Schumann measurements. The source characteristics of major zones of deep tropical convective activity will be compared with surface measurements of wet-bulb temperature, the parameter that largely determines the degree of conditional instability in the tropics in the current climate. Particular attention is to be given to the response of SR to tropical seasonal temperature variations, which are of the order of $1^{\circ} \mathrm{C}$.

\section{REDUCING EMISSIONS OF GREENHOUSE GASES BY ALTERNATIVE ENERGY SOURCES}

\author{
WILSON, R, and KAMMEN, D. M.

\section{HARVARD UNIVERSITY}

Alternative energy technologies, frequently touted as ideal vehicles to drive economic growth and development while limiting the emission of radiatively active trace gases, have demonstrated a slow, and sometimes stagnant, history of technology diffusion and rate of market penetration. The mix of technological and policy issues that have contributed to this stagnation will be examined in detail for two alternative-energy sectors: nuclear poiver and solar thermal systems. The dynamics of these sectors will be explored with these qualitatively distinct models: a carbon-tax scenario, a macroeconomic-growth model, and a new dynamical-systems approach. The technological and social policy options available to manipulate the use of these industries promise to have application to a range of alternativeenergy systems.

\section{DIRECT MEASUREMENTS OF CARBON DIOXIDE AND NITROGEN OXIDE FLUXES IN CENTRAL NEW ENGLAND}

\author{
WOFSY, S. C.; GOULDEN, M. L.; MUNGER, J. W.; \\ and DAUBE, B. C.
}

\section{HARVARD UNIVERSITY}

Net ecosystem exchange of $\mathrm{CO}_{2}$ was measured in a transition hardwood forest in central Massachusetts with the eddy-correlation method. Ecosystem respiration rates were similar to predictions of global models, but carbon fixation was more efficient than expected, $\sim 0.028$ molecule/photon. Net uptake of $\mathrm{CO}_{2}$ exceeded 4 tonnes $\mathrm{C} / \mathrm{ha}$ each year. Carbon uptake by temperate forests appears to have been underestimated because of difficulty in quantifying storage below ground. Temperate forests can potentially accumulate globally significant quantities of carbon and represent a significant factor in regulating concentrations of atmospheric $\mathrm{CO}_{2}$.

Wet and dry deposition of $\mathrm{NO}_{\mathrm{y}}$ were measured with eddy correlation to determine dry deposition. Total deposition reflected approximate balance between regional emissions and deposition. The fraction of deposition wet vs dry was highly variable, regulated by atmospheric oxidation and scavenging rates.

Concentrations and fluxes of C2-C8 hydrocarbons were measured for one year, providing information on fluxes of isoprene and on concentrations and sources of important species, such as ethane, propane, and acetylene. 


\section{Southeast Regional Center}

\section{CARBON BALANCE AND GROWTH ADAPTATION OF CONTRASTING $\mathrm{C}_{3}$ AND $\mathrm{C}_{4}$ PERENNIAL FORAGE SPECIES TO INCREASED $\mathrm{CO}_{2}$ AND TEMPERATURE}

BOOTE, K. J.

\author{
UNIVERSITY OF FLORIDA
}

The carbon-balance dynamics of perennial forage species under projected increases in $\mathrm{CO}_{2}$ and temperature will be studied to understand the degree to which these species act as sources and sinks for $\mathrm{CO}_{2}$ and to evaluate feedback effects of climate change on productivity and carbon-sink capacities of these plants. Perennial forages are important because in rotations they sustain productivity and because they commonly replace cut forests. The two study species are long-lived perennials that sequester considerable carbon in underground rhizomes or surface-level stolons. Bahiagrass (Paspalum notatum) is a $\mathrm{C}_{4}$ pasture grass that predominates on large acreages in the southeastern United States, while perennial peanut (Arachis glabrata) is a long-lived $C_{3}$ forage legume. The two species will be grown under two $\mathrm{CO}_{2}$ levels and at two temperatures in temperature-gradient greenhouse tunnels. The experiment will evaluate the rate of establishment; growth processes; herbage nutritive value; and carbonsequestering rhizomes, roots, and shoots.

\section{ESTIMATION OF GROUND-SURFACE TEMPERATURE FROM BOREHOLE TEMPERATURE DATA}

\section{BRETT, C. EVERETT}

\section{UNIVERSITY OF ALABAMA}

The temperature profile in the Earth's crust is governed by the ambient ground-surface temperature and by the Earth's geothermal gradient. For areas in which tectonic or other activities are nonexistent, the temperature profile is piecewise linear, with discontinuities in the gradient occurring at stratigraphic boundaries where changes occur in the thermal properties of the crust. Anomalous curvature of the temperature profile may be caused by transients in the Earth's surface temperature. Because of the time required for surface-temperature variations to propagate downward into the crust, the temperature profile in the crust exhibits a memory of the past surface-temperature history. Measurements of the temperature profile in the crust may be used to deduce the nature of that history, thus providing direct measurement of possible human-induced climate change.

\section{CONTROL OF METHANE FLUX FROM VEGETATED WETLANDS}

\section{CHANTON, JEFFREY}

\section{FLORIDA STATE UNIVERSITY}

Wetlands, both natural and agricultural systems, contribute an estimated $40 \%$ of the total $\mathrm{CH}_{4}$ emitted to the atmosphere each year. The processes responsible for the large variations in emission rates found within and across wetland types have been studied. A strong relationship between $\mathrm{CH}_{4}$ emission and net ecosystem production has been found. This relationship has been observed not only within canopies of sawgrass (Cladium jamaicense), sedge (Carex sp.), and agricultural rice (Oryza sativa) but also across wetlands extending from subarctic peatlands to subtropical marshes. In each of these environments, approximately $3 \%$ of the net ecosystem production is emitted to the atmosphere as $\mathrm{CH}_{4}$. This research suggests that, in addition to water level, primary production may be a master variable integrating many factors controlling $\mathrm{CH}_{4}$ emission across vegetated wetlands. Factors determining these relationships will be studied.

\section{HEAT FLUXES IN A COUPLED ATMOSPHERE-ATLANTIC OCEAN MODEL IN RESPONSE TO VARIATIONS IN IMPOSED TEMPERATURE GRADIENTS}

\section{CHASSIGNET, ERIC}

\section{UNIVERSITY OF MIAMI}

An atmospheric global spectral model (AGSM) has been coupled to an isopycnic-coordinate model of the North Atlantic Ocean to investigate the relative roles played by the atmosphere and the Allantic Ocean in transporting heat poleward under different radiative equilibrium, or greenhouse, scenarios. As different radiative-equilibrium temperature gradients are imposed on the atmosphere component, how the poleward heat fluxes in both the 
atmospheric and oceanic components respond to regulate the coupled-model climate is studied. Results have been obtained from a suite of equilibrium integrations (where the forcing is fixed for the duration of integration) and from transient experiments (where the forcing is continuously increasing or decreasing over the integration). The nature of climate drift in the coupled model has been investigated with results from a parallel suite of uncoupled model integrations. The transient experiments have allowed investigation of how the heat fluxes are partitioned over time in response to the radiative perturbation. These experiments also serve to test an algorithm that attempts to recover the equilibrium solutions from the output of a transient experiment.

\section{CLIMATE-MODEL VALIDATION WITH MSU GLOBAL TEMPERATURES}

\section{CHRISTY, JOHN R.}

\section{UNIVERSITY OF ALABAMA, HUNTSVIILE}

Since late 1978, the microwave sounding units (MSUs) have monitored deep-layer temperatures for the entire globe to very high precision. Using the MSU data, we have begun to compare GCM output with the MSU temperatures over the common AMIP period of 1979 to 1988. The first model data made available from LLNL were produced by the European Center for MediumRange Forecasts (ECMRF) model as modified by the climate-research group in Hamburg, Germany. Because each model is executed on its own spatial grid, considerable programming is required to accurately interpolate the regular MSU gridded data to the grid of the model. Once this was done for the ECMRF data, comparison tests were developed, and results of the comparisons were obtained. The AMIP office has informed us that a second model run will be made available to us, this one from the Canadian Climate Center. We will continue to compare output from the various models over the 1979-1988 study period and to assess signal magnitude with the goal of reducing model uncertainty.

PRODUCTION AND SEA-AIR FLUX OF CARBONYL SULFIDE IN THE COASTAL ENVIRONMENT

CUTTER, GREGORY

OLD DOMINION UNIVERSITY
Carbonyl sulfide (OCS) is the primary source of sulfate aerosols in the stratosphere and therefore affects the global radiation balance. The two largest inputs of OCS to the atmosphere are fossil-fuel combustion and oceanic emissions, with most of the oceanic input probably coming from the coastal zone. The temporal variability of OCS sea-air fluxes in estuaries has been examined, and sediments have been found to be a source of OCS, so sediment-water fluxes are also being measured. Measurements of OCS fluxes will be made in a variety of coastal regimes, and laboratory experiments will be conducted to reveal the rates and mechanisms producing OCS in coastal sediments.

\section{DYNAMIC ALLOCATION OF RESOURCES FOR THE MITIGATION OF THE EMISSION OF GREENHOUSE GASES FROM THE TRANSPORTATION AND ELECTRIC UTILITY SECTORS}

\author{
GREENING, LORNA
}

\author{
UNIVERSITY OF ALABAMA
}

On the average, approximately $6 \%$ of U.S. consumers' total annual expenditures go to transportation and residential electricity usage. Any attempt to mitigate the emission of greenhouse gases (GHG) will impact consumers directly and indirectly, either through increased costs or through altered life-styles. The implementation of possible policy and technological alternatives across these two energy end-use sectors will be considered. A dynamic programming formulation will be used to develop a coordinated, cross-sector approach to the reduction of greenhouse gases emitted as a result of consumer demand for household electricity and transportation. A planning horizon of 50 to 75 years will be used.

To incorporate regional availability of supply options, full-fuel-cycle analyses that have been developed previously on a regional basis will be used. Demand for both electricity and transportation services on a consumer-unit basis will be developed for the Southeastern region. As a result of this approach, policies for various targeted levels of reduction of greenhouse gases from these two end-use sectors can be determined. A cross-sector approach should result in more efficient implementation than individual-sector implementation. 


\section{HYDROCARBON EMISSIONS FROM SOUTHERN PINES AND THE POTENTIAL EFFECTS OF GLOBAL CLIMATE CHANGE}

\author{
JOHNSON, JON \\ UNIVERSITY OF FLORIDA
}

\section{MATHEMATICAL MODELING OF THE COUPLED KATABATIC, OCEAN, AND ICE SYSTEMS OF THE ANTARCTIC}

\author{
MCNIDER, RICHARD \\ UNIVERSITY OF ALABAMA, \\ HUNTSVILLE
}

Nationwide, trees have been estimated to contribute between 30 and 60 billion $\mathrm{kg}$ of carbon per year to the atmosphere, affecting air quality and regional climate. To riucidate these effects for the southeast United States, accurate emission rates, as affected by temperature and organ (foliage and stem), will be determined over a range of different-aged loblolly and slash pine trees, and how the quantity and quality of hydrocarbon emissions may be affected by elevated temperature, ozone, drought, and $\mathrm{CO}_{2}$ will beinvestigated.

Long-term studies will be conducted under both controlled-environment and chamber-modified field conditions. Parameters being measured will provide a clear understanding of the quality and quantity of hydrocarbon emissions as affected by species, ontogeny, phenology, and organ area. Determining the interplay between emissions and organ area will be important for future attempts at scaling up to stand, ecosystem, and regional levels.

\section{SATELLITE STUDY OF URBAN WARMING FOR GLOBAL-CLIMATE-CHANGE RESEARCH}

\author{
KIDDER, STANLEY \\ UNIVERSITY OF ALABAMA, \\ HUNTSVILLE
}

Surface-based thermometer data have problems that must be corrected for before such time series can be properly evaluated. One such problem is that of urban warming. As a city grows to surround the site of its weather station, a false warming signal may become part of the temperature record. Before the record can be evaluated, this urban-warming signal must be removed. Infrared data from weather satellites (specifically, data from the AVHRR on NOAA satellites) will be used with surface-temperature observations to quantify the magnitude of the urban-warming effect and to develop a correction for it.
Atmospheric GCMs perform particularly poorly for the Antarctic region. There, katabatic winds driven by the sloped topography of the Antarctic continent dominate the atmospheric circulation over the continent and adjacent coastal areas and may strongly influence coastal oceanography and ice movement. A mesoscale coupled ocean-atmospheric model will be developed and applied to the katabatic and coastal ocean systems of the Antarctic. Preliminary results show that the katabatic winds adjust rapidly to the geostrophic direction (easterly), especially over the ocean. This directionality produces a substantial latitudinal curl in the easterly wind stress, which in turn produces strong downwelling near the coast and strong upwelling $300 \mathrm{~km}$ offishore in the ocean model. Based on these results, the contribution of the katabatic winds to the latitudinal curl is a significant component in maintaining the Antarctic divergence zone, which is responsible for the high biological productivity in the Southern Ocean. This complex ecosystem may be highly vulnerable to changes in the katabatic wind regime caused by increased Antarctic cloudiness under certain climate-change scenarios.

\section{WATER-USE OF MANAGED SOUTHERN PINE AND NATURAL HARDWOOD FORESTS}

\section{OREN, RAM}

\section{DUKE UNIVERSITY}

Because global warming is expected to increase the water use of trees, the annual water use by pine and hardwood stands of the Piedmont region will be quantified. Pinus taeda (L.) stands of two age classes have been equipped with a micromet-xylem flux system. Early data showed daily stand transpiration ranging from 1 to $2.7 \mathrm{~mm}$ and strongly correlated with the canopy's vapor pressure deficit. These transpiration values are similar to those reported for Bavarian Picea abies L. Karst. stands with three to four times the leaf-area index. Thus, $P$. taeda uses a relatively large quantity of water per unit of leaf area. Moreover, the effect of precipitation on transpirational water use in these stands 
was large. The early data indicated that pine forests are very sensitive to even short-duration drought during the growing season, indicating stomatal closure and, consequently, lower $\mathrm{CO}_{2}$ uptake. A free-air $\mathrm{CO}_{2}$ enrichment ring will be used in a $P$. taeda stand and in a natural hardwood stand to study whether an increase in atmospheric $\mathrm{CO}_{2}$ concentration could compensate for a temperature increase unaccompanied by an increase in precipitation and whether broadleaf species are as sensitive to short-duration droughts as are pines.

\section{RESPONSE OF LONGLEAF PINE TO ELEVATED ATMOSPHERIC CO $\mathrm{CO}_{2}$ : RESOURCE AVAILABILITY, CARBON PARTITIONING, AND RESPIRATION}

$$
\text { ROGERS, HUGO }
$$

\section{AUBURN UNIVERSITY}

Multiple resource limitations may constrain plant response to $\mathrm{CO}_{2}$ in terms of carbon production, respiration, and allocation, and $\mathrm{CO}_{2}$ response may be mediated through community interactions, including competition, insect herbivory, and plant diseases. Much of the state of knowledge on community responses is based on short-term studies with herbaceous plants, but these changes are particularly important for forest communities because of the long-lived nature of trees and their economic importance. To determine how plant response at elevated $\mathrm{CO}_{2}$ levels is modified by other environmental factors, such as moisture and nutrient stresses, longleaf pine seedlings will be exposed to two levels of $\mathrm{CO}_{2}$ in open-topped chambers. Two nitrogen levels and two water-potential levels will be employed. Seedlings will be harvested four times to allow assessment of phenological aspects of root development. At each harvest, plant component parts will be assessed for photosynthate partitioning, total carbon and nitrogen will be analyzed, and carbon partitioning and nitrogen uptake will be calculated. Carbon flux will be determined in the plant component parts and in the root-soil system, and carbon construction and maintenance costs will be determined. Whole-plant water use will be assessed gravimetrically.

\section{CLOUD-CLIMATE-FEEDBACK MECHANISMS: IMPACT OF REDUCTION IN FOSSIL-FUEL EMISSIONS}

\author{
SAXENA, VINOD
}

\section{NORTH CAROLINA STATE UNIVERSITY}

To assess the effects of anthropogenic sulfate aerosols on levels of $\mathrm{CCN}$ and thus on the cloud-mediated albedo (as measured with the AVHRR), available data sets were analyzed. An analysis of the CCN concentration, cloud-droplet size distribution, and liquidwater content of clouds observed at Mount Mitchell, N.C. (the highest peak east of the Mississippi River) showed that large variations in $\mathrm{CCN}$ concentration are associated with the boundary of different air masses while smaller changes occur with the air-mass transport. Data on California stratocumulus clouds and Arctic stratus clouds showed that local CCN can be enhanced by a factor of four within photochemically active clouds largely counteracting the greenhouse-warming effect from a doubling of $\mathrm{CO}_{2}$. A kinetic cloud-chemistry model was developed to examine the dependence of the cloud-water acidity on droplet size. It showed that smaller droplets have higher $\mathrm{pH}$ values even though sulfate-ion concentrations in them are much higher than in larger droplets, presumably resulting from the presence of carbonate aerosols. The model simulations showed a good qualitative agreement with the observations at Mt. Mitchell. To understand the impact of reduction in sulfur emissions on cloud albedo, a diagnostic model was used; it found that a $2 \%$ per year reduction in the anthropogenic emission of $\mathrm{SO}_{2}$ would reduce the radiative forcing by clouds, resulting in warming, while an identical growth in $\mathrm{SO}_{2}$ emission would have a larger positive contribution, resulting in cooling. The $3.7-\mu \mathrm{m}$ reflectance of clouds provided by AVHRR data and the relationship of that reflectance with the physicochemical properties of clouds will be examined.

\section{EFFECTS OF RAINFALL MODIFICATION ON PLANT-HERBIVORE PROCESSES}

\author{
SHURE, DONALD J.
}

\section{EMORY UNIVERSITY}

Possible changes in plant allocation to foliar nutrients and phenolic defenses following rainfall manipulations will be examined, and the type and extent of insect 
herbivory from trees experiencing wet, ambient, or drought conditions will be quantified. Seedling, sapling, and mature stages of white oak (Quercus alba), chestnut oak (Q. prinus) and red maple (Acer rubrum) trees will be sampled to determine which stage of the life cycle of each species is most susceptible to herbivore attack while experiencing different moisture regimes. Results from the pretreatment phase of the experiment have indicated consistent differences in leaf nitrogen and phenolic defenses among the tree species being studied but no clear changes from seedling to mature life-cycle stages of each species. Insect herbivory ranged from $6.0 \%$ in red maple to $12.4 \%$ in white oak with strip feeders and leaf skeletonizers the major insect feeding guilds. The extent and type of herbivory was quite similar from seedling, sapling, and mature stages of each species. Pretreatment levels of leaf nitrogen, plant phenolics, and insect herbivory were also very similar when comparing wet-, ambient-, and dry-designated plots, thereby validating the original site-selection process.

\section{MODELING PATTERNS OF $\mathrm{CO}_{2}$ FLUX FROM FOREST ECOSYSTEMS}

\author{
SMITH, THOMAS M.
}

\section{UNIVERSITY OF VIRGINIA}

A method for exploring the short- and long-term consequences of a climatic change on the net flux of $\mathrm{CO}_{2}$ between the atmosphere and terrestrial surface will be developed. A combination of an individual-based forest-gap model and an ecosystem- $\mathrm{CO}_{2}$-flux model will be used to examine the long-term dynamics of vegetation structure and composition and the associated changes in the seasonal trends of net $\mathrm{CO}_{2}$ flux. The approach will be applied to a range of forested ecosystems in eastern North America representing a latitudinal gradient from boreal to tropical forest. The approach will allow testing the results from climate models that predict shifts in ecosystem distribution and variations in the uptake and release of carbon.

\section{SOUTHCENTRAL REGIONAL CENTER}

\section{PREDICTION OF GLOBAL METHANE PRODUCTION BY WETLANDS}

\author{
BHATTACHARYA, SANJOY; LAW, VICTOR; \\ DELAUNE, RONALD D.; and REDDY, $K . R$.
}

\section{TULANE UNIVERSITY, LOUISIANA STATE UNIVERSITY, and UNIVERSITY OF FLORIDA}

A systematic strategy will be developed for predicting global $\mathrm{CH}_{4}$ emissions from natural wetlands. Several parameters have been identified that are believed to affect generation and transport to the atmosphere. Laboratory and field studies will be conducted to determine the most important parameters that influence the global $\mathrm{CH}_{4}$ budget. Soils and organic matter present in wetlands will be characterized, and environmental conditions will be evaluated with respect to $\mathrm{CH}_{4}$ emissions. Laboratory studies will be carried out to determine kinetic parameters for $\mathrm{CH}_{4}$ production from organic matter. Relevant data, including soil samples, from different parts of the globe will be collected and characterized into a database on global $\mathrm{CH}_{4}$ emissions. $\mathrm{A}$ process-level model will be developed and integrated with the database to assist in predictions of global $\mathrm{CH}_{4}$ emissions from wetlands.

\section{INCORPORATING TRACERS IN THE HYDROLOGIC CYCLE OF THE GENESIS EARTH-SYSTEM MODEL}

COLE, JULIA, and WEBB, ROBERT

\section{UNIVERSITY OF COLORADO}

Isotopic and geographic tracers will be incorporated into the hydrologic cycle of the GENESIS Earth-system model (ESM) at NCAR, and initial control runs, sensitivity tests, and paleoclimatic model-validation experiments will be performed. The new tracer capability will (1) enable an entirely new class of observations (isotopic data) to be used for model validation of hydrologic parameterizations in the atmosphere, boundary layer, and land surface; (2) produce processoriented insight into observed isotopic changes in paleoclimatic records; and (3) provide a much-needed opportunity to compare results from different tracer-capable GCMs to evaluate the realism of tracer-model simulations, especially of such factors as vapor-source contributions that cannot be measured. This effort complements a parallel project at NASA's Goddard Institute for Space Studies to include tracers in upgraded atmospheric, surface-boundary-layer, and ground-hydrology components of their GCM. 


\section{THERMODYNAMIC FEEDBACK PROCESSES BETWEEN THE ATMOSPHERE AND SEA ICE}

\section{CURRY, JUDITH, and WEBSTER, PETER \\ UNIVERSITY OF COLORADO}

Adequate treatments of the thermodynamics of sea ice and the modeled thermodynamic feedbacks occurring among the atmosphere, ocean, and sea ice will be developed by constructing a detailed 1-D model of the coupled ocean-sea-ice-atmosphere system. An important focus of this research will be the thermodynamics feedback occurring between the cloudy atmospheric boundary layer and the sea ice. The issue of cloud, aerosol, and radiation parameterization in the Arctic will be addressed. Sensitivity tests will be conducted to determine the level of detail required by global climate models to address adequately the response of the Arctic sea ice and atmosphere to increasing atmospheric concentrations of greenhouse gases and aerosols. Where possible, simplified parameterizations will be developed for 3-D climate models.

\section{CLIMATE CHANGE IN NORTH CENTRAL OKLAHOMA FROM ANALYSIS OF BOREHOLE TEMPERATURES}

DEMING, DAVID

\section{UNIVERSITY OF OKLAHOMA}

Temperatures in the. solid Earth are a direct thermophysical consequence of climatic conditions prevailing at the air-ground interface, and valuable information on climate change over the past few hundred years can be obtained from the analysis of borehole temperature logs. Temperature logging will be carried out for six wells, each $\sim 425 \mathrm{~m}$ deep, and the resulting temperature data will be analyzed to constrain secular climatic trends in North Central Oklahoma over the past few hundred years. The wells were originally drilled by British Petroleum for the purpose of studying reservoir characterization, were recently turned over to the University of Oklahoma, and now offer an unusual and fortuitous opportunity to collect important information on climatic change at a relatively small cost. Collecting and analyzing temperature data from these boreholes will be straightforward but must be completed before the wells are flooded by a planned saltwater injection that will perturb the ambient temperature regime and destroy the usefulness of the wells for climatic studies.

\section{CLIMATE SENSITIVITY, PALEOCLIMATE, AND CLIMATE-MODEL VERIFICATION}

\author{
HOFFERT, MARTIN, and RAMPINO, MICHAEL
}

\section{NEW YORK UNIVERSITY}

Paleoclimate data will be used to verify and calibrate climate models with the goal of narrowing the uncertainty range of greenhouse-gas-induced global warming derived from those models. Three objectives will be pursued: (1) to put existing paleoclimate studies (for example, CLIMAP, COHMAP, and particularly recently available data derived by USSR paleoclimatologists) into formats suitable for climatemodel verification and calibration; (2) to compare paleoclimate data with results of schematic energybalance models (EBMs) with an emphasis on how global climate sensitivity and equator-to-pole temperature gradients depend on poleward-heat-flow feedback; and (3) to explore how subgrid parameterizations of GCMs can be modified to recover the paleoclimate sensitivity and equator-to-pole temperature changes (including boundary-layer, atmospheric, and ocean subgrid eddy-diffusivity, cloudformation, and cloud-radiation parameterizations). The radiative forcing (solar, albedo, and greenhouse gas) will be reconstructed as well as the pattern of temperature response of the paleoclimates in question. Efforts will focus on analysis and verification of new Russian paleotemperature data, models and data for $\mathrm{CO}_{2}$ variations over the Phanerozoic Eon, and climate-model sensitivity to poleward-heat-flow feedback.

\section{PREDICTION OF GLOBAL METHANE EMISSIONS FROM RICE AGRICULTURE}

\section{LAW, VICTOR, and BHATTACHARYA, SANJOY}

\section{TULANE UNIVERSITY}

Process-level modeling aimed primarily at the kinetics of $\mathrm{CH}_{4}$ production in rice soils and the subsequent fate of the $\mathrm{CH}_{4}$ will be used to provide estimates of global $\mathrm{CH}_{4}$ emissions from rice paddies. Current models replicate the seasonal peaks usually observed in $\mathrm{CH}_{4}$ prodıction and with only modest tuning show excellent agreement with experimental data. To make these models more robust, (1) their simple mass-transfer- 
coefficient aspects will be replaced with more precise components that use multicomponent diffusion and the activity of methane-consuming bacteria; (2) they will be combined with existing rice-plant models so root sloughing and aereenchyma geometry are considered directly; and (3) a global database of existing data on flooded rice production will be constructed and integrated with the models. The final package will be a userfriendly, personal-computer-based system that can be used to simulate various scenarios aimed at reducing $\mathrm{CH}_{4}$ emissions from rice agriculture while maintaining or increasing yields.

\section{METHANE EMISSIONS AND MITIGATION FROM LOUISLANA AND PHILIPPINES RICE AGRICULTURE}

LINDAU, C. W.; DELAUNE, R. D.; and PATRICK, W. H., JR.

\section{LOUISIANA STATE UNIVERSITY}

Methane production and emissions will be quantified, and proposed $\mathrm{CH}_{4}$-mitigation actions (i.e., selected compounds and alternations of rice-production practices) on rice ecosystems in Louisiana and the Philippines will be assessed with both field and greenhouse experiments. Field research will be conducted at the Rice Research Station, Crowley, La., and at the International Rice Research Institute (IRRI), Laguna, Philippines. Data collected from Louisiana ond the Philippines will be made available to experimental modelers constructing a global $\mathrm{CH}_{4}$ data base and prediction model.

\section{SOIL-ATMOSPHERE EXCHANGE OF METHANE FROM NATURAL AND AGRICULTURALLY IMPACTED RIPARIAN WETLANDS}

\section{MEYER, JUDITH, and BURKE, ROGER}

\section{UNIVERSITY OF GEORGIA}

The observed atmospheric $\mathrm{CH}_{4}$ increase may be due to both increases in $\mathrm{CH}_{4}$ sources and to decreases in $\mathrm{CH}_{4}$ sinks. Fertilization may increase $\mathrm{CH}_{4}$ emission from natural wetlands, and soil disturbance (either physical or chemical) has been shown to decrease the uptake capacity of some soils. Faciors that influence the magnitude and direction of the flux of $\mathrm{CH}_{4}$ between soils and the atmosphere (including soil temperature and moisture level, soil disturbance resulting from conventional-till and no-till agricultural practices, and soil-nutrient enrichment resulting from fertilizer and waste application and runoff) will be studied. In addition, stable-carbon-isotope compositions of $\mathrm{CH}_{4}$ will be determined on selected samples as an indicator of processes that influence soil-atmosphere $\mathrm{CH}_{4}$ exchange. The stable-isotope data will also be used in isotopemass-balance models of the atmospheric-methane cycle.

\section{RAPID CLIMATE CHANGE}

\author{
MORANTINE, MICHAEL, and WATTS, ROBERT
}

\section{TULANE UNIVERSITY}

Interactions among the cryosphere, the oceans, and the atmosphere will be studied to understand and predict climate change. The most recent period in the history of the Earth (between 14,000 and 9,000 years ago) when very dramatic and well-documented climate shifts ocsurred will be focused on. Insights about how these subsystems of the climate interact with each other during transition will be attained.

\section{SEASONAL CYCLE OF VARIABILITY OF SURFACE TEMPERATURE: STUDIES WITH EBMs AND DATA \\ NORTH, GERALD}

\section{TEXAS A\&M UNIVERSITY}

The variance and covariance properties of the fluctuations of the surface temperature field exhibit a seasonal cycle. Empirical studies have shown that the amplitude of these fluctuations are larger in the winter hemisphere. This result is reasonable because it is wellknown that the winter hemisphere has more potential for atmospheric flow instabilities. The fluctuations are larger over the land masses. This latter fact is consistent with the lower effective thermal inertial over these areas. These phenomena will be examined in detail first with data and then by fitting with simple phenomenological stochastic climate models. Identification and construction of such models are useful in signal and noise studies in the detection of forced climate change and in the error assessments for various observational-system designs. 


\section{FACTORS AFFECTING FORMATION, EMISSION, AND SECONDARY REACTIONS OF NITROUS OXIDE AND METHANE IN THE FLOODED SOIL PROFILES OF GULF COAST WETLANDS AND FLOODED RICF FIELDS}

\author{
PATRICK, WILLIAM H., JR.

\section{LOUISIANA STATE UNIVERSITY}

Because of the difference in soil-redox conditions necessary for the reduction of nitrate to nitrous oxide and the reduction of $\mathrm{CO}_{2}$ to $\mathrm{CH}_{4}$, nitrous oxide should be formed near the surface of flooded soils and lake sediments, and $\mathrm{CH}_{4}$ should be formed deeper. Consequently, nitrous oxide should be more subject to movement to the atmosphere by means of ebullience and diffusion than the $\mathrm{CH}_{4}$. If the soil is drained, this $\mathrm{CH}_{4}$ can be either microbiologically oxidized by oxygen diffusing downward through the drained soil or lost to the atmosphere by diffusing upward through the drained soil. How much of the nitrous oxide and $\mathrm{CH}_{4}$ formed in the flooded soil profile remains trapped in the soil and how much is emitted to the atmosphere will be determined. How much of the entrapped $\mathrm{CH}_{4}$ is lost to the atmosphere and how much is oxidized when a flooded soil is drained will also be determined.

\section{TRACE-GAS EXCHANGE WITH THE ATMOSPHERE FROM GULF COAST AGRICULTURAL WETLANDS}

SASS, RONALD L., and FISHER, FRANK M.

\section{RICE UNIVERSITY}

The annual rate of and seasonal changes in the production, oxidation, and emission of atmospheric trace gases from rice fields have been measured. The dependence of $\mathrm{CH}_{4}$ production and emission on rice ecosystem factors has been characterized. The source, size, spatial and temporal variation in, and rate of use of various carbon substrate pools used by methanogenic and methanotropic bacteria have been determined. These and similar process studies, including the extensive study of $\mathrm{CH}_{4}$ oxidation processes, will be continued and incorporated into a general, dynamic, box model of methanogenic processes and exchange with the atmosphere to obtain predictions of methane-emission characteristics under different ecological conditions.

\section{DETECTION OF GREENHOUSE CLIMATIC CHANGE}

\author{
WATTS, ROBERT G.
}

TULANE UNIVERSITY

Climatic variability will be studied by examining the outputs of unforced runs of climatic models of various complexity; cloud and radiation data from ERBE, Nimbus-6, Nimbus-7, and Meteor; and the COADS data set for ocean heat flux. Examining the model-output data will be useful in understanding the roles of the various parameterizations of cloud variability, oceanatmosphere coupling, etc. in the sensitivities and variabilities of the models. Examination of the satellite data sets will give information on the actual variability of the radiative heating at the top of the atmosphere and on interannual cloudiness variability. If it is possible to obtain useful data for ocean-heat-flux variability from the COADS data set, it will be invaluable in indicating to what extent the variability of the state of the ocean is responsible for interdecadal variability of the global surface temperature.

\section{IMPORTANCE OF CLIMATE FEEDBACKS IN DETERMINING FUTURE METHANE CONCENTRATIONS}

\author{
WIGLEY, T. M. L.
}

\section{UNIVERSITY OF COLORADO}

Terrestrial $\mathrm{CH}_{4}$ emissions are strongly influenced by temperature and soil-moisture levels. Greenhouse-gasinduced climate change will therefore affect these emissions and feed back into the climate system. This highly uncertain feedback will be quantified by developing and using a coupled methane-cycle-climate model to assess its likely importance relative to other factors and to estimate its uncertainty range. The 1-D upwelling-diffusion energy-balance model of Wigley and Raper (which separatis the globe into land and ocean boxes in each hemisphere) will be used. A parameterized methane-cycle model, in which methane-lifetime variations are determined by the concentrations of $\mathrm{CO}$, $\mathrm{NO}_{x}, \mathrm{NMHCs}$, and $\mathrm{CH}_{4}$, will be synchronously coupled to the climate model. Emission feedbacks will be included by making an appropriate fraction of total $\mathrm{CH}_{4}$ emissions a function of temperature change over the relevant box of the model, scaled to account for regional 
and seasonal dependence with GCM results. The contribution of the feedback to future, transient, global, mean temperature and sea-level change will be com- pared with other contributions for a wide range of climate and $\mathrm{CH}_{4}$ model parameters and scenarios of future anthrogenic $\mathrm{CH}_{4}$ emissions.

\section{Western Regional Center}

\section{SINK-SOURCE CHARACTERISTICS OF TWO DISTINCTLY DIFFERENT FOREST SPECIES AS AFFECTED BY ELEVATED CARBON DIOXIDE (PHASE II: INTERACTIONS WITH AVAILABLE SOIL MOISTURE)}

PUSHNIK; JAMES C., and DEMAREE, RICHARD

\section{CALIFORNIA STATE UNIVERSITY AT CHICO}

An integrated multidisciplinary study will be conducted to evaluate the responses of two forest species: Pinus ponderosa, an economically important species, and Taxus brevifolia, a species prized for production of anticancer compounds. This study will examine the interactive effects of elevated $\mathrm{CO}_{2}$ and soil moisture on the plant's carbon source/sink relationships. Specifically, it will examine carbon assimilation and transpiration (gas exchange- $A / C_{i}$ curves and temperature effects, RuBPCase activity and content, and chloroplast structure and integrity); transport functions (SPS activity and content and phloem and xylem structure and development); carbo sinks (taxol, growth, and biomass); and their interactions. The plants will be exposed to three $\mathrm{CO}_{2}$ levels (ambient, ambient $+175 \mu \mathrm{L} / \mathrm{L}$, and ambient + $350 \mu \mathrm{L} / \mathrm{L})$ and up to three levels of soil moisture $(-0.3$ to $-0.6 \mathrm{MPa},-1.0$ to $-1.3 \mathrm{MPa}$, and -2.0 to $-2.5 \mathrm{MPa}$ ).

\section{RELATIONSHIPS AMONG CCN CHEMISTRY, CCN SOURCES, CLOUD MICROPHYSICS, AND PRECIPITATION IN THE EASTERN SIERRA NEVADA}

BORYS, RANDOLPH D., and LOWENTHAL, DOUGLAS H.

\section{DESERT RESEARCH INSTITUTE}

The effect of different sources of $\mathrm{CCN}$ on snowfall rates in the Sierra Nevada will be studied to see if varying the numbers of CCN and alteration of the cloud-droplet size distribution will affect snowfall rates. Cloud water will be collected on Mt. Rose in the Carson Range in western Nevada and will be analyzed for trace elements and anion chemistry. Cloud-droplet concentrations and size distributions will be measured continuously with a PMS-FSSP during cloud-droplet-collection periods. Chemical analyses of the cloud water will be used to measure the chemical composition of the precloud CCN. Source apportionment of the chemical constituents of the cloud droplets to natural and anthropogenic sources (including power plants, motor vehicle exhaust, geological dust, and marine aerosol) will be done with receptor modeling. The measured cloud microphysical data will be used in a snow-growth model to predict snowfall rates. The measured and predicted snowfall rates will be compared and used to calibrate the model. The combined observational and model results will be used to assess the potential effect of changing concentrations of $\mathrm{CCN}$ on winter snowpack in the Sierras.

\section{ENVIRONMENTAL INFORMATION FROM THE ISOTOPIC COMPOSITION OF HYDROGEN AND CARBON IN THE WESTERN UNITED STATES AND ALASKA}

EPSTEIN, SAMUEL

\section{CALIFORNIA INSTITUTE OF TECHNOLOGY}

The $\mathrm{D} / \mathrm{H}$ ratios of cellulose extracted from plants is largely determined by the $\mathrm{D} / \mathrm{H}$ ratios of the meteoric water incorporated by the plants, which in turn are systematically related to the temperature at the site of precipitation. Thus, the $\mathrm{D} / \mathrm{H}$ ratios in trees can be used as a proxy indicator of the past climatic temperature. The D/H ratios of trees in the west coast areas and Alaska will be analyzed (1) to establish the relationship between the $\delta \mathrm{D}$ values of trees and the mean annual temperatures with good resolution and to understand how the $\delta D$ values of trees are spatially controlled by the air-mass movement and the $8 \mathrm{D}$ values of groundwater; (2) to reconstruct the climatic temperature records for at least the past 200 years in Northern California, Oregon, Alaska, and Washington; and (3) to 
detect natural climatic cycles and trends and thus identify the anthropogenic contributions to the climate change.

The 400 -year $\delta^{13} \mathrm{C}$ records of the bristlecone pine from the White Mountains has been found to match almost perfectly the $\delta^{13} \mathrm{C}$ records of a juniper tree from the Sinai Peninsula in Egypt, suggesting that both may be recording the $\delta^{13} \mathrm{C}$ variation in the atmospheric $\mathrm{CO}_{2}$. The $\delta^{13} \mathrm{C}$ records of trees grown in environments similar to that of these two trees may permit the reconstruction of the isotopic history of the atmospheric $\mathrm{CO}_{2}$.

\section{GLOBAL-WARMING TRENDS AND INTERNAL CLIMATE OSCILLATIONS: DETECTION AND MODELING}

\section{GHIL, MICHAEL, and NEELIN, J. DAVID \\ UNIVERSITY OF CALIFORNIA AT LOS ANGELES}

The existing records of surface air and sea temperature and surface winds will be decomposed into a trace-gasinduced trend, oscillatory components caused by natural interdecadal variability, and noise by applying an existing, advanced, statistical toolkit to univariate and multivariate time series of $\mathrm{CO}_{2}$ concentration and surface-climate variables. In addition, the dominant spatiotemporal patterns of interdecadal variability will be related to physical and biogeochemical mechanisms by developing further and applying to interdecadal variability a set of models from the ocean's thermohaline circulation coupled to sea-ice and to highly simplified atmospheric models. This will help isolate the natural variability of the atmosphere-ocean climate system from the human-induced climatic changes, leading to a better simulation and prediction of the latter.

\section{EARTHSHINE MEASUREMENTS OF THE GLOBAL ALBEDO}

KOONIN, S. E., and ZIRIN, H.

\section{CALIFORNIA INSTITUTE OF TECHNOLOGY}

The Earth's albedo will be studied through observations of earthshine. A coronagraph and a charged coupled device (CCD) system will be constructed and installed at the Big Bear Solar Observatory and used in nightly lunar observations in four wavelength bands spanning the solar shortwave spectrum. Analysis of these images and their correlation with meteorological data will establish the synoptic, seasonal, and interannual variability of the Earth's albedo to a precision of $0.2 \%$. Related modeling will also be conducted with GCMand satellite-derived cloud covers.

\section{PARSIMONIOUS PARAMETERIZATION OF LAND HYDROLOGY FOR CLIMATE MODELS}

LETTENMAIER, DENNIS P.

\section{UNIVERSITY OF WASHINGTON}

Detailed surface-moisture and energy-flux data available at two locations in the midcontinental United States will be used to determine the level of complexity needed in representations of land-surface hydrology to reflect properly the effects of subgrid variations in soil properties and topography on surface fluxes at the GCM scale. They will also be used to develop and validate an extended version of VIC, a parsimonious water-energybiospheric model suitable for inclusion in GCMs that parameterizes subgrid hydrologic heterogeneities that are of importance for water-, energy-, and soil-gas-flux prediction. VIC represents the effect of these heterogeneities on soil moisture through a statistical representation of the variation of soil-moisture capacities over an area. A second, more detailed local model will be used that explicitly reflects the effects of soil and topographic heterogeneities on runoff-production and surface-energy fluxes. The local model will be used to evaluate the extent to which the simplifications in VIC are justifiable on a large scale. Measured surface-energy and moisture fluxes from FIFE and the Southern Great Plains ARM site, extended with satellite observations, will also be used to evaluate the performance of the new version of VIC.

\section{REGIONAL CLIMATOLOGIES OF CALIFORNIA AND HAWAII} ROADS, JOHN O.; CHEN, SHYH-CHIN; and
STEVENS, DUANE

\section{SCRIPPS INSTITUTION OF OCEANOGRAPHY and UNIVERSITY OF HAWAII}

An attempt will be made to understand the climatology of California and Hawaii through the development 
and/or application of high-resolution and comprehensive climatological simulations to answer the question, how will the regional climatology of California and Hawaii differ under the impending global change? Efforts will focus on such issues as how much precipitation will fall in the U.S. West and California, whether this precipitation will fall mainly as rain or as snow, how the snow will be geographically distributed with respect to watersheds, how near-surface winds transport active and inert chemical substances through the mountains and valleys adjacent to urban areas in California, and the angle at which the trade winds impinge upon the mountains of Maui.

\section{ROLE OF THE OCEAN IN CLIMATE VARIABILITY: OCEAN-VENTILATION PROCESSES, THERMOHALINE VARIABILITY, AND FEEDBACKS OF THE OCEAN TO ICE AND TO THE ATMOSPHERE}

$$
\text { SARACHIK, E. S. }
$$

\section{UNIVERSITY OF WASHINGTON}

The role of the ocean in producing natural climate variability will be probed by investigating ocean-ventilation processes with a variety of ocean models, the basic mechanisms and sensitivities of thermohaline variability, atmosphere-ocean and ice-ocean feedback mechanisms on decadal and longer time scales, and their roles in inducing climate change, as evidenced in observed paleoclimatic records.' These investigations will be carried out with simulations of low-frequency variability with ocean GCMs in simplified contexts and with a geostrophic ocean GCM; experiments with a new, hybrid ocean model; analytic projection of ocean responses; model tracer studies; comparative studies with the Bryan-Cox ocean GCM; studies with a simplified atmospheric parameterization coupled to an ocean model; and studies of ice interacting with the ocean.

\section{CORRELATION OF MARINE AND CONTINENTAL PALEOCLIMATE RECORDS}

\author{
VEROSUB, KENNETH L., and \\ ROBERTS, ANDREW P.

\section{UNIVERSITY OF CALIFORNIA AT DAVIS}

Sequences of lake sediments in outcrops and cores from south-central Oregen have been studied. Distinct volcanic ash layers occur in each record tha: can be used for correlation and approximate age control. They have shown that it is possible to obtain a record of paleointensity of the geomagnetic field from lake sediments from the western United States and that it may be possible to correlate palcointensity variations on a global scale. The records from these sites are being compared with each other and will be compared with records soon to be obtained from marine cores from the northeast Pacific Ocean. If the paleointensity records match, the ${ }^{18} \mathrm{O}$ isotope stratigraphy of the marine core will be able to be transferred to the paleoclimate records from the lake sediments. This transfer will create a common time scale for marine and continental records and will permit us to compare climate processes in each environment.

\section{METHANE EMISSIONS FROM RUMINANT LIVESTOCK}

\section{WESTBERG, HAL; LAMB, BRIAN; and JOHNSON, KRIS}

\section{WASHINGTON STATE UNIVERSITY}

Methane emission rates from cattle will be measured under field conditions with a tracer technique. The methodology involves placing a sulfur hexafluoride permeation device in a cow's rumen and measuring $\mathrm{CH}_{4}$ and $\mathrm{SF}_{6}$ levels near the mouth. From a knowledge of the $\mathrm{SF}_{6}$ permeation rate and the recorded $\mathrm{CH}_{4}: \mathrm{SF}_{6}$ concentration ratio, the $\mathrm{CH}_{4}$ emission rate can be calculated. Methane releases from cattle groups that dominate U.S. production will be systematically determined. These data will provide the basis for improving current estimates of $\mathrm{CH}_{4}$ emissions to the atmosphere from ruminant livestock.

ANALYSIS OF THE MEASURED VARIABILITY IN INTEGRAL AEROSOL PROPERTIES IN THE MARINE BOUNDARY LAYER

$$
\text { CHARLSON, ROBERT J., and }
$$
COVERT, DAVID S.

\section{UNIVERSITY OF WASHINGTON}

The radiative effects and the underlying chemical and physical properties of tropospheric aerosols will be measured at a marine, midlatitude site on the northwest coast of the United States where the anthropogenic impact is minimized. These measurements will provide 
a characterization of the background aerosol to which anthropogenic aerosols are added. The work will have three goals: to acquire a complete, in fact redundant, set of chemical, physical and optical aerosol measurements over a long time period; to continue to refine the methods for providing provable comparable measurements over a network of sites and to aid in implementation at those sites; and to analyze the integrated aerosol parameters from other sites with those from this site. This collaboration is intended to determine the spatial variability of the aerosol and the effect of anthropogenic sources.

\section{PHYSICS AND PARAMETERIZATIONS OF CIRRUS CLOUDS}

\author{
FLATAU, PIOTR \\ SCRIPPS INSTITUTION OF \\ OCEANOGRAPHY
}

High-spectral-resolution radiometric data from the ARM Program, the First ISCCP Regional Experiment (FIRE), and the Central Equatorial Pacific Experiment (CEPEX) will be used to investigate the climate influence of cirrus clouds. The exact discrete-dipole-approximation (DDA) calculations will provide new single scattering parameters of the nonspherical particles for use in satellite cloud climatologies (ISCCP), retrievals from the high-resolution interferometry, and infrared satellite radiometric data. Detailed 1-D cirrus cloud models will be coupled with LBL radiative-transfer calculations to determine effective radius and predict spectral signatures of cloud microphysics. These results will improve cirrus parameterizations in climate models and cloud radiative feedback studies.

\section{DEVELOPMENT OF FIELD \\ INSTRUMENTATION FOR ATMOSPHERIC- OXYGEN MEASUREMENTS}

KEELING, RALPH F.

\section{SCRIPPS INSTITUTION OF OCEANOGRAPHY}

Current programs to measure trends in atmospheric oxygen are limited by the lack of field-deployable analyzers. $A$ field-deployable oxygen analyzer will be developed by modifying a commercially available Siemens paramagnetic analyzer that appears capable of attaining the required precision ( 1 or $2 \mathrm{ppmV}$ ). The analyzer will be extensively tested in simulations of background monitoring on air in Boulder. Its successful application will allow a better understanding of the rates and mechanisms that govern $\mathrm{CO}_{2}$ addition and removal from the atmosphere by the oceans and the land biosphere.

\section{COSMOGENIC ${ }^{10}$ Be AND ${ }^{36} \mathrm{Cl}$ AS TOOLS FOR DELINEATING EXTREME NATURAL PREHISTORIC VARIABILITY IN THE POLAR STRATOSPHERE}

\section{LAL, DEVENDRA}

\section{SCRIPPS INSTITUTION OF OCEANOGRAPHY}

The possibility will be explored of using the paleorecord of deposition of the cosmogenic radionuclides ${ }^{36} \mathrm{Cl}$ (half-life $=0.3$ million years) and ${ }^{10} \mathrm{Be}$ (half-life $=1.5$ million years) in polar ice to identify occurrences of extreme conditions in the polar stratosphere in the past (e.g., denitrification and enhanced ozone removal). In the preanthropocentric era these conditions may have produced by injections of volcanic $\mathrm{Cl}$ and $\mathrm{SO}_{4}$ aerosols.

Direct measurements of ${ }^{36} \mathrm{Cl}$ production rates by cosmic radiation will be made with two independent methods, and ${ }^{36} \mathrm{Cl}$ and ${ }^{10} \mathrm{Be}$ fallout in the polar regions will be modeled with realistic large-scale atmosphericcirculation and -mixing models.

\section{GLOBAL STUDIES OF TRACE-GAS CONCENTRATIONS IN URBAN LOCATIONS}

\author{
ROWLAND, F. SHERWOOD, and \\ BLAKE, DONALD R.

\section{UNIVERSITY OF CALIFORNIA AT IRVINE}

Air samples will be collected at ground level from many cities around the world, and excess $\mathrm{CH}_{4}$ in urban locations will be correlated to $\mathrm{CCl}_{2} \mathrm{~F}_{2}$ (CFC-12, whose emissions are known to be almost entirely urban) as a test of the $\mathrm{CH}_{4}$ source distribution. The excess $\mathrm{CH}_{4}$ has been found to be substantial in many of these cities and is believed to be the consequence of natural-gastransmission losses. The absolute scale of $\mathrm{CH}_{4}$ release is generaliy significantly smaller in the cities of the U.S. and western Europe than in the others sampled.

In these cities, the source strengths of methylchloroform $\left(\mathrm{CH}_{3} \mathrm{CCl}_{3}\right)$, carbon monoxide, and low-molecular-weight 
alkanes $\left(\mathrm{C}_{2} \mathrm{H}_{6}, \mathrm{C}_{3} \mathrm{H}_{8}\right.$, etc.) will also be studied. Measurements of urban concentrations of molecules (such as $\mathrm{CH}_{3} \mathrm{Cl}_{3}$ and $\mathrm{CCl}_{2}-\mathrm{CCl}_{2}$ ) will furnish useful comparisons with concentrations in remote regions and will serve as prototypes for the behavior in the atmosphere of the proposed hydrohalocarbon substitutes for the CFCs [e.g., $\mathrm{CHClF}_{2}$ (HCFC-22), $\mathrm{CH}_{2} \mathrm{FCF}_{3}$ (HFC-134A), and $\mathrm{CHCl}_{2} \mathrm{CF}_{3}$ (HCFC-123)].

\section{SPATIAL AND TEMPORAL DISTRIBUTIONS OF METHANE SOURCES}

TUNG, KA KIT

\section{UNIVERSITY OF WASHINGTON}

The temporal and spatial distribution of $\mathrm{CH}_{4}$ sources will be determined with an objective inversion algorithm and a 2-D chemical-transport model. Singular-value decomposition will be used to systematically identify those components of the global $\mathrm{CH}_{4}$ budget that are well defined by observations of atmospheric $\mathrm{CH}_{4}$ at the Earth's surface. The singular-value decomposition will then be used to identify independent information, taken from geographical and isotopic studies, capable of constraining those components of the global $\mathrm{CH}_{4}$ emis- sions that are poorly defined by the observations of atmospheric $\mathrm{CH}_{4}$.

\section{COMPARISONS OF OBSERVED AND MODEL CLOUDINESS AND MODEL CLIMATE SENSITIVITY}

WEARE, BRYAN C.

\section{UNIVERSITY OF CALIFORNIA AT DAVIS}

Observations of monthly mean cloudiness and its variability will systematically be compared with most of the world's current climate models. Those models will then be ranked according to ability to match the observed statistics. Thereafter, the climate sensitivity of the models will be calculated and compared to the goodness of fit between the model and observed cloud fields. The goal is to both identify which models best represent observed variations in cloudiness and indicate how agreement or lack of agreement with observations is likely to influence model sensitivity. The cloud observations will be drawn primarily from those of the International Satellite Cloud Climatology (ISCCP). Model-derived cloudiness will be drawn from the approximately 30 GCMs and forecast models taking part in the AMIP. 


\section{APPENDIX A: ADDRESSES OF PRINCIPAL INVESTIGATORS}

Ackerman, Thomas P.

Pennsylvania State University

516 Walker Bldg.

University Park, PA 16802

814-865-2915

Acock, Basil

Bldg. 007, Rm. 56, BARC-West

USDA-ARS Agricultural Systems Research Institute

Beltsville, MD 20705

601-323-2230 Ext. 62

Adams, John L.

RAND

1700 Main Street, P.O. Box 2138

Santa Monica, CA 90407-2138

310-393-0411 ext 7917

Allen, L. H.

USDA-ARS Plant Science Research Laboratory

Agronomy Department

University of Florida

Gainesville, FL 32611

904-392-6180

Anderson, John R.

Space Science and Engineering Center

University of Wisconsin

Madison, WI 53706

608-262-0783

Arakawa, Akio

Department of Atmospheric Sciences

University of California at Los Angeles

Los Angeles, CA 90024-1565

310-825-1852

Asher, William E.

Battelle Marine Sciences Laboratory

439 West Sequim Bay Road

Sequim, WA 98232

206-683-4151
Avissar, Roni

Department of Meteorology and Physical Oceanography

P.O. Box 231

New Brunswick, NJ 08903-0231

908-932-9387

Bader, David C.

ER-74

Office of Health and Environmental Research

USDOE

Washington, D.C. 20585

301-903-4328

Baer, Ferdinand

Department of Meteorology

University of Maryland

College Park, MD 20742

301-405-5387

Baerwald, Thomas J.

National Science Foundation

18th and G St NW

Washington, DC 20550

202-357-0357

Barnes, Fairley

Los Alamos National Laboratory, MS J-495

P.O. Box 1663

Los Alamos, NM 87545

505-667-3331

Barnett, Tim P.

Climate Research Division

Scripps Institution of Oceanography

La Jolla, CA 92093-0221

619-534-3223

Baumgardner, John R.

Los Alamos National Laboratory, MS B-216

P.O. Box 1663

Los Alamos, NM 87545

505-667-9102 
Bazzaz, F, A.

Department of Organismic and Evolutionary Biology Harvard University

Cambridge, MA 02138

617-495-2305

Bingler, Linda

Battelle Marine Sciences Lab

439 West Sequim Bay Road

Sequim, WA 98328

206-681-3627

Bjornstad, David J.

Energy Division

Oak Ridge National Laboratory

P.O. Box 2008

Oak Ridge, TN 37831

615-574-5152

Boden, Thomas A.

Carbon Dioxide Information Analysis Center Oak Ridge National Laboratory

P.O. Box 2008

Oak Ridge, TN 37831-6335

615-574-0390

Bradley, Raymond S.

Department of Geology and Geography

University of Massachusetts

Amherst, MA 01003

413-545-2794

Broecker, Wallace S.

Lamont-Doherty Earth Observatory

Columbia University

Palisades, NY 10964

914-359-2900, E413

Brown, Sandra

Department of Forestry

W-503 Turner Hall

University of Illinois

Urbana, IL 61801

217-333-1643

Byme, R. Nelson

Science Applications International Corp.

10260 Campus Point Drive

San Diego, CA 92121

619-546-6485

Cess, Robert D.

State Univerity of New York at Stony Brook Stony Brook, NY 11794-2300

516-623-8321
Cessi, Paola

Scripps Institution of Oceanography

University of California, San Diego

San Diego, CA 92093-0230

619-534-0622

Chervin, Robert M.

National Center for Atmospheric Research

P.O. Box 3000

Boulder, CO 80307

303-497-1339

Clough, Shcpard A.

Atmospheric and Environmental Research, Inc.

840 Memorial Drive

Cambridge, MA 02139

617-547-6207

Cotton, William R.

Department of Atmospheric Sciences

Colorodo State University

Fort Collins, CO 80523

303-491-8593

Coulter, Richard L

Argonne National Laboratory

9700 S. Cass Avenue

Argonne, IL 60439

708-252-5833

Cox, Stephen K.

Department of Atmosheric Sciences

Colorado State University

Fort Collins, CO 80523

303-491-8594

Crane, Robert G.

Pennsylvania State University

308 Walker Building

University Park, PA 16802

814865-6432

Crowley, T. J.

ARC Technologies

305 Arguello Drive

College Station, TX 77840

409-846-1403

Cushman, Robert

Carbon Dioxide Information Analysis Center

Oak Ridge National Laboratory

P.O. Box 2008

Oak Ridge, TN 37831.6335

615-574-0390 
Dabberct, Walter F.

Atmospheric Technology Division

National Center for Atmospheric Research

P.O. Box 3000

Boulder, CO $80307-3000$

303-497-8819

Dannevik, William P.

Lawrence Livermore National Laboratory

P.O. Box 808

Livermore, CA 94550

415-422-3132

Daum, Peter H.

Environmental Science Division

Brookhaven National Laboratory

Upton, NY 11973

516-282-7283

Del Genio, Anthony D.

Goddard Institute for Space Studies

2880 Brosdway

New York, NY 10025

212-678-5500

Delwiche, C. C.

National Institute for Global

Environmental Change

1477 Drew Avenue

Davis, CA 95616

916-757-3350

Denbo, D. W.

Battelle Marine Sciences Laboratory 439 West Sequim Bay Road

Sequim, WA 98382

206-683-3616

Deroos, Bradley G.

Battelle Memorial Institute

505 King Avenue

Columbus, OH 43201-2693

614-424-3943

Dickinson, Robert E.

Institute of Atmospheric Physics

University of Arizona

Tucson, AZ 85721

602-621-2810

Dickson, Andrew G.

Marine Physical Laboratory

0902

Scripps Institution of Oceanography

La Jolla, CA 92093

619-534-2582
Doran, J. Christopher

Pacific Northwest Laboratory

P.O. Box 999

Richland, WA 99352

509-376-4714

Dowlatabedi, $\mathbf{H}$.

Global Climate Change Program

Carnegie-Mellon University

Pittsburgh, PA 15213

412-268-3031

Downing, John P.

Battelle Marine Sciences Laboratory

439 West Sequim Bay Road

Sequim, WA 98328

206-681-3607

Drake, Bert G.

Smithsonian Environmental Research Center

P.O. Box 28, Center Wharf Road

Edgewater, MD 21037-0028

301-261-9796

Drake, John B.

Engineering Physics and Mathematics Division

Oak Ridge National Laboratory

P.O. Box 2008

Oak Ridge, TN 37831-6367

615-574-8670

Druyan, Leonard $\mathbf{M}$.

Columbia University

2880 Broadway

New York, NY 10025

212-678-5564

Edgerton, Sylvia

National Science Foundation

Committee on Earth and Environmental Sciences

1800 G Street, NW

Washington, DC 20550

202-786-9502

Edmonds, James A.

Battelle Pacific Northwest Laboratory

370 L'enfant Promenade, Suite 900

901 D St., SW

Washington, DC 20024-2115

202-646-5243

Ellingson, Robert G.

Cooperative Institute for Climate Studies

University of Maryland

College Park, MD 20742

301-405-5386 
Emanuel, Kerry A.

Center for Meteorology and Physical Oceanography Massachusetts Institute of Technology

Cambridge, MA 02139

617-253-2462

Emanuel, W. R.

Carbon Dioxide Information Analysis Center

Oak Ridge National Laboratory

P.O. Box 2008

Oak Ridge, TN 37831-6335

615-574-0390

Emerson, Steven

School of Oceanography, WB-10

University of Washington

Seattle, WA 98195

206-543-0428

Entzminger, John $\mathbf{N}$.

Advanced Research Project Agency

3701 N. Fairfax Drive

Arlington, VA 22203-1714

703-696-2295

Falkowski, Paul G.

Oceanographic and Atmospheric Sciences Division

Brookhaven National Laboratory

Upton, NY 11973

516-282-2961

Farrell, Brian F.

Harvard University

Div. of applied Sci/Pierce Hall

Cambridge, MA 02138

617-495-2998

Fischhoff, Baruch

Department of Social and

Decision Sciences

Carnegie-Mellon University

Pittsburgh, PA 15213

412-268-3246

Foster, I. T.

Argonne National Laboratory

9700 S. Cass Ave

Argonne, IL 60439

708-252-4619

Fung, Inez Y.-S.

Goddard Institute for Space Studies

2880 Broadway

New York, NY 10025

212-678-5590
Gahn, Steven J.

Pacific Northwest Laboratory

P.O. Box 999

Richland, WA 99352

509-376-3161

Gates, W. Lawrence

Lawrence Livermore National Laboratory

P.O. Box 808

Livermore, CA 94550

415-422-7642

Gautier, Catherine

Earth-Space Research Group

University of California

5276 Hollister Ave., Suite 26

Santa Barbara, CA 93111

805-893-8095

Goldsmith, J. E. M.

Organization 8354

Sandia National Laboratories

Livermore, CA 94551-0969

415-294-2432

Goyet, Catherine

Clark 418

Woods Hole Oceanographic Institution

Woods Hole, MA 02543

508-457-2000, Ext 2552

Gray, Henry

Department of Statistical Sciences

Southern Methodist University

Dallas, TX 75275

214-768-2441

Gunst, $\mathbf{R}$.

Department of Statistical Sciences

Southern Methodist University

Dallas, TX 75275

214-692-2466

Gutowski, William J.

Department of Geological and Atmospheric Sciences Agronomy Bldg.

Iowa State University

Ames, IA 50011

515-294-5632

Hack, James J.

Climate Modeling Section

National Center for Atmospheric Research

Boulder, CO 80307-3000

303-497-1387 
Hall, Charles A. S.

State University of New York

459 Illick Hall, Rm. 350

Syracuse, NY 13210

315-470-6870

Hameed, S.

Laboratory for Planetary Atmospheres Research

State University of New York at Stony Brook

Long Island, NY 11794

516-246-8319

Hanson, Howard P.

Center for the Study of Global Change, CB 2

CIRES

University of Colorado

Boulder, CO 80309-0216

303-492-1227

Harrison, Lee

Atmospheric Sciences Research Center

State University of New York

100 Fuller Rd.

Albany, NY 12205

$518-442-3811$

Hellmer, Hartmut $\mathrm{H}$.

Lamont-Doherty Earth Observatory

Route 9W

Palisades, NY 10964

914-365-8576

Hendrey, George

Terrestrial and Aquatic Ecology

Brookhaven National Laboratory

Upton, NY 11973

516-282-3262

Heymsfield, Andrew J.

National Center for Atmospheric Research

P.O. Box 3000

Boulder, CO 80307-3000

303-497-8943

Hoffert, Martin I.

New York University

26-36 Stuyvesant St.

New York, NY 10003

212-998-8995

Hosker, R. P.

Atmospheric Turbulence and Diffusion Division/NOAA 456 South Illinois Avenue

P.O. Box 2456

Oak Ridge, TN 37831-2456

615-626-1248
Houghton, R. A.

Woods Hole Research Center

P.O. Box 296

Woods Hole, MA 02543

617-540-9900

Hudson, James G.

Atmospheric Sciences Center

Desert Research Institute

P.O. Box 60220

Reno, NV 89506-0220

702-677-3119

Huntington, H. G.

Energy Modeling Forum

Stanford University

Stanford, CA 94305

415-723-1050

Jacobs, S. S.

Lamont-Doherty Earth Observatory

Columbia University

Palisades, NY 10964

914359-2900, X-326

Jacoby, Henry

Joint Program on the Science and

Policy of Global Change

Massachusetts Institute of Technology

Cambridge, MA 02139

617-253-6609

Jensen, Tommy

Department of Atmospheric Sciences

Colorado State University

Fort Collins, CO 80532

303-491-8533

Johnson, Donald R.

Space Science and Engineering Center

University of Wisconsin

Madison, WI 53706

608-262-0783

Johnson, $\mathbf{K}$.

Oceanographic Sciences Division

Brookhaven National Laboratory

Upton, NY 11973

516-282-5668

Jones, P. D.

School of Environmental Sciences

University of East Anglia

Norwich, NR4 7TJ

U.K.

011-44-603-56161 
Kao, C. Y. Jim

Los Alamos National Laboratory

P.O. Box 1663

Los Alamos, NM 87545

505-667-9226

Karl, Thomas R.

National Climatic Data Center/NOAA

Federal Building

Asheville, NC 28801

704-271-4319

Keeling, Charles D.

2314 Ritter Hall

A-020

Scripps Institution of Oceanography

La Jolla, CA 92093

619-534-4230

Kegelmeyer, W. Philip, Jr.

Sandia National Laboratories

P.O. Box 969

Livermore, CA 94550-0969

415-294-3016

Key, R. M.

Atmospheric and Oceanic Science

Princeton University

Princeton, NJ 08540

609-258-3595

Khalil, M. A. K.

Oregon Graduate Institute

19600 NW von Neumann Drive

Beaverton, OR 97006-1999

503-690-1093

Kiehl, Jeffrey $T$.

National Center for Atinospheric Research

P.O. Box 3000

Boulder, CO $803077-3000$

303-497-1350

Kimball, Bruce

USDA-ARS Waler Conservation Laboratory

4331 E. Broadvay

Phoenix, AZ 85040

602-379-4356

King, A. W.

Carbon Dioxide Information Analysis Center

Oak Ridge National Laboratory

P.O. Box 2008

Oak Ridge, TN 37831-6335

615-574-0390
Kinter, James L.

Institute of Global Environmental and Society 4041 Powder Mill Road

Calverton, MD 20705

301-984-0639

Kleinman, Lawrence I.

Environmental Chemistry Division

Bldg. 426

Brookhaven National Laboratory

Upton, NY 11973

516-282-3796

Kliewer, Kenneth L.

Oak Ridge National Laboratory

P.O. Box 2008

Oak Ridge, TN 37831-6367

615-241-2859

Ko, Malcolm K. W.

Atmospheric and Environmental Research, Inc.

840 Memorial Drive

Cambridge, MA 02139

617-547-6207

Kropfli, Robert A.

ERL/NOAA Wave Propagation Laboratory

325 Broadway

Boulder, CO 80303

303-497-6235

Kukla, George

Lamont-Doherty Earth Observatory

Columbia University

Palisades, NY 10964

914359-2900

Kulp, Thomas J.

Lawrence Livermore National Laboratory

P.O. Box 808

Livermore, CA 94550

510-423-7839

Kunkel, Kenneth

Illinois State Water Survey

2204 Griffith Dr.

Champaign, Il 61820

217-244-1488

Kutzbach, John E.

Center for Climatic Research

University of Wisconsin

1225 West Dayton St.

Madison, WI 53706

608-262-2839 
Lacis, Andrew A.

Nasa Goddard Institute for Space Studies

2880 Broadway

New York, NY 10025

212-678-5595

Lambert, Richard

National Science Foundation

18 ih and G St., NW

Washington, DC 20550

202-357-9614

Lapp, $\mathbf{M}$.

Organization 8300A

Sandia National Laboratory

Livermore, CA 94551-0969

415-294-2435

Laulainen, Nels $\mathbf{S}$.

Pacific Northwest Laboratory, MS K6-84

P.O. Box 999

Richland, WA 99352

509-376-4155

Lave, Lester B.

Graduate School of Industrial Administration

Carnegie-Mellon University

Schenley Park

Pittsburgh, PA 15213

412-268-8837

Leiby, Paul N.

Energy Division

Oak Ridge National Laboratory

P.O. Box 2008

Oak Ridge, TN 37831

615-574-7720

Leifer, Robert

Environmental Measurements Laboratory

376 Hudson Street

New York, NY 10014-3621

212-620-3626

Leone, John, Jr.

Atmospheric and Geophysics Division

Lawrence Livermore National Laboratory

P.O. Box 808

Livermore, CA 94550

415-422-6449

Lincoln, David E.

Department of Biology

University of South Carolina

Columbia, SC 29:208

803-777-7306
Lindzen, Richard S.

Massachusetts Institute of Technology

Room 54-1720

Cambridge, MA 02139

617-253-2432

Louis, Jean-Francois

Atmospheric and Environmental Research, Inc.

840 Memorial Drive

Cambridge, MA 02139

617-547-6207

Luxmoore, Robert J.

Environmental Sciences Division

Oak Ridge National Laboratory

P.O. Box 2008

Oak Ridge, TN 37831-6038

615-574-7357

MacCracken, Michael C.

Lawrence Livermore National Laboratory

P.O. Box 808

Livermore, CA 94550

415-422-1826

MacKenzie, F. T.

Department of Oceanography

University of Hawaii

100 Pope Rd.

MSB-525

Honolulu, HI 96822

808-956-6344

Malone, Robert C.

Los Alamos National Laboratory

P.O. Box 1663

Los Alamos, NM 87545

505-667-5925

Margolin, Len G.

MS B-284

Los Alamos National Laboratory

P.O. Box 1663

Los Alamos, NM 87545

505-667-4843

Marotzke, J.

Massachusetts Institute of Technology

Cambridge, MA 02139

617-253-5939

McCurdy, W.

Lawrence Livermore National Laboratory

P.O. Box 808

Livermore, CA 94550

$510-422-6383$ 
McIntosh, Robert E.

Department of Electrical and Computer Engineering University of Massachusetts

Amherst, MA 01003

413-545-4858

Mechoso, C. R.

Department of Atmospheric Sciences

University of California at Los Angeles

Los Angeles, CA 90024-1565

310-825-3057

Meehl, Gerald A.

National Center for Atmospheric Research

P.O. Box 3000

Boulder, CO 80307

303-497-1331

Meier, Mark F.

INSTAAR, Campus Box 450

University of Colorado

Boulder, CO 80309

303-492-7909

Melfi, Harvey

Mail Code 617

NASA Goddard Spaceflight Center

Greenbelt Rd.

Greenbelt, MD 20771

301-286-6348

Michalsky, Joseph

Atmospheric Science Research Center

State University of New York at Albany

Albany, NY 12222

518-442-3809

Miller, A.

A-024

Climate Research Division

Scripps Institution of Oceanography

La Jolla, CA 92093-0221

$619-534-5649$

Millero, Frank J.

University of Miami

4600 Rickenbacker Causeway

Miami, FL 33149-1098

305-361-4731

Mirin, A. A.

Lawrence Livermore National Laboratory

P.O. Box 808

Livermore, CA 94550

$510-422-4020$
Mconey, Harold A.

Department of Biological Sciences

Stanford University

Stanford, CA 94305-5020

415-723-1179

Moore, Barrien, III

Complex Systems Research Center

University of New Hampshire

Durham, NH 03824

603-862-1792

Murcray, David G.

Department of Physics

University of Denver

Denver, CO 80208

303-871-2627

Nakicenovic, Nebojsa

International Institute for Applied Systems Analysis

Schlossplatz 1

A-2361 Laxenburg, Austria

432236715210

Nobel, Park S.

Laboratory of Biomedical and Environmental Sciences

University of California at Los Angeles

900 Veterans Ave

Los Angeles, CA 90024

213-825-3395

Norby, Richard J,

Environmental Sciences Division

Oak Ridge National Laboratory

P.O. Box 2008

Oak Ridge, TN 37831-6034

615-574-7301

North, G. R.

ARC Technologies

305 Arguello Dr.

College Station, TX 77840

409-846-1403

North, Gerald R.

Department of Meteorology

Texas A\&M University

College Station, TX 77843-3146

409-845-8083

Novakov, Tihomir

1 Cyclotron Road

Lawrence Berkeley Laboratory

Berkeley, CA 94720

510-486-5319 
Nowlin, Worth D.

U.S. WOCE Office

Texas A\&M Research Foundation

Box 3578

College Station, TX 77843

409-845-3720

Oechel, Walter C.

Department of Biology

San Diego State University

San Diego, CA 92182

714-265-6767

Oglesby, Robert J.

Department of Geological Sciences

Brown University

Providence, RI 02912

401-863-3626

Ohring, George

NOAA/NESDIS, E/RA1

Washington, DC 20233

301-763-8078

O'Neill, E. G.

Environmental Sciences Division

Oak Ridge National Laboratory

P.O. Box 2008

Oak Ridge, TN 37831-6038

615-574-7850

Owensby, Clenton E.

Department of Agronomy

Throckmorton Hall

Kansas State University

Manhattan, KS 66506-5501

913-532-5731

Paluszkiewicz, Theresa

Battelle Marine Sciences Laboratory

439 West Sequim Bay Road

Sequim, WA 98232

206-683-3667

Peng, T.-H.

Environmental Sciences Division

Oak Ridge National Laboratory

P.O. Box 2008

Oak Ridge, TN 37831-6335

615-574-0390

Penner, Joyce E.

Lawrence Livermore National Laboratory

P.O. Box 808

Livermore, CA 94550

415-422-4140
Pfeffer, W. Tad

INSTAAR, Campus Box 450

University of Colorado

Boulder, CO 80309

303-492-3480

Piacsek, Steve A.

Ocean Sciences Division

Naval Research Laboratory

Stennis Space Center, MS 39529

601-688-5316

Platt, C. M. R.

Division of Atmospheric Research

CSIRO

Private Mail Bag No. 1

Mordiallac, Vic. 3195, Australia

011-61-03-5867665

Ponch, William B.

Los Alamos National Laboratory

P.O. Box 1663

Los Alamos, NM 87545

505-667-0971

Post, W. M.

Carbon Dioxide Information Analysis Center

Oak Ridge National Laboratory

P.O. Box 2008

Oak Ridge, TN 37831-6335

615-574-0390

Preller, R. H.

Ocean Sciences Division

Naval Research Laboratory

Stennis Space Center, MS 39529

601-688-5444

Prinn, Ronald G.

Room 54-1312

Joint Program on the Science and Policy of Global

Massachusetts Institute of Technology

Cambridge, MA 02139

617-253-2452

Ramanathan, V.

Scripps Institution of Oceanography

California Space Institute

La Jolla, CA 92093-0239

619-534-8114

Randall, David A.

Department of Atmospheric Physics

Colorado State University

Ft. Collins, CO 80523

303-491-8474 
Rasmussen, R. A.

Global Change Research Center

Oregon Graduate Institute

Portland, OR 97291-1000

503-690-1093

Revercomb, Henry E.

Space Science and Engineering Center

University of Wisconsin

Madison, WI 53706

608-263-6758

Reynolds, James F.

Department of Botany

Duke University

Box 90340

Durham, NC 27706

919-684-5485

Richards, John F.

Department of History

Duke University

Durham, NC 27706

919-684-3626

Robock, Alan

Department of Meteorology

University of Maryland

College Park, MD 20742

301-405-5377

Rouhi, Safiali

0402

Institute for Nonlinear Science

University of California at San Diego

La Jolla, CA 92093-0402

619-534-1675

Sarmiento, Jorge L.

Atmospheric and Oceanic Science

Princeton University

Princeton, NJ 08540

609-452-6585

Sassen, Kenneth

819 Wm. Browning Bldg.

University of Utah

Salt Lake City, UT 84122

801-581-6136

Schimel, David

National Center for Atmospheric Research

P.O. Box 3000

Boulder, CO 80307

303-497-1610
Schlesinger, Michael E.

Department of Atmospheric Sciences

University of Illinois

105 S. Gregory Ave.

Urbana, IL 61801

217-333-2192

Semtner, Albert J.

Department of Oceanography

Naval Postgraduate School

Monterey, CA 93943

408-646-3267

Siegel, Donald I.

204 Heroy Geology Laboratory

Syracuse University

Syracuse, NY 13244-1070

315-443-2672

Siegenthaler, U.

Physikalisches Institut

University of Bern

Sidlerstrasse 5, CH3012

Bern, Switzerland

011-41-31-658611

Simpson, Marc L.

Oak Ridge National Laboratory

P.O. Box 2008

Oak Ridge, TN 37831

615-574-4171

Skole, David J.

Complex Systems Research Center

University of New Hampshire

Durham, NH 03824

603-862-1792

Skyllingstad, E. D.

Battelle Marine Sciences Laboratory

439 West Sequim Bay Road

Sequim, WA 98328

206-681-3617

Small, $H$.

Civil Engineering/Engineering and Public Policy

Carnegie-Mellon University

Pittsburgh, PA 15213

412-268-3031

Smith, Richard D.

Los Alamos National Laboratory, MS B-216

P.O. Box 1663

Los Alamos, NM 87545

505-667-7744 
Smith, William L.

Cooperative Institute for

Meteorological Satellite Studies

University of Wisconsin

Madison, WI 53706

608-262-0544

Somerville, Richard C. J.

Scripps Institution of Oceanography

La Jolla, CA 92093

619-534-4644

South, D.

Argonne National Laboratory

9600 S. Cass Ave.

Argonne, IL

708-252-7353

Spaeth, Warren $T$.

Pacific Northwest Laboratory

P.O. Box 999

Richland, WA 99352

202-646-5246

Spinhirne, James

Goddard Space Flight Center/917

Greenbelt, MD 20771

301-286-9099

Spratlin, T. L.

Oak Ridge National Laboratory

P.O. Box 2008

Oak Ridge, TN 37831

615-574-5619

Sprigg, William

National Academy of Sciences

2101 Constitution Ave., NW

Washington, DC 20418

202-334-3515

Stamnes, Knut

Geophysical Institute

University of Alaska

Fairbanks, AK 99775-0800

907-474-7368

Stephens, Graeme L.

Department of Atmospheric Sciences

Colorado State University

Fort Collins, CO 80523

303-491-8541
Stewart, H. Bruce

Department of Applied Science

Brookhaven National Laboratory

Upton, NY 11973

516-282-4179

Stokes, Gerald M.

Pacific Northwest Laboratory

P.O. Box 999

Richland, WA 99352

509-375-3816

Stone, Peter H.

Room 54-1718

Massachusetts Institute of Technology

Cambridge, MA 02139

617-253-2443

Strain, Boyd R.

Department of Botany

Duke University

Durham, NC 27706

919-684-6523

Stull, Roland B.

Department of Meteorology

University of Wisconsin

1225 West Dayton St.

Madison, WI 53706

608-262-9862

Swisshelm, Julie

Sandia National Laboratory

P.O. Box 5800

Albuquerque, NM 87123

505-845-7999

Takahashi, Taro

Lamont-Doherty Earth Observatory

Columbia University

Palisades, NY 10964

914-359-2900, 537

Toman, Michael A.

Resources for the Future

1616 P Street, NW

Washington, DC 20036

202-328-5091

Toon, Owen B.

MS 245-3

Earth System Science Division

NASA Ames Research Center

Moffett Field, CA 94035-1000

415-604-5971 
Uhlir, Paul F.

National Academy of Sciences

2101 Constitution Ave., NW

Washington, DC 20418

202-334-3061

Walcek, Chris J.

Atmospheric Sciences Research venter

State University of New York at Albany

1400 Washington Ave.

Albany, NY 12205

$518-442-3840$

Wallace, Douglas W. R.

Oceanographic Sciences Division, Bldg. 318

Brookhaven National Laboratory

Upton, NY 11973

516-282-2945

Wang, Wei-Chyung

Atmospheric Sciences Research Center

State University of New York at Albany

Albany, NY 12222

518-442-3357

Washington, Warren $\mathbf{M}$.

National Center for Atmospheric Research P.O. Box 3000

Boulder, CO 80307-3000

303-497-1321

Webb, Thompson, III

Department of Geological Sciences

Brown University

Providence, RI 02912-1846

401-863-3334

Weiss, R. F.

A.020

Scripps Institution of Oceanography

La Jolla, CA 92093

619-534-2598

Westphal, D.

MS 245-5

NASA Ames Research Center

Mountain View, CA 94035-1000

415-604-3522

Weyant, John P.

Stanford University

Engineering-Economic Systems

Stanford, CA 94305

415-723-3506
White, Andrew B.

Los Alamos National Laboratory, MS B-260

P.O. Box 1663

Los Alamos, NM 87545

505-667-4530

Whiteman, Charles D.

Pacific Northwest Laboratory

P.O. Box 999, KG-11

Richland, WA 99352

509-376-7859

Wigley, T. M. L.

School of Environmental Sciences

University of East Anglia

Norwich, NR4 7TJ United Kingdom

011-44-603-56161

Williams, Allen L.

Illinois State Water Survey

2204 Griffith Dr.

Champaign, IL 61820

217-244-0373

Williams, Edward R.

DOE

EP-60

Washington, D.C. 20585

202-586-2061

Winn, Christopher D.

University of Hawaii

1000 Pope Road, MSB 610

Honolulu, HI 96822

808-956-7625

Wohlpart, A

Oak Ridge Institute for Science

and Education

P.O. Box 117

Oak Ridge, TN 37831-0117

615-576-3421

Woodward, Wayne A.

Department of Statistical Sciences

Southern Methodist University

Dallas, TX 75275

214-692-2457

Wuebbles, Donald J.

Lawrence Livermore National Laboratory

P.O. Box 808

Livermore, CA 94550

415-422-1845 
Yang, Zili

Oak Ridge National Laboratory

P.O. Box 2008

Oak Ridge, TN 37831 


\section{APPENDIX B: ACRONYMS}

$\begin{array}{ll}\text { 1-D } & \text { One-dimensional } \\ \text { 2-D } & \text { Two-dimensional } \\ \text { 3-D } & \text { Three-dimensional } \\ \text { 4-D } & \text { Four-dimensional } \\ \text { ACC } & \text { Antarctic Circumpolar Current } \\ \text { ACM } & \text { Advanced climate model } \\ \text { AEEI } & \text { Autonomous energy-efficiency index } \\ \text { AERI } & \text { Atmospheric emitted radiance instrument } \\ \text { AGCM } & \text { Atmospheric general circulation model } \\ \text { AIM } & \text { Approximate inertial manifold } \\ \text { ALK } & \text { Alkalinity } \\ \text { AMIP } & \text { Atmospheric Model Intercomparison Project } \\ \text { ANL } & \text { Argonne National Laboratory } \\ \text { ARCS } & \text { Atmospheric radiation and cloud station } \\ \text { ARCSS } & \text { Arctic System Science Program } \\ \text { ARM } & \text { Atmospheric Radiation Measurement (Program) } \\ \text { ARMA } & \text { Autoregressive moving-average } \\ \text { ARS } & \text { Agricultural Research Service } \\ \text { ASL } & \text { Atmospheric surface layer } \\ \text { ASTEX } & \text { Atlantic Stratocumulus Transition Experiment } \\ \text { ATPEX } & \text { ARM Tropical Pacific Experiment } \\ \text { AVHRR } & \text { Advanced very-high-resolution radiometer } \\ \text { AXCTD } & \text { Autonomous expendable CTD profiler } \\ \text { BNL } & \text { Brookhaven National Laboratory } \\ \text { BVE } & \text { Barotropic vorticity equation } \\ \text { CAC } & \text { Committee on Atmospheric Chemistry } \\ \text { CAM } & \text { Crassulacean acid metabolism } \\ \text { CARDS } & \text { Climatological aerological reference data set } \\ \text { CART } & \text { Clouds and Radiation Testbed } \\ \text { CCCO } & \text { Committee on Climatic Change and the Ocean } \\ \text { CCD } & \text { Charged couplel device } \\ \text { CCM } & \text { Community climate model } \\ \text { CCN } & \text { Cloud condensation nuclei } \\ \text { CD-ROM } & \text { Compact disk-read only memory } \\ \text { CDIAC } & \text { Carbon Dioxide Information Analysis Center } \\ \text { CE } & \text { Office of Conservation and Renewable Energy } \\ \text { CEA } & \text { Cause-and-effect analysis } \\ \text { CEES } & \text { Committee on Earth and Environmental Sciences } \\ \text { CEM } & \text { Cumulus ensemble model } \\ \text { CEPEX } & \text { Central Equatorial Pacific Experiment } \\ \text { CFC } & \text { Chlorofluorocarbon } \\ \text { CHAMMP } & \text { Compdvanced Mathematics, and Model Physics } \\ \text { CIS } & \end{array}$




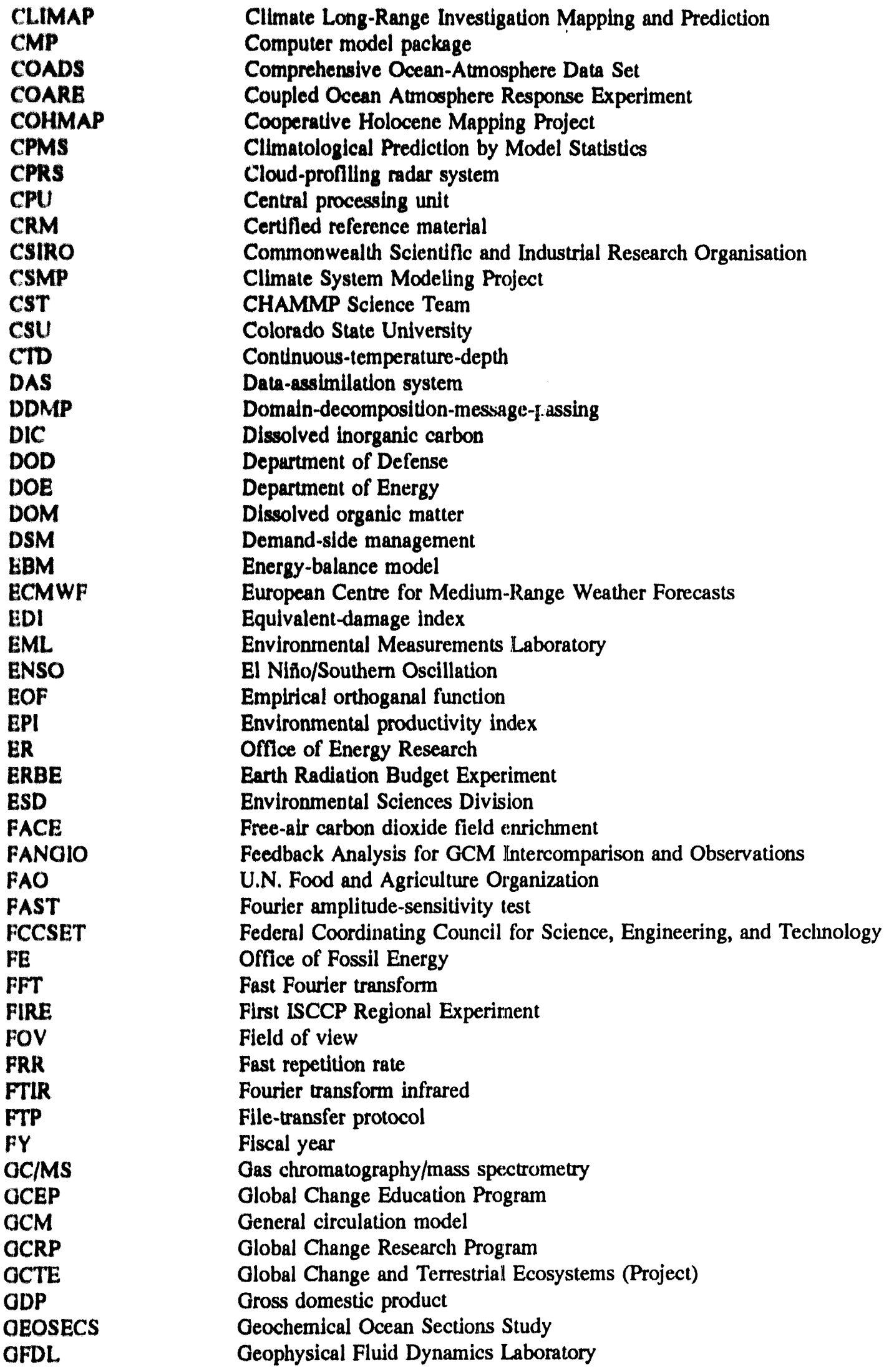




\begin{tabular}{|c|c|}
\hline GGI & Greenhouse-gas indicies \\
\hline GHCN & Global Historical Climatology Network \\
\hline GIS & Geographic information system \\
\hline GISMO & Graphic-information-system modeling \\
\hline GISS & Goddard Institute for Space Studies \\
\hline GRASS & Geographic Resource Analysis Support System \\
\hline GTS & Global Telecommunications System \\
\hline GWP & Global warming potential \\
\hline HAPEX & Hydrologic Atmospheric Pilot Experiment \\
\hline HPCRC & High-Performance Computing Research Center \\
\hline HPLC & High-pressure liquid chromatography \\
\hline I/O & Input/output \\
\hline IBP & International Biophysical Program \\
\hline IC & Interaction coefficient \\
\hline ICRCCM & InterComparison of Radiation Codes in Climate Models (Program) \\
\hline IDP & Instrument Development Program \\
\hline IEM & Institute of Experimental Meteorology \\
\hline IGBP & International Geosphere-Biosphere Program \\
\hline IOP & Intensive operational period \\
\hline IPCC/RSWG/EIS & Response Strategies Working Group, Energy and Industry Subgroup \\
\hline IPCC & Intergovernmental Panel on Climate Change \\
\hline IR & Infrared \\
\hline ISCCP & International Satellite Cloud Climatology Project \\
\hline ISS & Integrated sounding system \\
\hline ISTAP & International Space Technology Assessment Program \\
\hline JGOFS & Joint Global Ocean Flux Study \\
\hline JJA & June-July-August \\
\hline LAID & Land-atmosphere interactions dynamics \\
\hline LANL & Los Alamos National Laboratory \\
\hline LBL (lbl) & Line by line \\
\hline LCL & Lifting condensation level \\
\hline LDEO & Lamont-Doherty Earth Observatory \\
\hline LIDAR & Laser-radar \\
\hline LLNL & Lawrence Livermore National Laboratory \\
\hline LPDA & Laser-phased Doppler anemometer \\
\hline LTER & Long-term ecological research \\
\hline MCC & Mesoscale convective complex \\
\hline MFRSR & Multifilter rotating shadowband radiometer \\
\hline MIMD & Multiple-instruction, multiple-data \\
\hline MINK & Missouri-Iowa-Nebraska-Kansas \\
\hline MM4 & Mesoscale model \\
\hline MOM & Modular ocean model \\
\hline MPC & Massively parallel computer \\
\hline MPI & Max Planck Institute \\
\hline MPL & Micropulse lidar \\
\hline MPL & Micropulse lidar \\
\hline MSU & Microwave sounding unit \\
\hline NADW & North Atlantic deep water \\
\hline NAS & National Academy of Sciences \\
\hline NASA & National Aeronautics and Space Administration \\
\hline NATO & North Atlantic Treaty Organization \\
\hline NCAR & National Center for Atmospheric $R$ \\
\hline
\end{tabular}




\begin{tabular}{|c|c|}
\hline NCDC & National Climatic Data Center \\
\hline NDP & Numeric data package \\
\hline NE & Office of Nuclear Energy \\
\hline NES & National Energy Strategy \\
\hline nf-EBM & Noise-forced energy-balance model \\
\hline NIGEC & National Institute of Global Environmental Change \\
\hline NIST & National Institute of Standards and Technology \\
\hline NMC & National Meteorological Center \\
\hline NMHC & Nonmethane hydrocarbon \\
\hline NOAA & National Oceanic and Atmospheric Administration \\
\hline NRL & Naval Research Laboratory \\
\hline NRP & National Research Program \\
\hline INRPGC & National Research Program for Global Change \\
\hline NSA & Northern Slope of Alaska \\
\hline NSF & National Science Foundation \\
\hline nss & Non-sea-salt \\
\hline NWS & National Weather Service \\
\hline NYU & New York University \\
\hline OGCM & Ocean general circulation model \\
\hline OHER & Office of Health and Environmental Research \\
\hline OMB & Office of Management and Budget \\
\hline OMWOG & Ocean Measurements Working Group \\
\hline ORNL & Oak Ridge National Laboratory \\
\hline OSB & Ocean Studies Board \\
\hline OSC & Office of Scientific Computing (DOE) \\
\hline OSSE & Observing Simulation System Experiment \\
\hline OSU & Oregon State University \\
\hline PBL & Planetary boundary layer \\
\hline PCMDI & Program for Climate Model Diagnosis and Intercomparison \\
\hline PDA & Phased-doppler anemometer \\
\hline PDF & Probability density function \\
\hline PDL & Polarization-diversity lidar \\
\hline PDS & Photothermal deflection spectroscopy \\
\hline PE & Office of Policy, Planning, and Analysis \\
\hline PEPCase & Phosphenol-pyruvate carboxylase \\
\hline PER & Program for Ecological Research \\
\hline PIC & Particle in cell \\
\hline PMIP & Paleoclimate Modeling Intercomparison Project \\
\hline PNL & Pacific Northwest National Laboratory \\
\hline POCM & Parallel ocean-climate model \\
\hline POM & Particulate organic matter \\
\hline PROBE & Pilot Radiation Observation Experiment \\
\hline PSC & Polar stratospheric clouds \\
\hline QBO & Quasi-Biennial Oscillation \\
\hline QLN & Quantitative Links Network \\
\hline QTO & Quasi-Triennial Oscillation \\
\hline RAMS & Regional Atmospheric Modeling System \\
\hline RASS & Radio acoustic sounding system \\
\hline RCM & Radiative-convective model \\
\hline RCRE & Required collaborative research experience \\
\hline RGI & Regional-global interactions \\
\hline RISC & Reduced instruction set chip \\
\hline
\end{tabular}




\begin{tabular}{|c|c|}
\hline $\mathbf{R M}$ & Reference material \\
\hline RSR & Rotating shadowband radiometer \\
\hline RT & Radiative transfer \\
\hline Rubisco & Ribulose-1,5-bisphosphate carboxylase \\
\hline SAGE & Stratospheric Aerosol and Gas Experiment \\
\hline SAT & Surface air temperature \\
\hline SAVE & South Atlantic Ventilation Experiment \\
\hline SBIR & Small Business Innovation Research \\
\hline SCAR & Scientific Committee on Antarctic Research \\
\hline SDSC & San Diego Supercomputer Center \\
\hline SERDP & Strategic Environmental Research and Development Program \\
\hline SGCR & Subcommittee on Global Change Research \\
\hline SGM & Second-generation model \\
\hline SGP & SSuthern Great Plains \\
\hline SGP & Southern Great Plains \\
\hline SIMD & Single-instruction, multiple-data \\
\hline SIO & Scripps Institution of Oceanography \\
\hline SLP & Sea-level pressure \\
\hline SOC & Soil organic carbon \\
\hline SOCEX & Southern Ocean Cloud Experiment \\
\hline SOMMA & Single-operator multiparameter metabolic analyzer \\
\hline SORTI & Solar-radiance-transmission interferometer \\
\hline SPECTRE & Spectral Radiance Experiment \\
\hline SSA & Singular spectrum analysis \\
\hline SSM/I & Special sensor microwave/imager \\
\hline SST & Sea-surface temperature \\
\hline SWE & Shallow-water equations \\
\hline SWIR & Short-wavelength infrared \\
\hline TECO & Terrestrial ecosystems \\
\hline TM & Thematic Mapper \\
\hline TOF & Theoretical orthogonal function \\
\hline TOGA & Tropical Ocean Global Atmosphere \\
\hline TOMS & Total Ozone Measuring Satellite \\
\hline TRMM & Tropical Rainfall Measuring Mission \\
\hline TTO & Transient Tracers in the Ocean \\
\hline TWPO & Tropical Western Pacific Ocean \\
\hline UAV & Unmanned aerospace vehicle \\
\hline UC & University of California \\
\hline UCAR & University Corporation for Atmospheric Research \\
\hline UCLA & University of California at Los Angeles \\
\hline UCR & University of California at Riverside \\
\hline UNCED & United Nations Conference on the Environment and Development \\
\hline USDA & United States Department of Agriculture \\
\hline USGCRP & U.S. Global Change Research Program \\
\hline USGS & United States Geological Survey \\
\hline WCRP & World Climate Research Program \\
\hline WG & Working group \\
\hline WGGC & Working Group on Global Change \\
\hline WGNE & Working Group for Numerical Experimentation \\
\hline WILASS & Water Infiltration in Layered Subfreezing Snow (Project) \\
\hline WMO & World Meteorological Organization \\
\hline WOCE & World Ocean Circulation Experiment \\
\hline
\end{tabular}


WOCE/HP

WPL

WSI

WST
World Ocean Circulation Experiment Hydrographic Program NOAA Wave Propagation Laboratory

Whole-sky imager

Whitecap simulation tank 


\section{SUBJECT INDEX}

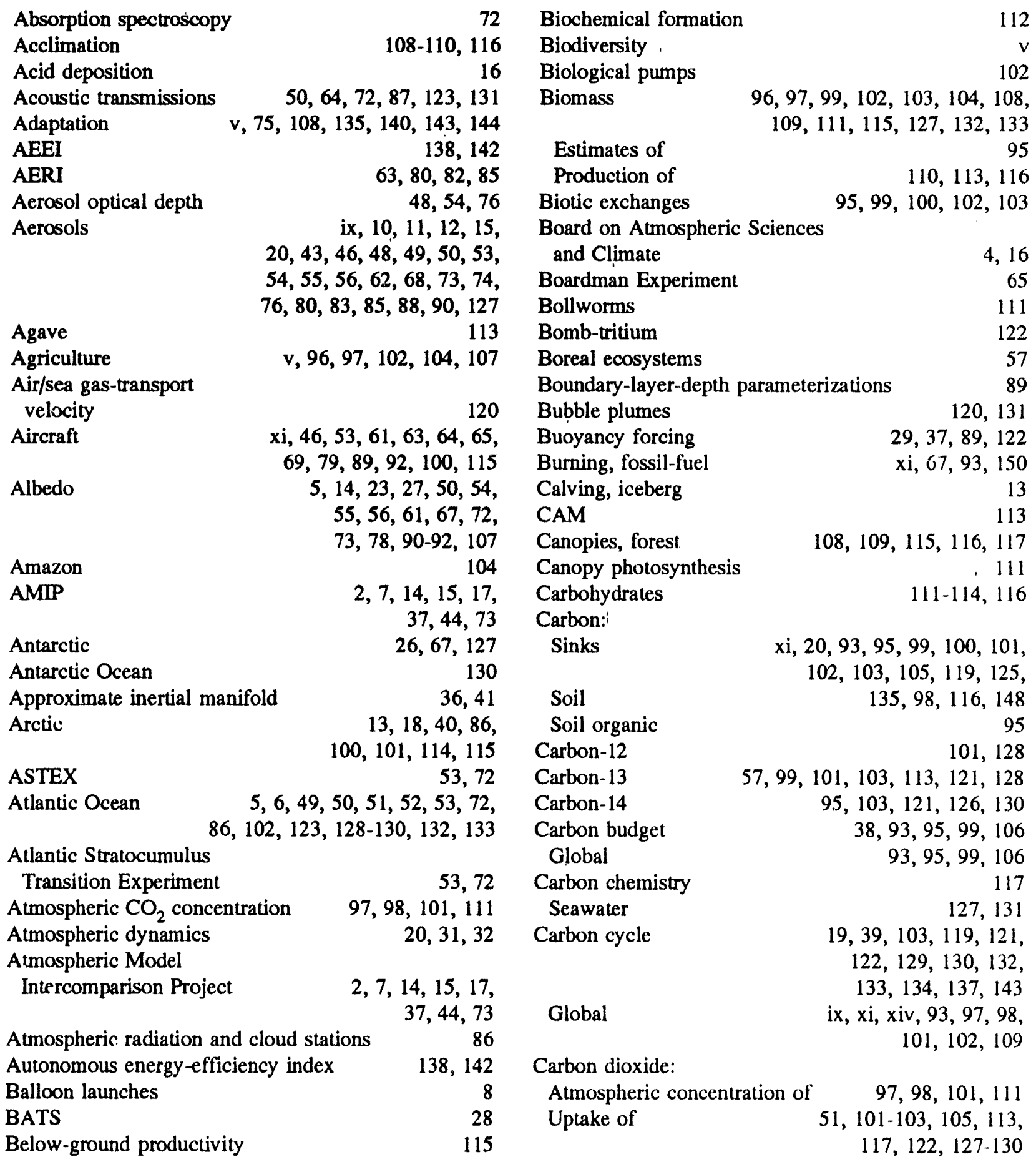




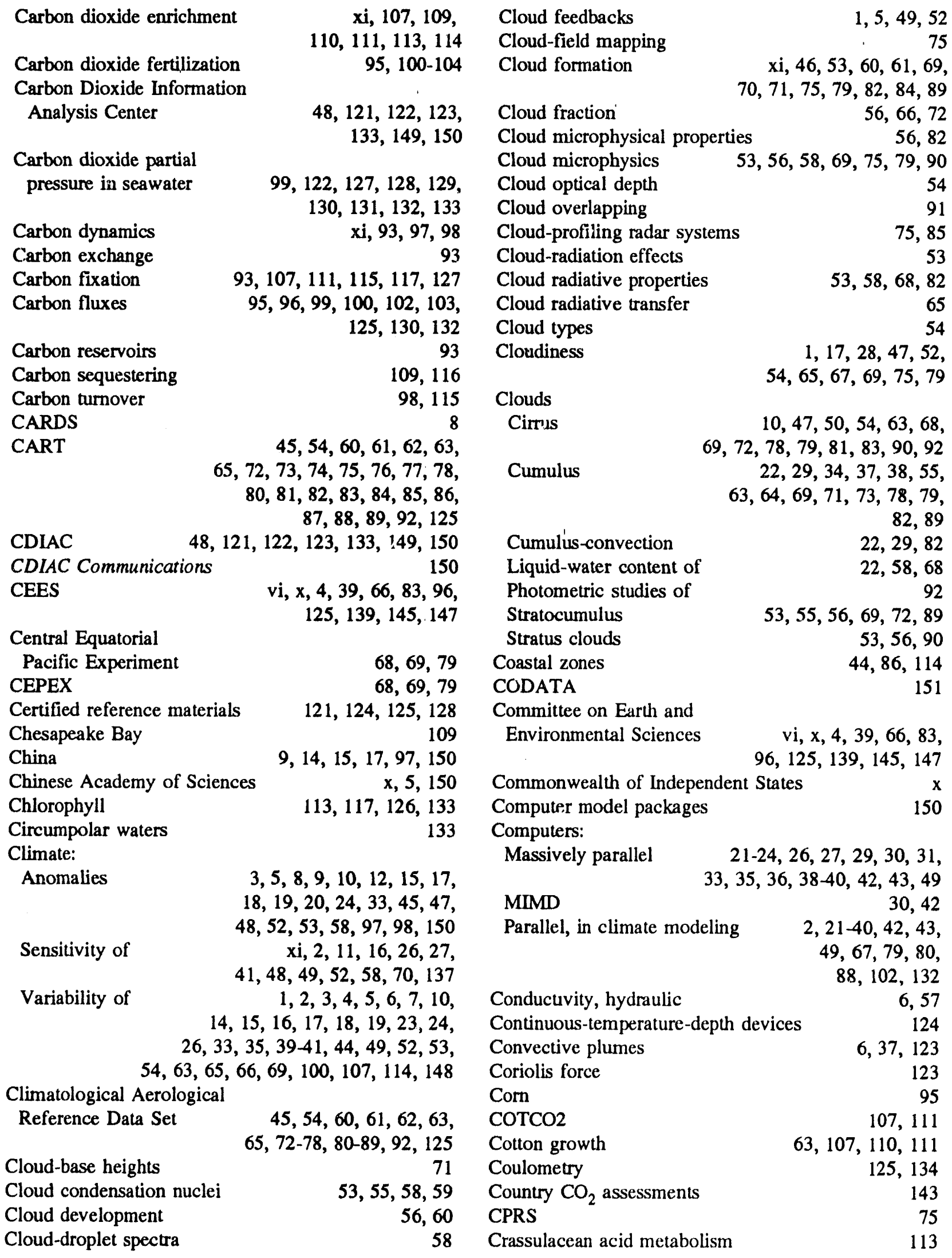




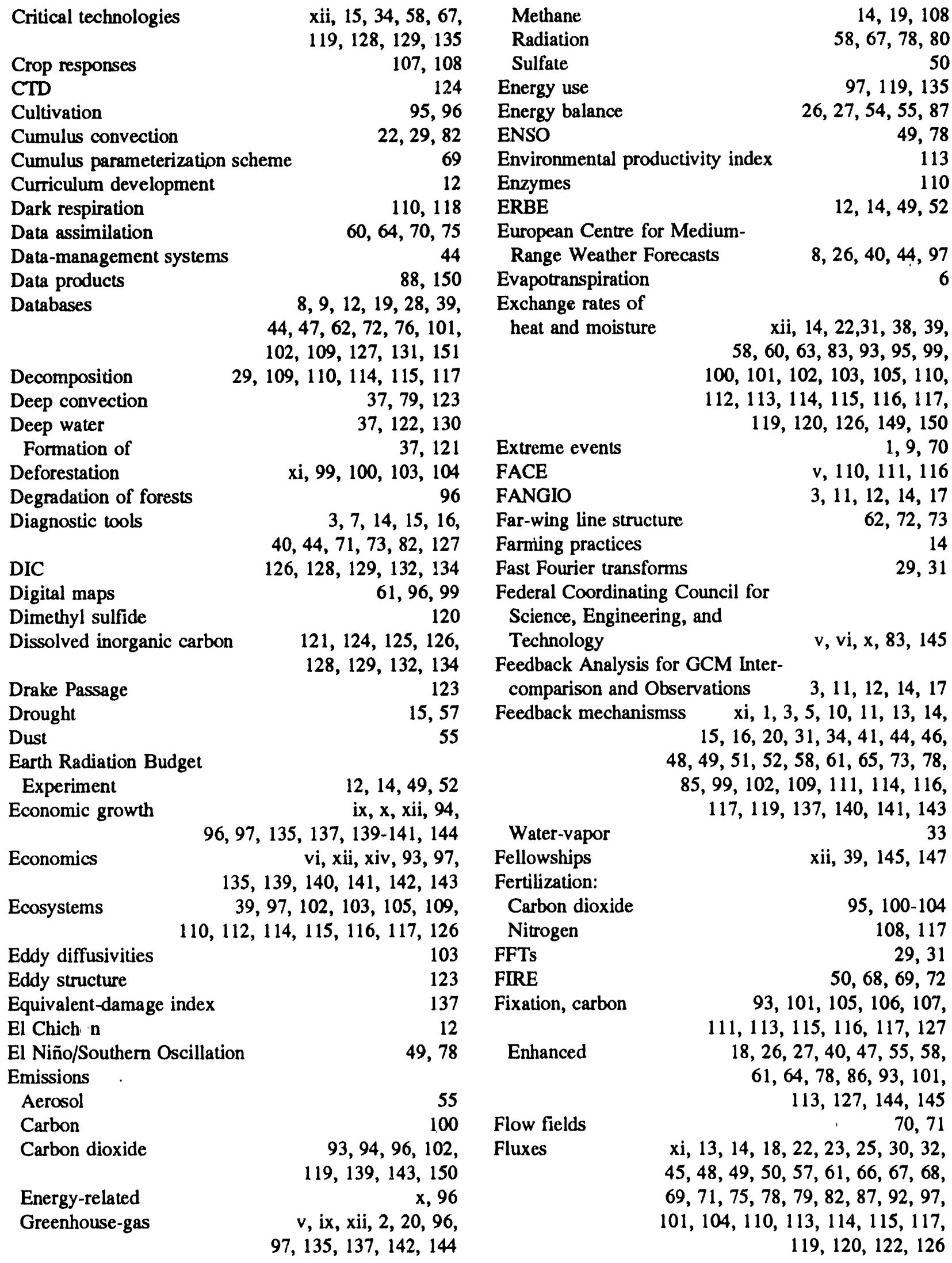




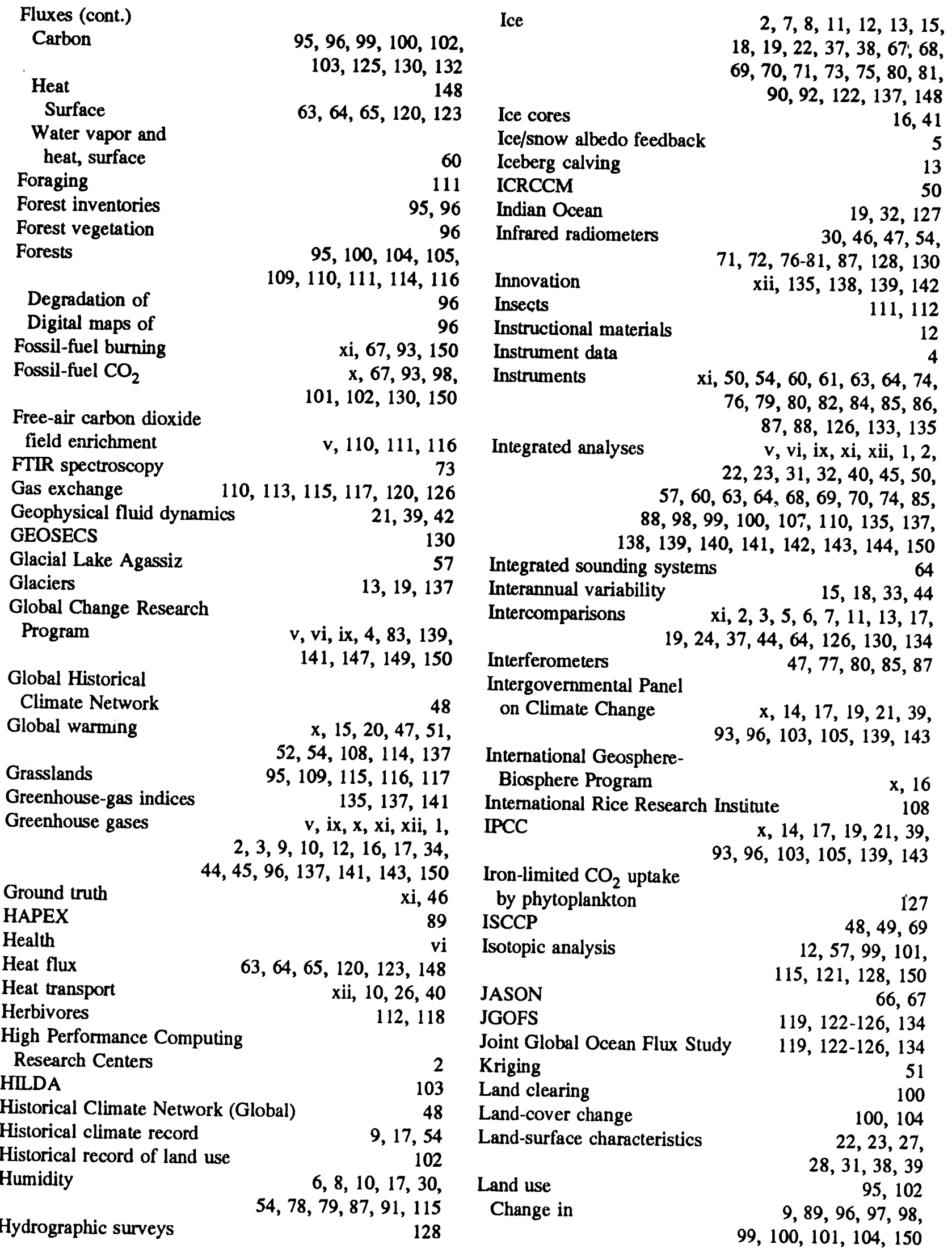


Land use (cont.)

Historical record of $\quad 102$

Larval growth

Laser-phased Doppler anemometer

Latent climatic effects

Leaf-area index

Leaf production

Lidar

Linda

Line-by-line computations

Line structure, far-wing

Litter

Logging

Long-memory processes

Maps, digital

Massively parallel computers

$48,61,63,64,68,72-74$, $77,78,80,81,83,85,87,90$

120

$6,50,55,62$ $66,73,80,82,85$ $62,72,73$ $110,114,116,117$

$21,22,23,24$,
Box 25 $26,27,29,30,31,33$, $35,36,38,39,40,42,43,49$

Mauna Loa

Mesoscale convective systems

Meteorology

Methane $92,108,114,115,133,137,150$

Methane budget

MFRSR

Micropulse lidar

Mitigation

$\mathrm{v}, \mathrm{ix}, 93,105,135$,

Mixed-layer deepening and retreat

Model development

Modeling

Bottom/up

Top/down

Models:

1-D

2-D

3-D

4-D

Advanced climate model

$56,60,64,70,74,78,79,80$,

$90,99,101,102,103,116,123,131$

$20,30,31,37,58,75$,

$79,82,89,90,91,116$

$6,7,10,20,21,25,31,41$,

$43,73,91,104,107,122,123$

$8,11,20,36,37,40,41,43$,

v, $x i, 2,8,17,18$
$27,29,30,34,35$

$21,27,29,30,34,35$,

$119,125,129,130,132-134,147$

AGCM

ALFA

Arakawa-Schubert

Baroclinic-wave
Carbon-cycle

CCM

CCM1

CCM2

CENTURY

Chervin-Semtner

Cloud microphysical

Crop

Deep-convection

Edmonds-Reilly

FASCOD3

FASCODE

Finite-difference

General circulation models
CANDO 101

$14,16,148$

14

$54,76,85$

83

$140,143,144$

34,35

60,90

$15,22,28,34$

$98,103,119,129,130,134$

$49,61,71,91$

$10,17,19,68$

$18,28-30,49,71$

$5,9,24,27,37,115,130$

$13,18,26,37,40$ $29,56,60,65$,

$68,69,81,82,90$

107

123

138

62,80

82

22,38

xi, 1, 3, 5, 6, 7,

9-15, 17-19, 21-29, 31-35,

$37-40,42,43-46,48,49,50$,

$52,54,55,58,60-63,65-71$,

$73,75,76,77,78,79,82,84$, $89-92,102,103,107,119,121$

GENLEAF

Geophysical

116

GFDL

GISS

GLYCIM

HITRAN

Intercomparison of

Isopycnal-coordinate

ocean-circulation

Mesoscale

Mixed-layer

Modular-ocean

MOM

NCAR

$22,27,71$

$6,28,41,49,73,99$

$62,72,82,92$

$\mathrm{xi}, 2,3,5,6,7,11$,

$13,17,19,24,37,44$,

$64,126,130,134$

$11,24,32,35,36,121$

$21,60,61,68,70$

52,126

$22,27,34$

$22,27,34$

Nonhydrostatic ocean

Plant-simulation

PRECO

Radiative-convective

Radiative-transfer

Sea-ice model

Semtner-Chervin

Sensitivity of

$49,55,61,64,71,92,145$

$21,64,68,69$

108

117

58,91

$60,61,62,66,90,92,116$

$12,15,38$

$13,18,26,37,40$

$10,16,18-20,22,26,27$,

$28,30,33,37,38,44,49,61$,

SERECO

$66,68,71,75,82,98,99,103,107$

SOILSIM

116,117

20, 22, 69

$24,30,33,34$

SPECTRE
$9,10,17,19,28,29,30,35,36,40$ 
Models (cont.)
Statistical-dynamical
Validation of

Moisture

Monsoons

Monte Carlo methods

Mount Pinatubo

Multifilter rotating shadowband radiometer

National Institute for Global

Environmental Change

Nephelometer

NIGEC

Nighttime temperature

Nighttime water-vapor profiles

Nitrogen cycling

Nucleation droplet

Numeric data packages

Nutrients

Ocean circulation

Oceanic uptake

Oceans

Thermal storage by
Ventilation of
Orange trees
Oscillations
Ozone
Depletion of
Pacific Ocean
Paleoclimate analysis
Parallel computers in
climate modeling

Parallelized codes
23,41

$2,3,6,7,15,17,19,20,21$, $29,30,31,44,62,66,73,99$, $105,107,108,116,121,122$

$1,14,15,28,30,34,39$, $47,64,82,89,90,95$, $105,108,111,115,116$ $7,17,19$ $65,67,138$ $19,20,54$ $54,76,85$ xii, xiv, 153-180 xii, xiv, 153-180 $9,11,71,131$ 74 ix, $78,95,101,102,103$, $108,109,110,112,113$, $114,116,117,133$ 56,68 150 $105,108,109,110,112,114$, $116,117,118,121,122$, $126,127,130,133$ $7,24,26,36,40,102$, $103,119,121,122,127$, $128,129,130,132,133,134$ 101,129 vi, $x, x i, x i i, x i v, 1,2,6,7,8$, $11,12,13,14,15,16,17,18$, $19,21,22,23,24,25,26,27$, $32,33,34,35,36,37,38,39$, $40,41,42,44,45,51,53,56$, $69,72,78,79,86,89,93,98$, $99,101,102,103,114,115$, 119-134, 137 52,130 111

17

$10,15-17,20,21,43,74,143$ 6,135

$45,86,89,101,121$, $125,126,128,130,131,132$ $11,15,17,19,41,52,122$

$$
2,21-40,42,43 \text {, }
$$

$49,67,79,80,88,102,132$ 28-30, 43
Parameterizations

Boundary-layer-depth

Partial pressure of $\mathrm{CO}_{2}$

Partial pressure of $\mathrm{N}_{2} \mathrm{O}$

PCMDI

$\mathrm{pCO}_{2}$

Peat,

Pore-water in

PEPCase

Pests

Phosphate

$x i, 6,10,14,15,20$, $22,24,28,31,36,37,38$, $46,49,58,61,63,68,69,70$, $71,73,78,79,82,84,90,91$, $103,107,120,131,138$

$99,122,127,128$, $129,130,131,132,133$ 133

$2,3,44$

$99,122,127,128$, $129,130,131,132,133$

114

57

113

Photometric studies of clouds

111,121

Photosynthesis

92

Phytoplankton,

iron-limited $\mathrm{CO}_{2}$ uptake by

PICL.

Planetary boundary layer

$97,98,105,107,108,109$, $111,114,115,116,117,118,127$

Plant communities

Plant competition

Plant growth

127

29,49

$5,6,7,22,30$, $34,38,63,82,91$ $57,108,109$

109

$97,98,105,107$,

Platforms, instrument

$110,111,115,116,118$ $\mathrm{xi}, 27,46,92$

Pleistocene, $\mathrm{CO}_{2}$ levels during the 118

Plume formation 123

$\mathrm{pN}_{2} \mathrm{O}$ 133

$\mathrm{PO}_{4}$ 130

Polar stratospheric clouds

Polarization-diversity lidar

Policy issues

80,85

$v$, ix, $x, x i i, 15,39$,

$105,135,139,140,141$,

$142,143,145,150$

Policy-makers

141

Population

Population dynamics

Postdoctoral education

$96,97,99,102,104,111,148$

Postdoctoral research program

Prairie ecosystems

PRC

Precipitation

PROBE

Productivity

Below-ground
111

115-117

$9,14,15,17,97,150$

$1,4-6,9-11,17,18,26,28,29$, $44,48,53,54,56,58,70,96,100$

$56,73,74,78,83,89$

$105,108,109,113,117$, $126,127,133,135,138,144$
115 


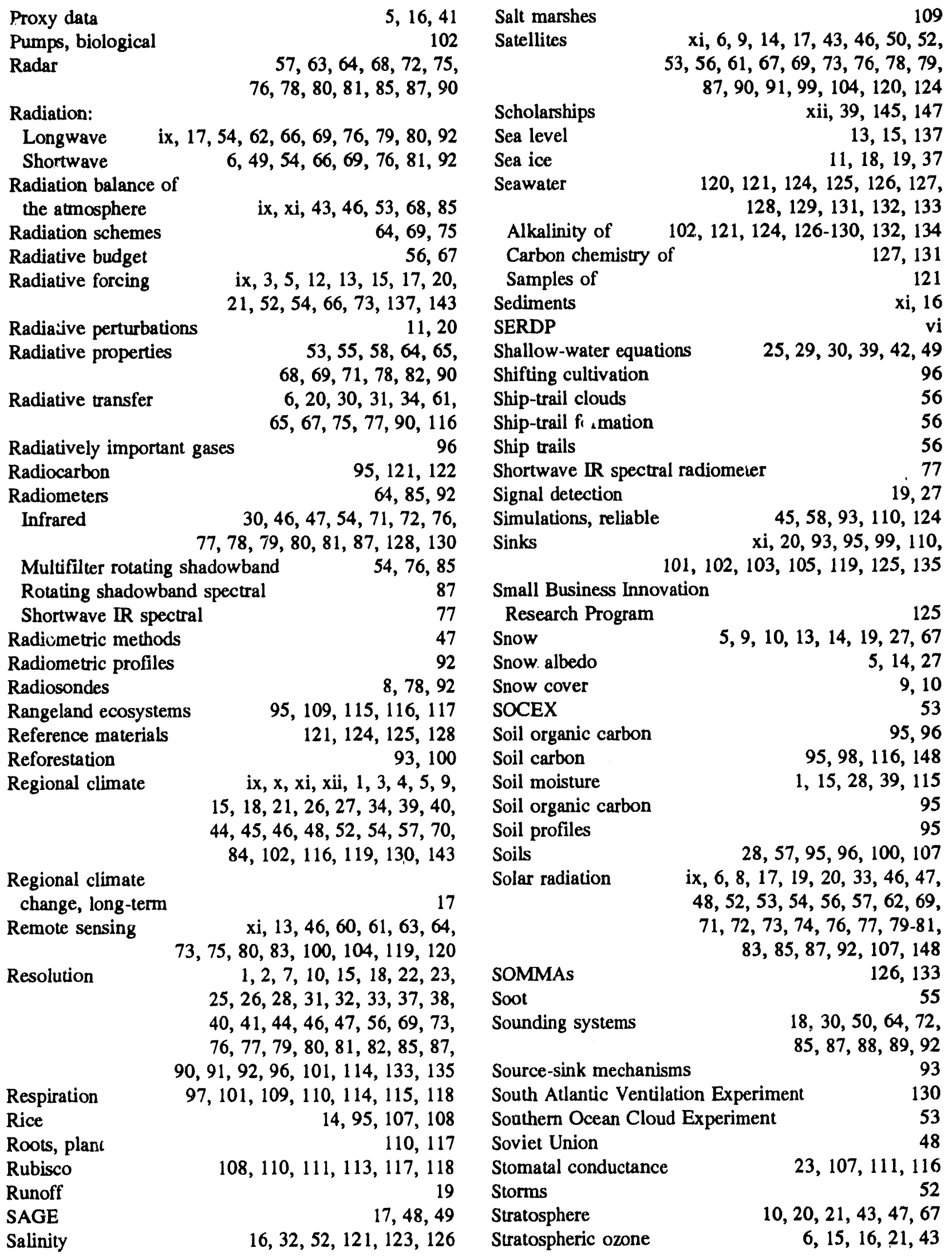




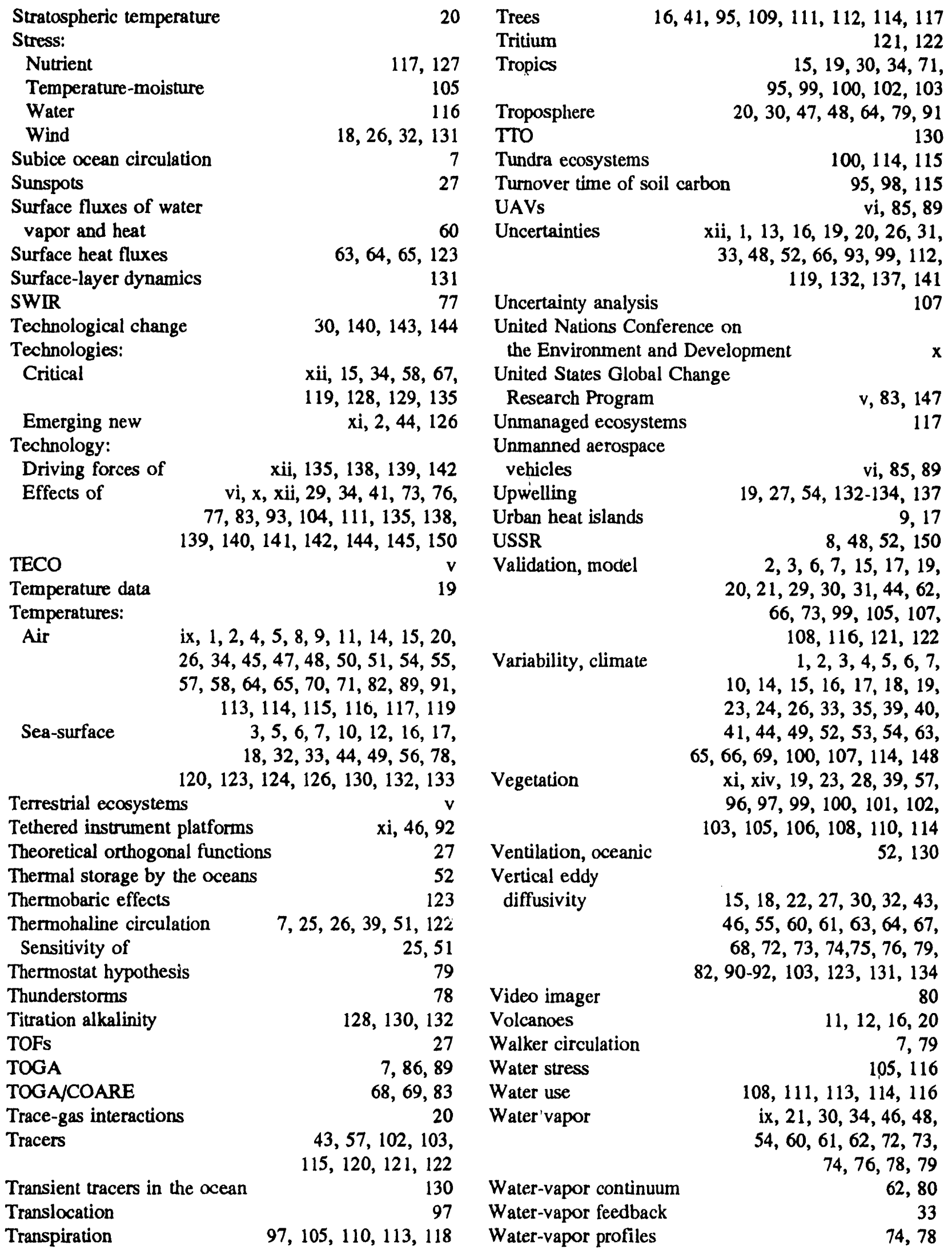


Waves

Weather, extreme

Weather patterns

Wetlands

Wheat

Whitecap-mediated gas exchange

Whole-sky imager

Wind stress
$5,20,30,32,34,131$

$1,9,70$

10,26

108,109

107,111

120

70

$18,26,32,131$
WOCE

Workshops

World Ocean Circulation

Experiment
$119,120,121,122,123,124,125$, $127,128,129,130,131,132,133,134$

$7,13,16,29,35,39,77$, $79,125,139,140,145,148$

$119,120,121,122,123$, $124,125,127,128,129$, $130,131,132,133,134$ 


\section{PRINCIPAL-INVESTIGATOR INDEX}

Ackerman, Thomas P.

Acock, Basil

Adams, John L.

Allen, L. H.

Anderzon, John $R$.

Anakawa, Akjo

Alher, W. E.

Avlasar, Ronl

Buder, David C.

Bacr, Ferdinand

Baerwald, Thomas J.

Barnes, Fairley

Ramett, Tim P.

Baumgardner, John R.

Buzzaz, F. A.

Bingler, Linda

Bjornstad, David J.

Boden, Thomas

Bradley, Raymond S.

Broecker, Wallace S.

Brown, Sandra

Byme, R. Nelson

Cess, Robert D.

Cessil, Paola

Chervin, Robert M.

Clough, Shepard A.

Couton, William B.

Coulter, Richard L.

Cox, Stephen K.

Crane, Robert G.

Crowley, T. J.

Cushman, Robert M.

Dabberd, Walter F.

Dannevik, William P.

Daum, Peter $\mathrm{H}$.

Del Genio, Anthony D.

Denbo, D. W.

Deroos, Bradley G.

Dickinson, Robert E.

Dickson, Andrew G.

Doran, J. Christopher
Dowlatabadi, $\mathrm{H}$.

Downing, John $P$.

125,126

Drake, Bert G.

Drake, John B.

Druyan, Leonard $M$.

Edgerton, Sylvia

Edmonds, James A.

Ellingson, Robert G.

Emanuel, Kerry A.

Emanuel, W. R.

Emerson, Steven

Entzminger, John N.

Falkowski, Paul G.

Farrell, Brian F.

Fischhoff, B.

Foster, I. T.

Fung, Inez Y.-S.

Gates, W. Lawrence

Gautier, Catherine

Ghan, Steven J.

Goldsmith, J.

Goyet, Catherine

Gray, $\mathrm{H}$.

Gunst, R.

Gutowski, William J.

Hack, James J.

Hall, Charles A. S.

Hameed, $S$.

Hanson, Howard P.

Harrison, Lee

Hellmer, H. H.

Hendrey, George

Heymsfield, Andrew J.

Hoffert, Martin I.

Hosker, R. P.

Houghton, R. A.

Hudson, James G.

Huntington, H. G.

Jacoby, Henry

Jacobs, S. S.

Jensen, $T$.
109

28,49

6

66

96,139

50,66

29

97

126

66

127

30

138

30

99

44

67

68

73

127

51

51

7,31

31

99

5

51

76

7

110 
Johnson, Donala' R.

Johnson, $\mathrm{K}$.

Jones, P. D.

Kao, C. Y. Jim

Karl, Thomas R.

Keeling, Charles D.

Kegelmeyer, W. Philip, Jr.

Key, R. M.

Khalil, M. A. K.

Kiehl, Jeffrey $T$.

Kimball, Bruce

King, A. W.

Kinter, James L.

Kleinman, L. I.

Kliewer, Kenneth L.

Ko, Malcolm K. W.

Kutzbach, J. E.

Kropfli, Robert A.

Kukla, George

Kulp. Thomas J.

Kunkel, $\mathrm{K}$.

Lacis, Andrew A.

Lambert, $\mathbf{R}$.

Lapp, $\mathbf{M}$.

Laulainen, Nels S.

Lave, L. B.

Leiby, Paul N.

Leifer, Robert

Leone, John, Jr.

Lincoln, David E.

Lindzen, Richard S.

Louis, Jean-Francois

Luxmoore, R. J.

MacCracken, Michael C.

MacKenzie, F. T.

Malone, Robert $C$.

Margolin, Len G.

Marotzke, J.

McCurdy, W.

McIntosh, Robert E.

Mechoso, C. R.

Meehl, G.

Meier, Mark F.

Melfi, S.

Michalsky, Joseph

Miller, A.

Millero, Frank J.

Mirin, A. A.

Mooney, Harold A.

Moore, B., III

Murcray, David G.

Nobel, Park S.
69,70

$8,9,10$

101,128

70

130

14

71

111

97

33

64

33

10

18

72

10

72

60

73

129

73

53

138

140

74

70

112

34

75

113

11,34

134

35

36

41

37

75

21

18

13

73

53, 76

24

129

27

112

103

77

113
Nakicenovic, Nebojsa

140

Norby, Richard J.

North, G. R.

113

North, Gerald R.

Novakov, Tihomir

Nowlin, W. D.

Oechel, Walter C.

Oglesby, R. J.

Ohring, George

O'Neill, E. G.

Owensby, Clenton E.

Palusykiewicz, Theresa

Peng, T.-H.

Penner, Joyce E.

Pfeffer, W. Tad

Piacsek, S. A.

Platt, C. M. R.

Porch, William B.

Post, W. M.

Preller, R. H.

Prinn, Ronald G.

Ramanathan, V.

Randall, David A.

Rasmussen, R. A.

Revercomb, $\mathrm{H}$.

Reynolds, James F.

Richards, John F.

Robock, Alan

Rouhi, Safiali

Sarmiento, Jorge L.

Sassen, Kenneth

Schimel, David

Schlesinger, Michael E.

Siegel, Donald I.

Semtner, Albert J.

Siegenthaler, U.

Simpson, M. L.

Skole, David J.

Skyllingstad, E. D.

Small, $\mathrm{H}$.

Smith, Richard D.

Smith, William L.

Somerville, Richard C. J.

South, D.

141

Spaeth, Warren T. $\quad 83$

Spinhirne, James $\quad 83$

Spratlin, T. L. $\quad 81$

Sprigg, William 16

Stamnes, Knut $\quad 58$

Stephens, Graeme L. 64

Stewart, H. Bruce 41

Stokes, Gerald M. $\quad 84$ 
Stone, Peter $\mathbf{H}$.

Strain, Boyd R.

Stull, Roland B.

Swisshelm, Julie

Takahashi, Taro

Toman, Michael A.

Toon, O. B.

Uhlir, Paul F.

Walcek, Chris J.

Wallace, Douglas W. R.

Wang, Wei-Chyung

Washington, Warren $\mathbf{M}$.

Webb, Thompson, III
Weiss, R. F. 133

Westphal, D. $\quad 90$

Weyant, J. P. 142

White, Andrew B. $\quad 42$

$\begin{array}{rll}131,132 & \text { Whiteman, Charles D. } & 92 \\ 142 & \text { Wigley, T. M. L. } & 19\end{array}$

90 Williams, Allen L. 58

151 Williams, Edward R. 143

90 Winn, C. D. 134

132 Wohlpart, A. $\quad 59,147$

$17,91 \quad$ Wódward, Wayne A. 51

18 Wuebbles, Donald J. 20, 42, 143

18 Yang, Zili 143

93
2
2
2
9
8
3
4
7
1
3
3




\section{INSTITUTION INDEX}

Advanced Research Projects Agency

Applied Research Corporation

Argonne National Laboratory

Atmospheric and Environmental

Research, Inc.

Atmospheric Turbulence and Diffusion

Division, NOAA

Battelle Marine Sciences

Laboratory

Battelle Memorial Institute

Brookhaven National

Laboratory

Brown University

Camegie-Mellon University

Colorado State

University

Columbia University

Committee on Earth and

Environmental Sciences

Commonwealth Scientific and

Industrial Research Organisation

Desert Research Institute

Duke University

Environmental Measurements Laboratory

Goddard Institute for Space

Studies

Harvard University

Illinois State Water Survey

Institute of Arctic and Alpine Research

Institute of Global Environment and

Society, Inc.

International Institute for Applied

Systems Analysis

Iowa State University

Kansas State University

Lamont-Doherty Earth

Observatory

Lawrence Berkeley Laboratory

Lawrence Livermore National

Laboratory

Los Alamos National

Laboratory
$120,123,125,126,131$

124

$41,64,110,127,132$

18

138

$13,32,38,47,63,64,79$

$6,7,10,95$

4

53

$102,116,117$

74

$48,73,99$

30,108

58

13

33

140

7,31

115

$121,131,132$

55

$11,20,27,34,37,42$, $44,56,72,143$

$25,35,36,40,42,56$, $60,69,70$
Massachusetts Institute

of Technol sgy

NASA Ames Research Center

$29,34,41,141$

90

NASA Goddard Spaceflight Center $\quad 83$

National Academy of Sciences

National Center for Atmospheric

Research

$18,26,31,64,68,71$

National Climatic Data Center

$8,9,10$

National Oceanic and Atmospheric

Administration

77

National Science Foundation

129

Naval Postgraduate School $\quad 40$

Naval Research Laboratory $\quad 38$

New York University 52

Oak Ridge Institute for Science

and Education

59,147

Oak Ridge National

Laboratory

$28,33,47,49,81,97$,

$113,122,129,137,140,150$

Office of Policy, Planning, and Program

Evaluation

143

Oregon Graduate Institute

Pacific Northwest

Laboratory

$23,37,53,65,68,83,84$,

$92,96,139$

Pennsylvania State University

26,60

Plant Stress and Protection Research Unit

108

Princeton University

102,130

Purdue University

18

Rand $\quad 137$

Resources for the Future $\quad 142$

Rutgers University 22

San Diego State University $\quad 114$

Sandia National

Laboratories $\quad 42,70,73$

Science Applications Internal onal

Corporation

61

Scripps Institution of

Oceanography

$24,78,82,101,124,128,133$

Smithsonian Institution

109

Southern Methodist University

51

Stanford University

State University of New York

112,142

at Albany

$17,53,76,90,91$ 
State University of New York at Stony Brook

State University of New York at Syracuse

Syracuse University

Systems Research Laboratory

Texas A\&M University

University Corporation for

Atmospheric Research

University of Alaska

University of Arizona

University of Bern

University of California at

Los Angeles

Univensity of California at San Diego

University of California at Santa Barbara

University of Colorado

University of Denver
University of East Anglia

19

5,61 University of Hawaii

University of Illinois

99 University of Maryland

57 University of Massachuseus

107 University of Miami

$15,60,95$

$15,23,50,66$

4,75

129

$\begin{array}{ll}\text { University of New Hampshire } & 103 \\ \text { University of South Carolina } & 112\end{array}$

39 University of Utah 80

58 University of Washington

28 University of Wisconsin

103 Water Conservation Laboratory

Wave Propagation Laboratory/

$18,21,32,79$

126

111

21,113 . NOAA/ERL

72

25, 39 Western Cotton Research Laboratory

111

67 Woods Hole Oceanographic

51 Institution

127

77 Woods Hole Research Center 

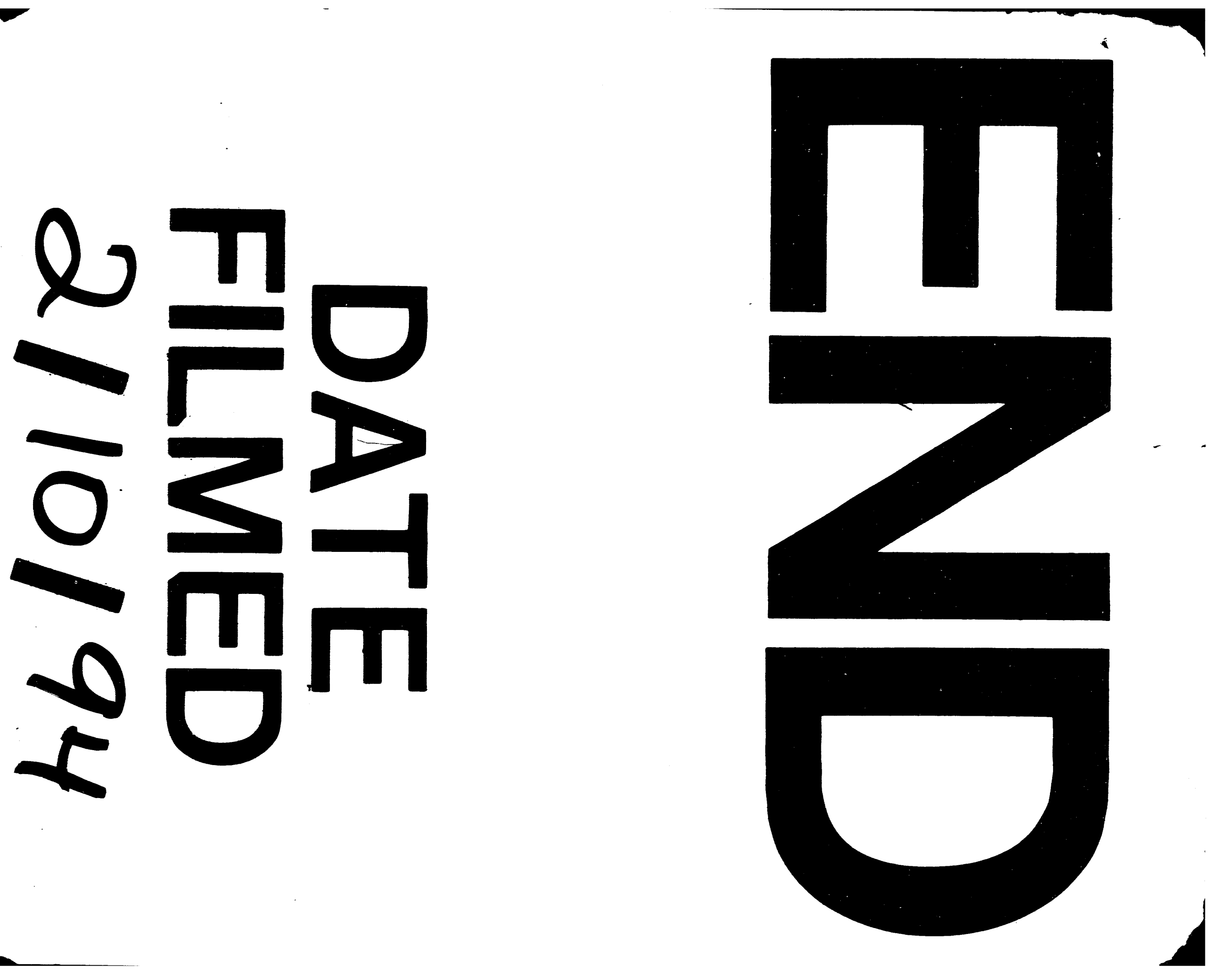
